This document has been digitised by the Bodleian Libraries, University of Oxford as part of the Oxford Digital Library for Forestry (ODLF) project.

Digitisation of this document has been made possible through the support of the Andrew W. Mellon Foundation.

The original contents of this document remain the copyright of the University of Oxford (http://www.ox.ac.uk/).

For enquiries please contact: enquiries.rsl@bodleian.ox.ac.uk 


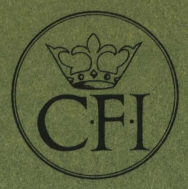

TROPICAL FORESTRY PAPERS. No. 10

\section{A MANUAL ON SPECIES AND PROVENANCE RESEARCH WITH PARTICULAR REFERENCE TO THE TROPICS}

Compiled by J. BURLEY and P.J. WOOD

DEPARTMENT OF FORESTRY

COMMONWEALTH FORESTRY INSTITUTE

UNIVERSITY OF OXFORD

1976 

A

M A N U A L O N

S P E C I E S A N D P R O V E N A N C E R E S E A R C H W I T H PAR T I C ULA R R E F E R E N C E

$\begin{array}{cccccc}T & 0 & T & H & E \\ T & R & 0 & P & C & S\end{array}$

Compiled by

J. BURLEY and P.J. WOOD

from contributions by

P.G. Adlard, I.A. Andrew,$\underline{\underline{1}}$ J. Burley, A. Greaves, J.F. Hughes $/ \underline{\underline{2}}$

R.H. Kemp, R. Lines, $\underline{3}$ R.A. Plumptre, B.T. Styles, R.L. Willan, $/ 4$ P.J. Wood, H.L. Wright

I 1 Formerly CFI, Oxford; now Forest Research Institute, Rotorua, New Zealand.

12 University Lecturer, Department of Forestry, University of Oxford.

$/ \frac{3}{4}$ Forestry Comnission, 231 Corstorphine Road, Edinburgh Scotland.

14 Forestry Department, FAO, Rome, Italy.

All others are members of the Unit of Tropical Silviculture, Commonwealth Forestry Institute, Oxford. 



\section{Preface}

Professor J.L. Harley, F.R.S.

This, number 10 in the Unit of Tropical Silviculture's renamed series "Tropical Forestry Papers", is the first contribution which is not a species monograph or bibliography. Although aimed primarily at tropical countries, and especially those with limited research experience or facilities, it attempts to provide a comprehensive treatment of the principles and practices involved in testing species and provenances that are applicable under all forest plantation conditions. It represents the work of nine members of the Unit, one member of the Department and two other specialists who between them have had experience in all phases of this type of work in both tropical and temperate regions.

This final version has been rewritten from an original draft which was submitted to the Tenth Commonwealth Forestry Conference, and it incorporates the suggestions and contributions of many leading specialists throughout the world. Clearly such a wide-ranging book cannot be a textbook for all the subjects included and for a number of subjects textbooks already exist, e.g. taxonomy, biochemistry and statistics. Nevertheless it is hoped that the book will present even these subjects in a rather different, practically orientated light, and that it will be a valuable contribution to the field forester's library. 


\section{TABLE OF CONTENTS}

Preface (J.L. Harley)

1. POLICY AND PLANNING

1.1 Background to the Manual

1.2 Policy, planning and objectives

1.21 Objectives and policies for production forestry in the tropics

1.211 The basic problems in production forestry

1.212 Objectives and policies of production forestry in the tropics

1.213 Planning and implementation of afforestation schemes

1.214 The need for research

1.215 International co-operation in tropical plantation forestry

1.22 Planning and organization of species and provenance trials in the tropics

1.221 Selection of objectives

1.222 Statement of policies

1.223 Overal1 plans

1.224 Research programmes and projects

Phasing of species and provenance trials

1.231 The need for phased trials

1.232 The types, sequence and time scale

1.233 The importance of seed

1.234 Control of trials and their follow-up

2. THE BASE POPULATION

2.1 Taxonomic and biosystematic studies

2.11 Variation, sources and types

2.12 Taxonomic characters

2.13 Classical taxonomic categories

2.131 Genus

2.132 The species

2.133 The subspecies

2.134 Variety

2.14 Nomenclature

2.15 The biological species

2.151 Implications of the breeding system 20

2.152 Problems of uniparental reproduction 20

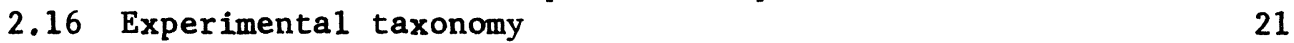

2.161 Ecospecies $\quad 22$

2.162 Ecotype 22

2.163 Clines 23

$\begin{array}{ll}2.164 \text { Deme } & 24\end{array}$

2.17 Provenance $\quad 24$

2.18 Cultivar 24

2.19 Modern methods of studying species 25

2.2 The application of biochemical methods 26

2.21 Introduction 26

2.22 Topics in which biochemical methods are applicable 27

2.221 Studies of inter-and intra-specific variation 27

2.222 Estimation of genetic variation within populations 
Page

2.223 Identification of genetic markers and indirect selection

2.224 Evaluation of biochemical products

2.225 Eeed source 29

$\begin{array}{ll}2.23 \text { Biochemical characters } & 29\end{array}$

$\begin{array}{ll}2.231 \text { Mineral nutrients } & 30\end{array}$

$\begin{array}{ll}2.232 \text { Organic compounds } & 30\end{array}$

2.24 Techniques 31

2.3 Seed procurement for species and provenance research 32

2.31 Objectives of seed procurement 33

2.311 Seed source trials (provenance trials) 33

2.312 Population improvement 33

2.313 Seed research $\quad 34$

2.314 Gene conservation $\quad 34$

2.32 Planning seed procurement 34

2.321 Survey of information on species and areas for
collection

2.322 Contacting officials and collaborators 35

2.323 Field reconnaissance 35

2.324 Timing of seed collection 36

2.325 Choice of tactics and methods for seed collection 36

2.326 Planning collection of correlated material and
data

2.327 Planning seed handling, transport and storage 37

2.328 Selection of staff 38

2.33 Organization of seed procurement 39

2.331 Delivery of staff and equipment 39

2.332 Exploration $\quad 39$

2.333 Selection of stands and trees 40

2.334 Selection of reproductive material 43

2.335 Techniques of collection 44

2.336 Field records $\quad 45$

2.337 Certification of origin 46

2.338 Temporary storage and transport $\quad 47$

3. SITE ASSESSMENT IN SPECIES AND PROVENANCE RESEARCH 49

3.1 Purpose of site assessment in species and provenance research 49

3.2 Proposed methods of site assessment 49

3.21 Assessment of the sites of seed sources 49

3.22 Assessment of proposed planting areas as an aid to
planning trials

$\begin{array}{ll}3.221 \text { Generalized procedure } & 49 \\ 3.222\end{array}$

3.222 The land system procedure 51

3.223 The distribution and location of trials 52

3.23 The assessment of existing plantation sites as an aid
to planning sample plot work

3.24 Application of the results of species and provenance
trials

3.3 Assessment of the influence of environmental factors on species and provenance growth 54

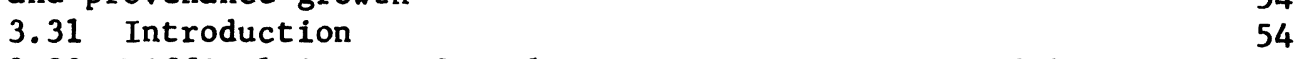

3.32 Difficulties in formulating site assessment models 55

3.33 Methodology 56

3.34 Description of the environment 57

3.341 Classification framework 57

$\begin{array}{ll}3.342 \text { Trial site description } & 57\end{array}$ 
3.4 Advanced descriptions of climatic conditions 61

3.41 Thornthwaite's method 61

3.42 Penman's method $\quad 62$

3.43 The water balance $\quad 62$

3.431 Computation of the water balance 63

3.5 Data analysis

3.432 Presentation of information on the water balance 65

4. PRINCIPLES OF EXPERIMENTAL DESIGN

$\begin{array}{lll}4.1 & \text { Sources of variation } & 67\end{array}$

$\begin{array}{lll}4.11 & \text { Scales of variation } & 68\end{array}$

4.12 Models of variation 68

4.2 Experimental design for species and provenance trials 69

4.21 Randomized complete block designs 71

4.22 Incomplete block designs 73

4.221 Lattice designs 73

4.23 Other types of design 76

4.231 Fully randomized design $\quad 76$

4.232 Non-orthogonal blocked designs $\quad 76$

4.233 Latin squares $\quad 76$

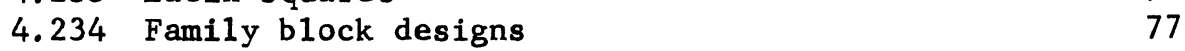

$\begin{array}{ll}4.235 \text { Systematic designs } & 79\end{array}$

$\begin{array}{lll}4.24 & \text { P1ot size and type } & 79\end{array}$

4.25 Layout and demarcation of the trial 80

4.26 Experimental design in the nursery 81

4.27 Series of experiments 81

4.28 Comparison of designs 81

5. A GUIDE TO FIELD PRACTICE $\quad 83$

5.1 Objectives and planning 83

5.11 General considerations $\quad 83$

5.12 Objectives $\quad 84$

5.13 Planning and control of trials 84

5.14 Practical 1imitations $\quad 85$

5.15 Phasing and time scales of species trials 86

5.151 The arboretum phase $\quad 86$

5.152 The species elimination phase $\quad 86$

5.153 The species testing phase $\quad 86$

5.154 The species proving phase 87

5.155 Phasing and time scales of provenance trials 87

5.156 Range-wide provenance phase 88

5.157 Restricted provenance phase $\quad 88$

5.158 Provenance proving phase $\quad 88$

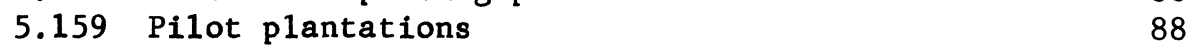

5.2 Selection of sites, species and provenances 89

5.21 Selection of sites and collection of site data 89

5.211 Stratification of sites $\quad 89$

5.212 The collection of site data 89

5.213 Climatic data $\quad 89$

$\begin{array}{lll}5.214 & \text { Soil types } & 90\end{array}$

5.215 Topography and location 90

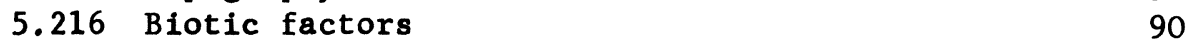

5.217 Vegetation 90

5.22 The selection of species and provenances 90 
5.221 Choice of product

Page

5.223 Seed 91

5.3 The nursery stage in trials 92

5.31 The location of nurseries 92

5.32 Experimental design in nurseries 92

$\begin{array}{ll}5.33 & \text { Nursery practice }\end{array}$

5.331 Germination 93

5.332 'Pricking out' or transplanting 93

5.333 Culling 93

5.334 Size of plants 93

5.335 Mycorrhiza 93

5.34 Priorities in nursery assessment 94

5.35 Early testing and controlled environments 94

5.351 Biosystematic studies $\quad 95$

$\begin{array}{ll}5.352 \text { Juvenile-mature correlation } & 95\end{array}$

5.353 Seed source identification 95

5.354 Studies in controlled environments 95

5.4 The field stage 95

5.41 Objects of experiments 95

5.411 Duration of trials $\quad 95$

5.412 Site selection and demarcation 96

5.42 Experimental design in the field 96

5.421 Plot size, shape and competition 97

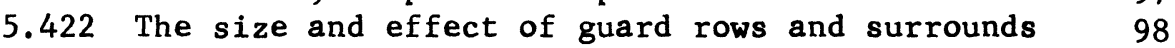

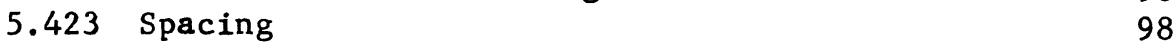

5.424 Repetition of experiments in time 99

5.43 Site preparation and fencing 99

$\begin{array}{lr}5.431 \text { Ground preparation } & 99\end{array}$

$\begin{array}{ll}5.432 \text { Fertilizing } & 100\end{array}$

$\begin{array}{ll}5.433 \text { Fencing } & 100\end{array}$

$\begin{array}{ll}5.44 \text { Pre-planting activities } & 100\end{array}$

5.441 Transport of planting stock to the field 100

5.442 Identification of planting spots and individual trees 101

$\begin{array}{ll}5.45 \text { Planting and replacements } & 101\end{array}$

$\begin{array}{ll}5.451 \text { Planting } & 101\end{array}$

$\begin{array}{ll}5.452 \text { Replacements } & 101\end{array}$

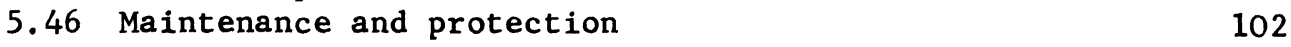

$\begin{array}{ll}5.461 \text { Weeding } & 102\end{array}$

$\begin{array}{ll}5.462 \text { Thinning } & 102\end{array}$

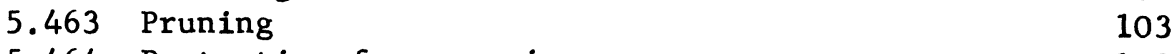

5.464 Protection from grazing 103

$\begin{array}{ll}5.465 \text { Fire } & 103\end{array}$

$\begin{array}{ll}5.47 \text { Priorities in field assessment } & 104\end{array}$

$\begin{array}{ll}5.48 \text { Recording systems } & 106\end{array}$

$\begin{array}{ll}5.5 \text { Use of results } & 107\end{array}$

6. EVALUATION 108

6.1 Assessment and analysis 108

$\begin{array}{lll}6.11 \text { Assessment } & 108\end{array}$

6.111 Seedling and nursery stage 108

$\begin{array}{ll}6.112 \text { Field stage } & 109\end{array}$

$\begin{array}{ll}6.12 \text { Data recording } & 110\end{array}$

6.121 Standard forms for recording data 111 
6.122 Other methods of recording data 111

$\begin{array}{ll}6.13 \text { Statistical analysis } & 112\end{array}$

6.131 Preliminary analysis 113

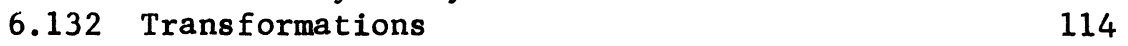

6.133 Analysis of variance 115

6.134 Comparison of means, tests of differences 121

6.135 Components of variance 123

6.136 Analysis of covariance 124

6.137 Correlation and regression 125

6.138 Series of experiments: genotype-environment

$\begin{array}{ll}6.139 \text { Multivariate analysis } & 129\end{array}$

6.2 Sample design for research in natural stands or
plantations

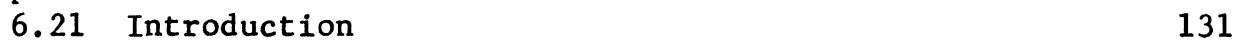

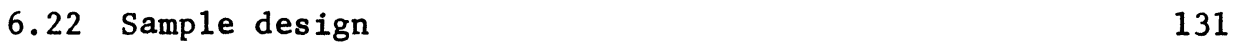

6.221 Simple random sampling 132

6.222 Stratified random sampling 134

6.223 Systematic sampling 136

6.224 Multi-stage sampling (sub-sampling) 137

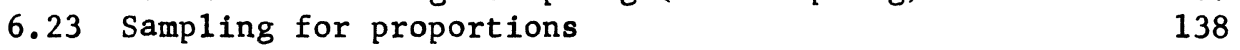

6.231 Simple random sampling for proportions 139

6.232 Cluster sampling for proportions 140

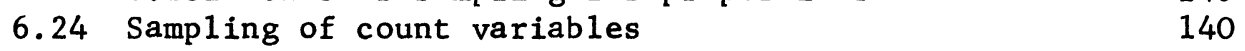

$\begin{array}{ll}6.25 \text { Classification data } & 140\end{array}$

6.26 Shape and size of sampling units 141

$\begin{array}{lll}6.261 \text { Shape } & 141\end{array}$

$\begin{array}{ll}6.262 \text { Size } & 141\end{array}$

$\begin{array}{ll}6.27 \text { Growth plots } & 141\end{array}$

6.3 Assessment of wood quality 143

6.31 A plan for the research required to investigate wood quality and the possibilities of substantial improvement in wood quality in afforestation schemes 143

6.32 The use of key index features as indicators of wood quality

6.33 Direct testing of wood properties and use characteristics

6.34 Suggested procedures for the evaluation of wood quality in relation to species and provenance research
6.341 Pilot tests
6.342 General utilization tests
6.343 Tests designed to study variation in properties, particularly in relation to the effects of genetic factors and of site con- ditions
6.344 Specialized tests 
Appendices

1. Glossary and symbols

1.1 Glossary of some terms used in the Manual

168

1.2 A selection of symbols for forest mensuration

2. Seed collection

2.1 Annotated 1 ist of equipment which may be needed for collection of seed, site information and herbarium specimens

2.2 Examples of seed collection data sheets

3. Site assessment methods

3.1 Example of a land system description (Astle et al., 1969)

3.2 Kuchler's (1949) scheme of vegetation description Vegetation description forms $1-3$

3.3 Soil profile description forms 1-4

3.4 Climatic data and estimation of potential evapotranspiration and the computation of water balances

4. A selection of methods for determining the number of replications required

5. Plans for lattices with $12,16,20,25,30$ and 36 treatments

6. Determination of sample size

7. Nursery assessment

7.1 Nursery sampling technique

7.2 Characters of use in the assessment of species and prov-

enance trials at the nursery stage. (The suggested sample sizes are based largely on experience with pines and could be modified for other genera especially hardwoods.)

8. A selection of record forms

8.1 A. Nursery record form

B. Nursery assessment form 203

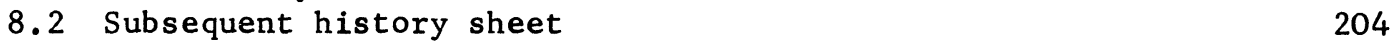

$\begin{array}{lll}8.3 \text { A. Periodic assessment form } & 205\end{array}$

B. Stem analysis form 206

$\begin{array}{ll}\text { C. Tree volume form } & 207\end{array}$

9. A selection of analytical formats modified from Dawkins (1968) 208

9.1 Analysis of variance: single classification (fully random) 209

9.2 Calculation of contrasts and their confidence limits by

Scheffe's method

9.3 Analysis of variance: simple randomised block 211

9.4 Analysis of variance: latin square (up to $6 \times 6$ ) 212

10. Sample plot procedure in commercial plantations 213

11. Spacing and stocking tables $\quad 224$

12. Outline of an experimental plan 226 
- (viii) -

Special appendices (obtainable separately from this Manual from CFI, Oxford)

1. Standard methods for recording information when collecting samples for wood quality tests.

2. Laboratory procedures for general utilization tests.

3. The wood structure index approach used at the Commonwealth Forestry Institute, Oxford, for the evaluation of wood quality. 
This Manual has been prepared at the invitation of the Food and Agriculture Organization of the United Nations (FAO) and the International Union of Forestry Research Organizations (IUFRO). It attempts to combine and expand two draft Manuals prepared by these organizations on the conduct of species and provenance trials respectively; for various reasons neither of these was formally published.

In 1962, Section 22 of IUFRO set up a "Working Group on Provenance Research and Testing" under the Chairmanship of Prof. Dr. Miroslav Vyskot; in May, 1963, he prepared a paper on "Standardisation of Methods for Provenance Research and Testing" which was published in the Proceedings of the World Consultation on Forest Genetics (Vyskot, 1963). Recommendation 8 (c) of this Consultation asked Section 22 of I.U.F.R.O. "to prepare instructions concerning the collection of seeds, the design of field experiments and evaluation of results to aid in the development of national and international studies of provenance". Prof. J.D. Matthews, the Leader of Section 22, and M. Pierre Bouvare1, Director of the Station d'Amelioration des Arbres Forestiers at Nancy, accordingly arranged a meeting of the Working Group, from September 6th-9th, 1965, at Pont-a-Mousson, France.

Using Prof. Vyskot's paper as a framework, more than sixty members of the Group discussed the subject under five main aspects: Kinds of Provenance Experiments and their Planning; Selection of Parent Stands and Procurement of Seed; Design and Layout of Experiments and Analysis of Results; Techniques and Assessments in the Nursery Stages, including Early Test Methods; and Techniques and Assessments in the Forest Stages. Draft chapters on each aspect were presented to the Working Group, discussed in full session, and edited by small committees. These edited chapters constitute the Report of the Working Group as compiled and edited by R. Lines of the United Kingdom Forestry Commission (Lines, 1967).

With the encouragement and financial support of the United Nations Development Program, New York, a small meeting of FAO/UNDP field officers was held in Trinidad from 4-9 December, 1967, on the occasion of the tenth session of the Latin American Forestry Commission. The opportunity was taken to invite several national forest officers working in the field of tree species trials to participate in the meeting. Some 12 men attended and considered it highly desirable to coordinate and, as far as possible, to standardize the methods for tree species trials in tropical America. Based on the reports of three drafting committees, the group produced a draft "Guide to tree species trials in tropical America" (FAO, 1968).

Many of the considerations of species and provenance testing are common, to both; further many countries, particularly in the tropics, are at an early stage of tree introduction. It seemed logical, therefore, to combine 
the earlier drafts (that were concerned mainly with the detailed management of field experiments) and to expand them to include sections on many other aspects of species and provenance research.

The Commonwealth Forestry Institute, Oxford,(CFI) agreed to undertake this task because its staff were closely concerned with international provenance testing of tropical species and because of its close links with FAO and IUFRO. A manual of procedures for the conduct of a joint FAO/FRI, Australia/CFI, Oxford, international provenance trial (of Pinus kesiya Royle ex Gordon) had already been prepared (Burley and Turnbull, 1970). Several CFI staff are also active in IUFRO as Deputy Divisional Coordinator, and Working Party Chairmen or Deputy Chairmen.

The CFI Unit of Tropical Silviculture is financed by contributions from most Commonwealth Governments. The programme is discussed every 5-6 years at the meetings of the Commonwealth Forestry Conference; indeed the concentration on fast growing tropical species, especially Central American pines, originated from resolutions of the eighth and ninth conferences in 1962 (Kenya) and 1968 (India) and was supported strongly at the tenth conference in 1974 (U.K.). Financial and moral support for the programe are given by FAO and IUFRO.

The CFI activities include exploration, conservation and utilization of forest genetic resources, together with advice and assistance to many countries (not only members of the Commonwealth) on all aspects of their programmes of tree introduction, management, improvement and evaluation. Staff of the Unit also assist with teaching and supervision of students. A11 of the staff have worked in the tropics and most continue to travel frequently in tropical and developing countries. This continual updating of awareness of current problems has allowed them to cooperate in the production of a Manual that should act as a checklist of considerations for the conduct of species and provenance research under a wide range of social and environmental conditions.

In the evaluation of a species, some activities may be undertaken by agencies other than the ones planting field trials, e.g. seed collection, taxonomy, evaluation of biochemical or wood properties; nevertheless an outline of those activities is given so that the field researcher is aware of the scope of complete "biosystematic studies", the term that is often considered analagous to "experimental taxonomy" and "provenance research". Species and provenance trials may be used for purposes other than those for which they were originally intended, e.g. studies of population genetics; it is not possible in a practical Manual of this type to include details of all such possible activities. Many of the topics discussed are specialized or controversial, e.g. site assessment and classification or wood quality evaluation; nevertheless it was felt desirable to introduce them in this Manual and, if the coverage could not be complete, then at least to provide references to major relevant publications so that the reader can pursue them for himself.

Throughout the Manual, wherever possible, priorities are indicated if a choice of action is possible; this is particularly important in 
relation to assessment of experiments. It is possible to measure many characteristics of nursery and field survival, growth, morphology, chemistry and anatomy of trees in a provenance trial; nevertheless not all of these could or should be assessed in each country. In internationally coordinated trials it is often possible for the coordinating agency to assess some traits centrally on behalf of all participants, $\underline{e} \cdot \underline{g}$. seed characteristics, or for one specialised laboratory to undertake studies

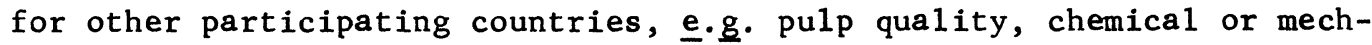
anical characteristics.

It is probably a sweeping generalization to state that, to date, the larger part of comparative species and provenance testing has been carried out by countries using exotic species. It is true that post-war trends in North America and Europe towards plantation forestry have stimulated greater efforts in provenance research but commonly even this research has been concentrated on species that are indigenous to the country or local area. Only comparatively recently has attention been given to provenance studies on exotic species, particularly through internationally coordinated, comparative trials. Certainly with the recent dramatic increase of plantations in the tropics, exotic species figure highly in species and provenance research in terms of numbers of species, numbers of countries and numbers of individual experiments.

There are obvious and subtle differences in both the theory and practice of provenance research between indigenous and exotic species; the questions asked, the priorities given (to type of testing and to characters assessed) and the use of the results will differ depending on the background knowledge of the species and its evolutionary history, including the man-made evolution of populations (see $\underline{\mathrm{e}} \cdot \mathrm{g}$. Jones and Burley, 1973). Nevertheless, it is hoped that this Manual will provide guidelines for the planning, conduct and use of species and provenance trials with both indigenous and exotic species. Although it has been prepared with the needs of tropical countries particularly in mind, it may prove useful for research in temperate regions. Throughout, the empirical rather than the academic approach is emphasized on the understanding that the primary aim of most afforestation programmes is the growing of healthy, productive forest. 


\subsection{Objectives and policies for production forestry in the tropics}

\subsection{The basic problems in production forestry}

Medium and long term forecasts, made by the Food and Agriculture Organization of the United Nations, indicate that very considerable increases in the consumption of wood can be expected in many tropical countries. Towards the end of the century, the annual world demand may be double that of the period 1960-2 (which was estimated then at 1,000 million cubic metres of industrial wood). It seems likely that there will be difficulties in meeting project requirements, even including those of some countries that have extensive areas of natural forests.

In parts of the tropics where natural forests are abundant, considerable proportions of these forests are often on land suitable for agriculture, and are therefore of uncertain value in planning for the forestry sector, because they are likely to be expropriated. Even where there are good prospects for long term management of natural forests, it may not be possible, because of the long term nature of natural forestry operations, to achieve high productivity before the end of the century.

Even where accessible, tropical forests often have a low stocking of valuable trees, and the greater part of the crop consists of less desirable or of unusable species. It would be very inadvisable to assume that it is only a question of time before a changing pattern of use, and improvements in technology, will lead to a solution of the problems of utilizing the greater part of the so called "secondary" and "weed" species. There are, in fact, usually very good technical reasons for classifying a wood as secondary. It is unlikely, for example, that such basic disabilities as poor tree form, marked difficulty in sawing, excessive distortion in drying, high resistance to nailing or adverse woodworking properties are likely to be overcome, to an extent or at a cost that will make it possible to promote the general use of these species for construction and for joinery timbers, although locally they may have some importance.

The trend towards increasing use of wood in the form of pulp or particles is also unlikely to make the management of natural forests more attractive. It may, in fact, have the reverse effect. Paper and board industries, to operate economically, require regular supplies of raw material of uniform size and quality. It is very difficult to meet these requirements, with the wide range of species and size classes commonly found in natural tropical forests. To avoid the need for importing pulp for blending, most existing or projected pulp schemes in the tropics are based on the use of exotic plantation species, either from the onset, or after a period during which mixed species from natural forests are accepted, at a calculated lower level of profitability, so that forest land can be cleared for planting.

Furthermore, the importance of wood for pulping relative to other 
purposes should not be forgotten. Whilst the demand for paper will, undoubtedly, increase in developing countries, a very much large amount of wood will be required in the form of sawn timbers. For the foreseeable future, the principal uses for wood (largely for fuel and for building) are facts that must be taken into account in selecting objectives and framing policies for forestry. The expenditure of valuable foreign exchange on importing forest products can be reduced by increasing home production from the natural forest through improved silviculture, or the establishment of plantations, or both.

An unfortunate factor in the management of natural tropical forest is the common and widespread problem of achieving a worthwhile increase in output and productivity in a few decades, or even in a full rotation. The growth response of trees of medium girth to exploitation felling is often disappointing, usually too little is known about the possibilities of enrichment planting to attempt this on a large scale, and in the longer term natural regeneration of the most desirable species is often sparse and some of these species are slow growing. The indications are that even from the better tropical high forests sustained yields are likely to remain low - that is of the order of $2-4 \mathrm{~m}^{3}$ per hectare per annum.

Where the yield per unit area of land is important, and where forecasts indicate that wood is likely to be in short supply 20-40 years ahead, there is an obvious incentive to establish plantations of trees. In the tropics, sustained yields of 15-20 cubic metres per hectare per annum ( $\left.\mathrm{m}^{3} / \mathrm{ha} / \mathrm{ann}\right)$ have been obtained with forest plantations on sites of reasonable quality (with the correct choice of species) and on better sites yields as high as $35 \mathrm{~m}^{3} / \mathrm{ha} / \mathrm{ann}$ are not uncommon. Much information is now available on the planting of both indigenous and exotic species in the tropics; however, there are many factors, in addition to rate of growth, that have to be considered in assessment of the feasibility of large scale afforestation schemes if maximum benefits and optimum economic returns are to be achieved when such projects are undertaken.

The possibility of reduced yields from plantations in second and later rotations in the tropics should not be overlooked but this need not be a serious risk provided that the proper silvicultural measures are taken, such as fertilization and the avoidance of soil compaction during harvesting.

One purpose of this Manual is to indicate how information can best be obtained for appraisal of some of the important technical and related economic considerations that have to be taken into account, in both the planning and the implementation of large scale plantation schemes. The political and social considerations that are of basic importance in planning for forestry, or on any other sector, are of course outside the scope of this Manual. Of course, forest production is not confined to wood or even to plant production. The natural forest particularly has a large role to play in animal production (grazing, wildlife) in recreation, in the protection of the soil, and in the production of regular supplies of water. 


\subsection{2 objectives and policies of production forestry in the tropics}

It is the duty of the forest administration to examine the possibilities for forestry in relation to national development as a whole. Foresters should put forward proposals, so that Governments can determine their objectives in the forestry sector and then formulate policies to achieve them. These proposals must, as far as possible, be soundly based on rational research programmes and on consideration of all likely alternatives to permit a certain degree of flexibility at this level. This is particularly important where classifications or maps of present land use and future land capability have already been compiled or where the national forest lands are insufficiently protected.

In many countries, the roles of natural and man-made forests should be considered as complementary, with natural forests fulfilling essential protective and cultural functions and providing certain special categories of wood, such as high quality material for cabinet making or veneering, while plantations supply an increasing proportion of requirements for constructional timber, utility sawn wood, wood based panels and pulp.

The basic, grand objective of production forestry is typically the provision of an improved national or regional supply of raw materials. In most countries the pressure on land is becoming steadily greater and, if the requisite raw materials can be produced from a much smaller area or in a shorter period by using intensive high-yielding plantation methods, foresters have a responsibility to employ these methods. Concentrated plantations lead to indirect economic and social benefits through the development of forest industries and opportunities for employment. Accordingly, plantations are being established on an increasing scale throughout the world. Plantation techniques and prescriptions can be more readily codified and implemented than those generally developed for the treatment of indigenous forests. This is frequently an important factor in countries where experienced staff are deficient.

Two factors are of primary importance in determining the role of afforestation in forest development, and in the use of resources, mainly of land and finance. First of al1, high productivity from plantation forestry will usually involve the use of sites of reasonable quality, so that there is likely to be competition with agriculture, particularly for land of favourable topography and fertility. The cost benefit analyses that are required for appraisal of plantation forestry projects in such competitive situations must be based on correct technical assumptions throughout all stages of afforestation, management, harvesting, conversion and marketing. Secondly, plantation forestry also requires a much greater initial expenditure, and subsequent costs for maintenance are also often higher than for improvement of natural forests, so that failure to make the correct choice of species and site may have very costly consequences. The possibilities of combining plantation establishment with agriculture (agrisilviculture, taungya) should not be overlooked. 
1.213 Planning and implementation of afforestation schemes

To meet national objectives for qualities and quantities of forest products a senior forestry executive, planner or policy-maker requires information from his silvicultural and research adviser. Clear answers require clear questions. The policy-maker cannot expect precise information unless he states precisely the objectives to be achieved.

However, there is no objection to, and often good reasons for, specifying more than one objective. In view of the long-term nature of forest crops and, in comparison, the speed with which technological preferences and markets can change, there is often a strong case for maintaining the maximum flexibility in the objectives. Flexibility is especially important with rotations longer than 15 years. If more than one objective is specified it is important to assign relative priorities. Once the objectives of the entire afforestation programme are defined the silvicultural considerations may be reduced to the interactions of three sets of factors, site, species and cultural techniques.

The correct choice of species and sites is the first stage in successful afforestation, but it needs to be followed by appropriate silviculture and management if optimum returns are to be obtained. The initial spacing and subsequent thinning, pruning, fertilization and other forms of silvicultural treatment, must be based on sound technical assumptions with regard to their effects on growth and on wood quality. More details of these factors are given in Chapter 5.

\subsection{The need for research}

For successful promotion of large scale afforestation projects in the tropics, there is need for carefully planned and well directed research, first of all to guide the selection of objectives and the formulation of policies, and then to support planning and the implementation of plans.

Species and provenance trials provide some of the basic information on which policies concerning afforestation are made, and there are obvious advantages if such trials can be initiated well in advance of the time when important investment decisions have to be taken. Many examples of failure, or of partial failure, of afforestation schemes, are recorded where preliminary trials were inadequate. While considerable progress has been made in the assessment of sites for planting by evaluation of geographic, climatic and edaphic factors, there are still serious limitations to the practical application of such techniques to select the species and provenance that are most suitable for the particular conditions (see Chapter 3 ). There is no substitute, at present, for adequate species and provenance trials on typical sites as a basis for large scale afforestation.

\subsection{International co-operation in tropical plantation forestry}

The considerations that must be taken into account in the promotion of large scale afforestation are similar in most tropical countries. 
There could be considerable benefits if different countries took a similar approach to planning for afforestation; it could reduce both the cost and the length of time required to obtain essential, basic, technical information. In particular, the standardization of procedures for experimental work would facilitate the exchange of information and the organization of international co-operation to promote research on afforestation. Species and provenance trials are an aspect of research in which international agreement and collaboration are of vital importance to all participants. A primary purpose of this manual is to put forward proposals for species and provenance trials on which such international co-operation could be based. Formal international projects attract more interest and resources when based on a well argued case with clear objectives.

1.22 Planning and organization of species and provenance trials in the tropics

1.221 Selection of objectives

Species and provenance trials should be conducted according to written plans, which will usually form part of the research programme of the national forest authority. Plans of this sort must state clearly the precise objectives of the particular species or provenance trial project. These should be derived from the objectives for national afforestation schemes, and must therefore take into account the priorities of such schemes and the constraints upon them. Achievement of the maximum benefits that can now be derived from research into optimum choice of species and provenance will depend to a very great extent upon realistic and constructive definition of the purposes of these investigations.

It is recognised, that for many of the trials carried out to date, objectives were not defined in the precise way suggested here. The results of some of these trials have undoubtedly provided very useful information for the promotion of afforestation, but it is also apparent that much was lost because the plans for these experiments were based on an inadequate assessment of their potential usefulness. It should also be admitted that research workers may frequently find it difficult to obtain guidance for the planning and management of species and provenance trials, when the objectives and policies for national afforestation projects are not adequately determined and stated. In such situations, however, if species and provenance trials are undertaken, a precise definition of the objectives of such research is still required, based if necessary on assumptions of likely alternatives for national objectives for afforestation. It may be necessary to change the objectives for research when national forest policies are finally determined, but no research project should be initiated without clear definition of its particular purposes.

The definition of objectives for species and provenance research should also include a clear statement of priorities, based upon the immediate, short term and long term requirements of the relevant afforestation programme. Constraints on the achievement of objectives also must 
be taken into account at the time of definition, and should indicate the limits of the resources (staff, facilities and finance) within which the project will be required to operate.

\subsection{Statement of policies}

The selection of objectives for species and provenance research, and the definition of priorities and constraints on these objectives, must be further qualified by statements of the policies by which it is hoped to achieve them. A statement of policies is the essential link between decisions on the objectives of a project and the planning that is required to attain such goals. For species and provenance trials policies should be concerned largely with the mobilization and allocation of resources (staff, facilities and finance) in relation to the agreed objectives. They need to take into account resources that are available locally, to recognise the distinction between forest services that have good research units and those that lack such facilities and to consider the availability of advice and assistance from other countries.

The stage of policy formulation in the development of species and provenance research provides the greatest opportunity for obtaining effective international co-operation. This can be achieved best by adopting a similar approach to such projects, as outlined here, $\underline{i} . \underline{e}$. by defining objectives, proceeding then to the formulation of policies, and eventually to preparation of detailed plans. It is suggested that an exchange of information on objectives and on available resources at the policy making stage would greatly increase the effectiveness of subsequent planning of both national and international trials. When plans have been made or put into operation it is often very difficult to amend them effectively, even when it is apparent that considerable benefits could follow from changes.

Departments that have well established silvicultural research units will be concerned largely with policy decisions concerning the emphasis to be placed on species and provenance trials in relation to other projects in their own research programmes and also with the extent to which they should collaborate in joint projects with other countries or in projects organized on an international basis. In countries that so far have not developed good research facilities more attention is required in policy making at this stage to obtaining the maximum possible benefit by the considerable advice and assistance that are available from other countries in this aspect of forest research. However, in both cases it cannot be overstressed that the way in which species and provenance trials are to operate must be stated clearly, if only in broad terms, so the research workers who are concerned with the implementation of policies have a proper basis for the next stage of planning of these complex projects.

\subsection{Overa11 plans}

In species and provenance research, planning is required at several levels, ranging from a plan for the overall control of the investigation in relation to objectives and policies, to detailed methods and procedures for specific experiments. 
The overall plan should proceed, from the definition of objectives and the broad statement of policies that will be pursued to achieve them, to deal with:-

(a) the selection, grouping, phasing and design of research projects leading to the preparation of appropriate programmes of work

(b) the operational requirements for research units to put these into effect.

It is suggested that more detailed plans for specific experiments in projects, and for services such as experimental design, computing and analysis should be in the form of appendices to the project plan. This will increase the usefulness of the plan as an operational document and will facilitate the addition of new research and the updating or amendment of existing projects and services.

\subsection{Research programmes and projects}

Research programmes will differ considerably depending on the requirements of particular countries but it would be of considerable advantage, in organizing or benefitting from co-operation between countries, if research programmes are compiled from consideration of an agreed standard list of the main activities in this sphere of research. It would be a further advantage if, for individual projects, the detailed procedures could also be agreed and standardized in so far as this is possible. (See Chapter 5 of this Manual). 
Research activities (suggested standard 1ist)

It is suggested that main activities in species and provenance research, from which programmes will be formulated, are as follows:-

(a) Review of literature, correspondence and personal knowledge of distribution and variation of species likely to be of value. Discussions with international and regional programmes concerned with these species.

(b) Choice of species and of provenances to test

(i) selection of parent stands in natural forest where possible based primarily on their seed production and genetic quality

(ii) selection and management of seed stands in plantations where possible

(iii) procurement, treatment and storage of seed

(c) Design, layout and analysis of species and provenance experiments

(i) assessment and selection of sites

(ii) selection of systems of silviculture and management

(iii) planning and design of experiments and interpretation

(iv) analysis and interpretation of experimental data

(v) report and dissemination of results

(d) Techniques and assessments in the nursery stage, including early test methods

(i) nursery conditions and cultural treatments

(ii) design and conduct of nursery experiments

(iii) nursery assessments

(iv) early test methods including biochemical and anatomical studies, juvenile-mature correlation

(e) Techniques and assessments in the forest stage

(i) definition of types of trials (short, medium and long term) and their objectives (see Chapter 5)

(ii) design and conduct of forest stage experiments (see Chapters 4,5 and 6 ).

(iii) assessments in the forest stage, including

1. survival

2. form and yield of individual trees and plots

3. wood quality, the factors that influence it, and their implications for utilization

1.23 Phasing of species and provenance trials

1.231 The need for phased trials

As has been repeatedly stressed above, more often than not adequate 
information is lacking, either on the requirements of the species, or on the characteristics of the site, or both. In such cases embarking on afforestation schemes without a carefully planned and executed experimental programme has often led to costly failures.

The choice of species to use for afforestation involves the extrapolation of information from elsewhere. Climatic and ecological matching of a new site and the original habitat of a species is rarely enough since it cannot reveal the adaptability of the species to new conditions or its ability to grow satisfactorily on a range of sites. When information is lacking, the best way to acquire it is through trials of a number of species in small plots on representative locations within the area of the proposed afforestation project. Provided the locations are carefully selected to sample the range of planting sites and are properly looked after, extrapolation of performance from small plots to the whole afforestation area should involve far less risk than imprecise comparisons, based on inadequate data, between widely separated regions of the world.

The advisability of species trials is now generally accepted, but the need for their careful planning and for high standards of maintenance and assessment has often been less appreciated. Species trials themselves can be wasteful and misleading if badly planned or executed and proliferation of plots, if they are ill-sited, ill-tended and ill-protected, is no substitute for a small, wisely planned programme which is tailored to the staff and financial resources available. The objective is to derive the greatest possible information from a given cost or, put the other way, to obtain the desired information at the lowest possible cost.

For species with naturally wide geographical or ecological ranges, provenance testing is essential. It is easy to be misled in the comparison of species for afforestation if the total range of intra-specific variation is not known.

Identification and comparison of sites must be done on an ecological, not a national, basis. Results from species trials in other countries but in the same climatic zone and on similar soils may be of closer application than those from trials on completely different sites within the same country. In order to make results in one country readily interpretable in another, the value of standardizing methodology, design, methods of assessment and recording, etc., cannot be over-emphasised. The remaining chapters of this Manual are intended to facilitate this standardization.

If detailed information is available on all factors significant to the success of a species in plantation, and on the status of these factors at the site to be afforested, it may be possible to start afforestation without preliminary species or provenance trials. More commonly, however, information has to be acquired gradually and, ideally, when starting from scratch, species and provenance trials should be phased according to the successions: - 
Little data on species Little data on sites Many species

Many provenances

Tested by small plots for short periods
Increasing data on species and site characteristics Fewer species

Fewer provenances

Tested by increasing plot size for longer periods

\subsection{The types, sequence and time scale}

The ultimate phase is, of course, the complete afforestation project where the source populations are reduced to one or two provenances of one or a few species and where the annual planting area is reckoned in hundreds or thousands of hectares. It must be recognized that there is no standard procedure or time schedule for passage through successive stages of testing; nor is there always a need to use every stage. However, the following distinct phases are commonly encountered and will usually be required either singly (sequentially) or in combination (telescoped) or at the same time (paralle1).

The species elimination phase is the mass screening of a large number of possible species in smal1 plots for a short period ( $1 / 10-1 / 5$ rotation) to determine survival and promise of reasonable growth. The species testing phase is assigned for the critical testing or comparison of a reduced number of promising species in larger plots for longer periods $\left(\frac{1}{4}-\frac{1}{2}\right.$ rotation). The species proving phase is designed to confirm, under normal plantation conditions, the superiority of a few probable species. Three similar stages apply to provenance testing for species with a wide natural distribution, a range-wide provenance sampling phase, a restricted provenance sampling phase and a provenance proving phase. Since these are generally applied to species that are promising or probable their plot size and duration can be larger than the comparable phases of species testing.

\subsection{The importance of seed}

For all stages of species and provenance testing, seed supply is a critical factor. Seed should be of authenticated origin. If large quantities might be required, then seed availability may become an overriding factor in species and provenance selection. If a particular species or provenance has outstanding qualities but cannot be planted extensively for lack of seed (e.g. Araucaria species), it is worth considering the establishment of limited areas of plantation as a local source of seed which can be used later to supply requirements for afforestation. The early establishment of seed stands, selection plantings and genetically superior seed orchards is strongly recommended to ensure a constant, local supply of seed and gene pool conservation. Consideration must also be given to seed storage. Large plantation programmes require large quantities of seed which must be carefully treated and stored to preserve viability.

With the increasing exchange of seeds and living plants there is an 
increased risk for the spread of pests and diseases. It is of utmost importance to develop methods of rating the hazard of potential pathogens, and to maintain facilities for prompt control action and quarantine regulations, based on the most recent knowledge.

\subsection{Control of trials and their follow-up}

The detailed planning and conduct of species and provenance trials is the responsibility of the silviculturist or research officer (see Chapter 5). However, the head of the forest service, or the policy-maker to whom the silviculturist is responsible must be capable of evaluating the research, and of deciding whether it will answer the right questions, with efficiency, and within the resources available.

Many heads of forest services have appointed Committees to review the programmes, procedures and progress of their research division, to indicate priorities among research projects, and to approve proposals for closing projects.

To facilitate this control the research staff must be required to produce and follow a project control plan for each species and provenance trial. This plan must be checked and approved by the head or the Committee before the project is initiated. After any amendments are made the head of the forest service should signify his approval in writing; this imposes on him the moral obligation to do his best to ensure continued finance and staff for the project. If there is no promise of continuity in this respect, there is little point in starting the programme. Once a project is initiated, however, regular reports should be required (usually annually).

A pilot planting project may frequently form an essential intermediate step between species/provenance trials and large scale afforestation. It will enable the forester to determine optimum cultural and managerial techniques and to make the vital decision on whether or not to proceed with the complete afforestation programme.

It must be remembered that in some cases the results of species and provenance trials are applied to the second, rather than the first, rotation. For example, many countries are already planting large areas of Pinus caribaea var. hondurensis Barr. \& Golf., mainly derived from the Mountain Pine Ridge, Belize. Indeed some countries have initiated selective breeding programmes based on this material. Nevertheless other provenances may be better or contain some valuable genes, and these countries should carry out comparative trials to identify such provenances for later planting. There is a danger that foresters who have a readily accessible seed source of a "satisfactory" species or provenance may feel it unnecessary to test others that could be potentially better. 


\subsection{Variation, sources and types}

Classification is basically an information storage and retrieval system and without it chaos would exist. Classifying is the basic process which man employs to deal with the vast array of diversity and enormous range of variation among individuals which he encounters in the natural world. He divides up this diversity into more and more manageable groups to which he then applies names. Classification and nomenclature are therefore the basis of communication. Different kinds of classification are made depending on needs but the most commonly used are based on as much data as possible and are multipurpose.

Variation makes classification both necessary and possible. Over a century ago Charles Darwin (1859) noted the universality of natural variation and this formed the inspiration for his great work on the evolution of species by natural selection. Basically three types of variation may be distinguished, all of which may result in differences between individuals. Firstly there is a component which is fixed and heritable and which is genetic; secondly there is an environmentally induced, non heritable component, and thirdly there is a part which we might call developmental. Juvenile foliage of many trees including conifers is very different from that of mature or adult trees. Leaves of epicormic shoots are generally larger than those from branches in the crown. In making classifications taxonomists have to be aware of those characteristics of the phenotype which are plastic and easily modified by the environment (such as leaf size and shape) and those which are more constant e.g. reproductive structures. Some of the underlying causes of genotypic variation are now well known (polyploidy, aneuploidy, etc.)

\subsection{Taxonomic characters}

A taxonomic character is any attribute of an organism which can be evaluated, referring to structure, form or behaviour and which has two or more discontinuous states or conditions e.g. some morphological character of the flower such as shape of a petal, stamen number or length of a leaf. Strictly speaking, characters as such are abstract entities; it is their expressions or states which taxonomists deal with in their classifications. Plant characters are normally listed in the flora descriptions and the diagnostic ones are used in keys for identification. The value of a taxonomic character is increased if its biological significance has been determined but the significance of most characters is still unknown or poorly understood. Some characters are very stable and show little variation from individual to individual and from generation to generation. Such are those of the flower and fruit in angiosperms and the female cone in gymnosperms and which are used extensively in the identification and classification of these groups. Morphological features of vegetative organs, stems, leaves, bracts, etc. which tend to be much more variable and affected by the environment are important at levels below that of the 
species $\underline{i} . \underline{e}$. in describing intra-specific variation. Other important characters can be found in the chromosome complement, (especially number and karyotype), wood and pollen structure and in the chemistry of secondary plant products (see Sections 2.19 and 2.2).

\subsection{Classical taxonomic categories}

Botanical classification involves the assembling of plants into taxonomic groups (taxa) according to their presumed relationships. These groups are assembled into more inclusive groups and these into still more inclusive groups. The different levels of groups so produced are recognised as a series of categories which form the taxonomic hierarchy. The categories or levels are entirely artificial and subjective and have no basis in reality; they can only be defined by their position relative to other categories.

In classification the numbers of categories and relative rank of each are fixed by the International Code of Botanical Nomenclature (1972) and pose no problems; problems however do exist in deciding which characteristics to use in the delimitation of each category and in the appropriate taxonomic rank to be given to an organism or group of organisms. The formulation of groups or taxa is highly subjective because the characteristics may be of no value - or may not even exist - in the organisms of another series. Both the levels (or categories) and the groups that are assigned to them are named in the same way - genus, species, variety, etc. A taxon placed at the genus-level is called a genus; that at the species-level, a species, etc.

The categories of most concern to foresters and tree breeders involved in species and provenance trials are the genus, species, subspecies and variety and these will be dealt with in detail below. They are all long established and most have been in use since before the time of Linnaeus.

\subsection{Genus}

This is one of the two categories which forms the name of an organism and is a rather arbitrary category in the taxonomic hierarchy between the level of family and species. Genera consist of one or more closely related species and are defined mostly on characteristics of the flower and/or fruit. Thus all the species of pine (genus Pinus) have their needles in bundles (1-5) and the female cone is composed of woody (ovuliferous) scales and insignificant or rudimentary bract scales. Frequently genera are so distinct that they can be recognized easily $\underline{e} \cdot \mathrm{g}$. Eucalyptus, Cedrela, but the species which comprise them are often very difficult to distinguish.

\subsection{The species}

The species is now universally accepted as the basic unit of formal classification and it is to this that the binomial or name is attached (see below). It is unlike any other taxon in that it can be described and recognised without relation to any other category - all organisms 
belong to a species.

There has been, and still is, much discussion about the meaning of the term species and no universally "correct" definition satisfying all needs is possible. Yet, in general terms, everyone knows what a species is. To most people working with tropical trees where there is little or no knowledge of the reproductive biology of the individuals concerned, the species category is widely used for assemblages of individuals which share in common some morphological features of leaves, flowers and fruit and which are separable from similar assemblages by other correlated morphological discontinuities. Generally the populations comprising a species have a definable area of geographical distribution and possibly specific ecological requirements. The concept is, however, somewhat subjective since there is no general agreement on the degree of similarity between two plants or populations in order for them to be placed in the same species. It is usually accepted (but by no means proven) that individuals of similar morphology included within a species are potentially able to interbreed among themselves and to breed true, whereas breeding barriers, whether genetic, ethological or ecological, etc., exist between species. Hybrids under natural conditions are, therefore, not formed or are rare.

Field workers making collections of seeds for species and provenance trials should make sure that their parent material is correctly and accurately identified. This can usually be done by referring to a Flora of the area, State or Country from which it is obtained. Voucher botanical specimens of parent trees must be collected, together with adequate notes on place of origin, field characteristics, ecology and phenology, etc. Specimens should be deposited in a public herbarium or at a forest department where they can be referred to if the need arises. Where no complete or adequate Flora exists, as in several tropical countries, experts working on particular groups should be approached to assist with identification. Eucalyptus, Pinus, Quercus and Dipterocarpus are large genera in terms of numbers of species, with complex variation patterns and where specialist botanical knowledge is frequently necessary. Botanical material should be as complete as possible, i.e. with flowers or fruit (better both), together with adequate vegetative parts to give some idea of the overall range of variation (Leenhouts, 1968).

It is essential during trials that each species be kept apart and clearly labelled at all stages of the investigation, from first raising seedlings through initial planting out and later transport to the experimental site. Some species are so obviously different that no problem arises if mixing occurs, but in the case of the more closely related pines, which all look alike when immature and sterile, and still more in the case of different provenances of one species, clear and continuous labelling is imperative.

\subsection{The subspecies}

The subspecies is undoubtedly the most widely used and most important of the intraspecific taxonomic categories. However, it should never be used until the variation pattern of a species has been ascertained 
throughout its entire range of distribution. This taxon has been accepted as a segment of a species with a more or less distinct morphology, often showing intergradation of characters with the typical form. Many subspecies are distinguished by several small quantitative differences where any one character can break down individually. Present-day taxonomists normally use the term to cover a more or less regional facies of a species i.e. correlated morphological differences within a localized area. Thus before any variant is likely to be recognized as a subspecies the morphological differences between it and the typical representatives of the species must be seen to have some degree of broad geographical correlation. For instance, Corsican populations of the Austrian pine, Pinus nigra Arm. are now considered to be sufficiently distinct to warrant the rank of subspecies (subsp. laricio (Poir.) Maire). The remaining typical (continental) populations automatically become Pinus nigra subsp. nigra.

\subsection{Variety}

Unfortunately this taxon has been and still is employed in several different senses. To most taxonomists the term variety is usually taken to mean variants of a typical species which are not known to occur as distinct populations but appear sporadically in wild stands as single or isolated individuals. Uncorrelated colour variations of angiosperm flowers, peculiar growth forms, etc. are often given varietal names. They are frequently due to the influence of a single Mendelian gene, or the result of repeated associations of recessive genes. The term has also been used to describe the facies of a species with a very local range. Among conifers and other gymnosperms the term variety is apparently used in place of the more acceptable subspecies, e.g. Pinus caribaea var. caribaea Barr. \& Golf. The reason for this atypical usage appear to be mainly historical.

\subsection{Nomenclature}

One of the first tasks of biological science is to make meaningful generalizations about the organisms being dealt with - in our case trees - so that useful knowledge can be passed on from person to person and from generation to generation. Thus, after classification and identification of material during taxonomic work comes the process of allocating names to the groups produced.

We have already referred (2.13) to the taxonomic hierarchy and to those classical taxonomic categories which most concern foresters and tree breeders engaged in species and provenance trials. Latin plant names for the plants have been used throughout. Common or vernacular names are quite unsatisfactory for use in botanical nomenclature since the same name is often used in different senses to denote different kinds of organisms. (For instance in Mexico all pines are known as 'ocote', but in Central America 'ocote' usually refers to $\underline{P}$. oocarpa Schiede only.) There are besides, a large number of languages using many differing alphabets and scripts. For this reason the International Code of Botanical Nomenclature (1972) demands that all plant names shall be Latin in form, written in the Latin alphabet and subject to the rules of Latin grammar. 
The names of genera are uninomial (i.e. consist of one word) and are singular nouns always written with a capital letter $\underline{e} \cdot \underline{g}$. Pinus, Cedrela, Tectona, Eucalyptus. They can be of almost any origin.

The species name consists of two terms and is called the binomial. It is made up of the name of the genus in which the species is classified plus a second term - the epithet which is peculiar to the species - viz. Pinus kesiya, Triplochiton scleroxylon,etc. The epithet may be an adjective (in which case it must agree in gender with the generic name), or a noun. The specific epithet is normally written with a small initial letter and is never used on its own. In writing about a particular species the name (binomial) should be used in full once at the beginning of the text e.g. Pinus merkusii and may then be abbreviated subsequently to $\underline{P}$. merkusii so long as no confusion or ambiguity is likely to arise. It is general practice to print all latin names in italic script.

In botanical taxonomy several categories below the rank of species are recognized. The names of such infraspecific taxonomic groups consist of the name of the species in which they are classified, followed by a term peculiar to the taxon, but preceded by a word indicative of the rank e.g. Juniperus communis subsp. (subspecies) nana, Pinus caribaea var. (variety) bahamensis. These are in fact trinomial names and the insertion of words indicative of the rank is obligatory.

The instability of botanical nomenclature is well known and infuriating to the users of plant names. Unfortunately some name-changes are almost unavoidable because systems of classification are subject to change and ideas on generic and specific limits can be and are modified. Only a small proportion of recent alterations in names are due to the rigid interpretation of the Code* and very many are for scientific reasons alone.

The full citation of a botanical name contains a third element, the name of the author who first proposed it. This is often abbreviated: it should always be written after the epithet $\underline{e} . \underline{g}$. Pinus kesiya Royle ex Gordon, so that no confusion can occur.

If two or more names are considered to apply to the same plant, only one of them according to the Code, generally the oldest one, can be the name by which it is properly known. The other name(s) are known as synonyms and are placed after (or under) the correct one and preceded by the abbreviation syn., e.g. Cedrela odorata L. (syn. C. mexicana M.J. Roem.). The synonomy of a taxon is of the greatest importance since a considerable amount of information may be recorded in the literature under such synonymous names.

\subsection{The biological species}

We have stated above that taxonomists dealing with tropical woody

* It should be perhaps stated here that the International Code of Botanical Nomenclature (1972) has been set up in order to bring stability to plant nomenclature and attempts to avoid or reject names which cause ambiguity or confusion. Too much trouble cannot be taken in making sure that names are spelt correctly and are used in the proper manner. 
plants consider species to be groups of morphologically similar individuals that differ from other such groups. However, the question is continually asked whether such species represent real 'biological' units in nature, and whether the species category can be defined objectively. As more information becomes available on the genetic and ecological mechanisms governing the breeding behaviour of individuals and populations, these aspects are being accommodated more and more within the species concept. This has led to the development in the last 30 years or so of the socalled biological species. Other aspects included within this concept are a study of the variation of characteristics over large geographical areas, changes in these characteristics as populations adapt to environmental challenges or interact with other populations, and the integration of individuals into populations to form gene pools. We may thus define the biological species following Mayr (1963) as groups of actually or potentially interbreeding populations which are reproductively isolated from other such groups. The ability of individuals to exchange genes successfully i..e. to cross freely and produce fertile and viable progeny characterizes them as members of the same biological species, whereas inability is the mark of separate biological species. Morphological and other character differences are subordinate to this. Although the concept of the biological species will probably be almost impossible to apply in taxonomic studies on tropical tree species, some knowledge of the type of information mentioned above is vitally important when considering provenance testing and tree breeding. This is discussed below.

\subsection{Implications of the breeding system}

Some information on the breeding behaviour and gene flow in populations of temperate tree species is available, particularly for European hardwoods and conifers. Very many more detailed investigations on tropical trees are required, though chromosomal cytological studies particularly in important woody groups (once an almost barren area) have blossomed during the last decade (Jong and Lethbridge, 1967; Styles and Vosa, 1971; Mehra, 1972) producing interesting new information. Studies of pollinating types and mechanisms, pollen range, incompatibility systems and cytogenetics are now beginning by direct observations on trees in the forest. The work of the Organisation for Tropical Studies in Costa Rica is particularly important in this respect (Bawa, 1974). Ashton and co-workers in Malaysia are embarking on a similar project involving rain forest species (Ashton, 1976).

Workers in the forest should make all-out efforts to increase our biological knowledge of the timber trees they are concerned with by keeping note-books and recording information throughout the year, $\underline{i} . \underline{\text { e. }}$ phenology ( $f$ lowering times), pollen vectors, time of seed dispersal, etc. Dispersal agents are unknown for the majority of our economic species. This vital information is necessary if genetic improvement and breeding of timber trees and understanding of the patterns of gene flow and genetic differentiation within and between populations are to progress.

\subsection{Problems of uniparental reproduction}

If plants are apomictic (i.e. they produce seeds without fertilization), obligate self-pollinators (i.e. inbreeders) or they reproduce 
solely by asexual methods, then, by their very nature, they cannot form biological species. This type of reproduction produces complex variation patterns in all characters so that even the concept of the morphological (taxonomic) species is difficult to apply. Since apomicts do not form populations in the biological sense no gene exchange is possible and all individuals in a "population" appear morphologically similar often differing from other "populations" in slight (though constant) characteristics which may be difficult to recognize. Such "populations" are effectively pure lines and are genetically uniform. Taxonomy is difficult because such pure lines are often given the rank of species, though they in no way qualify for this status. Giving specific names to apomictic segregates is not to be contemplated nor encouraged.

Only a few tropical tree genera have so far been discovered with at least some apomictic species, e.g. Casuarina, Citrus, Mangifera and Alnus, but many others are suspected.

The extensive reproduction of timber species by cloning as in Populus is likely to give rise to taxonomic and nomenclatural problems, especially if hybridity is involved.

\subsection{Experimenta1 taxonomy}

The Swedish botanist Turesson was one of the first people to notice the different environmental tolerances of species and that different populations of the same species occupying distinctive habitats frequently showed morphological and physiological traits often of an adaptive nature. For many years he explored the phenotypic plasticity of single genotypes of local plants in a number of now famous transplant experiments. One of the main aims of his experiments was to see if differences shown by his plants were due to modifications of the same genotype as a result of direct environmental influences, or to the expression of different genotype combinations in the different habitats.

Turesson found that most of the plants which he collected from differing habitats retained their morphological and growth characteristics when brought into cultivation from the wild - thus refuting the idea that genetic variation within populations is largely governed by chance. The occurrence of widespread habitat-correlated variation supports the view that natural populations are subject to natural selection with well adapted genotypes being selected in each habitat. His investigations of the genetical differentiation of populations have now come to be known as experimental taxonomy, but he himself applied the term genecology to this relationship between the genetical compositions of populations and their environments.

The result of Turesson's work was the formulation of a series of experimental categories which he proposed in 1922 in an attempt to break away from the classical taxonomic groupings based almost exclusively on morphology. In this way taxa became framed around natural populations rather than selected specimens. The most useful of these experimental categories have proved to be the ecospecies and the ecotype - the essential criteria of which are ecological and genetical. These terms 
have been variously modified since the time of Turesson's original work, but his basic concepts still stand (Valentine and Löve, 1958).

\subsection{Ecospecies}

By definition the ecospecies is a sexually-reproducing population, the constituents of which produce vital and fertile descendants with each other but give rise to less vital or more or less sterile descendants when crossed with individuals of any other population. This implies free gene exchange within ecospecies but under natural conditions it may be impeded between different ecospecies.

Thus the two central American pines, Pinus oocarpa and $P$. caribaea may be thought of as good ecospecies in that, although their $\bar{d}$ istribution is sympatric over part of their range they generally occupy, differ ecological niches and do not interbreed (or hardly ever). In many respects the ecospecies may be allied to the taxonomic species; in other cases it may include two or more Linnean species which are able to interbreed when they come into contact, as in certain European herbs.

\subsection{Ecotype}

This was applied by Turesson to those products of the reaction between the genotype and the habitat which are found as a result of the selective action of the predominant factors of the habitat in sorting out those biotypes which are most suited to the specialized conditions and the selective elimination of those individuals which are unfitted. Thus, when similar complexes of environmental conditions exist within an area of a species, the same or essentially similar ecotypes can be expected to arise. This is of great significance in provenance research. There is no genetic barrier to exchange of genes between different ecotypes but they are frequently prevented from doing so by ecological or other barriers. Of more relevance today is the definition of Gregor (1939) who redefined the ecotype as a range of an ecocline.

Sometimes ecotypes are found to coincide with recognized orthodox taxonomic categories. If, for instance, they are localized geographically they may represent subspecies or, if very localized, varieties. Frequently, however, the adaptive features of the ecotype do not lend themselves to orthodox taxonomic treatment since they are often distinguished by characters which are not correlated with others, such as flowering time, seasonal growth rhythms, habitat and other physiological features.

This approach to the study of variation continued with the work of Clausen, Keck and Hiesey (1940) in a spectacular series of transplant experiments in the Rocky Mountains in California. More recently Bradshaw (1952) has studied ecotypic differentiation of a temperate grass (Agrostis) on mine and spoil-heaps containing toxic levels of heavy metals such as lead and zinc (in this case it is physiologically adaptive). It is not yet fully realized that by sampling populations of wide ranging species in provenance research and raising progeny under different environments, foresters and tree breeders are in fact contributing enormously to our understanding of Turessonian genecology. 
of particular significance to our understanding of variation, will be the series of large scale provenance trials of several tropical tree species now in progress, e.g. those of the Commonwealth Forestry Institute, 0xford (Pinus caribaea, $\underline{P}$. oocarpa, and Cedrela odorata) and of the Danish Government (Tectona grandis).

\subsection{Clines}

The fact that ecotypes have now been described in so many plant species suggests, perhaps, that all species in nature are represented by local races or populations which possess distinct morphology. This idea was first questioned by Langlet (1934) who pointed out that most habitat factors e.g. rainfall, temperature, soil type, etc. commonly vary in a continuous fashion and that in widespread species one might expect graded variation of morphological characters rather than discontinuous and discrete variation patterns.

This view received considerable support from the many European workers who were making intensive variation studies on temperate herbs and trees at the time, and Langlet was himself one of the first forest geneticists to describe a type of continuous variation in the European Pinus sylvestris L. In 1939, Huxley coined the useful term cline to describe these character variations which are related to environmental gradients. Thus a pattern of gradation in phenotypic (including physiologica1) characteristics associated with an ecological gradient is known as an ecocline, that with geographical factors, a topocline, etc. The term cline is not a taxonomic category, but rather a term to describe a particular type of continuous variation. Numerous published examples of clines exist in botanical and forestry literature relating particularly to temperate tree species. Clines can only be demonstrated convincingly by the careful sampling of contiguous populations of a species throughout its entire range, such as, in the topocline in Pinus strobus where Mergen (1963) has shown in N. America that leaf length and number of stomata decrease, whilst the number of resin ducts increase with latitude.

Published examples of clinal variation among tropical and subtropical forest trees are, as expected, more difficult to find; the convincing work of Barber (1955) on Eucalyptus in Tasmania deserves mention. He has demonstrated convincingly by experiment and field observations that a cline for glaucousness (as reflected in the amount of wax which develops on the twigs and leaves) exists in several species. Here clines in glaucousness are correlated with changes in frost activity - the more glaucous populations occurring in the more frosty localities. Wax is thus responsible for increasing frost resistance.

Another species, E. microtheca F.v. Muell. shows clinal variation in its type of bark, ranging continuously from completely smooth, decorticating "gum" to wholly rough bark. These observations call into question the usefulness of bark as a diagnostic taxonomic feature in species delimitation in the genus, and which taxonomists have for so long regarded as important (Barber, 1965).

Much of the variation encountered in forest trees is in fact clinal 
in nature and it is probable that the many clear-cut ecotypes described in wind pollinated, outbreeding tree species, especially the Coniferae, are parts of clines, their apparent distinctness being due to randomly chosen segments which are growing in different localities.

\subsection{Deme}

This system of terminology was introduced by Gilmour and Gregor (1939). Although frequently mentioned in theoretical textbooks it has received little support in practice. In this system the categories of formal taxonomy are rigorously excluded and the term deme refers to a neutral category to which a prefix is added denoting any group of individuals of a specified taxon. Thus a gamodeme is a deme composed of individuals which are so situated spatially and temporarily that within the limits of the breeding system all are able to interbreed (viz. a breeding population). An agamodeme is a deme composed of predominantly apomictic or asexually reproducing plants. Further prefixes can be added such as an ecogamodeme which is a breeding population in a specified ecological habitat. This could well be thought of as synonymous with the term provenance (see below). Species are considered to consist of a series of hologamodemes. The terminology has been used with some success in describing asexual populations (clones, etc.) but has been largely ignored by taxonomists.

\subsection{Provenance}

Provenance, with reference to clonal or seed material, is the geographical area and environment in which parent trees grew and within which their genetic constitution has been developed through artificial and/or natural selection. Although this purely forestry term has no place in the formal taxonomic hierarchy, it may, for natural, indigenous forest, and depending on the nature of the populations under consideration and the size and distinctness of their geographical area or ecology, be equivalent to an ecotype, a geographical race, a variety or perhaps more frequently to part of a cline. This is provided that some morphological or other traits can be recognized to characterize them. No system of latin nomenclature is applied. e.g. Pinus oocarpa "E1 Junquillo" refers here only to the provenance of those trees which occur in a limited area of Nicaragua and which will be subject to the local conditions of climate, soil, altitude, etc. prevailing there. It must be repeatedly stressed that it is essential to record the place of origin of all material (seeds, clones, etc.) used in species and provenance trials. (See Glossary in Appendix 1 and the paper by Jones and Burley, 1973, for distinction of provenance and origin.)

\subsection{Cultivar}

The international term cultivar denotes an assemblage of cultivated plants raised or maintained by man which is clearly distinguished by some characters (morphological, physiological, cytological, etc.) and which, when reproduced either sexually or asexually retains its distinguishing characters. The plants may be clones, self-fertilizing individuals or individuals reconstituted on each occasion by crossing. 
Botanical Nomenclature because they do not have a natural population structure, and they often grow in monospecific communities under entirely artificial conditions (Jeffrey, 1973). For this reason the botanical hierarchy of infraspecific categories is hardly applicable. They are thus subject to their own nomenclatural rules, embodied in a special Code. The range of variation of cultivars may be very narrow but it can also on the other hand be great since they are often very polymorphic, through the breakdown of isolating mechanisms or hybridity.

A cultivar is distinguished by a special epithet which is a nonlatinized or fancy name added to the usual binomial, e.g. Chamaecyparis lawsoniana cv. "Silver Queen". Cultivars of temperate ornamental trees have been grown for many years in arboreta and parks, some showing striking growth habits (fastigiate), or abnormal leaf forms (dissected), or colours (variegated). Such "abnormal" forms are nearly always reproducted by asexual means.

As a result of provenance trials, very many forest trees are being grown and bred as exotics in plantations, often far from their native habitat and country of origin. Where such trees are selected by man for a particular trait or characteristic which is sufficiently distinct, the individuals may be recognised and, if it is thought desirable, given a cultivar name. Cultivars of such important forest trees as Populus, Cryptomeria and Metasequoia are already well known, and clones of Cedrela and Triplochiton are being raised in West Africa. Cultivar names of forest trees if used consistently should be properly registered with a recognised registration authority.

\subsection{Modern methods of studying species}

The best classifications are those based on all possible sources of data. Normally, as we have pointed out already, morphology forms the basis of tropical plant taxonomy and will certainly continue to do so. However, in recent years new sources of taxonomic data and methods of assessing it have emerged. Among the data sources being investigated are micro-morphological features of seed, fruit and leaf surfaces as revealed by the scanning electron microscope; the assessment and value of chemical compounds produced and stored in plants including isoenzymes and amino acids (chemotaxonomy, see Section 2.2).

Computers are now being used more frequently to analyse multivariate data and complex patterns of variation. 
No other technical references are quoted in this Chapter because of the diversity of authors, species and chemical compounds that have been used. Detailed reviews of the literature have been given by Burley and Lever (1973) and Lever and Burley (1974).

Species and provenance trials are established in the field generally to determine the best populations for commercial planting by examining the extent and pattern of variation between and within species in survival and productivity. Even with fast growing tropical species it is often necessary to wait several years before reasonably safe conclusions can be drawn.

The performance of trees in growth chambers, greenhouses or nurseries has sometimes been correlated with their growth in the field (early tests; studies of juvenile-mature correlation) but reliability of the correlation is doubtful; it varies with species and usually only allows elimination of the worst $25-50 \%$ of the populations under test. This could be useful in a selective breeding programme when possibly several hundred progenies must be compared. However, in species and provenance research it is necessary to identify the best one or two populations from a reasonably small initial number $(10-30$ or 40$)$. In such cases some form of risk analysis is necessary.

It is also desirable to determine the pattern of genetic variation between populations more precisely than is commonly possible with productive traits such as timber volume. This is to determine the evolutionary, genecological and taxonomic status of the species, and particularly to predict the performance of unknown or untested populations. If a species in a provenance trial exhibits a continuous (clina1) pattern of variation between populations related to some characteristics of the seed source (e. $g$. latitude, altitude, temperature), the performance of populations not included in the trial may be predicted by regression methods. If the species exhibits discontinuous (ecotypic or random) variation such prediction is not possible and there is no safe alternative to adequate field testing of all seed sources.

There are many anatomical, biochemica1, morphological and physiological traits that can be measured far more precisely than growth parameters, whether in the field or under controlled environmental conditions. Although these may not be directly correlated with growth they give a good indication of the amount and pattern of genetic variability existing in the species.

In addition to obtaining knowledge of relative mean productivity, we need to know the amount of variability in the populations to plan rational breeding strategies and to guard against future decrease of the genetic base. Further, some programmes of tree introduction and improvement are concerned with properties other than timber per se (such as quantity and and quality of resins, essential oils, rubber or sugar) or additionally 
with resistance to adverse factors that reduce productivity (such as cold, snow damage, wind, drought, heat, air pollution, flooding, salinity, diseases and insect pests).

Fully comprehensive species and provenance studies include studies of all such productive traits together with taxonomic characteristics in the source populations, in field experiments and in controlled environments. Together the studies in the natural range and in comparative experiments form the subject known variously as biosystematics, experimental taxonomy, genecology, transplant studies or provenance research.

Examination of the same traits in a set of provenances grown in several sites or environments provides estimates of genotype-environment interaction effects. Such effects contribute largely to the lack of genetic gain obtained by selection of species or provenances that are hoped to perform well on all sites; interactions add to costs because they imply that different populations should be used on each different site type.

Several biochemical methods now available are applicable to the evaluation of genetic differences between and within tree populations. In many cases evidence of such variation may be obtained at an early stage of species and provenance research, either in the natural stands or in young trials. Not all methods could or should be applied necessarily to all experiments; nevertheless the methods summarized in this Section are believed to be particularly promising aids to provenance research and to understanding the diversity of tree species. In many cases they have the desirable property of being relatively unaffected by environment, in comparison with metric survival and productive characters, and can therefore be examined in natural populations in advance of field provenance testing (which is, effectively, a form of progeny testing to evaluate the genetic control of properties in species and provenance populations).

Many countries lack the equipment, staff, time and funds to include biochemical studies in their forestry research programmes. However, several international agencies and institutes, particularly those coordinating international provenance trials and gene centres, should be prepared to provide centralized biochemical services once the value of the techniques has been demonstrated. The biochemical approach has been neglected but it is now becoming more widely used as new sophisticated equipment and techniques are developed at reasonable cost. Also the education of foresters now frequently includes a knowledge of such laboratory techniques. A Working Party of IUFRO ( 2.04 .5$)$ has been established to foster international cooperation and exchange of information on biochemical methods.

\subsection{Topics in which biochemical methods are applicable}

\subsection{Studies of inter- and intra-specific variation}

For many species, particularly in the tropics, the distribution and taxonomic relationships of different species are inadequately known. Inter-specific taxonomic variation can only be assessed by comparison with 
the extent of intra-specific variation and classically the taxonomist studies morphological traits, particularly the structure of flowers and leaves, that are sufficiently constant within a species to permit discrimination between species. However, in species that are wide-ranging, unexplored, or physically and reproductively merging with others, the additional information supplied by chemical characters may be valuable. Populations may be separable on the basis of the mean content of a particular compound or on the basis of the frequency of individual trees possessing particular chemical characteristics.

The taxonomist should include studies of morphological and productive traits in transplant experiments to examine the genetic control of such traits and to confirm his classification; however, the advantage of several chemical compounds is that either presence and absence or their relative amounts, appear to be under direct genetic control; they can therefore be evaluated in natural forest, although effects of age, season and position of sampling may be important. Among important tree species such compounds include conifer terpenes (such as the pinenes, delta-3-carene, myrcene, longifolene, caryophyllene and limonene). Phenolics (…g. flavanones, stilbenes, leucoanthocyanins) also provide useful information at different taxonomic levels in pines and eucalypts. Complete reviews of compounds that have been used taxonomically are given in the papers mentioned above. The use of biochemical characteristics is particularly important when morphological characters are lacking or difficult to obtain or assess, e..g. in seed source identification (see 2.225 below).

\subsection{Estimation of genetic variation within populations}

Even if a population is acceptable for its mean performance it may still be unsuitable for commercial introduction because of its genetic structure, particularly the lack of genetic variation. Derived provenances (í.e. seed sources grown in exotic conditions) commonly, and natural provenances occasionally, arise from one or a few parent trees and hence contain a small amount of genetic variation. Alternatively they may arise from several to many trees that are closely related and hence have high inbreeding coefficients. In either case the genetic advance obtainable by subsequent selective breeding is reduced. Biochemical characters that are controlled directly ( $\underline{\text { e.g. }}$. terpenes and enzymes) may give direct estimates of the population genetic composition and also indicate the relationships between adjacent populations, including the extent of hybridization or introgression. Estimates of population variability, together with knowledge of the natural breeding system, are essential in assessing the need for and methods of genetic conservation.

\subsection{Identification of genetic markers and indirect selection}

When the 'best' species and provenance have been selected it is usual to establish plantations on either the pilot or commercial scale (see Chapters 1 and 5). This is followed by selective breeding in which superior phenotypes are selected, tested and mated with each other in various combinations to produce subsequent, genetically improved stock. The tree breeder commonly needs to check the progress of his breeding strategy and 
the genetic composition of his breeding populations. Genetic markers are necessary for this purpose; these include visible, physiological genetic mutants (such as albinism and some leaf abnormalities) or they can be biochemical.

The possibility of using biochemical traits in indirect selection of population or individual genotypes rests on establishing a correlation between the chemical compound actually selected for and the productive trait that it is wished to incorporate in the afforestation and breeding programme. The most common correlation is between chemical constituents and a component of production such as survival, or resistance to adverse, climatic factors and pests or diseases. Again conifer terpenes and hardwood tannins are good examples. Studies of isoenzymes may facilitate the prediction or explanation of genotype-environment interactions.

\subsection{Evaluation of biochemical products}

In some afforestation programmes the objective is not only protection, amenity or timber; it may be the production of commercially important biochemical compounds. Differences between species and provenances occur for such products as sugar (in maple, Acer species), eucalyptus essential oils and pine turpentines. Analysis is necessary to determine the quantity, quality and chemical composition of such natural products. Alternatively some trees produce undesirable compounds such as kinos (gum) in eucalypts or other chemical compounds that may affect the utilisation of the crop, e.g. the thermal properties of firewood, impregnation, painting or conversion of timber, and pulping properties for paper and boards. In all of these, differences may be expected between populations.

\subsection{Seed source identification}

Many tons of tree seed are traded nationally and internationally every year, commonly without adequate information about the seed source (seed certification; see Jones and Burley, 1973). The history of exotic forestry is filled with examples of plantations in which the growth has been either very good or very bad yet the reimportation of seed from the same source cannot be guaranteed or avoided. Similarly many species and provenance trials include populations that cannot be interpreted unequivocally.

The seed sources for both seeds and plantations are difficult to identify from morphological and physiological tests. However, some chemical compounds that are under rigid genetic control may be useful, particularly terpenes and enzymes. This may be expandable into techniques for routine seed certification quite apart from seed for species and provenance trials.

\subsection{Biochemical characters}

Several techniques are now available for the rapid determination of identities and quantities of chemical compounds. These are often applicable to the evaluation of differences between populations of trees. In 
many cases evidence of inter- and intra-population variation may be obtained at an early stage of species and provenance research.

\subsection{Mineral nutrients}

Chemical and spectographic analysis of mineral nutrients in foliage have frequently shown interprovenance differences in nutrient concentrations. Generally the importance of mineral nutrient analysis lies in the evaluation of deficiencies of soil nutrients. However, in evaluating such soil deficiencies it is essential to remember the possibility of inter-provenance differences in the relationship between foliage nutrients and tree growth parameters (í.e. efficiency).

\subsection{Organic compounds}

\section{Primary metabolites}

These are usually compounds with low molecular weights; they take part in fundamental metabolism and are not normally stored. Examples are the protein amino acids and simple sugars. Inter-provenance differences in seeds have been found to be associated with climate of seed source in some species but large variations occur in different parts of the plant.

\section{Secondary metabolites}

These compounds also have relatively low molecular weights, but, unlike the primary metabolites, they are often accumulated either in living cells or else deposited in specialised ducts (e.g. coniferous resin ducts) or dead tissues (e.g. bark and heartwood). They are frequently termed "waste products" or "metabolic by-products"; whilst some probably do represent the terminal product of particular metabolic pathways, others may be intermediates in unknown or poorly understood pathways. Examples include lipids, alkanes, waxes, alkaloids, terpenoids, polyols and polyphenols. Most work on intra-specific variation of trees has been concentrated on terpenes, particularly coniferous monoterpenes.

\section{Macromolecules}

Two broad groups of macromolecules can be recognised. The first type (monomer and linkage invariant) is characterised by compounds such as cellulose and chitin in which the monomeric components are identical in different organisms. The second type (monomer and/or linkage variable) is represented by proteins and nucleic acids which are polymers of a limited number of monomers that show a considerable range of variation.

The macromolecules of most obvious interest to the forester are celluloses and hemicelluloses. There are important differences between populations in cellulose yield but not in chemical composition. Nucleic acids, and particularly DNA, also appear to vary in yield rather than in composition. On the other hand, seed and leaf proteins, particularly isoenzymes, differ between populations. Since they give a direct indication of an individual's genotype, they are very promising for studies of genetic variation at the population and individual level, and for seed 
source identification. Although its applicability needs to be determined for many more species, the analysis of isoenzymes represents one of the major potential contributions of biochemical studies to forest management.

\subsection{Techniques}

Mineral nutrients are analysed by standard gravimetric methods or by spectrographic techniques.

Recently techniques have been developed that permit rapid and accurate comparison to be made of the organic molecular characteristics of different populations. Among these the most important are quantitative chromatographic procedures such as thin-layer chromatography and gas-1iqud chromatography. These are invaluable in the separation, identification and quantification of individual components in extracts of both primary and secondary metabolites. Individual components are also identified by spectrographic techniques (including atomic absorption, infra-red, ultraviolet, resonance and mass spectrometry).

Nucleic acids have been studied by three main analytical techniques:-

(i) Determination of base composition by thermal denaturation or density gradient centrifugation.

(ii) DNA/DNA or DNA/RNA annealing or hybridization.

(iii) Quantitative variation of DNA per cell by microspectrophotometry.

In studies of proteins the most frequently used technique is probably that of electrophoresis on starch or acrylamide gels. Following electrophoretic separation of protein extracts, gels can be stained to show either the total protein pattern (electrophoregram) or else enzyme activity (zymogram). The description of multiple molecular forms of many enzymes (isoenzymes), reflecting allelic variants of given loci, has been largely responsible for the rapid advance in the use of protein characteristics in taxonomic and genetic investigations. Serological techniques have also been used with tree species to assess relatedness between antigenic preparations of different populations. The techniques are varied but generally too complex for most provenance research. 
Seed procurement for species trials and provenance research calls for cooperation between workers in different fields of forest research and administration, and quite often in different parts of the world. The techniques of collection and handling vary according to the nature of the species and the conditions under which it is found. Very often when dealing with tropical species precise information is lacking on the natural distribution, variability, flowering and fruiting times. There are many uncertainties regarding access, travel and transport of material, which can only be resolved when the collection is in progress. The amount of seed collected, and the selection of the areas, stands and trees to be included in the collection, may be to a large extent determined by local conditions at the time. In these circumstances a rigid manual of procedure, which prescribes precisely where, when and how a collection is to be made, is inappropriate. A more flexible approach is needed, that will permit the most effective use of the allocated resources of staff and equipment, by taking advantage of the opportunities that are actually found. Expeditions to explore and collect from the natural forests can be very expensive involving long and costly travel and transport of equipment. In remote areas there is likely to be material of great interest and potential value and it would be wasteful to return empty handed through too narrow a definition of aims and procedure.

The cost of the subsequent research, using the material collected, can also be very high. Major provenance trials, occupying large areas and demanding high standards of execution and documentation, are expensive to establish and maintain. Precise comparisons between many samples of material under carefully controlled conditions in glasshouse or laboratory can involve much valuable time and equipment. It is therefore essential that the nature of the material collected and the information provided (e.g. provenance seed collections and site data) should be compatible with the aims of the research and also that the degree of control over the material at all stages from collection to final use should be adequate to ensure that it is in fact what it is said to be. These are the simple rules for seed procurement. Almost any material, even a few seeds from a single tree, may serve to provide valuable experimental information if it is properly identified and documented. Seed intended for provenance trials must of course be collected from a sufficient number of trees to be properly representative of the population sampled. Much additional information may be obtained if the seed of individual trees is kept separately. Such a procedure involves extra cost at all stages from collection onwards and this cost must be weighed against the benefits to be gained from this or other alternative procedures.

Very rarely in international provenance research is the seed user able to collect the seed personally. He must therefore ensure that his objectives are clearly understood by those responsible for collecting and handling the seed, so that they know as exactly as possible what material and what information they should aim to provide. It is helpful for the user to bear in mind the difficulties facing the collector and not to ask for too much material, or for unnecessary detail and precision in the 
collection. A single collecting expedition may be able to undertake separate collections for different objectives or to collect seed in such a way that it is suitable for a variety of objectives. For this the different requests for seed must be prepared well in advance, to allow time for planning and co-ordination of all the necessary operations.

This section is arranged simply as a check list of the successive steps which should be followed, or at least considered, when planning seed procurement.

\subsection{Objectives of seed procurement}

Although it may be possible to meet several different objectives in the course of one collection programme it is important to decide beforehand which should be given priority, in case it is necessary to choose between them for such reasons as lack of time or funds. There is always some conflict between the need to cover many sites and the desirability of collecting large quantities of seed, or of making detailed studies at individual sites, since time at least is always limited.

\subsection{Seed source trials (provenance trials)}

Field trials to select the most appropriate seed source for a particular set of growing conditions and end use requirements call for seed collections that are representative of the population from which they are collected. The exact location of the seed source must be recorded together with other information on the site and the population sampled. The number of trees included in the collection should be recorded, although for large numbers an approximate count is sufficient. Seed from all the trees may be combined and it is not essential to maintain the separate identity of individual trees or of their seed once the collection has been completed. (Although special collection is usually required for most seed sources in a trial, seed from large commercial collections of well identified sources may also be included and individual countries using exotic species should include any locally produced seed as a standard or control.)

\subsection{Population improvement}

When collecting seed for provenance trials it has sometimes been the practice to select only the best looking phenotypes. The selection of individual phenotypes can be a powerful tool in population improvement, particularly when practised in well managed plantations of known history, and confirmed by study of the progeny. In some circumstances it may also be useful in natural stands, although when seed is transferred to other geographical and ecological situations the result of such selection is difficult to predict because of the frequent occurrence of genotype-environment interaction. This practice should not be confused with, nor regarded as equivalent to the collection of representative samples of seed for seed source trials (provenance trials). (See Ledig, 1974.) 


\subsection{Seed research}

Some problems in seed handling and seed storage (e. viability or prolonged dormancy) may be solved by study of bulk seed lots such as may be used for seed source trials. However, special collections are sometimes needed so that the exact conditions at all stages of harvesting and handling, and perhaps the identity of the parent tree, can be known exactly.

\subsection{Gene conservation}

Exploration of the natural range of a species for provenance research may reveal that some populations are seriously endangered through clearance of the forest. Where conservation in situ cannot be guaranteed it may be necessary to collect seed to establish gene conservation plantations elsewhere (Bouvare1, 1970). Seed collected for seed source trials may be suitable for this, provided that it is collected from a large number of trees. Large quantities of seed will be needed, in order to establish plantations in several sites, each large enough to serve as the base for selection and breeding, without the later risk of inbreeding depression.

\subsection{Planning seed procurement}

Planning of comprehensive seed collections for provenance research should start several years before the trials are established, to allow for preliminary exploration, the possible occurrence of poor seed years and the frequent need to collect for two or more years in order to cover the entire range. It is also advisable to give time for co-ordination or collection expeditions through FAO. An early contact should be made by writing to the Chief, Forest Management Division, FAO, Via delle Terme di Caracalla, 00100 Rome, Italy. Plans of seed collecting expeditions and similar activities are published in a newsletter - Forest Genetic Resources Information - and by this means more effective co-ordination of action is possible. These contacts may enable interested parties to obtain suitable samples of seed by request, without undertaking costly expeditions.

Even when seed is obtained through requests to another organisation, such as a local forest service, it is essential to make the request at least a year and preferably two years before the seed is to be sown. Although some forest services make regular collections of certain species the demand for these may be so heavy that only a few requests can be met each year. For species less in demand special arrangements are likely to be needed, which will require planning in advance.

\subsection{Survey of information on species and areas for collection}

A 1iterature search may provide some information on the extent of the natural range of the species, and the general pattern of its distribution. This information should then be plotted on maps. If there are records of flowering and fruiting times in different parts of the range, or evidence of patterns of variation within the species that may help to distinguish different populations, some of this information can be indicated on the map. This will help to decide the location of collection sites. Natural 
stands of a given species have usually become adapted over long periods to the precise environment in which they are found (Pryor, 1963). Variation in the environment, particularly, latitude, climate and elevation, will therefore help to decide the broad pattern of sampling that is desirable (Callaham, 1964). These factors also affect the actual collection, through their influence on travelling and working conditions.

Maps of the main communications systems, such as roads, rivers, railways and airstrips are the other main requirement. Much of the necessary detailed information may only be available through personal contact with local officials and collaborators, or even in some cases by actual reconnaissance.

\subsection{Contacting officials and collaborators}

Most countries have regulations governing collection, export, introduction and perhaps movement of seed. Official permits may be required for any of these procedures, and in some cases separate permits are needed for each collection site and for each individual seed lot exported or introduced. Airlines may require evidence that official regulations in the country of distribution have been complied with before they will accept seed for transporting. Customs officials and plant health authorities may seriously delay or even destroy seed if the full entry procedures are not followed. For some seed extra delays of even a few days in transmission can be fatal.

The official contacts with the forest services in the countries concerned will provide much essential information. The FAO Directory on forest tree seed (FAO, 1975) gives contact addresses for particular species, by countries. As plantation programmes increase in size and number more forest services are developing their own seed section. The widespread use of exotic species stimulates interest in exchange of information and seed. However, many forest services are still seriously understaffed and short of funds. In developing countries information on particular areas or species may be lacking and not easily obtained, owing to the deficiency of maps, roads, meteorological stations and so on. To avoid prolonged delays, or costly failures at a later stage, a reconnaissance may be needed. Such action will be welcomed by the local forest service if it is assured of free access to all information gathered at this and all later stages of exploration and seed collection.

\subsection{Field reconnaissance}

Within its own country a forest service can obtain much basis information on species' distributions, flowering and fruiting times and so on, through regular observations by field staff primarily engaged on other duties. This recurrent method should be strongly encouraged in tropical forests where knowledge of many of the species is very limited. For species which fruit only at intervals of several years, or which have a limited period of only a few days during which mature seed can be readily collected, the timing of seed collection is all important. The main aims of a reconnaissance are to determine whether seed production is likely to be sufficient to justify a costly collecting expedition, and also to determine the best sequence in which to collect from the various sites, bearing 
in mind both the variation in fruiting time and the practical problems of access and travel. At the same time information on variation within the species, and in its environment, at different points in the natural range, may be gathered.

\subsection{Timing of seed collection}

It is preferable to collect only in a year of abundant seed production since this offers much greater freedom of action in the choice of stands and trees, as well as the possibility of obtaining more seed for a given cost. Crop assessments made a few months before harvest, by reliable and experienced observers, are useful and can be progressively improved in accuracy if they are later compared with the actual quantities of seed obtained. Visual assessments from the ground can be very imprecise and it is often preferable to climb one tree in a group and make an estimate based on each climbed tree and its immediate neighbours. By this means specimens can also be collected that will help to determine the stage of development of the crop and thus the timing of the collection.

When attempting to collect provenance samples over a wide range the total time allowed by the length of the fruiting season can be a severe constraint. Determination of the most appropriate sequence of collecting sites, to take advantage both of differences in fruiting time and also the available means of transport between sites, can be a complex problem. Adverse climatic conditions may greatly alter the time needed at a given site, by hampering travel, collection and seed extraction. The preparation of alternative plans to cover such contingencies is particularly important when synchronization of work between separate collection teams is needed. All parties should know in advance what alternative programme will be adopted if the timing of the preferred programme cannot be followed.

\subsection{Choice of tactics and methods for seed collection}

At this stage in the procedure all available information will have been collected on which to decide when and how it should be organised, and also the resources of staff, equipment and funds that will be needed. Very often neither the resources, nor the information are sufficient to complete a range-wide provenance collection in one year. In these circumstances there are advantages in collecting from widely scattered points across the entire range during the first year, thus gathering much additional information needed for more detailed sampling later. This procedure may also reveal some populations in danger of extinction, which can then be given priority for conservation either in situ or by collection of reproductive material.

If conditions throughout the range are sufficiently well known in advance to decide on first priority areas it may be more efficient to cover only a selected part thoroughly in the first year, thus making some saving in time and funds spent on travelling. If felling operations are known to be planned for some areas this can be taken into account, and the opportunity taken to collect seed in larger quantity, or at reduced cost. For species which seed freely only at long intervals, advance evidence of an exceptionally good seed year may be used to obtain extra staff and funds 
to increase the capability, and perhaps cover the entire range in the one year. In such circumstances this is likely to be the most efficient procedure if the resources are available.

Decisions on equipment to be used must be taken at this stage, if long delays in purchase or delivery are likely. In addition to actual collection equipment, provision of vehicles for travelling and transport may be needed. Local transport services and hired vehicles are frequently unreliable and once an expedition is in the field, with limited time available, delays in transport can be very costly. A list of equipment that may be needed is given in Appendix 2.1 .

\subsection{Planning collection of correlated material and data}

Some correlated material, such as herbarium voucher specimens of trees from which the seed is obtained, must be collected at every site. It is desirable to illustrate the range of variation within each population by collecting a suitable series of specimens but for many highly variable wild populations this could lead to rapid accumulation of unmanageable quantities of material. In practice it is necessary to restrict the collection of herbarium material to only a few individuals in each population, bearing in mind that some collection of other vegetation may be desirable, if the main components of the association cannot be identified in the field. Notes and photographs can be used to supplement the herbarium material and if seed extraction is carried out on the site a photograph of a collection of the fruits can be useful to illustrate the range of variation.

Bearing in mind the cost of access to the areas visited the opportunity should be taken to collect other material of value for research that may have been requested, such as wood specimens or resin samples (see also Chapters 2 and 6 ). Special equipment for collecting and storing this material, such as specimen bottles, increment borers, storage solutions, refrigerated or insulated containers, may be needed. The additional research material may usually be collected while seed is being gathered, without loss of time, but when liquid samples such as resin are collected by extraction from the tree it may be necessary to plan the sequence of operations to allow sufficient time between affixing and removing the collecting bottles for the specimen to accumulate.

The field data to be collected must be decided in advance and the use of prepared forms is often advisable to ensure full and equal coverage of all sites. (See Appendix 2.2 for example.)

\subsection{Planning seed handling, transport and storage}

The period between the collection of the seed and its arrival in the seed laboratories where it can be properly cared for is of critical importance in its effect on viability and vigour. Careful planning is needed to provide the most favourable conditions practicable, and to reduce the transit time as far as possible.

At the collection site temporary shelters may be need to give 
protection from rain and surface water. Conditions in tropical forests, where temperatures and humidities are high, are very unfavourable for seed storage, even for limited periods, and when large quantities of fresh material are kept together there is danger of decomposition. Pests and diseases may build up rapidly in stored material and in some circumstances the use of insecticidal or fungicidal dusts may be recommended. For many species the seed must be extracted from the fruit by drying and, if this can be done at the collection site, transport and storage problems can be greatly reduced. This will involve spending a longer period at each site than is needed for actual collection, and the most efficient way to arrange this may be to leave one or two workers only, to look after the material during extraction, while the remainder of the collecting team moves to a new site. If a number of separate collections have been brought together for storage and extraction, such as collections from individual trees whose identity is to be maintained, the problems of handling are increased and a much higher degree of control will have to be maintained, under the supervision of a senior member of the collecting team, throughout the extracting process.

Storage for 1 imited periods may be needed at several stages in the transport of the seed but advance preparations to ensure that all documentation has been prepared, that transport is available, and that the recipients at each stage are ready to act, can greatly reduce the overall time spent in transit and prevent unnecessary exposure of the seed to harmful conditions, such as intense heat and damp.

\subsection{Selection of staff}

Collection of seed for provenance research demands frequent and rapid movement from site to site. Means of transport are often limited in size e.g. to a single four-wheel drive vehicle, a light aircraft or a powered canoe. In these circumstances small teams have greater freedom of action. The essential labour force is normally two tree climbers and four to five labourers, to clear vegetation, collect the fruits as they are dropped from the tree, carry the collected material and equipment and to look after the camp, the stored material and the seed during extraction. Whenever possible such "unskilled" labourers should be recruited in the area of operation since they may also serve as skilled guides and interpreters.

An expedition may be composed of several collection teams under one leader. The exploration and sampling of wild populations is not merely a technical matter of seed collecting and seed handling but involves a conscious effort to understand the ecological basis of the distribution, and to gather a wide range of data and material. The work must often be done under difficult and uncomfortable conditions, with frequent occurrence of unforeseen problems which require improvised solutions and the alteration of previous plans. In these circumstances it is vitally important that the expedition leader should have a good knowledge of, and above all a keen interest in the species concerned. An enthusiast is more likely to continue effective work in the face of obstacles and to encourage a similar approach in the other members of the party. 
Expertise and experience in seed collection methods are also important, and especially so in the leaders of the individual collection teams. An effective combination is for the overall leader of the expedition to be a specialist in the species under study and for the individual team leaders to be specialists in the techniques of seed collection and seed handling. If the overall leader also has the technical expertise it may be possible in some cases for him to recruit and train team leaders locally in the area concerned. However, any forest service or research institute engaged on exploration and seed collection of several species over a number of years will need a permanent staff of specialists to act as team leaders.

When expeditions are undertaken in a foreign country, perhaps with a foreign language, an understanding of the society, customs and language also plays a very important part in the success of the programme.

Early application for documents such as entry visas, work permits and health certificates is advisable since there can be long delays in the issue of permits which require approval in the country to be visited. This also applies to importation of some items of personal equipment, particularly firearms, if these are considered necessary.

\subsection{Organisation of seed procurement}

The start of field operations marks the end of the first phase of planning the collection, but in most cases much detailed planning still remains to be done as additional information is collected on the species, its distribution and the conditions in the different parts of its range, in the course of the field operations.

\subsection{Delivery of staff and equipment}

As much as possible of the essential equipment should be carried with the team, even if this involves extra expense on air freight, since delay in starting work after arrival due to delayed equipment can be very costly of both funds and the limited time available. All staff and equipment should be in the region for collection at least two weeks before the start of actual operations. When there is uncertainty about the time to harvest the seed, or when local recruitment and training of staff is planned, the team leader will need to arrive at least one month earlier, unless local collaborators are available to assist.

Insurance of equipment against loss or damage and of personnel against accidental injury or illness is strongly recommended.

\subsection{Exploration}

The information gathered in the first planning stage will have decided the starting point and a tentative sequence for the collection programme. The first stage of exploration will be to discover what changes may have been made in communication systems (new roads, airline schedules, etc.), development plans (including forest exploitation or clearances), areas of political unrest and so on which would directly 
affect the planned programme. At the same time local enquiries may provide more information on the species' distribution, the pattern of climatic variation within its range, variations in fruiting time and other factors which may influence the extent and sequence of sampling. Access to aerial photographs of the proposed collection areas may be possible with the assistance of the local forest service and this will greatly assist the detailed planning.

In many cases aerial photographs and reliable maps are not available and some time must be spent in reconnaissance of the first area in which collection is to be made before actual collection can be started. Once this had been done, and the necessary data on the site have been recorded, the expedition leader may be able to undertake reconnaissance of the next area, while the first seed collection is in progress. Such a reconnaissance may reveal that a proposed area is unsuitable and thus may save time and expense in moving an entire team unnecessarily.

Clinal patterns of variation have been observed in many tree species related to factors of the environment which vary along a gradient, such as day length or temperature. This concept has been usefully employed in planning provenance exploration. For range-wide provenance sampling (see Chapter 5) the collection sites are likely to be widely separated. The aim will be to sample at intervals along the major environmental gradients, such as latitude, rainfall and elevation, and to include isolated populations and those at the extremes of the geographical and ecological ranges. During this phase of work much additional information will be gained on ecological conditions, phenotypic variation and flowering and fruiting times that will permit greater precision in sampling at a later stage.

\subsection{Selection of stands and trees}

\section{Stands}

For a first range-wide provenance test at least five or six collection sites will be needed, in order to sample both the limits and the centre of the geographical range. If the distribution is continuous, and the total area covered by the species roughly symmetrical, the sites might be located on a grid pattern, at regular intervals. If it is a long, narrow band, parallel to major environmental gradients such as latitude or rainfal1, then sampling at intervals of half a degree or up to two degrees of latitude might be undertaken, depending on the total distance to be covered. However in many cases the situation is more complex, with local variations in climate, large discontinuities in the distribution due to mountain ranges, river valleys or sea, and perhaps some evidence of subspecific variation in the species in different parts of its range. The direction of migration of a species as it evolved and became separated from its nearest relatives may also be taken into consideration. For example the tropical pines in Central America have migrated southwards from a centre of diversity in the Mexican region, the migration in this case being roughly parallel to the gradient of decreasing latitude.

In many tropical countries the main communication systems, such as 
roads, rivers, and airways, are unevenly distributed, and access to some parts of the species' range will be more difficult and more costly of the limited time and funds, than to others. Even where sampling on a regular grid or strip pattern is indicated therefore it is unlikely to be practicable. Deliberate selection of certain areas is necessary, based on the objectives of the seed collection, the available information on variation within the species and variation in its environment, and the practical limitations imposed by local conditions and available resources.

Having decided the approximate location of the collection sites the actual stands must be determined. Good, recent aerial photographs are of the greatest value in this task and can save days of ground reconnaissance. The stands selected should be representative of that part of the species' range and should be sufficiently large and well stocked to provide satisfactory breeding conditions. In some cases the species may be present in relatively small but linked groups, partially separated by other vegetation, by rock outcrops, seasonal swamp, etc. Many tropical species may be found only as single trees in a mixed forest, widely separated from the nearest breeding specimen of the same species (and they may be self-pollinating). In such cases there is no actual "stand" to be considered and collection is made from the individual trees and the seed either kept separately or merged by sites, depending on the objectives. Detailed aerial photographs which permit identification of the species concerned are helpful.

At the extremes of the ecological range, where the species encounters limiting conditions such as drought, seasonal swamp, severe frost or extreme heat, the appearance of the stands is likely to be much poorer than in the centre of the range, where it is well adapted to the conditions. However, the populations which have evolved under such environmental stresses may have inherent qualities of great potential value despite their relatively poor phenotypic appearance.

\section{Trees}

For seed source trials and even more for gene conservation, it is desirable to capture as much as possible of the potentially valuable genetic variation within the population. In the absence of any estimate of population variances it is best to collect from relatively large numbers of trees at each site, avoiding immediate neighbours. Callaham (1964) has suggested 5-10 individuals in homogeneous populations and 25-50 trees in heterogeneous populations. Whenever possible it is preferable to follow this second prescription and to collect from not less than 25-30 trees. Having reached a site and located a suitable stand, the extra time spent in climbing 50 rather than 20 trees is small in comparison with the time spent in reaching the areas, reconnaissance, clearing tracks, extracting the seed and so on. For species which produce small crops per tree the gain in material will also be valuable. For very prolific seeders, if the total weight of seed is much greater than can be handled, the amount collected from each tree can be reduced proportionately.

Ideally the selection of the seed trees might be done randomly, but stratified to ensure equal representation of all classes of tree in the total sample (Callaham, 1964; Bennett, 1970). Such a strictly random 
sampling procedure would be difficult to follow in many areas of natural forest, where the stocking and terrain are often very irregular. In practice some degree of judgement in selecting trees to be climbed is inevitable, to avoid diseased and deformed specimens, those with very few fruits and so on. The method frequently practised is to collect from dominant and co-dominant trees at intervals of about 100 metres apart, to avoid as far as possible the inclusion of likely half-sibs in the collection. This is probably the best general rule to follow (Callaham, 1964). The deliberate selection of only those trees with certain desirable characteristics in the phenotype (equivalent to "candidate plus trees") is not recommended for provenance trials, although it may be done for other objectives. Such deliberate reduction in the apparent variability, if it is effective, may not necessarily prove beneficial when the material is transferred to a different environment. Nevertheless certain important characters which have been shown to have a high heritability in several species, such as stem straightness, must command the interest of practising foresters. The deliberate inclusion of the exceptionally straight and well formed trees in the collection is justified, provided that this biased sample is used to supplement the representative collection.

When two climbers only are working in a team it is quite easy for the team leader to range widely ahead of them, selecting the trees and attaching a removable marker. These markers are then collected by the tree climber and serve both as a tally of the number of trees and a check that none has been missed out. If highly experienced and reliable climbers are available they may select their own trees along a given line at the set interval of distance (Barner, 1971). This leaves the team leader free to follow and to prepare the site description.

For species which occur naturally as isolated trees, widely separated from one another in mixed forest, the problems are much greater. Little or nothing is known of the reproductive biology of most tropical high forest species. In practice the procedure followed is likely to be determined largely by the practical limitations of accessibility, time, funds and staff. Within a given area it will be desirable to collect from at least twenty trees. For species in which seed can be collected safely after it falls to the ground the problems are reduced and may even be less than in pure stands in one respect, since there can be no doubt of the parent tree. However the problem of finding trees is much greater, particularly in thick forest with climber tangles, and if climbing is necessary the carrying of equipment under such conditions, and the subsequent transport of the seed, make for slow progress. The expedition leader may decide to reduce the number of trees to about ten and to keep the seed of each tree separately, so that some estimate of variability within that population may later be possible.

Although provenance research is concerned principally with seed collection in natural forest, some trials may include collections made in plantations, for comparison. In artificial stands the collection procedures can be much simpler. Since it can be assumed that neighbouring trees are no more closely related than distant ones and, since the pattern of planting is regular, a purely random method of selecting seed trees can be easily followed. Allowing for the fact that some selection through 
thinning will probably have been done, this method might be the most suitable to compare with the wild collections. If desired, deliberate selection of superior phenotypes is also easier, since all trees in the stand have common age and history. Whatever method of selection is followed should be recorded and taken into consideration when comparisons are made with the wild populations.

\subsection{Selection of reproductive material}

It is desirable to collect approximately equal quantities of seed from each tree in a collection, so that all are equally represented. This is particularly important when the total number of trees in the collection is small. It can be difficult to follow this principle exactly when dealing with populations composed of mainly young trees, many of which may bear very little seed. To restrict the individual amount to the quantity obtainable from the least productive tree is impracticable in such a situation and, since it will be necessary to collect from a very large number of trees to obtain the quantity needed for a large international trial, some inequality in the contribution of individual trees can be accepted without risking a disproportionate representation of any one individual. However when the number of trees in the collection is less than twenty the principle of equal quantities from each should be strictly followed. If it is not, then the seed of each should be kept separately so that a combined lot with equal representation can be prepared later, if the objectives of the trial require it. (Strictly, equal representation should be based on the results of germination tests but these are rarely feasible for individual trees.)

When collecting only a proportion of the seed from a tree, it is possible to select the best, largest, healthiest fruits in the best state of maturity. This ensures the greatest yield in relation to costs of collection, transport and handling. It is also important for long term seed storage that the material should be perfectly mature and in good condition.

The need to cover a large part of a species' range during a limited season makes it necessary to start at the first site as early as possible, even though the fruits may not have reached peak maturity, to allow more time overall. Where fruit eating pests, such as squirrels or parrots, are prevalent seed collection early in the season before damage has become widespread may give much more freedom of choice of tree, and a higher total yield. It is very important therefore to recognise the earliest stage at which satisfactory seed collection can be attempted. There is a great deal of variation between species in this respect and some trial collection, starting with evidently immature fruits and proceeding with collection through all subsequent stages, may be advisable during the first season of collection. These lots must of course be kept separately, with particular care in handling and recording to ensure that they have received comparable treatment after collection and that the treatment and handing conditions are exactly known. Seed collected before peak maturity may require more careful handling and special treatment to ensure that the fruits mature and open satisfactorily. For a review of methods for recogni zing mature fruits, and for ripening immature seeds after collection, 
see Turnbull (1975).

\subsection{Techniques of collection}

For most purposes in provenance research seed is collected from standing trees, usually by climbing them. However, seed of some species can be safely collected after it falls to the ground without serious risk of damage and this method is both cheaper and quicker. Its main limitation is the uncertainty over the identity of the parent tree and the greater this uncertainty the greater is the limitation on the reliability of the information which can be obtained from research based on the seed.

A good alternative is to collect from felled trees. Providing that a large number of trees is felled over a suitable area and at a suitable time this permits the selection of individual trees to be made in accordance with the principles outlined in paragraph 2.333. In some circumstances trees may be felled specifically for seed collection, but this procedure requires exceptional justification and is normally forbidden. When collecting from the ground no special equipment is required but when dealing with felled trees a pair of secateurs may be useful.

The main requirements for climbing equipment are that it should be readily transportable and quick in operation, in addition to being safe in use. In natural forest, steep and uneven terrain, or thick vegetation may make transport of heavy or bulky equipment difficult. The need to select trees at intervals of 100 metres or more, and to restrict the quantity of seed per tree, calls for quick operation and movement. However the actual selection of the equipment will depend upon the types of tree to be climbed as well as the conditions on the ground. The simplest and most versatile aid to climbing is the steel spur or hook, fitted to the climber's boot. The design of these varies in different parts of the world where they are used but standard types are available from suppliers of forest equipment. They are used normally in conjunction with a belt or safety harness, fitted with a cable that passes round the trunk of the tree. In some areas a rope is used instead, passing round the trunk and around the body of the climber below the armpits, and tied at the side, so that the climber can brace himself against the loop of rope, while driving the foot spikes into the tree in turn. The length of the rope is sufficient to form two such loops and when a branch is met the long free end is used to form a second loop above the branch and around the climber's body, before the first loop is untied. By this means very little time is lost in negotiating branches on the way up to the crown. This method is not recommended if a tree is to be climbed several times as the spikes damage the stem; in such cases permanent pole steps are preferable.

The "Baumvelo", or Swiss Tree Bicycle, is little more trouble to transport than a pair of climbing spurs and works well and safely on tall trees with a long clear bole. It is unable to negotiate branches and is slower in action than climbing spurs, particularly on trees with low branches or those of large diameter. Both this equipment, and climbing spurs, should be used in conjunction with a safety rope, as employed in rock climbing. Additional straps and clips are available for at tachment 
to the tree. The use of the "Baumvelo" equipment is described by Seal et al. (1965) but it is no longer manufactured and difficult to obtain.

For trees one metre or more in diameter ladders may be preferable. Morandini (1961) described the main types of ladder used in climbing and their relative advantages. Conventional extending ladders of aluminium alloy may be suitable in some circumstances and if used in conjunction with climbing spurs a relatively short length may be sufficient to reach a height at which the spurs can more easily be used. One-legged ladders have advantages for use on steep and rocky ground and can be more closely fixed to the trunk, even into the base of the crown. Rope ladders are sometimes used, by throwing or fixing a light line into the crown of the tree, to draw up the ladder.

Implements for reaching and detaching fruits beyond arms' length may be simple hooked sticks or more elaborate cone rakes or pruning shears, depending on the nature of the material being collected. For trees which retain their fruits more than a year, such as pines, care is needed to avoid unnecessary damage to the next year's crop, if further collections are planned. A light tool line is used to draw up equipment for use in the crown. Light bags may also be carried to contain small quantities of seed or fruits, if they are not too large. Large fruits and also very small fruits borne in clusters, are usually allowed to fall to the ground for collection by other workers. Counting of the fruits can then be done on the ground as they are placed into sacks. Workers on the ground should wear safety helmets.

\subsection{Field records}

The field records have two main functions:-

(a) to ensure that the site can be located exactly in later years by anyone wishing to obtain more material or data

(b) to provide the information needed to interpret the results of studies and experiments based on the material collected. Some examples of forms for recording data are given in Appendix 2.2.

To locate the site, the names of territorial divisions (country, department, district, forest stand, etc.), names of natural geographical features (mountains, rivers), names of towns and villages and the distance along existing roads between them are used. Latitude and longitude should be given as exactly as possible and sketch maps showing the collection site in relation to permanent landmarks are helpful.

To assist with interpretation of results some descriptive data on the stand and its environment are needed. The number of trees included in the collection should be recorded; when 50 or more trees are included an approximate count may be acceptable. The method of selecting the trees and their main characteristics (e.g. height and diameter classes, branching habits, bole form etc.) should be noted.

The important ecological data to be recorded may vary according to 
the species and the objectives of the collection but altitude, rainfall and temperature figures from the nearest meteorological station are essential. If monthly records of rainfall, and mean monthly temperature are available these can be used to estimate the intensity of dry and wet periods. More exact estimates of the effect of limiting conditions of moisture availability and temperature can be obtained by including data on mean daily maximum temperature in the hottest months and mean daily minimum temperature in the coldest months, as well as absolute maximum and minimum temperatures recorded. Direct observations on relative humidity and moisture deficit may be included but these are rarely available in the natural forest areas where exploration and seed collections are needed. The approximate distance and compass direction from the collection site to the meteorological station supplying the climatic data should be given. Integration of site data may be facilitated by the use of climadiagrams (see Appendix 8.1).

Soil differences are rarely as important as climatic differences and it is usually sufficient to record the parent material, texture and drainage in general terms, and perhaps the $\mathrm{pH}$ of the upper layers of the profile. Notes on the main species of trees, shrubs and herbs in the association may help to reveal differences in ecological conditions between sites. They can also give clues to the influence of past interference by man (…g. forest clearance) or natural hazards (…g. hurricanes, fires, etc.) on the site.

Other data that may be of great value to future collectors are the amount of material collected, the quantity and quality of seed obtained, and the state of maturity of the fruits at the time of collection. The methods used in seed extraction and cleaning may also be of interest to other workers.

\subsection{Certification of origin}

The high costs of exploration, seed collection and field experiments call for absolute certainty of the origin of the seed. This requires very careful supervision at all stages from collection onwards, and foolproof systems of labelling the material. The procedures followed in seed extraction, cleaning, packing and transport should prevent any possibility of confusion between seed lots of different origin. If different lots have to be handled at the same place, for seed extraction for example, scrupulous attention is needed to provide physical barriers that prevent any possibility of mixing seed lots. This may mean that only one lot is handled at a time, the others being kept securely stored.

Labelling of origin is best done by allocating a serial number to each collection site, in a single series starting with number 1 . This same series can be used for all species being collected, to avoid possible confusion between parallel numbering systems. When several teams are collecting independently in the same expedition each team can be allocated a block of numbers from the same series. If seed of individual trees is to be kept apart a more complex numbering system is needed e.g. $18 / 9$, the figure 9 being the ninth tree at site number 18 . If seed from separate expeditions is being brought together in the same seed store a prefix 
might be added to identify each expedition, e.g. K $18 / 9$. Once the seed has been taken into the store it will be allocated a new accession number in addition to the one allocated in the field.

The need for certification of seed origin and genetic history was discussed by Jones and Burley (1973); the development and implementation of national and international schemes for certification of forest reproductive material are well reviewed by Barner (1974a). A scheme including rules and definitions covering most aspects of seed movement internationally has been prepared by the Organisation for Economic Cooperation and Development (OECD, 1974).

\subsection{Temporary storage and transport}

The ideal conditions to maintain maximum viability, and the ability of the seed to withstand more adverse conditions, vary greatly, not only between species but also according to the stage of maturity of the seed. Although much useful information is available for many temperate species (e.g. Holmes and Buszewicz, 1958; Wang, 1974) much less is known of many tropical species. Generally it is desirable to reduce the rate of destructive metabolism in the seed by lowering the moisture content and temperature. Drying the seed makes it lighter and easier to handle and may reduce the adverse effects of high temperatures during transit, which are mot difficult to control. Reduction in bulk and weight of the collected material greatly facilitates storage and transport and for this reason the extraction of the seeds from relatively bulky fruit is generally desirable at an early stage. A bulk quantity of seed or fruits in high temperature and humidity is very susceptible to rapid deterioration through build up of pests or diseases. These dangers may be greatly lessened by reducing the bulk of material by seed extraction and by ensuring good circulation of air. The use of insecticidal or fungicidal dusts may be advisable in some circumstances if there is a high risk of severe damage, but great care is needed in treating fresh and relatively moist seed, to avoid damage from the chemicals themselves. In general, therefore, the maintenance of maximum viability in the seed during the period after collection will depend on the ability to control the moisture content, the movement of air around the seed and, to a more limited extent, the air temperature.

The provision of an adequate supply of suitable containers for fruits and seeds is most important. Generally cloth bags are best and polyethylene bags or other plastic containers should be avoided, except for seed which must be maintained at a relatively high moisture content to survive. Sacks of coarse cloth, securely tied and labelled, may be adequate for collection from the tree, and these can be used repeatedly. Seed after extraction, or small fruits, should be placed in clean white cotton bags, for despatch, so that the identity can be marked directly on the bag, in addition to the label inside. Strong outer bags of canvas, similarly marked, should be employed and several small bags may be safely packed in one large canvas bag for transport. For some species which germinate naturally immediately on seed fall, and lose their viability very rapidly thereafter at normal temperatures, special insulated containers to control temperature and humidity in transit may be needed. 
To prevent loss of identity each container should be clearly labelled on the outside with the collection number, and should contain a label stating the species, collection number, site of collection, date of collection, weight of seed contained and name of the collector. When one seed lot is divided between two or more containers for despatch each label should state also the number of containers involved (e.g. 1 of 4 ). It may be advisable to split each seedlot into at least two parts, to travel separately, to guard against losing an entire collection through accidents en route. It may also be desirable to insure the contents against loss or

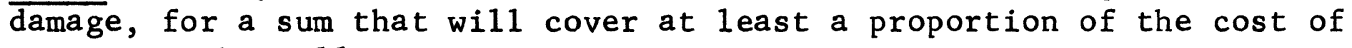
repeating the collection. 
3. SITE ASSESSMENT IN SPECIES AND PROVENANCE RESEARCH

A. Greaves and J.F. Hughes

3.1 Purpose of site assessment in species and provenance research

Since this Manual is concerned with species and provenance research, this Chapter makes recommendations about site assessment strictly in this context. It does not, for example, consider the classification of broad ecological zones, such as the system proposed by Holdridge (1967).

In species and provenance research site assessment is required for:-

(i) The description of environmental conditions, both in the natural range and at prospective planting sites. This will facilitate the selection of trial locations and the choice of potentially suitable seed sources to suit those locations.

(ii) The correlation of environmental factors with attributes of tree growth (e.g. increment, form, wood quality) of a given species or provenance, or with an order of ranking of growth attributes of two or more species or provenances. This permits the extrapolation to unplanted sites of conclusions reached from established trials or sample plots.

3.2 Proposed methods of site assessment

3.21 Assessment of the sites of seed sources

It is essential to record the conditions experienced by a population within the natural range of a species. Average values of temperature and rainfall are useful, but a knowledge of the extremes of temperature and intensity and duration of drought tolerated by a provenance are more meaningful. This is especially useful when searching for provenances suitable for use where there is a seasonal occurrence of conditions likely to place considerable stress on the trees.

Suggestions for site description of seed sources are given in Appendix 3.

3.22 Assessment of proposed planting areas as an aid to planning trials

The complete assessment of a country or region is seldom necessary. Some areas may be under a land use which precludes tree planting. Others may be unsuitable for afforestation through inaccessibility or adverse environmental conditions. Finally, forest policy may limit proposed afforestation schemes to specific areas.

It is not satisfactory to locate trials by scattering them at random throughout potential afforestation areas. Such areas are unlikely to have uniform environmental characteristics. In these circumstances the random location of trials may fail to sample a widely occurring site type.

The procedure of assessment should, therefore, be a process of progressive division and sub-division to arrive at environmental units useful 
for the planning and interpretation of trials.

Each unit should be a 'site' as defined by Coile (1952):-

"an area of land with characteristic combination of soil, topographic, climatic, and biotic factors".

The ultimate definitions of these sites will depend on the degree of variability encountered. However, a generalised procedure can be followed.

\subsection{Generalized procedure}

Classify each proposed planting area by:-

(i) Latitude - to the nearest degree. (Latitude is related to daylength.)

(ii) Rainfall - according to its distribution throughout the year e.g. uniform, one dry season, two dry seasons. If the annual rainfall shows wide variation within any category a secondary division according to mean annual rainfall will be necessary.

Draw the boundaries of proposed plantations on maps or aerial photomosaics, preferably using a scale of $1: 50,000$. On these maps, or a series of transparent overlays, mark the following, if known, in this sequence:-

(iii) Geological boundaries, or the boundaries of generalized soil groups.

(iv) The boundaries of conspicuous geomorphic features e.g. flood plains, river basins, undulating hills, escarpment $\bar{s}$, dissected plateaus.

(v) The boundaries of major topographical categories e.g. valley bottom, slope, hill crest, plateau. The definitions of these categories will depend on the degree of topographic variation encountered. Suitable categories should be determined with the aid of aerial photographs (preferably wide-angle 1 : 50,000 ) or large scale contoured maps. They should be suitable for quick identification and not refined to a degree which results in excessive fragmentation of the landscape.

The ultimate units in the classification are, therefore, units of topographic categories. These occur in groups, the number of groups depending on the environmental variability encountered during the process of the classification. Each group shares common geomorphology, rainfall regime and latitudinal range. In these circumstances the additional factors frequently cited for inclusion in site classification schemes become superfluous. Any topographic category belonging to one group will be characterized by a narrow range of altitude, soil conditions, natural vegetation type, exposure, and both macro- and micro-climatic factors. 
If information necessary for the implementation of any of the phases of this scheme is lacking that phase can be omitted and the resulting less refined level of classification accepted. Alternatively, another factor can be substituted for the recommended one. Thus maps of natural vegetation types may assist with determining the boundaries of vaguely defined topographic units or broad soil groups.

If the area under examination has had an intensive soil survey with subsequent mapping at the soil series level, then the entire classification procedure described here becomes unnecessary. A soil series can be defined as comprising similar soil profiles derived from similar parent materials under similar soil-forming conditions of climate, vegetation, relief and drainage. Recurrences of the same soil series therefore reflect recurrences of the same environmental conditions and further division becomes superfluous.

\subsection{The land system procedure}

The method described in the previous section depends on a degree of prior knowledge of the area. This is not always available and the time and resources necessary to acquire it by ground survey are prohibitively large. An alternative method is required in these circumstances.

Christian and Stewart (1952), faced with the problem of classifying large areas of land in conditions where the standard traverse method of survey was not possible, devised such a scheme.

The basis of their method is the land system. This is defined as an area, or group of areas, throughout which there is a recurring pattern of topography, soils and vegetation. A change in the pattern determines the boundary of a land system.

A land system need not be continuous. Separate examples of the same system may occur, provided that they are within the same general climatic conditions.

The main influence which determines the boundary of a land system is geomorphology. A single system has a common geomorphology throughout its occurrence. It may comprise several types of underlying geology which are no longer recognizable on the surface through overwhelming geomorphic influence, or one geological group may be divided into two or more land systems through the action of different geomorphic influences. Obviously geological material and geomorphic processes are closely related and their boundaries frequently coincide.

Since this original conception of the land system method of classification further refinement has taken place in that, not only is the land system itself described, but also the components of the recurring pattern which go to make up the land system. These components are called land facets. A land facet is defined by a combination of soil, topographic position, and vegetation characteristics. 
The implementation of the method comprises three stages:-

(i) With the aid of topographic maps and aerial photographs the distinctive combinations of landscape patterns, which constitute the different land systems, are identified and mapped.

(ii) From examinations of aerial photographs the boundaries of the land facets are mapped according to the combinations of vegetation and topography. In defining the land facet the important things to avoid are over-refinement to the point of confusion in application, and over-simplification to the point of having a unit with an unacceptably wide degree of variation.

(iii) If the necessary facilities are available a limited field survey is carried out. This usually takes the form of transects planned so as to pass through at least one example of each of the facets. This enables the characteristic soil and associated vegetation of each facet to be described in broad terms.

An example of a land system description is given in Appendix 3.1.

The scales used for the mapping of the land systems and land facets depend very much on the degree of complexity encountered. Normally land systems can be satisfactorily mapped at scales of $1: 250,000$ or 1 : 500,000. Land facets can usually be mapped at a scale of $1: 50,000$, but may need a larger scale such as $1: 25,000$ or even $1: 10,000$ in areas of great variation.

It is evident that the final unit of classification produced by the application in full of the procedure described in Section 3.221 is closely compatible with the concept of a land facet. It is useful to keep this in mind when formulating an approach for a particular set of circumstances. The land system method is ideally suited to the classification of terrain in which there is one or more distinctive recurring patterns of topography, soil and vegetation. Where there is no such distinctive pattern a compromise approach may be necessary.

Finally, choice of method will depend largely on the facilities available. The time and resources required for the implementation of a land system/land facet classification through to completion should not be under-estimated. It is not unreasonable to require a period of three years for the complete study, and presentation of results, of a square block of side $64 \mathrm{~km}$ ( 40 miles) of mixed savanna types. However, land system classifications have already been published for some regions and these provide an excellent framework for the planning and interpretation of a series of species and provenance trials (Astle et al., 1969; Bawden, 1965; Bawden and Carrol, 1968; Bawden et al., 1972; Hansel1 and Wa11, 1974; Murdoch et al., 1971; 011ier et al., $\overline{1969 ;}$; cott et a1., 1970; Wall and Hanse11, 1974).

\subsection{The distribution and location of trials}

In this Section the final unit of classification of either the 
generalized procedure or the land system procedure will be referred to by the term 'land facet'.

There will be some variation in the response of a provenance to the conditions encountered within a facet but, provided that good judgement is used in defining the boundaries of the facet, this variation should not be extreme. Consequently a provenance which is distinctly superior to all others in a trial at one location within a facet is likely to mainta in that position in replications of the same trial at other locations throughout the facet. For achieving the objectives of a species or provenance trial, i.e. determining the most suitable source of seed for a given site, an intensive network of trials is not necessary. Useful results will be obtained by allocating one trial to one example of every land facet that occurs within the proposed planting area. (An outline of the considerations in choosing experimental designs is given in Chapter 4.)

Facilities seldom exist for such comprehensive coverage, in which case priority should be given to the most widely occurring facets. The initial emphasis should be on the easily accessible sites and those which do not present large problems of plantation management.

Trials should be located at convenient points well within the boundaries of the facets. A random location of trial sites may be statistically ideal, but the sites chosen in this way may be distant from lines of access such as paths and roads. It is important to select a site to which there is easy access.

3.23 The assessment of existing plantation sites as an aid to planning sample plot work

Following the establishment of pilot plantations or commercial plantations with a chosen provenance, further investigations are required to observe how productivity varies with site or management regime. An essential preliminary to such an investigation is the implementation of a site classification scheme as described in Sections 3.221 and 3.222.

In this instance the experimental design has to take into account variability within a facet. This contrasts with the procedure for species and provenance trials. A far greater intensity of sampling is required. The actual level of intensity will be a compromise between the facilities available for the work and the size of sample necessary to achieve a desired degree of statistical accuracy.

Once an estimation has been made of the number of temporary or permanent sample plots required they should be located as follows:-

(i) Divide the number of sample plots by the number of different types of facets encountered within the chosen area of plantations (NOT the number of occurrences of all facets). This gives the number of plots to be allocated to one type of facet. 
(ii) Treating each type of facet individually, distribute the allocated number of plots amongst all occurrences of the same facet so as to achieve the same average density of sampling throughout. It follows that different types of facet may have different average sampling densities.

(iii) Locate at random the final positions of the plots within the facets.

This purposeful allocation of plots to the different units of an environment classification framework, followed by random location within the units, ensures that all environmental conditions are equally represented.

This procedure should not be confused with that followed in plantation inventory. An inventory is concerned with estimating total production on different sites. Sample plot work is concerned with estimating the magnitude of a growth response to a site, and that response is not related to the extent of that site.

\subsection{Application of the results of species and provenance trials}

The units of site description described in Sections 3.221 and 3.222 constitute areas of land "with characteristic combinations of soil, topographic, climatic, and biotic factors". Therefore, conclusions based

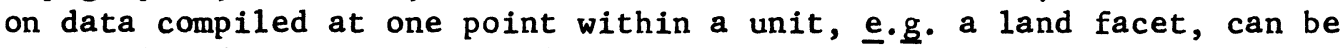
extrapolated with a reasonable degree of reliability to all other areas covered by that facet, and to other occurrences of the same facet. The conclusions from species and provenance trials will be restricted to a choice of the most suitable species to grow. The conclusions from sample plot assessments in trial plantations will be concerned with the characteristic range of growth of a certain provenance.

A distinct advantage of this system is the fact that the classification units are indirect expressions of the integration of all aspects of the environment which influence tree growth. The environmental units are identified and defined without resorting to the complex task of identifying and measuring the individual environmental factors. This approach to site assessment produces practical results which will satisfy the requirements of many organizations which are concerned with the evaluation of local resources.

3.3 Assessment of the influence of environmental factors on species and provenance growth

\subsection{Introduction}

There is also a need for a capability to predict objectively the response of certain growth attributes to a wide range of environmental conditions so that rapid preliminary assessments can be made prior to the establishment of trials. If this facility exists those species and provenances unlikely to succeed can be eliminated at the planning phase thus avoiding unnecessary work. 
The same applies to predictions of yield made from sample plot data. For this kind of work the land facet has its shortcomings, not the least of which is the division of a continuum into a number of discrete units. This is an entirely subjective operation requiring a degree of essential artistry. Some organisations with facilities for field survey and soil analysis may wish to develop more accurate growth prediction models based on the use of continuous scales, rather than discrete classes, for the measurement of environmental variables. This avoids the uncertainties associated with variability within a unit and location of boundaries.

Where organisations have these facilities, or anticipate acquiring them, a logical sequence for any site assessment study is as follows:-

(i) Classify the sites using the procedure of sections 3.221 and 3.222 .

(ii) Locate trials or sample plots as described in section 3.223.

(iii) From the assessments of these trials or plots reach conclusions on the tree growth that is characteristic of the individual environmental units.

(iv) When circumstances permit, acquire meteorological data and descriptions and measurements of environmental data by field survey. In the case of meteorological data, this is a continuous process.

(v) When sufficient data are available, investigate the feasibility of formulating a site assessment model with satisfactory predictive efficiency. It is by no means certain that a satisfactory model will be produced, and the investigator may have to fall back on the conclusions reached at stage (iii) whilst further research is undertaken. There is, therefore, a need to approach this phase with caution; the desired results may not be forthcoming.

\subsection{Difficulties in formulating site assessment models}

(i) Although considerable progress has been made over recent years in the field of plant physiology, the complex inter-actions between plant and environment are far from being fully understood. It is, therefore, impossible to predict plant growth working from fundamental cause and effect principles in the manner an engineer can forecast power unit performance from a knowledge of the design of the machine and rate of fuel input.

(ii) Those factors of the environment limiting to the growth of a given species change with location, both in identity and magnitude. Site ' $X$ ' may be inferior to site ' $Y$ ' through a decrease in the quantity of an essential factor, its total absence, or the presence of a new inhibiting factor. Consequently a list of environmental factors known to be 
limiting to the growth of a species on the first site may be completely inapplicable to the second through a change in the basic nature of the environment, and not merely quantitative changes in the magnitudes of its components.

These circumstances mean that:-

(i) It is impossible to establish a fundamental growth model suitable for practical application which relates tree growth to environmental properties.

(ii) The factors limiting tree growth in all environments cannot be described by one comprehensive set of values.

This has the following implications:-

(i) Fundamentally, models relating tree growth to environment can only be devised by means of identifying significant correlations between the two, and not from cause and effect relationships.

(ii) Environments which cannot be adequately described in terms of varying magnitudes of the same set of limiting environmental qualities have to be treated separately.

\subsection{Methodology}

Several authors have described site assessment methods, notable examples being Rennie (1963), Ralston (1964) and Jones (1969).

Those studies which have been most successful (when applied to regions of considerable environmental diversity) first eliminate variables that are non-quantifiable or difficult to measure by implementing an environmental stratification. Possible limiting factors within the units of the stratification are then measured. The data for individual environmental units are subject to a statistical screening process to identify, those variables which will serve as useful predictors of growth either singly or in combinations.

The nature of the preliminary stratification will depend on the accuracy of prediction required. For regional yield studies with the aid of sample plot data, land systems are suitable units. The land facets may be used to plan thorough coverage at the experimental design phase, but ignored in the final analysis.

For very general predictions of yield, or preliminary decisions on the most suitable provenance to plant, a more broadly based environmental unit may be adequate, $\underline{e} . \underline{g}$. one based on climatic characteristics alone.

When deciding which variables to measure within the classification framework one is faced with two possible courses of action. Firstly, from a knowledge of plant/environment relationships in general, and the known characteristics of a given tree species in particular, one can 
hazard an informed guess as to which attributes of the environment should be studied. Alternatively, one can attempt to devise a 1 ist of observations to be made with the intention of observing first, without regard to preconceived plant/environment relationships, and examining for useful trends second.

The first approach is dependent on the prior knowledge and good judgement of the investigator. The second assumes that a manageable, yet sufficiently comprehensive, 1 ist for all circumstances can be drawn up.

The first method establishes well defined guidelines from the onset, guidelines which may well not be vindicated in the final analysis. The second method attempts to preserve flexibility of approach, leaving the investigator with alternatives should his first line of analysis prove unfruitful.

It is for the second method that proposals are made here in the belief that flexibility of approach is of paramount importance.

Description of the environment

The description used here will undoubtedly be excessive for many local or regional needs. However, if site description work is to be undertaken the procedures in the following sections should be regarded as the basic objectives. These will provide a sound data base with which to meet many contingencies, whether they be the detailed information for local prediction models, or the broader generalities necessary to facilitate a meaningful exchange of data on a given species or provenance.

\subsection{Classification framework}

The procedure described in Section 3.221 is suitable for this purpose, stopping at Sub-section (iv). If a land system classification is available it should be used in preference.

Existing ecological classifications may also be used to advantage, but care must be taken in determining their relevance to plantation forestry.

\subsection{Trial site description}

Many forestry research organisations have their own systems of site description which are perfectly adequate. In view of the restricted purposes of this Manual it is inadvisable to put forward proposals which could be construed as an attempt at "standardization" in such a complex matter.

The proposals made here for site description are therefore restricted to observations that can be recorded easily, that can be related to existing and established procedures in various countries, and that are readily intelligible for purposes of exchange of information between research organizations. 
(a) Climate

For the local conditions in the vicinity of the trial a detailed description is desirable. An attempt should be made to acquire the following data, arranged in order of priority:-

(i) mean annual total of precipitation

(ii) mean monthly precipitation

(iii) mean annual temperature

(iv) mean month1y relative humidity percentage

(v) mean monthly temperature

(vi) mean daily minimum temperature of coldest month

(vii) absolute minimum temperature recorded

(viii) mean daily maximum temperature of hottest month

(ix) absolute maximum temperature recorded

(x) mean range of temperature

(xi) mean monthly wind speed at 2 metres above ground level

The number of years of observations on which each of the above categories is based should also be recorded.

It is unlikely, except for a very small minority of instances, that climatic data will have been recorded at the trial site. Some degree of extrapolation from established meteorological stations will be necessary. Where this is undertaken it is important that the vertical and horizontal separation of the two locations be noted, as well as circumstances which might indicate a difference in climatic conditions, such as a change in vegetation type, or different locations in relation to topographic features and the prevailing wind which might be the cause of differences, arising from rain shadow effects.

If it has been shown that, for the region concerned, vegetation type or altitude is a reliable indicator of climatic conditions the method can be used, but full references to the procedure should be given.

\section{(b) Topography}

The general topographic conditions will be accounted for during the preliminary environmental classification.

When the unit of classification, be it land facet or otherwise, encompasses an escarpment, or similar topographic feature, defining the trial location according to one of the categories of summit, upper slope, middle slope, bottom, will be useful. If the slope is well defined 'percentage distance from the ridge' is a more suitable scale. A similar procedure can be used when the facet comprises an undulating plateau or plain.

Situations can arise where small and gradual variations in the level of the land surface bring a water table close to the surface with consequent marked differences in tree growth. When this is suspected, attempting to account for the variation with a topographic position classification is undesirable. Owing to the slight and gradual changes the 
categories are poorly defined and excessively subjective. This condition is more easily measured from observations on the soil profile.

A topographic position is meaningless in circumstances where there is little or no altitudinal variation.

Angle of slope is an excellent measurement for describing this attribute of local topography. It is commonly expressed as slope percentage this being the natural tangent of the slope measured in degrees. The measurement should be an average reading over a transect aligned at right angles to the contour (not plot boundaries) and passing from one side of the plot to the other.

Aspect can be significant in certain conditions. This should be recorded in degrees true bearing from north. It is important that it be measured with reference to the characteristics of the unit of environmental classification. If one is dealing with a land system which is essentially a plain with only minor fluctuations in slope and altitude, which are insufficient to cause localized climatic or micro-climatic variations through modifying the influence of a prevailing wind or solar radiation, the measurements of this variable will be superfluous if not misleading when based on only the vaguest evidence of topographic variation.

This is yet another example of the necessity for determining a realistic classification framework for site description procedures, within the units of which (e.g. . land systems and facets) the description of environmental variables can be accepted or discarded depending on their relevance. The criterion for a decision of this nature is "Does the variation exist in these circumstances?"

\section{(c) Vegetation classification}

Dansereau (1951) in a critical and very thorough review of the methods of describing and recording vegetation began by stating "The geographer, the ecologist, the taxonomist, the pathologist, the geneticist and others, many others, are interested in encompassing in a classification the living beings on which they work or which presumably bear some relation to a particular organism on which they are working." He continues that there is a necessity to define "the scope of one such (method) and point to the futility of applications exceeding it".

Fosberg (1958) echoed these sentiments in stating:-

(i) that a uniform technique for the study of vegetation is neither possible or desirable;

(ii) that a natural classification of vegetation in one of the ways this term is commonly used is inherently impossible and in the others is improbably feasible, and if possible would be too complex to be readily understandable;

(iii) that no single artificial classification will likely be serviceable for all purposes, and that therefore no such classification will be generally accepted; 
(iv) that a uniform nomenclature of vegetation types, being dependent on a single accepted classification, will not be feasible.

Nevertheless Fosberg acknowledged that classifications are useful and indeed necessary, to facilitate both understanding and communication provided that their limitations are acknowledged.

In this particular instance, it is impossible to devise or select a classification scheme which will provide for all contingencies. For example, where there is diversity in vegetation appearance a physiognomic classification maybemost appropriate. Where there is only one physiognomic type, structure and composition might assume paramount importance.

In these circumstances a method of vegetation documentation is required which will enable the recovery of information at different levels of abstraction to facilitate the appraisal of different methods of classification. It would appear that the system proposed by Kuchler (1949) provides a suitable foundation for such a scheme (see Appendix 3.2).

\section{(d) Soil}

The description of the soil profile is achieved most efficiently by the completion of a tabulated checklist. A suggested format, based on the work of Jenkin (1963), is presented as Appendix 3.3.

Attempts to relate tree growth to chemical content of soils as derived from soil analyses have not been very rewarding. The more easily measured soil features such as texture and depth are generally more rewarding. It is for this reason that chemical analysis is ignored here, but it should be included if local laboratory facilities include it amongst their standardized procedures.

Again, as in previous sections, the underlying principle to this method of description is that classification of the observations should not take place during the compilation of field data. The data, if preserved in this manner, is available for reappraisal and re-classification during the analysis phase, thus preserving flexibility of method. As has been stressed repeatedly, any set of data can be handled in a number of ways depending on the objectives of the investigator, which, in turn, are dependent on the local conditions and, to a certain extent, on the background knowledge available. A soil property which appears to be significant at first sight might assume greater importance in later years when the factors limiting tree growth in general, or of a certain species in particular, become more fully understood.

This, it is hoped, will emphasize the danger in ignoring some properties of the soil profile (or of designating some as being more important than others) without very detailed reference as to the soil use (in this case growth of a species, for which the properties are being assessed).

For a full description of soil survey procedures the reader is referred to the soil survey manual by the United States Department of 
Agriculture (USDA, 1951).

3.4 Advanced descriptions of climatic conditions

Some measurements of the environment become more manageable when integrated to form indices. Climatic factors are often treated in this way. Descriptions and appraisals of these methods are given by Moreau (1938) and Jackson (1962).

Since moisture availability and temperature are overwhelmingly limiting influences on tree growth for at least part of the year in many parts of the world it is to be expected that there has been a great proliferation of climatic indices. Few, however, are suitable for general application. Those which do show promise are concerned with attempting to estimate the duration and intensity of periods of moisture excess and deficit as experienced by tree crops.

The time and facilities necessary for the computations of these indices are considerable but when results are available they provide a very meaningful means of comparing one of the most fundamental growth controlling influences at different sites. An outline of the procedures is therefore given here with the intention that they should be regarded as long term goals, not short term necessities.

A brief description of Penman and Thornthwaite's methods for the classification of climate is given together with the information that will be required if they are to be used to describe some aspects of climatic conditions in species and provenance trials. This is not intended to be an account of how to apply these methods which involve complicated techniques and require detailed study of the original references and subsequent developments. (See Thornthwaite and Mather, 1957; Penman, 1963; McCulloch, 1965). The purpose of the note is to give an outline of the assumptions on which the methods are based, the inputs that are needed and of the sort of results produced, so that forest research officers can consider the extent to which they can or should be applied in their circumstances.

\subsection{Thornthwaite's method}

Thornthwaite's method is developed from the assumption that potential evaporation and transpiration can be estimated as a function of the mean monthly air temperature, with a daylength adjustment to account for the effects of latitude and for seasonal variations, so that the system can be applied on a worldwide basis for the classification of climates.

The main advantage of the method is that it requires only records of temperature and of rainfall, which are available from nearly all climatic stations. This reduces the extent to which interpolations need to be used to estimate the potential evaporation and transpiration at a particular site. Moreover, this method does not depend on estimates from field surveys of soil moisture storage, which is continually changing and is therefore laborious to measure and difficult to define. Values for potential evaporation and transpiration are easily calculated from tables 
produced by Thornthwaite and Mather (1957) on a monthly as well as on an annual basis. The method makes use of a value for the annual heat index (I) and the mean possible monthly duration of sunlight, which can be estimated from the mean monthly temperatures and the latitude respectively, and is more sensitive than other systems based on relatively few parameters.

Thornthwaite's method, however, has some serious disadvantages. The most important is that, because of a time lag, temperature is not a good indicator of the energy available for evaporation and transpiration, which has a considerable influence on evaporation and transpiration in some places. Warm air advection may also increase air temperature, without having similar effects on solar radiation and therefore on evaporation and transpiration. Consequently, the estimated value for evapotranspiration, which is based on records of air temperature, may be considerably overestimated. On the other hand, with cold air advection, evapotranspiration may be considerably under-estimated.

\subsection{Penman's method}

Penman's method is based on the estimation of evaporation, using techniques that do not require direct measurements of surface evaporation. It has the advantage that it is based upon established physical principles and there is considerable experimental evidence that the Penman equation gives an accurate estimate of potential evaporation from open water. Tables are now available to facilitate the computation of the equation to estimate potential evaporation, and maps have been prepared for some countries in which are presented both monthly and annual potential evaporation, calculated by Penman's formula.

A serious disadvantage of the system is that it requires some information, such as the solar radiation, which is recorded only at first-order weather stations. It is therefore often necessary to estimate such parameters, either from relationships that have been established with climatic factors more easily measured, or by interpolation. This usually leads to errors in the computation of estimated values for potential evaporation $\left(\mathrm{E}_{\mathrm{O}}\right)$.

Furthermore, the Penman equation gives estimates of evaporation only from open water surfaces. The potential evapotranspiration from a land surface covered with vegetation therefore has to be estimated as a ratio of the evaporation from a water surface. This requires field trials and surveys; moreover, the relationship is not constant for all sites, and at any site it varies considerably during the course of a year and from year to year, although the latter source of variation is small for mean monthly rates.

\subsection{The water balance}

The term water balance, as used here, refers to the difference between the water received at a site (from precipitation, surface water flow or movement of ground water) and the loss of water (by evaporation, transpiration, run off and seepage). It can be regarded mainly as an 
expression of climatic aspects of the site but it is influenced also by the topography and by the soil conditions which determine maximum water holding capacity and consequently the surplus or deficit at any time. Precipitation is the only source of intake of water at a site that can be measured easily with reasonable accuracy and water balances are usually computed on the basis of receipt of water from precipitation only. This is a reasonable assumption for level sites with free soil drainage but it is clearly not applicable at sites where there is surface run off or movement of soil water. Consequently there are serious limitations to the usefulness of the method for precise description and comparison of different sites for example at the level of the Land System and Land Type.

The water balance can be computed on a daily, weekly or monthly basis. It is often computed on a monthly basis, because the required climatic data are available only as monthly averages or totals. The implication of a calculation on a monthly basis is that rainfall is evenly distributed throughout the month, and since this is not so there is an underestimation of moisture deficit or surplus. This is not likely to give rise to serious errors, and from a practical point of view it could be rather tedious to compare sites, in terms of water balance, if these were presented on a daily or even a weekly basis.

Computation of the water balance

The water balance is shown in the form of six main items:-

(a) Potential evapotranspiration (PE)

(b) Precipitation ( $\mathrm{P})$

(c) Soil moisture storage (DT)

(d) Actual evapotranspiration (AE)

(e) Moisture deficit (D)

(f) Moisture surplus (S)

An example of the computation of a water balance is included in Appendix 3.4

(a) Potential evapotranspiration (PE)

There are several ways by which this factor, which is an estimate of the loss of water that could occur at a site by evaporation from the soil and vegetation and by transpiration, can be estimated. The methods devised by Thornthwaite and by Penman are explained briefly in Appendix 3.4 and references are given above to the publications in which they are described in detail.

(b) Precipitation (P)

Values for monthly rainfall are available from most forest stations 
but this information needs to be checked carefully for errors in recording and transcription. Missing values can be estimated from the formula:-

$x_{i j}=\frac{c C j+r R i-G}{(c-1)(r-1)}$

where;

$i=$ year

$\mathrm{j}=$ month

$G=$ total of all monthly rainfa1ls

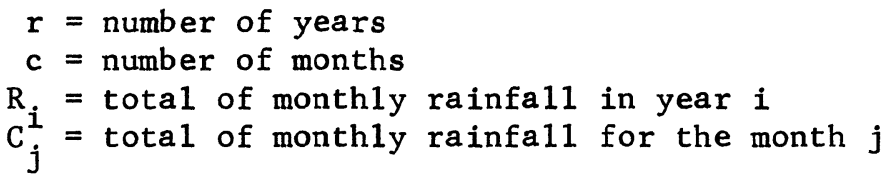

$r=$ number of years

$R_{i}=$ total of monthly rainfall in year $i$

$C_{j}=$ total of monthly rainfall for the month $j$

\section{(c) Soil moisture storage (ST)}

The maximum capacity of the soil to hold water has to be estimated for the sites that are to be described. Soil moisture storage is determined largely by soil type and structure and by rooting depth. Very often there will be no information from which the soil moisture capacity of the range of sites for a provenance trial can be calculated. It may therefore be necessary in the first instance, to use an estimated fixed value for this factor.

Differences between computed soil moisture deficiencies for soils with maximum capacities of $300 \mathrm{~mm}$ or $400 \mathrm{~mm}$ are relatively small for the months at the beginning of the dry season but they do increase during the later months of the dry season. Where the maximum soil moisture capacity is much lower than $300 \mathrm{~mm}$, and there are long dry seasons, soil moisture deficiencies, however, will be considerably underestimated when Thornthwaite's method is used with an assumed maximum moisture storage of $300 \mathrm{~mm}$. It is therefore advisable to carry out, as soon as possible, soil surveys to ascertain actual maximum soil moisture capacities in order to make best use of the methods of classification of climates and sites described here.

(d) Actual evapotranspiration (AE)

There are different opinions about the extent to which soil moisture is available for evapotranspiration over the range of soil moisture content between field capacity and wilting point. Some hydrologists maintain that the rate of evapotranspiration diminishes, slightly at first and then at a fraction of the potential rate equal to the ratio of unused available water to that present at field capacity.

The estimates for potential evaporation from open water $\left(E_{O}\right)$, derived by Penman's method, have to be corrected to obtain an estimate of actual evapotranspiration (Et) from vegetation covered surfaces. The correction factor has to be determined from field surveys and it changes as the soil becomes drier. At Mbeya in Tanzania, for example, the Et/Eo ratio was found to be 0.7 for December to May, 0.5 for June and July and 0.1 for August to November (Pereira and Hosegood, 1962; Pereira, Dagg and Hosegood, 1962). If information is not available to calculate the seasonal changes in this ratio, then the value for evaporation from open water (Eo) can be multiplied by 0.7 , a ratio commonly obtained for sites where there is sufficient water, and the product regarded as potential evapotranspiration 
by Thornthwaite's method as described in Appendix 3.4.

Thornthwaite assumed that actual evapotranspiration (AE) is equal to potential evapotranspiration ( $P E$ ) when precipitation $(P)$ is equal to or exceeds potential evapotranspiration. Where precipitation $(P)$ is less than potential evapotranspiration ( $\mathrm{PE}$ ) plus the reduction in soil moisture storage (ST), the value of which can be obtained from tables in Thornthwaite and Mather (1957).

\section{(e) Moisture deficit (D)}

The differences between the actual evapotranspiration ( $\mathrm{AE}$ ) and the potential evapotranspiration (PE) in any month is the soil moisture deficit (D) for that month. With Thornthwaite's method for computation of the water balance, the moisture deficit increases gradually during the dry season. Other hydrologists, however, maintain that a water deficit occurs only when the soil moisture storage is depleted.

\section{(f) Moisture surplus (S)}

When the soil moisture storage has reached its maximum capacity for the site any excess precipitation is counted as moisture surplus ( $S$ ) and is subject to loss by run off. In fact run off is the excess of precipitation over infiltration rather than the excess over storage. A heavy rain storm at the beginning of the wet season will therefore lead to an underestimation of the moisture surplus and consequently to an overestimation of soil moisture storage.

\subsection{Presentation of information on the water balance}

The second stage of Table 1 of Appendix 3.4 presents the essential information for the description of water intake and water loss at a site. Sites can be compared from their tabular statements and from histograms of the various parameters, including water deficit and surplus, or by preparing maps, similar to rainfall isohyet maps, in which zones of similar potential evapotranspiration (PE) or soil moisture deficit (D) are shown.

\subsection{Data analysis}

When dealing with measures of climate the inadequacies of mean values should be kept in mind. For example, in the case of mean annual rainfall the degree of variation about the mean is probably of greater relevance. It is the frequency of occurrence and intensity of exceptionally dry years that will be the ultimate growth limiting factor as far as climate is concerned in many tropical countries.

When attempting to relate growth to measures of climate it is the climatic conditions recorded during the life of the trial that should be 
used whenever possible, not the long term mean. How those conditions compare with the long term mean should then be examined. If conditions were wetter than average the conclusions reached from the trial's performance may not be completely valid. On the other hand, a species or provenance growing well when conditions were drier than average should grow as well if not better over the long term.

This example also serves to illustrate the illogicality of applying highly complex data manipulation procedures to inadequate information. The calculation of the water balance may be thought desirable but the results would be entirely fictitious if long term mean precipitation values were used for a four year old trial in a region that experienced wide year to year variation in rainfall.

When undertaking data analyses there is often the need for a realistic compromise between scientific purity and practical limitations. 
4.

PRINCIPLES OF EXPERIMENTAL DESIGN

H.L. Wright and I.A. Andrew

\subsection{Sources of variation}

Biological material is unique in its scale of variability. There may be considerable differences between populations, between plants of the same population growing in different sites and even between plants of a single population growing together. The reasons for these differences are partly genetic and partly environmental, for even plants of a single clone will differ according to the circumstances in which they are grown. The objectives of species and provenance research are to assess the differences in performance between different genotypes in a given environment and, sometimes, to observe whether those differences are the same in two or more varying environments. The major problems in fulfilling these objectives arise because:-

(a) the environment in which forest trees are grown cannot be perfectly controlled

(b) no two seedling trees of the same provenance are alike genetically, nor are they necessarily representative of their class.

In species and provenance experimentation five sources of variation may be identified. These are:-

(a) controlled genetic differences between the populations being compared

(b) controlled environmental differences within a site or between two or more experimental sites. Such differences can often be most closely controlled and measured in a greenhouse experiment.

(c) uncontrolled genetic variation among the experimental plants and between those plants and the populations they represent

(d) uncontrolled environmental variation. This includes (i) those factors of the environment which vary from plot to plot and tree to tree because of observable but uncontrollable differences in soil, microclimate, aspect, etc., and (ii) environmental differences which are not or cannot be assessed.

(e) experimental error which arises through inaccuracies of assessment and recording and through random variation within a plant, such as the difference in essential oil content between two adjacent leaves. A further and more serious source of experimental error is that of undetected mistakes in the management of an experiment, such as planting trees of the wrong provenance in a given plot.

Uncontrollable genetic and environmental variation are generally difficult to separate in experimental work; frequently it is impossible to do so and their combined effects are termed sampling variation. This in turn is often difficult to separate from experimental error; frequently 
in the literature the two have been wrongly labelled together as "error". A better term for the combination of sampling variation and experimental error is "residual variation".

\subsection{Scales of variation}

Typically, residual variation between individual experimental plots growing the same species or provenance is considerably greater than the differences between the populations. Variation between individual trees in a plot may be greater still. Thus while a difference between populations of $5 \%-10 \%$ of their mean may be of considerable practical significance, residual variation between trees and plots may be $20 \%-30 \%$ or more. The aim of experimental design is to ensure that differences between populations are not masked by uncontrollable variation.

\subsection{Models of variation}

A model of variation is implicit in an experimental design and its subsequent analysis. The model should represent the way in which an observed response (say height growth) is affected by each source of variation. For example, in a simple randomized block experiment the response may be assumed to be an additive combination of replicate (environment), population (genetic) and residual effects:-

$$
y_{i j}=m+r_{i}+p_{j}+e_{i j}
$$

where $y_{i j}$ is the observed response at the $(i j)^{\text {th }}$ plot, the mean response for the experiment, $r_{i}$ is the effect of the $i$ th replicate, $p_{j}$ is the effect of the $j^{\text {th }}$ population and $e_{i j}$ is the effect of residual variation
at the $(i j)^{\text {th }}$ plot.

Rather more complex models may be used to represent variation in other types of design; certain assumptions are, however, common because they facilitate analysis. These assumptions are:-

(a) the effects of the sources of variation are additive

(b) residuals are independent from plot to plot or tree to tree, i.e. residual variation is random

(c) residual variation is constant

(d) the residuals are normally distributed with zero mean.

The effects of departures from these assumptions have been widely discussed. In fact the common analytical methods, the analysis of variance and regression analysis, are robust, $\underline{i}$.e. they are not seriously affected by moderate departures from the assumptions. In many cases where there is serious failure of one or more of the assumptions the fault may be remedied by appropriate transformation of the data - see Section 6.132. 
4.2 Experimental design for species and provenance trials

The primary objective of an experimental design for a species or provenance trial is to ensure precise and accurate estimates of differences between populations, between distinct environments and, where appropriate, be ween silvicultural or other superimposed treatments. This may be achieved as follows. (The design chosen should be described in the control plan of each experiment - see Chapter 5).

(a) Choice of a suitable experimental site. Plots should not differ systematically across the experimental site, e.g. a soil fertility gradient. Random variation between plots and between trees should be as small as possible. However, the site should be representative of intended plantation areas to help ensure validity of experimental results.

(b) Adequate replication. Increasing the replication (the number of plots) of each population steadily reduces the residual variation associated with any comparison of population means. Theoretically the precision of such comparisons is proportional to the square root of the number of replications; in practice, however, there are other compensatory factors which reduce slightly the gain in precision. Chief among these is the fact that for any given plot size and number of populations, increased replication means a larger experiment and, consequently, a great likelihood of site heterogeneity.

There are several alternative methods of determining the number of replications required to achieve a given level of precision. (See Appendix 4 ). In order to test large numbers of populations it may be impossible to achieve replication sufficient for a given level of precision because of lack of experimental resources. Then a choice may be made between reducing the numbers of populations tested or accepting a lower level of precision. Note that precision may be improved by using a complex design such as a lattice.

(c) Control of external influences, blocking. If an experimental site were relatively uniform with only random variation from plot to plot then a completely randomized arrangement of, say, five plots (replicates) of each of 12 populations would be as efficient as any other design. Such an ideal site is rare. Field trials of forest trees cover a large area which inevitably includes systematic variations in soil, microclimate, topography, aspect, site history (í.e. previous vegetation and land use) and other factors. Even in a greenhouse there are often considerable differences in the environment from side to side, end to end, and side to centre. In the nursery there may be variation in shading, irrigation patterns, soil and exposure to wind. In all these cases the simplest method of avoiding population differences being confounded (í.e. confused) with systematic site variation is by blocking. The experimental site is divided into blocks, each corresponding to the most important environmental sub-divisions; for most types of design the blocks should be of similar size, each having the same number of experimental plots. This restriction and the frequent existence of several different sources of systematic variation mean that simple blocking rarely gives a perfect control of external influences. More complex blocking methods may be 
used to monitor two or three independent sources of systematic variation. Two types of designs, latin squares and graeco-latin squares, are particularly suitable for experiments in greenhouses and other controlled environments.

Blocks need not consist solely of physically adjacent plots; a block may be a collection of separate plots having an important environmental feature in common ( mostly contiguous, however, with some discontinuities caused by physical obstructions (e.g. roads, rock outcrops or streams) or induced by the actual shape of the experimental site.

A further advantage of blocking arises from the fact that it is often difficult or even impossible for one man, or a single team, to plant a whole trial in a single day. Similar difficulties may exist in applying other experimental treatments and in assessments. Systematic differences between operators or between days (arising because of intervening rain, say) can be confounded with block differences by ensuring that each man or team manages one block at a time.

(d) Randomization. If it is assumed that residual plot to plot variation is random there will still, by implication, be extreme plots. Some plots will be relatively fertile, for instance, and others will be relatively infertile. Assignment of each species or provenance to the plots at random is the best way to avoid bias in the estimation of population differences (through a particular population being planted on all "good" or all "bad" plots). In addition, random assignment of the population ensures an unbiased estimate of residual variation, and consequently, provides justification for the use of significance tests which are based on independent random distributions. Arguments against systematic or subjective assignment have been given by Cox (1958). (However, note that systematic designs may be useful in certain types of experimentation; see Nelder, 1962, and Section 4.235.)

Randomization should be done with the use of tables of random permutations or numbers. Comprehensive tables will be found in Fisher and Yates (1963), Cochran and Cox (1957), Green (1965) and other sets of statistical tables. An indication of how to use them is given below (4.21 and 4.22) for particular types of design. Computers are increasingly used to generate randomized experimental designs, using sequences of "pseudorandom" numbers.

If a randomization is produced which is obviously exceptional, having, say, the same or nearly the same plot sequence in several replicates, the design should be re-randomized. This advice is controversial, but has been justified by Cox (1958).

(e) Simplicity. An experiment should be as simple as possible in design, execution and analysis, as simplicity promotes accuracy by minimizing the possibility of mistakes at all stages of the experiment.

Some well known types of design, such as randomized complete blocks, are both simple and efficient (í.e. they provide a high degree of 
information for given expenditure of resources) where the number of species or provenances to be compared is small. As the number of populations increases these simpler designs often become less efficient because both random and systematic variation within replicates tend to increase. There have been two main approaches to reducing within-block heterogeneity:-

(i) to arrange plots in blocks which are smaller than a complete replicate

(ii) to reduce plot size

All these methods (randomized complete blocks, incomplete blocks and reduced plot size) are discussed below. The methods of design commonly used to increase precision in factorial experimentals such as confounding, fractional replication and split plots are not generally applicable in population trials.

\subsection{Randomized complete block designs}

The most commonly used design in forest experimentation is the randomized complete block design (RCB). The experimental site is divided into a number of blocks of equal area and each population is represented once in each block (by a plot of one to many trees). Thus each block, which should be as uniform as possible, contains a complete replication of the populations to be compared. The species or provenances are allocated at random to the plots within each block.

The sequence of operations for designing a RCB experiment might be as follows:-

(a) Determine the number of populations and the number of replications or blocks. These will be influenced partly by requirements for precision (see Appendix 4) and partly by practical limitations of resources. The number of blocks must be two or more (in order to estimate residual variation) and in practice a minimum of three is recommended and four or five blocks are often preferred.

(b) Allocate the populations at random to the plots within each block. If for example, there are four populations, the numbers $1-4$ are assigned to them in any convenient order. Their sequence in a block can then be determined from a random permutation of nine or 16 ignoring numbers higher than four. Separate randomizations should be made for each block. The result can be termed an "office layout" and may look like the plan in Figure 4.1 .

(c) Demarcate the plots in the field or nursery. Blocks should be laid down to be confounded with environmental variation within the experimental site and to take account of natural obstacles such as rivers, rock outcrops or roads. Where the site is homogeneous with only random variation, or where information on site variation is not available the plot layout may closely resemble the office layout in Figure 4.1. A more typical situation is represented by the example in Figure 4.2 where there 
Figure 4.1

A possible randomization of four populations in a randomized complete block design with four replicates

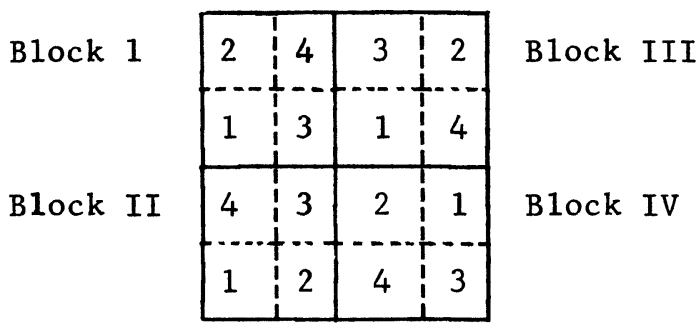

\section{Figure 4.2}

Plot layout for a field trial of four complete blocks of four populations. The site varies systematically in the direction of the arrow and there are rock outcrops as indicated.
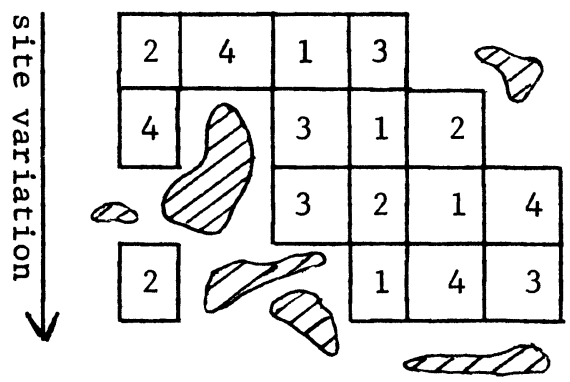

Block I

Block II

Block III

Block IV 
is systematic site variation, such as a ground slope (indicated by the arrow) and natural obstacles, in this case rock outcrops.

The size of each plot, and its layout depends upon the expected life of the trial, effects of competition between plots and management practices. These are discussed in Section 4.24 and Chapter 5.

The advantages of RCB designs are:-

(i) They are suitable for a very wide variety of experimental situations.

(ii) Analysis is simple and is easily accomplished using a desk calculator. Interpretation of results is straightforward.

(iii) They are statistically robust. There should be no problem in analysis if one or more populations fail completely or are seriously damaged.

However, the designs are less suitable where there are many populations to be compared (since the assumption of within-block uniformity becomes progressively harder to sustain) or where systematic site variation is complex. In these cases other designs should be used (see also Table 4.28).

\subsection{Incomplete block designs}

Commonly in population studies, and particularly with provenance trials, the number of populations to be compared exceeds 10 or 12 and, if there are 25-36 or more trees per plot, it is difficult to find a site with sufficient uniformity to accommodate a complete replication. Incomplete block designs have the common feature that the experimental plots are grouped into blocks of less than the total number of populations. In addition, each block has the same number of plots and each population occurs the same number of times in all (i..e. there is constant replication). The arrangement is such that variation between blocks can be estimated and eliminated from the analysis of population differences. An ideal incomplete block design is one in which each pair of populations (…g. provenances) occurs in a block together the same number of times. Such a design is said to be balanced and allows all population comparisons to be made with the same precision; however, it generally requires a large number of replications. Smaller experiments, with only partial balance are commonly used.

Incomplete block designs are not available for all numbers of populations, particularly if near or complete balance is required. However, the number of populations can often be adjusted to fit a design by either eliminating populations in which interest is low, or by including one or more additional or "dummy" populations (local, mixed collections, say). Increased replication of standard or control populations is also possible. A complete list of available incomplete block designs was given by Cochran and Cox (1957) who also described their construction and analys is in detail.

\subsection{Lattice designs}

A particular type of incomplete block design is the lattice, in which 
the blocks are physically grouped in sets which form complete replications of the populations. The number of populations must be a perfect square, $\mathrm{k}^{2}(=16,25,36$, etc. $)$, or a product of the form $k(k+1)(=12,20,30$, etc.); these designs are known as square or rectangular lattices respectively. In each case there are $k(=3,4,5$, etc.) plots per block and $k$ (square) or $k+1$ (rectangular) blocks per replicate. In general a fully balanced square lattice requires $k+1$ replicates, each arranged in a distinct way; balanced designs do not exist for certain sizes of square lattices (e.g. for $\mathrm{k}^{2}=36,100$ and 144) nor for any of the rectangular lattices.

Unrandomized plans for all the important lattices are given in Cochran and Cox (1957) and in Fisher and Yates (1963); a selection are incorporated in Appendix 5. When the required numbers of populations and replications have been determined the appropriate plan is used to produce a randomized design as follows:-

(a) If the required number of replications is less than that given in the plan select the required replications at random. Thus if four replicates of a $5 \times 5$ lattice are required select them randomly from the six given in Appendix 5, Table (d). If more replications are required than are given in the plan then two or more of the theoretical replicates must be selected and repeated. For example, if four replicates of a $6 \times 6$ lattice are required select two from the three given in Appendix 5, Table (f) and use each twice. Note that if replicates are to be repeated the total number of replications must be even. Repeated designs are rather more difficult to analyse than otherwise and any imbalance in population comparisons is magnified.

(b) Randomize the order (sequence) of the replicates actually used.

(c) Randomize the order of incomplete blocks within the replicates.

(d) Randomize the plots within each block.

(e) Assign the populations at random to the treatment numbers in the plan.

The series of randomizations described above should be carried out using a table of random numbers and permutations. The result is an office layout analogous to that described for an RCB design above (4.21). The principles for laying out of the design in the field follow those given for RCB designs; blocks should be confounded with systematic site variation and plots should avoid obvious extreme irregularities. A possible field layout of three replicates of a $3 \times 4$ rectangular lattice is illustrated in Figure 4.3; it may be compared with the plan from which it was derived in Appendix 5, Table (a).

The single advantage of lattice designs, or of any other incomplete block designs, is that the precision with which population differences may be estimated is at least as great as with a RCB design with equal replication. The disadvantages are that the designs are complex and their analyses are involved and best undertaken on an electronic computer; 


\section{Figure 4.3}

Possible field layout of three replicates of a $3 \times 4$ rectangular lattice design

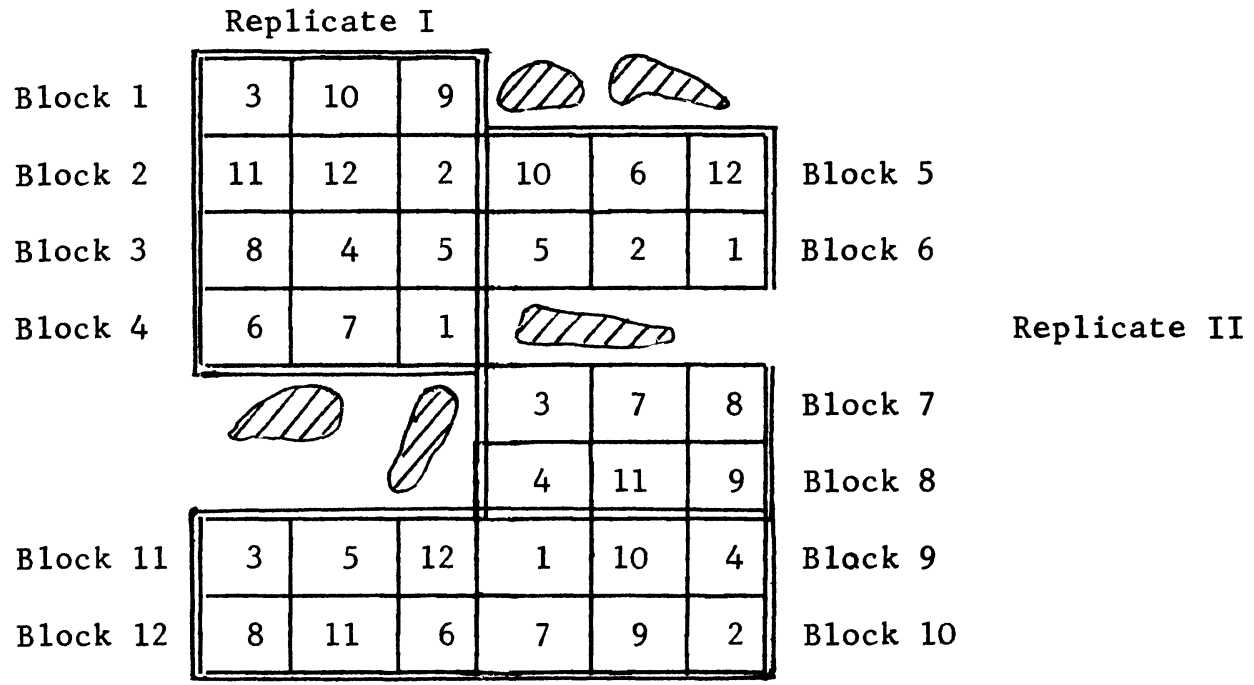

Replicate III

Figure 4.4

A latin square design for five populations

Column

\begin{tabular}{|c|c|c|c|c|c|c|}
\hline \multirow{2}{*}{\multicolumn{2}{|c|}{ Row 1}} & 1 & 2 & 3 & 4 & 5 \\
\hline & & A & C & B & $\mathrm{E}$ & D \\
\hline & 2 & B & D & A & C & $\mathrm{E}$ \\
\hline 3 & 3 & C & A & $\mathrm{E}$ & $\mathrm{D}$ & B \\
\hline s & 4 & D & $\mathrm{E}$ & C & B & $\mathrm{A}$ \\
\hline & 5 & $E$ & B & D & A & C \\
\hline
\end{tabular}


however, a lattice, unlike other incomplete block designs, is arranged in complete replicates and it can be analysed (with some loss of information) as a RCB. This feature can be of considerable utility if one or more populations fail completely, or if there are many missing values. Moreover it permits the experimenter who does not have ready access to a computer to do his own initial analyses by hand.

Cubic lattice designs exist for very large numbers of populations which form a perfect cube (é.g. $27,64,125$ ) and where site uniformity is so low that very small blocks ( 3,4 and 5 plots respectively) should be used. The use of these designs has been described by Yates (1939).

\subsection{Other types of design}

There are several other types of design which might be considered for species or provenance trials; they include fully randomized designs, non-orthogonal blocked designs, latin square, family blocks and systematic designs.

\subsection{Fully randomized design}

This is the simplest type of experimental design: individual plots of each of the populations are arranged completely at random with no blocking. The populations need not be equally replicated; there could for example be five plots of one provenance, three of another, four of a third and so on. The analysis is straightforward but the estimated residual variation may be inflated by systematic site variation; consequently comparisons between populations will be less precise than with the corresponding blocked design. Moreover, the randomization is likely to result in a disproportionate number of plots of some populations being grouped in close proximity and so comparisons among populations will be confounded with systematic site variations and will be biased accordingly. The use of systematically or randomly sited control plots (of a "standard" population, say) probably makes no improvement to precision (Cochran and Cox, 1957) and the implied adjustments to estimated population means are complicated.

\subsection{Non-orthogonal blocked designs}

This group of designs includes all those in which blocks do not contain the same set of populations. The incomplete block designs described in 4.22 are a special case but more generally each block may contain some or all of the populations being tested without there being any attempt at making balanced comparisons. A population may appear more than once in a block and there may be differential replication of the populations. Blocks may vary in size. Analysis of these designs is complicated; the principles have been described by Pearce (1965).

\subsection{Latin squares}

A latin square design enables systematic environmental variation in two directions to be estimated. The plots are arranged in rows and columns as shown, for example, in Figure 4.4. Each population occurs once in each 
row and once in each column and the number of replicates of each population is equal to the number of rows or columns. The designs are most suitable for moderate numbers of populations, as in a species trial possibly. If there are many populations a latin square is too large while for very small experiments (three or four populations) residual variation may be imprecisely estimated.

Latin square designs may be particularly useful in greenhouse experiments where the sources of systematic variation are obvious. A set of latin squares for varying numbers of populations is given by Fisher and Yates (1963). These are formal designs and should be randomized as follows:-

(a) For the appropriate number of populations select one at random from those given in Fisher and Yates (1963). (Cochran and Cox, 1957, provide one plan for each number of populations.)

(b) Arrange the columns at random.

(c) Arrange the rows at random.

(d) Assign the populations randomly to the letters A, B, C ..... used in the formal plan. tations.

Randomization should be carried out using tables of random permu-

Graeco-latin squares are designs in which three different sources of systematic environmental variation can be identified and their effects estimated. For instance, the effects of using different soils in a pot experiment could be estimated as well as the row and column effects of a latin square. The number of levels of the third factor (in the example, the number of soils) must be the same as the number of rows, columns or populations. The designs are consequently limited in their usefulness.

\subsection{Family block designs}

If the populations divide natura $11 y$ into a number of groups it may be useful to keep the groups together in blocks within each complete replication. Typically the groups may be separate species or distinct varieties, with several provenances of each. The number of provenances (i.e. group size) need not be constant. The resulting design is analogous to a split plot layout with groups corresponding to main plots and provenances to sub plots. Family block designs have been called pseudo split plot designs. The advantage of the design, in the specialized set of circumstances described, is that differences between provenances, which are likely to be smaller than those between groups (…‥ species), may be estimated more precisely.

Designing a family block layout is straightforward. The groups are assigned at random within each replication and then the individual populations are assigned randomly within each group. A possible layout for four provenances of each of three species is shown in Figure 4.5. 
Figure 4.5

A family block design for four provenances of each of three species.

Replicate I

\begin{tabular}{|c|c|c|}
\hline $\begin{array}{c}\mathrm{B}_{1} \\
(\text { Procies } 1)\end{array}$ & $\mathrm{A}_{2}$ & $\mathrm{C}_{2}$ \\
\hline $\mathrm{B}_{3}$ & $\mathrm{~A}_{4}$ & $\mathrm{C}_{1}$ \\
\hline $\mathrm{B}_{1}$ & $\mathrm{~A}_{3}$ & $\mathrm{C}_{4}$ \\
\hline $\mathrm{B}_{4}$ & $\mathrm{~A}_{1}$ & $\mathrm{C}_{3}$ \\
\hline
\end{tabular}

Replicate II

\begin{tabular}{|l|l|l|}
\hline $\mathrm{C}_{3}$ & $\mathrm{~B}_{4}$ & $\mathrm{~A}_{3}$ \\
\hline $\mathrm{C}_{1}$ & $\mathrm{~B}_{2}$ & $\mathrm{~A}_{2}$ \\
\hline $\mathrm{C}_{4}$ & $\mathrm{~B}_{3}$ & $\mathrm{~A}_{1}$ \\
\hline $\mathrm{C}_{2}$ & $\mathrm{~B}_{1}$ & $\mathrm{~A}_{4}$ \\
\hline
\end{tabular}

(For illustration only two replicates are shown) 


\subsection{Systematic designs}

Systematic designs such as the Nelder fan (Nelder, 1962) have been used or proposed for testing different species or provenances over a range of different spacings. However, these designs are not recommended for use in straightforward species or provenance trials. They have a definite use when it is desired to test a given species over a wide range of spacings and where the objective is to estimate the response of that species to increasing growing space. In this case each design should be restricted to a single species or provenance since the species-spacing interaction is of little interest.

A partial systematic arrangement of plots can be used in split-plot experiments where the effect of some factor, such as fertilizer, may be tested on a group of species or provenances. Either the main-plot treatments or the sub-plot treatments may be applied systematically. This is usually done for purposes of demonstration or where the interactions are the main object of study. For details see Cochran and Cox (1957).

\subsection{Plot size and type}

The size of experimental plots should be determined so as to provide information at the required precision for minimum cost, subject to practical limitations such as the availability of land and plant material and the need generally to grow the crop under normal management procedures.

Increasing plot size minimizes the effects of abnormal individual trees and of tree to tree microsite abnormalities. Large plots may be essential if there are substantial edge effects between plots; these will occur if, for instance, there are considerable differences in growth rate between populations, giving competitional advantage to trees of a fast growing population planted immediately adjacent to those of a slow growing one. The larger the plot size, the greater will be the number (though not the proportion) of surround trees. Surrounds are generally one or two rows deep. If the trial is to last a long time it is necessary to ensure there will be sufficient trees left in each plot to provide reliable data after one or more thinning operations. Inadequate survival, whether or not combined with the effects of thinning, may lead to some plots being inadequately represented; the analytical problem of missing plot mean values is more likely to occur with small plots.

If plot surrounds are not planted it is desirable to plant the boundaries of the trial with two or three rows of a species similar to the experimental populations. Edge effects may be considerable if boundaries are unplanted or highly variable.

Plot size should not, however, be extended beyond the requirement of ensuring that an adequate plot does exist throughout the life of the experiment. Wright (1962) has shown that the statistical efficiency of a trial decreases as the number of trees per plot increases. This is because we are generally only interested in plot mean values; the more trees there are per plot, the less is the contribution (information) provided by each individual tree. Moreover large plots mean large blocks 
for any given experimental design and so imply a greater range of residual variation within blocks. Thus within practical limits it may be preferable to use the available experimental material to provide more replications and consequently smaller plot and block size.

If a trial is not to continue beyond a thinning stage plots may be much smaller than otherwise; this is particularly so if edge effects are assumed to be minimal, as is probably the case for young trees in a trial of fairly similar provenances. The statistical validity of very small plots, including single tree plots, has been shown by Franklin (1971) not to be an important consideration. However the reliable assessment of certain characteristics such as vigour, stem form and branch habit may require larger numbers of trees to be assessed than for some other traits (Fletcher, Howe11 and Faulkner, 1968).

Tree espacement is an important determinant of plot area; $3 \times 3 \mathrm{~m}$ square spacing is frequently suggested (particularly in the tropics) since it allows easy access for mechanical weeding and reduces or delays the need for thinning, consequently prolonging the life of the trial. Assessment of height and branch and crown characteristics, and any inherent defects of stem form can be exhibited more at the relatively wide spacing.

Plot size, replication and duration generally increase through the various stages of species and provenance testing (see Chapter 5 ). Generally, however, in the later stages it is suggested that species and provenance trials are laid down with plots of $5 \times 5$ rows (․․‥ 25 trees) for assessment and a surround of one or two rows, resulting in an overall plot size of $7 \times 7$ rows ( 49 trees) or $9 \times 9$ rows ( 81 trees). At $3 \times 3 \mathrm{~m}$ spacing total plot size is 0.044 ha or 0.073 ha, respectively and the inner assessment plot is 0.0225 ha. Initial stocking is 1111 stems per hectare. There should thus be sufficient trees (twelve or more) remaining in the assessment plot for valid conclusions to be drawn at a stage halfway through a timber rotation, when stocking may be 500-600 stems per hectare. (See Appendix 11 for tables of stocking at different spacings.)

Although a square plot shape is generally recommended there are situations in which a rectangular plot may be more suitable. If planting across an incline, for instance, it may be better to plant in long, narrow plots so that systematic variation down the slope is confounded within plots. In this case the inner assessment plot may be of $2 \times 12$ trees. Note that rectangular plots are, with surrounds, rather larger than square plots with the same inner plot size.

\subsection{Layout and demarcation of the trial}

A trial should be laid down carefully and precisely according to the field plan already designed (see 4.21 and 4.22 above). Each block should be as homogeneous as possible, in soil type and depth, slope, aspect, exposure, previous land use and natural fertility.

The experimental site should be uniformly prepared according to management prescription and then the entire trial should be marked out before planting begins. (See also Chapter 5). 


\subsection{Experimental design in the nursery}

The descriptions of methods in Sections 4.21 - 4.23 referred main1y to field experimentation but generally apply equally to the nursery stage of species and provenance trials. Since the areas involved are very much smaller and the environment more controllable the designs used in the nursery may be much simpler. However, since there may be systematic differences between nursery beds it is desirable to use a blocked design and the nursery blocks should be confounded with blocks in the field design. That is, plants in any one field block should all come from the same nursery block bed. Treatment of plants within a nursery block should be as uniform as possible.

\subsection{Series of experiments}

Experimental design for a series of experiments involves no special difficulties. Each individual trial should be designed and separately randomized according to the principles already described. It may help subsequent analytical comparisons of performance if a similar design is used at each site (e.g. all lattices or all RCB) but this is not essential. The set of species or provenances planted at each site should be approximately the same to derive estimates of overall site differences. Analysis of genotype-environment interactions (see 6.135) requires as many populations as possible be planted in common at all experimental sites.

\subsection{Comparison of designs}

There are statistical techniques for comparing the cost and efficiency of various types of design; these are not considered in this Manual but some points of practical interest are listed in the following table. 
Table 4.28 A comparison of simple experimental designs

\begin{tabular}{|c|c|c|c|}
\hline $\begin{array}{l}\text { Feature of the } \\
\text { experiment }\end{array}$ & $\begin{array}{l}\text { Fully random } \\
\text { design }\end{array}$ & $\mathrm{RCB}$ & Latin square \\
\hline$\frac{\text { Number of }}{\text { replications }}$ & $\begin{array}{l}\text { Need not be the } \\
\text { same for all } \\
\text { species }\end{array}$ & $\begin{array}{l}\text { The same for } \\
\text { all species }\end{array}$ & $\begin{array}{l}\text { The same for all } \\
\text { species }\end{array}$ \\
\hline $\begin{array}{l}\frac{\text { Number of }}{\text { treatments }} \\
\frac{\text { and provenances) }}{\text { possible }}\end{array}$ & $\begin{array}{l}\text { Unlimited, } \\
\text { except that a very } \\
\text { large number may } \\
\text { lead to much } \\
\text { variability }\end{array}$ & $\begin{array}{l}\text { If too many the } \\
\text { advantage of } \\
\text { blocks may be } \\
\text { lost }\end{array}$ & $\begin{array}{l}\text { Effectively limited } \\
\text { to between } 5 \text { and } 10 \\
\text { Below this the lay- } \\
\text { out is insensitive, } \\
\text { above this, } \\
\text { unwieldy }\end{array}$ \\
\hline$\frac{\text { Laying out }}{\text { trial }}$ & Easy & $\begin{array}{l}\text { Fairly easy } \\
\text { but the blocks } \\
\text { are of fixed } \\
\text { size and must } \\
\text { be carefully } \\
\text { laid out accord- } \\
\text { ing to the site }\end{array}$ & $\begin{array}{l}\text { Design is fixed and } \\
\text { little flexibility } \\
\text { is possible }\end{array}$ \\
\hline$\frac{\text { High variability }}{\text { between plots }}$ & $\begin{array}{l}\text { No account can } \\
\text { be taken of } \\
\text { this in the }\end{array}$ & $\begin{array}{l}\text { Can take care of } \\
\text { variation in one } \\
\text { or more direc- } \\
\text { tions depending } \\
\text { on layout of the } \\
\text { blocks }\end{array}$ & $\begin{array}{l}\text { Particularly good } \\
\text { if variation is in } \\
\text { two directions }\end{array}$ \\
\hline Missing plots & $\begin{array}{l}\text { No difficulty } \\
\text { in analysis }\end{array}$ & $\begin{array}{l}\text { Little diffi- } \\
\text { culty but some } \\
\text { loss of } \\
\text { efficiency }\end{array}$ & $\begin{array}{l}\text { May entail much } \\
\text { loss of efficiency } \\
\text { and complex } \\
\text { analysis }\end{array}$ \\
\hline$\frac{\text { Residual degrees }}{\text { of freedom for }}$ & $\begin{array}{l}\text { Maximum number } \\
\text { available }\end{array}$ & $\begin{array}{l}\text { Number reduced } \\
\text { by number of } \\
\text { blocks }\end{array}$ & $\begin{array}{l}\text { Number reduced both } \\
\text { for rows and columns }\end{array}$ \\
\hline$\frac{\text { Differences in }}{\text { treatment }}$ & $\begin{array}{l}\text { Whole area must } \\
\text { be treated } \\
\text { uniformly }\end{array}$ & $\begin{array}{l}\text { Blocks may be } \\
\text { treated } \\
\text { differently }\end{array}$ & $\begin{array}{l}\text { Whole area must be } \\
\text { treated uniformly } \\
\text { if two way effect- } \\
\text { iveness is not to } \\
\text { be reduced }\end{array}$ \\
\hline
\end{tabular}


5 .

A GUIDE TO FIELD PRACTICE

J. Burley, P.J. Wood and R. Lines

\subsection{Objectives and planning}

\subsection{General considerations}

Species and provenance research should be carried out within the context of overall national forest policy - haphazard trials may sometimes by chance yield useful results, but properly planned programmes of work within the framework of national policies are essential for effective results. The research programme planned in this way aims at selecting the most suitable species or provenances, for forming well adapted, productive populations of trees in a given region.

Species and provenance trials must be aimed at reducing a very large number of possible genotype/environment combinations to relatively few proven species or provenances suitable for the production of desired forest products on relevant sites. The most suitable provenance is not necessarily that which grows fastest: in some situations the critical factor may be ability to withstand frost or drought, and at some stages of testing the ability to produce fertile seed may be important for plantation development, or for revenue from sales of seed. Other silvicultural characteristics which may be investigated may be suitability for use in windbreaks, firebreaks, erosion control and soil stabilization, and suitably for special techniques such as enrichment planting, line planting, or the special conditions of agri-silviculture in the tropics.

Provenance research also has a scientific objective, which is the definition of the genetic and environmental components of phenotypic variation. Patterns of variation in increment can be related to environmental variables in the natural habitat, throwing light on the evolution of the species. This requires much detailed work by highly trained scientists, supported by laboratory and controlled environment facilities. Such studies of biosystematics, which involve morphology, phenology and physiology should ideally precede provenance trials. They require sampling throughout the entire range of a species, examining 10-30 trees at each source, and if modern laboratory facilities are available they can provide essential information for planning provenance trials in as little as $1-5$ years. (See also Section 2.2).

Economic and social factors are not considered in detail here, but the silviculturist/ 1 must be aware of those factors which determine the range of silvicultural treatments possible within the biological range of a species or provenance. He must demand precise terms of reference for his work, where they have not already been formulated by the planners, and must ensure that staff, equipment, and funds are available to carry out the necessary programme.

I 1 The term 'silviculturist' is used here to describe the researcher, research officer, specialist, or any other person in charge of planning and implementing research. 
To compare populations empirically it is necessary to conduct experiments which are replicated, in order to minimize, or evaluate, the effects that environmental differences might have, on the apparent genetic differences between the populations. The aim is therefore to employ statistically valid designs as outlined in Chapter 4, with uniform management, for all plots. Normally, "de luxe" treatments, that is extra care in planting and tending (though not extending to irrigation) would be used, to give the plants under trial every opportunity to show their reaction to site factors not under human control. If there is any doubt that cultural practices are going to be adequate, it is better to delay expensive species and provenance trials until suitable practices have been developed. This is particularly true of nursery techniques. Species and provenance trials should not be confused with trials of cultural practices themselves.

\subsection{Objectives}

Each trial or experiment should therefore have defined objectives. This is especially true of longer term trials that may outlive their originators, and thus a clear statement of objectives is essential, $\underline{e} . \underline{g}$.

"To compare the survival, growth rate, form, wood properties and resistance to pests and diseases and any other relevant characteristics of 16 provenances of Pinus caribaea var. hondurensis on three contrasting sites in Fiji".

Amplification of these may include concise statements covering the economic implication of the species in the planting areas, and a realistic view of the products required or expected. It may be a mistake to grow a species solely for fibre, as demands for fuel or sawn timber may be critical in future markets. If possible, species should be selected which yield produce uniform in size and quality, and a general purpose tree producing poles when small, pulpwood when larger, lumber when mature, (and amenity when over-mature) may be the ideal.

\subsection{Planning and control of trials}

An idealised sequence for species and provenance trials would be as follows:-

The arboretum phase

Species elimination phase

Species testing phase

Range wide provenance phase

Species proving phase

Pilot plantations

Restricted provenance phase

Provenance proving phase

Commercial plantations

These are considered in detail in paragraph 5.15 et seq.

Control plans, or documents setting out the work to be done, are vital in any programme of research. The control plan for the individual trial (see the example in Appendix 12) may be simple but should contain certain basic information:-

(a) The objects of the trial, cross referenced to the national research programme.

(b) Proposed programme of work and completion date. 
(c) Instructions for field staff

(d) Timing and types of assessment.

(e) An outline of the experimental design and analysis

(f) Cost estimates, including maintenance costs.

The National Research Programme should include details of the project into which the trial fits. If such a programme does not exist, the silviculturist should prepare his own project programme, covering the following points:-

(a) The objectives of the project

(b) Duration of project operations

(c) Background e.g. sites to be tested, communications, land availability

(d) Overal1 proposals for management, experimental designs, assessment, analysis and publication.

(e) Staff, equipment and cost estimates

(f) Assignment of responsibility.

This plan forms the basis for the allocation of funds.

\subsection{Practical limitations}

The size of a programme of species and provenance trials depends upon many factors, including staffing, finance and the availability of land, not only for the trials, but also for subsequent planting on an operational scale. Security of tenure for the trial areas, and public cooperation in the protection of the trials themselves, are essential. The availability of suitably qualified staff will govern the kind of work that can be undertaken, and training programmes may be a necessary part of the project. The availability of transport may be critical in some countries.

The programme of operations must be worked out carefully in advance and costed as far as possible. The total cost must cover not only the initial expenses but also the essential maintenance of the plots throughout the duration of the experiments.

When staff or funds are limiting (and they are almost everywhere) the work needed must be limited in advance, and in some circumstances an inadequately replicated series of trials may be better than nothing. A precise estimate of species-site interaction, on the other hand, costs money and this must be budgeted for.

The need to 1 imit the research programme to a practical size makes it essential to locate trial plots to ensure

(a) Representative coverage of the main site types

(b) Easy accessibility for maintenance and assessment

A larger number of small plots necessitates a long total perimeter, and hence more costly protection. A high degree of replication and numerous species involves meticulous labelling and supervision which may be difficult with relatively untrained field staff. The propaganda value of a set of vigorous plots within sight of a road may be considerable and, from the practical point of view, the simpler the statistical design and layout, the better. 


\subsection{Phasing and time scales of species trials}

The "ideal" sequence of species and provenance trials is one which is often telescoped or by-passed because of the need to 1 imit the research programme for practical reasons. Nevertheless, it is vital that the duration of each trial be clearly set out at the planning phase, both for estimating at what stage the trial is expected to yield results, and for planning expenditure and manpower requirements. The duration is not solely governed by considerations of rotation length, however; it may be desired to cover the effects of the restricted rooting afforded by shallow soils on the long term growth of a species. Species and provenance research is thus a dynamic and continuing process, though it is convenient to consider it in the following phases:-

\subsection{The arboretum phase}

This often precedes the species elimination phase and has as its object the retention of living specimens of a large number of species. The number of individuals required of a species is low and the demonstration value high; a valuable features is that specimens are often kept longer than what would be considered a normal rotation. Arboreta can provide valuable guidelines for further trials.

\subsection{The species elimination phase}

Object: To compare the performance of a large number of different species on one or a number of sites, and to select a smaller number for more intensive trials.

Features: The individual species unit, or plot, is kept as small as possible and the number of species that can be tested is governed by: -

(a) Availability of funds, staff, infrastructure and seed.

(b) Ecology; for instance a deep fertile soil in a warm moist climate is potentially a more favourable site for a large number of species than a shallow acid soil in a montane region.

(c) The desired end product.

The duration of such trials is commonly 0.1 to 0.2 x rotation age, and perhaps 20 to 40 species could be tested in the initial stages, though continued introductions of small numbers of species are often made over a number of years.

\subsection{The species testing phase}

This is known also as the species performance stage (Leuchars, 1965 ) or the adaptability phase.

Object: The comparison of a restricted number of promising species, based on previous experience, on sites within a broad climatic region. 
Features: Properly designed statistical layouts are particularly important, and plots must be of a size to enable reliable assessments to be made up to, at least, the first thinning. The expected final size of the trees is important here. Sites should be stratified in order to evaluate the interaction between site variation and species differences.

The duration of these trials may be about $0.5 \mathrm{x}$ estimated rotation length, and the number of species tested in this way depends on:-

(a) Availability of funds, staff and infrastructure.

(b) The number of suitable species from previous phases and experience.

(c) Seed: it would not be normal to enter this phase unless it were expected that seed supplies would be available in adequate quantities, either from natural stands or from local seed stands.

Between 5 and 10 species is suggesed at this stage.

\subsection{The species proving phase}

Also described as management trials, and the crop performance phase (Leuchars, 1965).

Object: To confirm, under normal plantation conditions, the results shown by a small number of species that have shown themselves superior in earlier phases.

Features: Plots must be large enough to provide data on growth and yield for the full rotation surrounds must be large enough to eliminate or minimize edge effect. In addition to 'normal' plantation methods, a range of other management techniques may need to be tested, always in statistically valid designs, and including the following:-

(a) Different types of nursery stock and nursery methods.

(b) Different methods of ground preparation.

(c) Different planting methods.

(d) Spacing and thinning trials.

(e) Different weeding intensities.

(f) Pruning trials.

(g) Fertilizer and herbicide trials.

It is also appropriate to investigate wood quality at this stage.

5.155 Phasing and time scales of provenance trials

The 'ideal' sequence of provenance trials follows very closely that outlined for species above. They may be described as the Range-wide provenance phase, the Restricted provenance phase and the $\overline{\text { Provenance }}$ proving phase. Because the species involved are all 'promising', plot sizes are generally larger, and duration longer than with corresponding species trials. 


\subsection{Range-wide provenance phase}

Object: To determine the extent and pattern of variation between provenances (populations) of promising species with wide natural variation.

Features: Depending on the geographical distribution and variation of the species, 10-30 provenances are suggested at this stage. It often indicates groups of promising provenances, and also areas from which large scale seed imports should be avoided.

This phase is often run concurrently with species elimination or testing. Plot size should be small but adequate for a duration of 0.25 to $0.5 \times$ rotation age.

\subsection{Restricted provenance phase}

Object: To find sub-regions and ultimately provenances most suited to the sites under test.

Features: The differences to be detected between provenances may be relatively slight, and experimental design must take account of this. However, even within small geographic sub-regions large differences in performance may exist, and the number of provenances to be tested may not be much less than in the preceding phase. (See, for example, the international trial of Pinus kesiya described by Burley and Turnbul1, 1970). Generally, however, 3-5 provenances may be expected, with a duration in excess of $0.5 \mathrm{x}$ estimated rotation, using plots of the appropriate size. This phase is often run concurrently with species testing and species proving phases. Local land races and other derived provenances should be included where possible.

\subsection{Provenance proving phase}

At this stage one or two provenances only will have been selected for each species, site and end use. The procedure is the same as that described for species.

\subsection{Pilot plantations}

Pilot plantations of several hundred hectares are often established as an intermediate step between trials and the commencement of a large scale plantation project. Such plantations may demonstrate the viability of a planned project to potential investors and also provide an opportunity for carrying out many trials of management systems and cultural techniques. 


\subsection{Selection of sites, species and provenances}

\subsection{Selection of sites and collection of site data}

A detailed system of site classification is given in Chapter 3, and the more information available on the whole of the potential afforestation area to be tested, the more effectively can the trial programme be planned. Any site selected for trial should be examined as follows:-

(a) Is it representative of an area that is likely to be planted (not only for forest plantation but possibly also for farm woodlots)?

(b) If not, does it represent part of the climatic or edaphic range that ought to be tested?

It is also important that the sites chosen for trial cover the extremes likely to be encountered in the future, and that they include differences in exposure, frost hollows, aspect, etc.

The accumulation of information about the planting site is an essential and continuous process and should include rainfall, both past and during the currency of the trials. An important feature in dry areas is a measure of seasonal moisture availability, though practical considerations may limit the number of sites that can be investigated in this way. (See the detailed discussion in Paragraph 3.4 et seq.)

\subsection{Stratification of sites}

In the experimental statistical sense, this implies grouping areas with similar growth conditions together in a single stratum on which the performance of different species can be validly compared, and between which the site/species interaction can be determined.

\subsection{The collection of site data}

Details of a recommended method of site assessment are given in Chapter 3 and Appendix 8 and the following should be regarded as a field guide to site data collection (see also Kingston, 1974; Theron, 1973; and Toleman and Pyatt, 1974). The existing vegetation on a site is often the best indication of the site conditions over a dry period.

\subsection{Climatic data}

The following are in a suggested order of priority:-

Daily rainfall

Daily maximum and minimum temperature

Relative humidity at 0900 (or other fixed time)

Evaporation

Wind speed

Rainfall reliability. Several years'records are required for the calculation of statistical probability of a minimum annual rainfall to be received. 


\subsection{Soil types}

The examination of soil profiles over the trial site should accompany an examination of the vegetation. A pilot survey may be carried out using a small number of soil pits orientated to follow the likely direction of a catenary sequence, e.g. at right angles to the contour, or in a grid arrangement if a catenary pattern is not apparent. If large differences are found, more intensive sampling will be necessary to map the area. The depth of soil, presence of pans, differences in drainage, texture or stoniness are salient features of such surveys, which can often be carried out quickly using soil augurs, once the general pattern has been determined. After the preparation of a map showing, for instance, contours of the depth of a pan, the size and shape of the trial can be worked out. It would be necessary to ensure that no major nutrient deficiencies are likely to occur on the site before engaging in extensive and expensive species and provenance trials (see also Para. 5.432). A detailed format for the field investigation of soils is given in Appendix 3.3.

\subsection{Topography and location}

Differences of latitude are important, as they can affect day length over relatively short distances; for instance at $5^{\circ}$ latitude there is an annual variation in day length of 0.5 hour, and at $10^{\circ}$ about 1 hour. In areas of ancient soils and gentle topography, differences between the contours may be more marked than the slopes would suggest. In areas of steep topography, differences in exposure and temperature may change rapidly, and wind funnels and frost hollows may affect the location of trials. Streams and roadsides may also greatly influence the experimental layout, particularly in the location and orientation of the individual blocks or replications.

\subsection{Biotic factors}

Grazing by wild or domestic animals, or the presence of noxious weeds, such as Lantana spp., are examples of such factors that may need to be controlled. The past influence of biotic factors on a site may have modified it greatly, and the incidence of pests and diseases (such as root-rotting fungi) should be carefully examined.

\subsection{Vegetation}

The proximity of tall trees or other local variations in vegetation cover will have a direct effect on experimental design, and it is preferable to avoid major differences in vegetation even though the soils may appear to be similar, in selecting trial sites. The 'ash bed' effect from debris burned prior to planting should not be forgotten.

\subsection{The selection of species and provenances}

\subsection{Choice of product}

The technological requirements of the product are of great importance 
in deciding on species for trial, but at the same time there is a greater risk of failure in a plantation scheme if the product is too specialized. A previously valuable product may only be saleable as firewood should its market decline.

\subsection{Homoc1 imal comparisons}

The close matching of natural habitat and site for species introduction does not eliminate the need for trials, since,however accurate the formulae used, the adaptability and plasticity of a species cannot be assessed without testing. Moreover, the natural distribution of a species may be due as much to the incidence of fire, ecological competition or man's activity, as to the measurable features of climate and soil. Many species perform strikingly better in a new environment than they do in their natural habitat - Pinus radiata and Eucalyptus saligna are excellent examples of this.

On the other hand homoclimal information gained from the performance of a species as an exotic can be of much greater value, and a review of information from other countries can often reduce the number of possible species considerably and lead to the inclusion of valuable exotic land races in provenance trials.

Most obvious non-starters - $\underline{e} . \underline{g}$. Douglas fir at low altitudes at the equator - can be eliminated from the start, but in less certain cases it may be better to allow a species to eliminate itself in trial, than to eliminate it on theoretical grounds, only to have it reconsidered later when these grounds are forgotten. On the other hand, the re-testing of species that theoretically should have done well, but did not, is often worthwhile, because improved techniques or the build up of mycorrhizal populations may reverse earlier failures.

It is usually desirable to include a 'standard' well known species or provenance in trials, in order to have a reference point against which to judge the performance of the unknown populations.

\subsection{Seed}

The principles involved in seed collection from the base population have been described in Chapter 2.3. The sampling method used in obtaining seed of a species depends on its distribution, breeding mechanism and pattern of variation. Species trials may only need to include one or two typical populations from representative areas of the distribution. Provenance trials may be very different depending on whether the variation is in discrete clusters or localities (e.g. Triplochiton scleroxylon, Pinus occidentalis), widespread and uniform (many tropical pines) or linear (Eucalyptus camaldulensis).

For species with a wide and continuous latitudinal or longitudinal range, systematic sampling is desirable (after initial exploration). 


\subsection{The nursery stage in trials}

The nursery stage of a species or provenance experiment fulfills three main functions:-

(a) The provision of suitable planting stock for the field stage.

(b) Evaluation of juvenile genetic differences.

(c) Evaluation of juvenile/mature correlations and of the possibility of selecting promising populations based on juvenile characters.

\subsection{The location of nurseries}

Experience over many years in many parts of the world has shown that differences in nursery treatment can produce effects that are still noticeable in plantations many years later. It is therefore important to keep nursery treatments and conditions as uniform as possible. Nursery sites should therefore not vary much regarding light, drainage and aspect. The use of planting stock from two or more nurseries for a trial is discussed in paragraph 5.441 .

\subsection{Experimenta1 design in nurseries}

It is desirable to use replicated, randomized designs to assess the size of differences which arise from nursery practice, and it is furthermore desirable that identical experimental designs are used in nursery and in the field. In this way the two sets of replication effects will be confounded and will not reduce the precision of estimating population differences. A different situation exists where species have widely differing growth rates in the nursery, and it is less important where large differences between populations are expected later in the rotation.

Edge effects are sometimes noted with nursery beds. If growth differences are considerable, the edge plants should be discarded or used as surrounds. If edge effects appear in blocks of pots, they can be minimized by periodic rearrangement of the pots.

Poor germination may sometimes result in a shortage of planting stock of some populations. This may necessitate adjustments in the field design of the experiment. For instance, if a lattice were planned and one or two populations only were affected by poor germination, it might be best to continue with a randomized complete block experiment provided that three replications could be completed. Serious deficiencies will necessitate drawing up a fresh design for the field stage, and this should be done as far as possible under the advice of a biometrician.

\subsection{Nursery practice}

It is not possible to legislate for all types of trial and all climatic types and the following should be taken as a check list of some important points. Whatever practice is used, its features and results must always be recorded. (See Appendix 8.) 


\subsection{Germination}

(a) Seed lots should be divided, and sown one replication at a time

(b) Different species and provenances must be rigorously separated by separator boards (without causing shading) or sheets if sowing is done in a bed.

(c) Sowing should be done quickly.

(d) Pregermination, e.g. in moist sand, vermiculite or blotting paper (Granhof, 1973) may be a useful way of ensuring rapid and uniform germination, and of keeping populations separate. It also avoids pricking out at a later stage.

\subsection{2 'Pricking out' or transplanting}

If this is done, it should be done early. Plants should be pricked out when ready, regardless of differences in growth between populations. Estimates of numbers required should take account of mortality after pricking out, and of replacements required later in the field. It is at this stage that there is a particular risk of mixing populations, and no plants should be moved without adequate labels. Potted stock, which in dry or unreliable climates often give better survival, is especially prone to this.

\subsection{Culling}

The removal of very poor or deformed plants is part of normal nursery practice, but especially where 'wild' or untried seed sources are being used, a record of plants rejected should be kept, as this may be an important characteristic of some species or provenances. Normal nursery records should provide information on the number of sound planting stock produced per unit of seed sown. (See Appendix 8.1A.)

\subsection{Size of plants}

The development of nursery techniques is usually carried out concurrently with species and provenance work. If, however, markedly different growth rates are apparent in different provenances, it may not be possible to have all plants ready together, and in this case it may be better to re-sow the trial at different dates, provided seed is available, to have all stock ready at the same time.

\subsection{Mycorrhiza}

For most conifers and a number of broad leaved species, root associations with mycorrhizal fungi are necessary for healthy growth. In some countries, and especially those with mature populations of suitable species, such fungi are abundant, but where they are not, attempts to obtain useful results from trials of exotics may fail. The spread of mycorrhizal fungi may be particularly slow in monsoonal climates with a long dry season. If such problems are likely, steps should be taken as early as possible to introduce suitable mycorrhiza to the trial nurseries. Some suggested ways of achieving this are:- 
(a) Introducing suitable plants from other nurseries or areas, with mycorrhizal root systems, to nursery beds.

(b) Introducing soil from stands of suitable trees in other regions or countries (phytosanitary regulations permitting).

(c) Building up a population of mycorrhizae in the root systems of a small group of trees planted near the nursery in advance of the trials. This plantation becomes a source of mycorrhizal soil for inoculating nursery beds, and can be irrigated to achieve maximum root development.

(d) Inoculating nursery beds with mycelium or fruit bodies of likely genera, collected in the vicinity of the trials. This rather haphazard approach has proved successful in some tropical countries. The approach is less haphazard if the fungi are collected in stands of species related to those under trial. Some important mycorrhizal basidiomycetes are Boletus, Pisolithus, Russula, Thelephora.

(e) Introducing pure cultures of mycelium of fungi known to be mycorrhizal formers with the species and provenance under trial. Commercial spore cultures are not always successful outside their country of origin, and pure cultures from laboratories usually require multiplication by sub-culturing before they can be used for inoculating nursery beds.

The advice of a specialist is needed if mycorrhizal problems appear to be serious and names of workers in the field may be obtained through the IUFRO Secretariat/1.

\subsection{Priorities in nursery assessment}

The following programme of assessments is given in order of priority. Sampling intensity should ideally be related to the actual variability observed in each case, and details of how to determine the necessary sample size for each trait examined are given in Chapter 4 and Appendix 6 .

Assessments at the nursery stage are time consuming and a minimum of information should cover germination rate and percentage, age and size at planting of the seedlings. A full list of assessments indicating priorities is given in Appendix 7.1.

\subsection{Early testing and controlled environments}

The nursery stage of species and provenance trials presents opportunities for assessing differences in populations at an early stage. Similarly, trials raised in greenhouses or other controlled environments can also yield important information. There are three main objectives of this work:- Biosystematic studies, juvenile-mature correlation, and seed source identification.

/1 IUFRO Secretariat, Forstliche Bundesversuchsansta1t, A - 1131, Vienna, Schonbrunn, Austria. 


\subsection{Biosystematic studies}

The taxonomic and genecological relationships between populations are often poorly known, and the relatively uniform conditions in nurseries etc. permit more precise evaluation of genetic similarities and differences than is possible in the natural habitat, or in field experiments. Measurements are made easily, and destructive sampling is more convenient in these trials. The complex relationships between populations can therefore be studied through multivariate analysis, based on characters that are probably under more direct genetic control than are the usual field measurements of height and diameter (See also Lever and Burley, 1974).

\subsection{Juvenile-mature correlation}

If good correlations exist between characteristics at different stages of the plant's development, prediction of growth at an advanced age may be made from measurements made at an earlier stage. For instance, height at rotation age may be related to root collar diameter in the nursery (though such far-reaching correlations are rare).

\subsection{Seed source identification}

Large areas of plantations, and of trials, have been established in many parts of the world, from seed whose origin is inadequately known. It is possible to distinguish different populations by the morphological and chemical characters of seeds and seedlings, and a provenance trial is thus an opportunity to test a wide variety of populations in this way.

\subsection{Studies in controlled environments}

The use of accurately defined and controlled environments in growth chambers, phytotrons and greenhouses is particularly valuable for testing physiological responses, or the interaction of population differences with the effects of simple environmental factors such as temperature or photoperiod. Few national forest departments have suitable facilities, and research of this kind is usually carried out by specialized institutes or universities.

\subsection{The field stage}

5.41 Objects of experiments

The importance of defining clearly the objectives of experiments has been noted above. The decision on whether trials should be short term or long term depends partly on the type of trial and partly on the resources available.

\subsection{Duration of trials}

Short term trials may be defined as those in which valid comparisons are not expected to be made after major competition commences between trees, and such trials are often terminated before the first thinning. 
Little work has been published comparing the effect of different plot sizes on results obtained from a trial, but larger plots are generally required for testing characteristics in a stand as opposed to the individual tree. Single trees may on occasion be used where stand conditions are not needed, and short term trials (a few years only) are generally used to test early survival and growth, phenology and morphology, and also early resistance to pests, diseases and climatic factors such as frosts. Medium term trials may be defined as those expected to take $0.3-0.5 \mathrm{x}$ rotation length. At this stage perhaps half the final expected breast height diameter may be achieved, and these trials are generally used to investigate height and diameter increment, basal area, resistance to pests and diseases, crown form, branch and root habit, and wood properties.

Long term trials - lasting longer than half the rotation - are used to study in more detail the yield from species and provenances, and also to test the resistance to factors such as insect pests, diseases or infrequent climatic occurrences (e.g. frost). They also give information on stability, mature wood quality and mature form.

\subsection{Site selection and demarcation}

The selection of sites was referred to in paragraph 5.21 above. Administrative convenience and demonstration potential as well as site types, are important, as they affect particularly the maintenance and demarcation necessary for the trials.

Demarcation, and in particular, internal demarcation is important wherever the plot is situated, particularly when morphological differences between provenances are not obvious or not known. Plot and block corners should be marked with permanent markers, such as galvanized pipe, durable wood, or concrete posts. Embossed metal tags or large wire loops buried round the base of trees are a good system, and if there is a risk of disappearance or theft of corner posts, direction trenches are valuable. Rows of trees are easy to count when the plots are young, but they often become indistinct later, and a reliable way of finding the central assessment plot is essential. The entire experiment should be mapped in detail, with all replications and plots marked, and copies of this map should be kept in all relevant files. A notice board, particularly if it bears a sketch map of the trials, and is situated beside the route of access, is useful and may enhance public relations.

\subsection{Experimental design in the field}

The basic design of a trial depends upon its objects, but this design may be modified by the characteristics of the site. For instance, the desirability of keeping sites as uniform as possible within blocks means that their shape often has to be modified to avoid covering different soil types. Long thin blocks may best fit between contours on a slope for instance, and they certainly should not be orientated at right angles to the slope. 


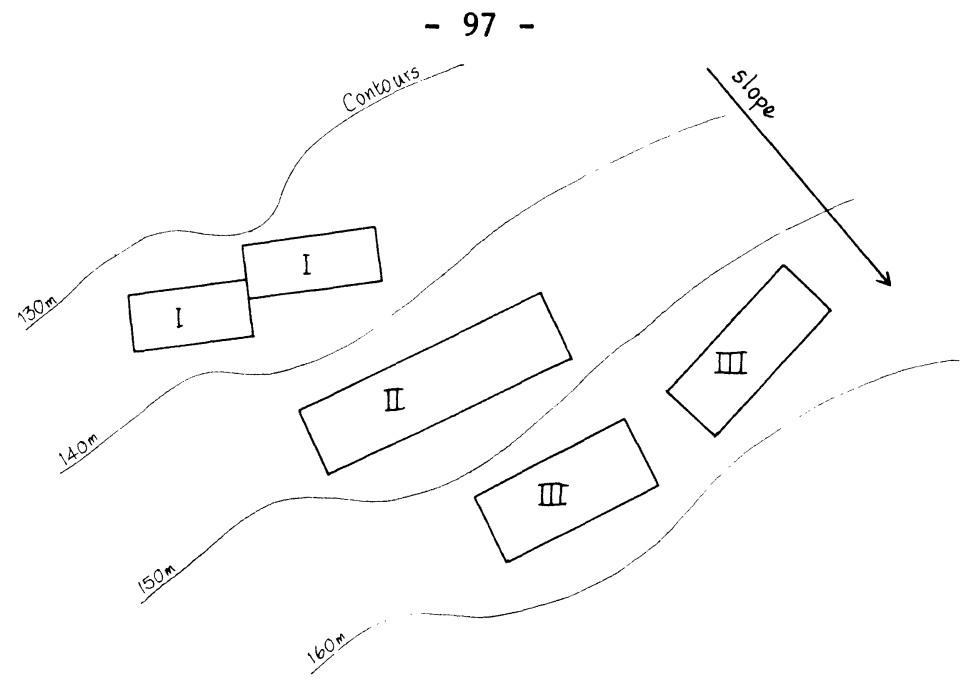

Example of Block Distribution on Hill Slope

Unavoidable variation within plots and within blocks is acceptable if this is a characteristic feature of the site, but more replications may be needed in such a case.

\subsection{Plot size, shape and competition}

The size of plots, as indicated above, depends on the duration of the trial and the expected growth rate of the trees.

As expense is also an important factor, it should be remembered that trials are grown to provide information, and that this may be obtained as well from small plots as from large. The single tree plot is of limited use except where a larger number of species or provenances is being screened for early survival, though it is cheap in space and cost, and lends itself to a high degree of replication. In the arboretum of course, only one or two specimens are required, but (assuming an initial spacing distance of 2 to 3 metres) the following numbers of trees per plot are recommended in other situations.

Species elimination phase

Minimum plot size: 5 tree line plot; Maximum: 25 trees ( $5 \times 5)$. No surrounds.

\section{Species testing phase}

Plot size $16-25$ trees ( $4 \times 4$ or $5 \times 5$ ) with a 1 or 2 row surround.

\section{Species proving phase}

Because yield estimates are important, a central plot of 100 trees ( $10 \mathrm{x}$ 10) plus a 2 row surround may be considered a minimum.

Range wide provenance phase

25 tree plots, no surrounds. 


\section{Restricted provenance phase}

25-49 tree plots, 1 or 2 row surround.

Provenance proving phase

100 tree plots (or more), 1 or 2 row surround.

Plots are usually square or rectangular, but may need to be elongated to fit certain site configurations.

\subsection{The size and effect of guard rows and surrounds}

A surround is a row or rows of trees, of the same species or provenance as the main plot, and which are not assessed. A guard row is similar, but is not necessarily of the same species or provenance as the main plot. The number of rows - or width - of surround required depends on:-

(a) The height of the trees at the termination of the experiment.

(b) The expected height differentials between plots.

(c) The degree of competition between plots.

If very large size differences are expected between provenances or species it is better to set out separate trials - for instance to separate tropical pines from eucalypts.

As a rule of thumb, at spacings of $2-3 \mathrm{~m}$, two rows of surround is often taken as a norm, but little quantitative data on the effects of surrounds have been published. For elimination trials, surrounds can safely be dispensed with.

It is usually found that there is more abundant nursery stock of some species or provenances than of others. In such cases it may be possible to use suitable surplus planting stock in surrounds to plots of those species or provenances which are in short supply.

A useful discussion on surrounds is given by Conkle (1963).

\subsection{Spacing}

A spacing of 2-3 metres has been mentioned above. The initial spacing of a trial greatly influences its pattern of growth, weed suppression, rate of spread of mycorrhizae, water requirements, and cost of planting and tending. The current management practice in the country is the best guide but, in the absence of this, a suggestion (assuming square spacing) for most species is:-

$2 \times 2$ metres:

$2.5 \times 2.5$ metres: species elimination trials: range-wide provenance phase.

species testing and proving phases; restricted and provenance proving phase. 
$2 \times 3$ metres:

$3 \times 3$ metres and above:
Unequal spacing where access for mechanical cultivation is required.

All trials involving species with a high crown/bole diameter ratio, especially tropical hardwoods.

Even in temperate regions it should seldom be necessary to plant more densely than $2 \times 2$ metres. A table of stocking per hectare for various spacings is given in Appendix 11.

\subsection{Repetition of experiments in time}

Plots planted in different years from the same seed source often show marked differences in growth at the same age, that are not attributable to site differences. This may be due to a number of factors, including nursery and planting efficiency, differences in maintenance standards and differences in climate. These factors together make up the effect of different years.

Repetition of a trial in successive years may be necessary if annual climatic variation is considerable; large differences in the performance of drought tolerant and drought susceptible species have often been found between wet and dry years.

Climatic factors cannot be randomly replicated, and therefore it is not strictly possible to 'replicate' in time as well as on different areas of land. However, trials in which both time and space are tested in this way have been laid down, and have been called SPRATS (Species trials Replicated in Area and Time; see Leuchars, 1965). Such trials involve the planting of plots of widely different ages next to each other, and difficulties oftenarise, such as the non-availability of the same species or provenances throughout the time scale of the experiment. It is generally simpler and cheaper to plant individual, discrete, trials in separate years. Moreover, if first and second year survival are the main parameters to be studied, a series of short term trials to test these would be a more effective use of resources.

\subsection{Site preparation and fencing}

\subsection{Ground preparation}

Ground and site preparation vary considerably between different parts of the world, but the effect of ground preparation treatments can be very marked and last throughout the life of the crop. An examination of the site and soil profiles may give an indication of what is required, if techniques from existing plantation schemes cannot be applied. If ripping or ploughing is needed, such work cannot usually be carried out by hand, and the logistics of organising the availability of machines over a number of scattered trial sites will have to be carefully arranged well in advance.

'De luxe' treatment, that is measures to combat local hazards, such as intensive weeding or the use of insecticides against leaf cutting ants or termites, should be used 
in order to reduce as far as possible the chance of cultural methods masking the effects of other site characteristics. Generally, therefore, competing vegetation should be reduced to a minimum and the type of planting stock and method of planting should be the optimum for the area concerned. For instance the digging of adequate-sized pits for ball rooted stock may be necessary, whereas crowbar planting is all that is needed for stumped planting stock of Tectona grandis L.f.

Species and provenance trials should not be confused with ground preparation trials, which should be carried out as separate experiments.

\subsection{Fertilizing}

If fertilizing is standard practice it should be carried out over the whole trial area but, if there is some doubt about it, two approaches to fertilization are recommended:-

(a) Fertilizer is applied to a single replication or block only, or to an extra block planted for this purpose. This will give an indication of differential response between species or provenances, but in that block only.

(b) If the site is highly variable it may be better to use a split-plot design, applying fertilizer to one half of each plot. This either reduces the effective size of the plots, or necessitates an increase in their size and hence of the experiment.

\subsection{Fencing}

Since many trials are in areas where few forestry activities are already in progress, fencing may be essential. Deep ditches and heavy fences may be necessary to exclude elephants in some areas, whereas in others, multi-strand barbed wire or wire mesh may be needed to exclude goats, voles or rabbits. The potential value of the information to be gained from properly conducted trials is high, and money spent on fencing is rarely wasted. Moreover, the materials can often be re-used at the termination of the trial. High tensile steel stock fences may not be a luxury in areas where stock damage is serious, and estimates for such protection should always be included in financial provisions for trials, and the necessary standards laid down in the experimental plan.

It should be remembered that both the size and shape of plots affect the length of perimeter fence required and small scattered experimental sites are particularly extravagant in fencing. These are also the ones that are most likely to need the protection of well maintained fences.

\subsection{Pre-planting activities}

\subsection{Transport of planting stock to the field}

If the plants are raised in a single nursery, it ensures that they have all had the same basic treatment. The disadvantages may be that:- 
(a) Stock has to be transported long distances.

(b) Climatic or other damage may destroy the whole experiment.

(c) Planting time may not coincide everywhere with the time for lifting plants in the nursery.

However, raising plants at two or more nurseries produces different types of stock that should not be used interchangeably. Therefore, the numbers of plants and the transport arrangements for each site must be worked out well in advance. Four wheel drive vehicles will be required in some areas; in all areas, physical damage to stems and roots must be avoided and, throughout, extreme care must be taken to avoid excessive transpiration resulting from exposure to wind or loss of soil from the roots. There should be a minimum of delay between delivery and planting of plants; they must be carefully labelled at all times, and if possible each nursery replication should be transported and planted together.

\subsection{Identification of planting spots and individual trees}

The dangers of loss of identity in the field in a large experiment cannot be overemphasized. Each planting spot should be marked, e.g. with a coloured stick or other clear identification. The numbering of individual trees is not usually done in early-stage trials, but if required in proving phase trials, trees can be conveniently marked with metal tags on aluminium or copper nails, or on large wire loops buried round the base of the tree, as for corner markers. With slow-growing species, the use of numbers painted directly on to the bark maybe possible.

\subsection{Planting and replacements}

5.451 Planting

Uniformity of the quality of planting should be aimed at, as variations in planting skill can have a significant effect on survival and early growth. Great care is necessary to avoid mixing nursery stock, and no plants should be removed from the nursery without clear labels. Before planting, plants should be placed at their appropriate planting spot and re-checked. In some countries it is customary to grade nursery stock and if desired, this can be done with experimental material by placing different grades of planting material in different blocks or replications, but it is generally preferable to confound the identity of nursery and field replications instead.

Speed of planting is essential to minimize the effects of day-today variations in weather, and as far as possible the same team of workers should plant a complete block at a time. If, for instance, $1 \frac{1}{2}$ blocks can be planted in a day, it may be better to reduce the target and complete a single block per day, thus confining the differences due to daily weather within the blocks.

\subsection{Replacements}

Beating up, or the replacement of casualties is an essential part 
of plantation practice and must therefore have some place in species and provenance trials.

In elimination trials care is needed to see that serious mortality is not masked by unrecorded replacements, and a sound policy is to make no replacements at all, unless obviously due to bad planting or to an accident. However, the small plots recommended for elimination trials should make extra careful planting easier; the survivals assessed can then be used with greater confidence.

In testing and proving trials it may be assumed that the species and provenances have already been tested for early survival, and replacements one and even two years after planting may be permitted, especially if it assists canopy closure. A contrasting species or provenance can be used for this practice to be removed later, as thinnings. The date and number of replacements should be carefully recorded, and if their location is known it is possible, though tedious, to undertake separate analysis for them.

\subsection{Maintenance and protection}

\subsection{Weeding}

The effects of different intensities of weeding on growth and survival can be as marked as those of nursery and ground preparation methods. Weeding should be carried out as quickly as possible, and if there are delays, one block should be weeded at a time, to maintain the conditions within each block as uniform as possible. Mechanical weeding can be carried out more rapidly, and this requires previous planning of the spacing used, to allow room for tractors and implements. Weeding should be carried out on the basis of regular inspections, unless there is an existing standard programme for the weeding of plantations in the area.

Criticisms are often made that species and provenance trials rarely give any information on costs of various operations. The information which these trials are designed to provide, however, is the response of a genotype to a certain set of site conditions. A record of costs, though useful, cannot be applied to large scale plantations without further trials of the maintenance operations themselves, to determine their effects on the productivity of the very few species whose suitability for the site has already been determined.

\subsection{Thinning}

Thinning becomes necessary at certain stages in the development of competition within a plot, although there is a good case for leaving trials unthinned, in order to determine at what stage basal area becomes limiting for further increment. Thinnings should always be carried out to have the minimum effect on the comparison between species or provenances. After thinning, the comparative information becomes less reliable, and as a general rule it is suggested that testing and proving phase trials should not be thinned until no longer required for 
experimental measurements. In many commercial situations early thinnings will be unsaleable.

If it is decided to thin trials (and thinning can provide useful preliminary information for volume tables, or on wood quality) there are two possible situations:-

(a) Where some information on the species, or a related one is available, either from the area under trial or from another country. Some idea of a specified basal area and stocking can be obtained, depending on growth rate, though at best this can only be a guide.

(b) Where no information on the species or provenance is available. Carrying out thinning by eye can lead to over-thinning in heavycrowned species, and under-thinning in those with thin crowns. Crown thinning is preferable to 'low' thinning, and it is recommended that it is carried out to free the crowns as far as possible. In any species, an estimate of crown/bole diameter ratio may be obtained from free growing specimens, and this ratio and the mean diameter of the plot may be used as an indication of stocking required.

In many species and provenances collected from 'wild' sources, the variability between trees in plots is considerable, and thinning in such plots should be designed to bring out the best in the population, rather than to maintain the full range of desirable and undesirable stems. In all cases a careful record of number of stems, basal area and volume, removed in thinnings, and left in the plot, must be kept.

\subsection{Pruning}

The degree of self pruning that a species achieves is always important and should be recorded. In all species it is desirable to prune to head height for access, maintenance and protection. Whether higher pruning is justified wil1 depend on whether it is desired to demonstrate bole form more easily, or whether more information on branch persistence is needed.

\subsection{Protection from grazing}

Fences, as described above, are both physical and psychological barriers, and are vital to protection from wild and domestic animals. Frequent patrols to remote plots for fence inspection and maintenance are usually necessary, and the siting of plots for easy access for this purpose also has considerable importance.

\subsection{Fire}

In many countries, fire is the greatest risk to trial plots, which are particularly vulnerable when young. Small and scattered plots are often difficult to keep under continual observation and there are three main approaches to fire protection:- 
(a) Reducing the amount of combustible material in the plots (early burning).

(b) Preventing fire reaching the trials, or spreading between plots (fire lines).

(c) Providing fire fighting materials.

In areas where there is a rapid growth of herbaceous material at the end of the wet season which dries to become a fire hazard in the dry season, burning before the vegetation is completely dry can effectively prevent disastrous fires. The timing of the burn is of great importance as it depends on the moisture content of the combustible material and on the weather conditions, but under suitable conditions a "cool" burn which does not damage the trees can be achieved.

One of the most effective ways of preventing fire entering a plot is the fire line. A clean trace, hoed by hand, or by harrowing, is effective, and a system of double traces, between which the vegetation is burned off, is also used in some places. Planting a different species between or round plots, either to act as spark arresters, or to leave a clean floor free of vegetation has been used in some countries. For either purpose species which grow very vigorously are required (e.g. Eucalyptus spp.). However, species of Eucalyptus are often so competitive that they affect the growth of the populations under trial, and, as their effectiveness as spark arresters is doubtful, they are generally no longer used.

In regions where fire is a hazard at some time in the year, each trial site should be provided with simple fire-fighting tools, such as fire beaters. Trials within range of a forest station or fire post will be less liable to damage than those which are more remote. Where incendiarism, for whatever reason, is a possibility, good public relations will be of the greatest importance and the public will often have to be relied on for voluntary help in fire fighting.

\subsection{Priorities in field assessment}

Assessment is time-consuming and expensive; therefore the characters to be assessed, and the timing of assessments should be laid down in the control plan.

The initial calibrators of the field stage are assessment of height a few weeks after planting (to allow the soil to settle) and a survival count.

The characters that can be assessed subsequently are as follows:-

Growth: Dry matter production/volume of stem

Morphology:

Seedling: shoot/root ratio

Stem: lean, bow, crookedness, persistence, forking, taper, circularity, buttressing, fluting 
Crown: length, diameter, shape

Bark: thickness, texture, colour

Branch: number, diameter, length, angle, type, self-pruning

Shoot: colour, texture, indumentum

Leaf: length, width, colour, form

Fruit: dimensions, form

Physiology

Phototropism, phenology, photosynthetic rate, respiration rate, flowering, lammas shoots.

Robustness and health: resistance to pests, diseases, other factors (e.. . fire, drought).

Technological: Wood properties, wood anatomy. Cellulose production and pulping properties.

Chemica1: Nutrient uptake, foliar analysis, formation of chemical compounds (e.g. oleoresins).

Clear priorities are required for the work that can be carried out in any trial. An outline of these is given in Chapter 6 , and the following are notes on the most important.

\begin{tabular}{|c|c|c|}
\hline Character & Frequency/stage & Method \\
\hline Hea 1 th & continuous & $\begin{array}{l}\text { Note incidence of pests and } \\
\text { diseases. Identify pests and } \\
\text { pathogens. }\end{array}$ \\
\hline Survival & $\begin{array}{l}1 \text { yr. old. } \\
\text { Subsequently } \\
\text { after climatic } \\
\text { extremes, etc. }\end{array}$ & $\begin{array}{l}100 \% \text { count. } \\
\text { Sample count. }\end{array}$ \\
\hline Mean height $(\overline{\mathrm{h}})$ & $\begin{array}{l}\text { Annual up to } \\
\text { about } 7 \mathrm{~m} \text { height, } \\
\text { Then every } 2-5 \\
\text { years }\end{array}$ & $\begin{array}{l}\text { Use measuring poles up to } 7 \mathrm{~m} \\
\text { then by optical instruments } \\
\text { Accuracy: aim at } 5 \% \\
100 \% \text { in initial stages, or } \\
\text { sample. }\end{array}$ \\
\hline $\begin{array}{l}\text { Dominant height } \\
\left(\bar{h}_{\mathrm{dom}}\right) \text { (mean height } \\
\text { of } 100 \text { trees of } \\
\text { largest diameter per } \\
\text { ha) }\end{array}$ & $\begin{array}{l}\text { Annual up to } 7 \mathrm{~m} \\
\text { height. Then } \\
\text { every } 3-5 \text { years }\end{array}$ & $\begin{array}{l}\text { As above, but less time con- } \\
\text { suming. } \\
\text { Accuracy: as above. }\end{array}$ \\
\hline
\end{tabular}




\begin{tabular}{lll}
\hline Character & Frequency/stage & Method \\
\hline $\begin{array}{l}\text { Mean diameter }(\overline{\mathrm{d}}) \\
\text { at breast height }\end{array}$ & $\begin{array}{l}1.3 \mathrm{~m} \text { from } \\
\text { ground. } \\
\text { Annually after } \\
\text { crop is 2-3 m } \\
\text { high }\end{array}$ & $\begin{array}{l}\text { Diameter tapes most convenient, } \\
\text { or callipers. 100\% sample } \\
\text { essential } \\
\text { Accuracy: aim at 1-2 mm }\end{array}$ \\
\hline Basal area & & Obtained from measurements of d \\
\hline $\begin{array}{l}\text { Stem form } \\
\text { Branch size and }\end{array}$ & $\begin{array}{l}\text { Start when trees } \\
\text { are } 7 \mathrm{~m} \text { tal1. } \\
\text { Then at 3-5 } \\
\text { year intervals }\end{array}$ & $\begin{array}{l}\text { Simple realistic systems best, } \\
\text { using scoring, 1-7 basis. }\end{array}$ \\
\hline Bark thickness & $\begin{array}{l}\text { Whenever d is } \\
\text { measured and on } \\
\text { thinnings }\end{array}$ & $\begin{array}{l}\text { Bark gauge, or bark removal } \\
\text { on thinnings; sample 5-10\% } \\
\text { of trees }\end{array}$ \\
\hline
\end{tabular}

Stem form is often best based on a theoretical utilization category (see e.g. Barrett and Mullin, 1968) and the number or percentage of trees in each category assessed. For stem form a suggested system using numerical scores is:

FORM CLASS

1 straight: meets or exceeds transmission pole specification 2 nearly straight: meets any but the most exacting requirements

3 very slightly crooked: adequate for most purposes but not transmission poles

$4 \quad$ slightly crooked: some value as a long pole

5 crooked: practically no value as a long pole

6 very crooked: suitable only for short poles or fuel

7 malformed: suitable only for fuel

The object of using 'scoring' systems is to produce figures that can be analysed statistically. However, the scores themselves should not be used for analysis, and the numbers of trees in each category used instead.

\section{$5.48 \quad$ Recording systems}

A clear and accurate data recording system is essential for all experimental work. Each item of data must be clearly identified and errors in recording and transcription occur all too easily. Most commonly, data are recorded by hand on specially designed forms, and these should be simple to use and provide a permanent record. Examples of these forms are given in Appendix 8. Other methods for recording and storing data are given in Chapter 6 . Whichevermethod is used, the data should be 
analysed as soon as possible, as the results, besides being of interest in themselves, may affect the future management of the trial.

\subsection{Use of results}

A detailed discussion of the types of analysis suitable for species and provenance trials is given in Chapter 6, and an indication of how it is expected the results should be analysed should be given in the experimental plan. The aim of the trials is to provide information on which management decisions can be based, often when the trials are less than half rotation age. However, the results from early stage trials are often used as a basis for further trials, rather than for management purposes.

Results from species elimination trials are used to select species for the testing and proving phases. Similarly, results from the rangewide provenance phases are used for more intensive testing from a more limited area. In the proving phases and in pilot plantations, the species used should be based on valid and accurate preceding work. The final selection of species or provenances should be based not only on the results of trials but also on the price and availability of seed.

The results and analyses of data should first of all be made known to the executive head of the forest service, to enable important management decisions to be taken. Summary reports produced periodically are often best made in the form of tables, which may also assist in prompt publication. Data prepared in a computer-compatible form (see the INTFORPROV system for instance, Burley, Andrew and Templeman, 1973) is most convenient and it is hoped that standardisation and presentation of results, especially from internationally organised trials, will increase.

Agreement on the responsibility for authorship, and setting a reasonable date for publication, beyond which publication responsibilities should be reliquished to an alternate author, is important. It is also desirable that all workers should be able to review critically manuscripts of reports on those trials that they have been involved in. 


\subsection{Assessment}

Assessment of trials is often lengthy and expensive and only those characters which are practically important and in which there is substantial experimental variation should be assessed. Quantitative traits are generally better than qualitative ones for subsequent analysis and interpretation and are often easier to assess objectively.

A measuring device should be appropriate to the trait to be assessed, to the conditions under which it is used and to the required precision of assessment. For example, measuring rods with one or two centimetre precision may be necessary in order to identify small differences among young trees; with large stems, however, where small differences have little practical significance, the convenience of a clinometer or similar optical device is much more suitable. Instruments should be used correctly in order to avoid bias or unnecessarily inaccurate observations. Assessment and recording should not exceed the real precision of an instrument (by, for example, unreliable interpolation).

Some of the traits which should be assessed at various stages during a species or provenance trial are discussed below. The lists do not include biochemical characteristics, site assessment, nor wood properties which are discussed in Chapters 2, 3 and 6.3 respectively. More details of the type, frequency and precision of assessments in comparative trials are given in Chapter 5.

\subsection{Seedling and nursery stage}

Plant characteristics in the nursery should be assessed as they may help to explain variation at a much later stage. Thus early vigour may be correlated with survival or with ultimate growth in the field. It is generally impractical to label plants individually in the nursery and 80 it is impossible to compare nursery and field performance on a tree by tree basis. This is unimportant insofar as interest is mainly centred on population and environmental differences. Assessments of height and diameter in the nursery are conveniently made on samples of 25-50 trees per population per block.

The following traits should be assessed if possible:-

(a) Average seed weight (as 1000-seed weight) and size

(b) Median (or arithmetic mean) germination time, assessed as numbers of plants germinating day by day.

(c) Percentage germinated within stated period of time.

(d) Seedling height, measured to the nearest millimetre. Depending on the species this may be measured to the top of the shoot apex, apical bud, tip of tallest leaf or cotyledon, etc. Hypocotyl and average cotyledon length should be distinguished.

(e) Number of cotyledons (for coniferous species).

(f) Nursery height, measured to the nearest centimetre, just before planting. 
(g) Nursery diameter, measured to the nearest millimetre (with a suitable pair of calipers or a step gauge at the base of the plant or above the root collar if prominent. Diameter should be assessed at the same time as nursery height.

block.

(h) Nursery survival, assessed on 100 plants per population per

\subsection{Field stage}

Assessments in the field are generally made on all the trees, or at least those in the inner assessment area of each plot. The assessment of some characteristics, such as survival, may be based on the entire plot, including surrounds, if it is believed that edge effects are not important. Other assessments may be made on the whole plot in order to estimate the effects of competition between plots. Preliminary sampling and analysis will, however, indicate if it is necssary to measure all trees to obtain acceptable precision.

Since data are collected tree by tree it is possible to investigate relationships between juvenile and mature characteristics of individual trees.

Although the range of traits assessed in the field will vary somewhat from country to country and will also depend partly on the species being grown and its intended use, the following 1 ist will be common to most trials:-

(a) Height, measured immediately after planting, at the end of the first growing season (or after a year if growth is more or less continuous), then once a year until canopy closes and subsequently progressively less frequently. Height should be measured immediately after major operations such as thinning or pruning. In the early stages of the trial height should be measured with a rule or with rods, to the nearest completed 1-, 2-, 5- or 10-centimetre class as appropriate. Later, when average height exceeds about seven metres, an optical device should be used.

(b) Diameter at breast height (d) should be assessed at the same time as height, once the crop exceeds $2-3 \mathrm{~m}$ height. It is most easily and accurately assessed with a diameter tape and provides, especially in the later stages of the trial, the most reliable single indicator of usable tree volume. Fibreglass tapes do not corrode nor stretch appreciably.

(c) Survival, assessed as a proportion of the trees planted per plot. Survival should be assessed at the end of the first growing season and again a year or so later. Subsequent assessments should be made following severe or unusual climatic events (such as frost, drought, flooding or gales) or if the trial is affected by any pathological disorder. In these cases a more comprehensive assessment of degree of damage might be made; an artificial scale of at least five classes, ranging from no damage to most severe (fatal) damage, should be used.

(d) Stem form assessed in one of the following ways:- 
(i) on an artificial class interval scale ranging from say, 0 = quite straight, through 3 = moderately sinuous, to 6 = firewood, with appropriate intermediate classes;

(ii) as the longest length of usable log which could be extracted (this will be partly dependent on the intended end use; see e.g. Barrett and Mullin, 1968);

(iii) by means of a mechanical device for measuring angle of lean, degree of bow, etc. (see e.g. Hans, 1972).

If possible stem form should be measured fairly frequently and from an early stage as it is likely that early faults are perpetuated.

(e) Crown characteristics, including crown length and diameter. These can only be realistically assessed before canopy closure; beyond that there are effects of competition and the practical difficulty of distinguishing trees. Crown size may be related to growth and to wood properties.

(f) Branch habit, including branch length, branch diameter, branch angle, number of branches per node (for whorled branches) or per unit of stem length, and, particularly where growth is not uninodal per year, inter-nodal length. Branch length, diameter and number are most conveniently assessed on a node selected at a given point e.g. at $30 \%$ or $50 \%$ of tree height or crown depth. Branch characteristics need only be assessed once or twice in the life of a trial.

\subsection{Data recording}

It is essential in all experimental work to have a clear and accurate system for recording data. This is especially true for long term forest experiments such as species and provenance trials in which large amounts of data are collected, analysed and compared over a long period of time and by many people. The most important requirements of a recording system are as follows:- each item of data collected must be clearly identified; errors in recording and in transcription should be avoided; the system should be practical and convenient to use in the field and should allow the data to be extracted easily for subsequent analysis. (See Andrew and Burley, 1973.)

Most commonly data are recorded on carefully designed, special forms. These have the advantages of simplicity, cheapness, permanence and adaptability to a wide range of circumstances and types of data. Examples are given in Appendix 8. Measurements can be checked very easily in the field, or later, and apparent errors identified. However, manually recorded forms have the disadvantage, if statistical analys is and permanent archiving is done by computer, that the data must be transcribed to a medium such as punched cards or paper tape before it can be further processed. For this reason various methods of recording data directly in a computer-compatible state have been developed (see Burley, Andrew and Palmer, 1974). 
6.121 Standard forms for recording data

Forms suitable for recording field measurements in a species or provenance trial is illustrated in Appendix 8. A single form should be used for each experimental plot: although this may seem wasteful of paper, particularly if there are only a few trees assessed per plot, they do have the considerable advantage that forms can be arranged in field order (i.e. in the order in which plots are assessed) for recording and may be rearranged in whatever order is convenient for subsequent analysis or transcription. The need for transcription, and consequent transcription errors, is reduced to a minimum. There is the additional advantage that all measurements from one tree appear together in a single row of data, thus facilitating visual checking and comparison of information on each tree. When two or more characters are assessed together, height and diameter, say, it is convenient to record them together on the same form.

Each form is completely identified by the plot information appearing at the head of the page. Tree numbering should be consistent from assessment to assessment and the system should be specified in the experimental control plan as a simplified plot diagram indicating the sampling pattern and geographical orientation. Original tree numbers should be used throughout the life of the trial; dead or missing trees should be indicated by a dash $(-)$.

Although the forms illustrated in Appendix 8 are very general it will normally be convenient to prepare a specific edition for a particular trial, with the trial information pre-printed at the head of the form. As many copies of the forms as are required can then be printed on an office duplicator. This procedure saves the recorder having to fill in essential trial information.

All measurements should be recorded clearly and legibly so that any other officer can read them unambiguously. Ballpoint pen or dark pencil are recommended. The forms should be carried in the field in a clipboard or similar device providing a firm base and a secure hold. A waterproof cover for the forms may be useful. In very wet conditions waterproof forms and a $2 \mathrm{H}$ pencil prove satisfactory. Assessing and recording are best undertaken by a team of at least two people, one measuring and one recording. The assessor should call the measurement and the recorder call back the number he has written. This strategy reduces the possibility of errors in recording as well as being more convenient than a single officer attempting to both measure and record.

The forms illustrated in Appendix 8.3 will not be suitable for all types of assessment under all conditions, nor will they be compatible with all systems for analysis. A set of forms for other types of assessment is also included in Appendis 8. A11 forms may be modified according to local conditions and the advice of the person likely to be responsible for analysing data should be sought before any form is used.

\subsection{Other methods of recording data}

Manual copying or transcription of data is a tedious task, particularly if the work is properly checked. If it is not checked a moderate 
error rate is almost unavoidable. The following methods of recording data directly in a computer-readable form have been tried with varying levels of success.

(a) Pre-perforated cards. These are similar to an ordinary computer card except that only 40 of the 80 columns are used. The holes which correspond to numerical digits are already scored and may be punched by means of a metal stylus while the card is held in a device called a "Port-a-punch". This system has been quite widely used in forestry and elsewhere and is cheap. The main disadvantage of the system is that the cards can easily get wet, dirty and bent in a forest environment and once in that state cannot be read by the computer. Moreover, it is not so easy as with handwritten forms to check recording visually. The data on each card must be completely identified by plot and tree in case the cards get out of sequence, or a tree or plot is missed.

(b) Mark sense forms. These are paper forms which are precisely marked with boxes. A mark with a dark pencil in a box indicates a digit when the form is scanned by an optical reader. The forms are rather more flexible than pre-perforated cards as they can be specially prepared for a particular project, with printed information, making them easier to use and check. The system is more expensive than the "Port-a-punch" one as the optical reader must be purchased. The forms are also expensive as they have to be specially and precisely printed. Dirt on the form is a serious hazard and, since the forms are quite large (about A4 size although size restrictions are easing) it is difficult to keep them clean, dry and in one piece in the forest.

Currently interest centres on development of a portable machine for recording information on computer-readable magnetic tape. Such a device would be usable in all weathers and would incorporate a visual display of data recorded. However, development has been slow, partly because of the limited potential market.

Although not computer-compatible, the use of a portable tape recorder or dictation machine has much to recommend it, particularly where assessment and recording must be undertaken by one man. He can record his measurements and all necessary plot and tree identification and the equipment required is relatively cheap and unsophisticated. The disadvantages are that recordings cannot easily be checked tree by tree at the time of recording, and that manual transcription of the data is necessary.

Other aspects of data collection, storage and retrieval and analysis were discussed by Andrew and Burley (1973) and Burley, Andrew and Palmer (1974).

\subsection{Statistical analysis}

Data from a trial should be analysed as soon as possible. Not only does this prevent a large backlog of statistical work building up; the result of each analysis may suggest changes in the planning of treatment and assessment of the trial. 
Many different types and stages of analysis are possible (see Burley, 1973) but the methods of analysis used should be appropriate to the objectives of the experiment, to its design and to the traits being analysed. Most commonly in species and provenance trials the analysis of variance is used to measure the relative importance of variation between populations, systematic environmental variation and residual variation, and to estimate the precision with which population differences are calculated. Correlation and regression analysis are used to investigate the ways in which traits vary together. There are methods for analysing the effects of genotype by environment interactions. Multivariate techniques such as principal component analysis and cluster analysis are sometimes used to investigate the ways in which the populations differ in terms of all their measured traits.

Many of the methods mentioned above are complex and laborious to do by hand. If a computer is not readily available it is desirable to carry out some simple, preliminary analysis of the data, such as calculating and comparing plot and population mean values.

Whatever form of analysis is carried out it is essential to avoid errors which could lead to incorrect conclusions and, consequently, a wasted experiment or worse. All calculations should be carried out with sufficient accuracy to ensure that practical differences can be identified and to avoid rounding errors. Analyses carried out manually should always be checked and print-outs of computer analyses should not be accepted without ensuring that the program is reliable and the data are entered correctly.

\subsection{Preliminary analysis}

The simplest form of analysis, which should certainly be carried out as soon as possible, is the calculation of plot mean values. A plot mean is simply the arithmetic average, the sum of all measurements of a trait such as tree height, divided by the number of measurements:-

$$
\bar{x}=\sum_{i=1}^{n}\left(x_{i}\right) / n
$$

where $\bar{x}$ represents the mean of the $n$ individual heights $x_{i}$ and the symbol $\sum_{x=1}^{n}$ indicates the sum of $n$ items. Note that if, say, two trees are

missing from a 25 tree plot $\mathrm{n}$ is 23. P1ot means may be used to estimate population means and differences although if the experimental design is nonorthogona1 (incomplete blocks or lattice, say) the estimates may require adjustment for block differences. However, these preliminary estimates do show the way the crop is developing and should reveal any substantial differences between populations. 


\subsection{Transformations}

Most of the common methods of analysis require certain assumptions about the data. These are that residual variation is normally distributed and independent of plot means and that population, environmental and residual effects are additive. This last assumption implies that a plot mean in a RCB experiment can be represented as

$$
x_{i j}=m+b_{i}+p_{j}+e_{i j}
$$

where $x_{i j}$ is the plot mean, $m$ is the overall mean, $b_{i}$ is the effect of the $i^{\text {th }}$ block; $P_{i}$ the effect of the $j^{\text {th }}$ population and $e_{i j}$ is the residual effect of the ijth plot. In species and provenance trials most mature traits such as height and diameter satisfy the assumptions and can be analysed as recorded. Other measurements such as survival percentage and flowering count generally need to be transformed before analysis. The most frequently used transformations are the angular, square root and logarithmic.

(a) Angular transformation ("arcsine transformation"). This is applied to percentages lying between 0 and 100 which have a binomial distribution. This includes percentage of trees in a given class (e. $g$. diseased, forked, severely sinuous, etc.). Instead of the observed percentage $p$, the angle $\sin ^{-1}(\sqrt{\mathrm{p} / 100})$ is analysed; it has the effect of "stretching" the scale of measurement at the extremes (i.e. near $0 \%$ and $100 \%$ ) 80 as to equalize the variance over the whole range. Tables of the angular transformation are provided in Fisher and Yates (1963) and elsewhere.

(b) Square root transformation. This is used to equalize variance where it is proportional to the mean; this is a feature of counts with a Poisson-like distribution such as number of branches per node.

(c) Logarithmic transformation. The transformation of data to their logarithms is used where the variance is proportional to the square of the mean: this is often the case with assessment of flowering or of incidence of infestation or of certain types of damage. In such cases the assessment may be estimated visually using a series of classes which are effectively on a logarithmic scale. For example the following scale could be used: $1=1-5$ flowers per sample; $2=6-15 ; 3=16-$ $35 ; 4=36-75 ; 5=75-155$ and so on. In this case transformation is not required.

Apart from the angular transformation there is generally no need, in species and provenance trials data, to transform because of nonnormality of variation. This is because most traits other than percentages of whole plots are analysed on a plot mean basis. In this case the central limit thereom operates: this says that no matter how a trait is distributed from tree to tree, the distribution of averages of several trees is approximately normal. The approximation increases with sample size and is generally very close with ten or more trees. 
Transformations of various mathematical types are frequently used in regression analysis in order to linearize relationships.

\subsection{Analysis of variance}

The analysis of variance in individual traits is the most widely used method for interpreting the results of experiments. The analysis provides the following information:-

(i) estimates of the relative magnitude or importance of each identifiable source of variation;

(ii) estimated differences between populations and between environments;

(iii) indications of the accuracy of estimated differences by means of their standard errors and confidence limits;

(iv) tests of statistical significance of variances and differences.

Although the precise form of the analysis of variance varies according to the experimental design and the underlying mathematical model, the essential principle is to estimate the variance attributable to each source (blocks, populations, residual, etc.) of variation. Statistically significant variances are identified by means of the variance-ratio test (or F-test) and standard errors are derived from the variances in order to indicate the accuracy and statistical significance of estimated population (or environmental) differences. The analyses of variance for the designs most frequently used in species and provenance research are discussed below:- (a) fully randomized designs, (b) randomized complete block designs and (c) lattice designs. In all examples a random effects model is assumed. Tests of differences between means are discussed in Section 6.134.

(a) Fully randomized designs (single classification)

In this class of design there is only one identifiable systematic source of variation, that between populations. Any systematic environmental variation, which might otherwise be identified by means of blocking, is confounded with residual plot-to-plot variation. If there are p populations and $\mathrm{N}$ plots altogether (the replication of each population need not be constant) the analysis of variance is summarized as follows:-

\begin{tabular}{|l|c|c|c|c|}
\hline Source of variation & $\begin{array}{c}\text { Degrees of } \\
\text { freedom (df) }\end{array}$ & $\begin{array}{c}\text { Sum of } \\
\text { squares (SS) }\end{array}$ & $\begin{array}{c}\text { Mean square } \\
(\mathrm{MS})\end{array}$ & $\begin{array}{c}\text { Variance } \\
\text { ratio (F) }\end{array}$ \\
\hline Between populations (P) & $\mathrm{p}-1$ & $\mathrm{SS}_{\mathrm{P}}$ & $\mathrm{MS}_{\mathrm{P}}=\mathrm{SS}_{\mathrm{P}} /(\mathrm{p}-1)$ & $\mathrm{MS}_{\mathrm{P}} / \mathrm{MS}_{\mathrm{E}}$ \\
Residua1 (E) & $\mathrm{N}-\mathrm{p}$ & $\mathrm{SS}_{\mathrm{E}}$ & $\mathrm{MS}_{\mathrm{E}}=\mathrm{SS}_{\mathrm{E}} /(\mathrm{N}-\mathrm{p})$ & \\
\hline TOTAL & $\mathrm{N}-1$ & $\mathrm{SS}_{\mathrm{T}}$ & & \\
\hline
\end{tabular}


The calculation of degrees of freedom (i..e. the numbers of independent comparisons) for total variation and for differences between populations is straightforward; they are in each case one less than the number of values being compared. Residual degrees of freedom are found by subtraction of the population df from the total df.

Values in the sum of squares column are the sums of square deviation from the mean for each source of variation. Thus the total sum of squares is the sum of squared differences of the individual plot values from their overall mean and it may be calculated according to the formula:-

$$
\mathrm{SS}_{\mathrm{T}}=\sum_{i=1}^{\mathrm{N}}\left(\mathrm{x}_{\mathrm{i}}^{2}\right)-\frac{1}{\mathrm{~N}}\left(\sum_{i=1}^{\mathrm{N}} \mathrm{x}_{\mathrm{i}}\right)^{2}
$$

where the $x_{i}$ are individual plot values. This method is particularly suitable for use with a desk calculator, especially one having two accumulating registers: one for $\sum x$ and one for $\sum x^{2}$. However, since the result is calculated as the difference between large numbers it is essential to preserve accuracy by retaining as many figures as possible in the calculations. The quantity

$\frac{1}{N}\left(\sum_{i=1}^{N} x_{i}\right)^{2}$ is known as the correction factor $(C F)$ and is also used in the calculation of the sum of squares between populations:-

$S S_{P}=\sum_{j=1}^{p}\left(P_{j} 2 / r_{j}\right)-C F$.

In this formula the $P_{j}$ 's are the population totals and the $r_{j}$ 's represent the number of replicates of each population; thus each $P_{j}$ is the sum of $r_{j}$ plot values. The residual sum of squares is calculated as the difference between the total and population sums of squares.

Each mean square or variance is calculated as the ratio of the corresponding sum of squares and degrees of freedom. The statistical significance of the population mean square is tested by comparing the calculated variance ratio, $\mathrm{MS}_{\mathrm{P}} / \mathrm{MS}_{\mathrm{E}}$, with the critical values tabulated in $\mathrm{Fisher}$ and Yates (1963) and in many other statistical text books. The critical values used are those corresponding to the degrees of freedom available for populations and for residual and are usually tabulated for various levels of statistical significance, for example $p=0.05,0.01$ and 0.001 (respectively, $5 \%, 1 \%$ and $0.1 \%$ probabilities of wrongly inferring that real differences do exist between populations).

The precision and statistical significance of individual population mean values and differences may be examined by means of their standard errors:- 
The calculations for the analysis of variance are illustrated with an example and an adaptation of a Statform (Dawkins, 1968) in Appendix 9.1 .

\section{(b) Randomized complete blocks}

The analysis of variance for randomized complete block designs is only a little more complex than that for fully randomized experiments and is easily accomplished using a desk calculator. There are two identifiable systematic sources of variation: replications and populations; the accuracy and statistical significance of each is tested by comparison with the residual plot-to-plot variance. The analysis may be summarized as follows:-

\begin{tabular}{|l|c|c|c|c|}
\hline & $\begin{array}{c}\text { Degrees of } \\
\text { freedom (df) }\end{array}$ & $\begin{array}{c}\text { Sum of } \\
\text { squares } \\
(\mathrm{SS})\end{array}$ & Mean square (MS) & $\begin{array}{c}\text { Variance } \\
\text { ratio (F) }\end{array}$ \\
\hline Between replications (B) & $\mathrm{b}-1$ & $\mathrm{SS}_{\mathrm{B}}$ & $\mathrm{MS}_{\mathrm{B}}=\mathrm{SS}_{\mathrm{B}} /(\mathrm{b}-1)$ & $\mathrm{SS}_{\mathrm{B}} / \mathrm{MS}_{\mathrm{E}}$ \\
Between populations ( $\mathrm{P})$ & $\mathrm{p}-1$ & $\mathrm{MS}_{\mathrm{P}}=\mathrm{SS}_{\mathrm{P}} /(\mathrm{p}-1)$ & $\mathrm{SS}_{\mathrm{P}} / \mathrm{MS}_{\mathrm{E}}$ \\
\hline TOTAL ( T) & $(\mathrm{b}-1)(\mathrm{p}-1)$ & $\mathrm{MS}_{\mathrm{E}}=\mathrm{SS}_{\mathrm{E}} /((\mathrm{b}-1)(\mathrm{p}-1))$ & \\
\hline
\end{tabular}

where the experiment consists of $p$ populations fully replicated in b blocks, giving a total of bp plots. Degrees of freedom are calculated as shown; the residual df may also be calculated by differences. The total sum of square deviations is calculated by

$S S_{T}=\sum_{i=1}^{b} \sum_{j=1}^{p}\left(x_{i j}^{2}\right)-\frac{1}{b p}\left(\sum_{i=1}^{b} \sum_{j=1}^{p} x_{i j}\right)^{2}$

which is the difference between the sum of squares of the individual plot values and the correction factor, $C F=\frac{1}{b p}\left(\sum \Sigma x_{i j}\right)^{2}$.

The sums of squares for replications and for populations are

$S S_{B}=\frac{1}{p} \sum_{i=1}^{b}\left(B_{i}^{2}\right)-C F$

and $S S_{P}=\frac{1}{b} \sum_{j=1}^{p}\left(P_{j}^{2}\right)-C F$

where the $B_{i}$ and $P_{j}$ are replicate and population totals, respectively. The residual sum of squares is calculated by subtraction: $S_{E}=S_{T}-S_{B}-S_{P}$

Each mean square is calculated as the ratio of the corresponding sum of squares and degrees of freedom. The statistical significance of the 
variance between populations is tested by comparing its ratio to the residual mean square with the critical values tabulated in Fisher and Yates (1963) and elsewhere. The critical values used are those corresponding to the degrees of freedom for populations and for residual and they are usually tabulated for various levels of statistical significance, such as $\mathrm{p}=0.05,0.01$ and 0.001 (i... for $5 \%, 1 \%$ and $0.1 \%$ probabilities of inferring a real variation between populations where none exists). The variation between replicates (blocks) may be tested in a similar manner.

The standard errors of a population mean and difference are, respectively:-

$$
s_{\bar{x}}=\sqrt{ }\left(\mathrm{MS}_{E} / \mathrm{b}\right) ; s_{(\operatorname{diff})}=\sqrt{ }\left(2 \mathrm{MS}_{E} / \mathrm{b}\right) .
$$

Appendix 9.3 illustrates the calculations described here by means of an example and an adaptation of a Statform (Dawkins, 1968).

\section{(c) Lattice designs}

The analysis of data from lattice designs is rather more involved than that for fully randomized designs or randomized complete blocks and is best accomplished using an appropriate computer program. (A program for analysis of variance of fully and partially balanced square and rectangular lattices is available at CFI, Oxford). However, the computations can be undertaken, with care, on a desk calculator. The procedure for a partially balanced square lattice is described below. For a further discussion of the analysis of this and of other lattice designs see Cochran and Cox (1957).

The analysis may be summarized as follows:-

\begin{tabular}{|c|c|c|c|c|}
\hline Source of variation & $\begin{array}{l}\text { Degrees of } \\
\text { freedom }(\mathrm{df})\end{array}$ & $\begin{array}{l}\text { Sum of } \\
\text { s quares } \\
\quad \text { (SS) }\end{array}$ & $\begin{array}{l}\text { Mean square } \\
\text { (MS) }\end{array}$ & $\begin{array}{l}\text { Variance } \\
\text { ratio (F) }\end{array}$ \\
\hline $\begin{array}{l}\text { Between replications } \\
\quad(\mathrm{R})\end{array}$ & & $\mathrm{SS}_{\mathrm{R}}$ & $\mathrm{MS}_{\mathrm{R}}=\mathrm{SS}_{\mathrm{R}} / \mathrm{df_{ \textrm {R } }}$ & $\mathrm{MS}_{\mathrm{R}} / \mathrm{MS}_{\mathrm{B}}$ \\
\hline $\begin{array}{l}\text { Between populations, } \\
\text { unad } \mathrm{j} \text {. ( } P \text { ) }\end{array}$ & $k^{2}-1$ & $\mathrm{SS}_{\mathbf{P}}$ & $\mathrm{MS}_{\mathrm{P}}=\mathrm{SS}_{\mathrm{P}} / \mathrm{df_{ \textrm {P } }}$ & (see text) \\
\hline $\begin{array}{l}\text { Blocks in replications, } \\
\text { adj. (B) }\end{array}$ & $r(k-1)$ & $\mathrm{SS}_{\mathrm{B}}$ & $\mathrm{MS}_{B}=\mathrm{SS}_{\mathrm{B}} / \mathrm{df_{B }}$ & $\mathrm{MS}_{\mathrm{B}} / \mathrm{MS}_{\mathrm{E}}$ \\
\hline $\begin{array}{l}\text { Intra-block residual } \\
(E)\end{array}$ & $(k-1)(r k-k-1)$ & $\mathrm{SS}_{\mathrm{E}}$ & $M S_{E}=S S_{E} / d f_{E}$ & \\
\hline TOTAL (T) & $r k^{2}-1$ & $\mathrm{SS}_{\mathrm{T}}$ & & \\
\hline
\end{tabular}

where there are $r$ replications of a $k^{2}$ lattice (i.․․ $k^{2}$ populations arranged in $k$ incomplete blocks of $k$ plots each). The calculations are as follows:- 
1. Calculate the block totals $B$, the replicate totals $R$, the unadjusted population totals $\mathrm{P}$ and the grand total $\mathrm{G}$.

2. For each block compute $C=\sum_{i=1}^{k}(P(B) i)$ - $r B$; that is, the sum of the population totals for all the populations in that block, less $\mathrm{r}$ times the block total $B$. Find the replicate totals $R_{C}$ of the $C$ values. The $\mathrm{R}_{\mathrm{C}}$ totals should add to zero.

3. Calculate the correction factor $\mathrm{CF}=\mathrm{G}^{2} / \mathrm{rk}^{2}$ and the total sum of square $\mathrm{SS}_{\mathrm{T}}=\sum\left(\mathrm{y}^{2}\right)-\mathrm{CF}$; the replication sum of squares

$\mathrm{SS}_{\mathrm{R}}=\frac{1}{\mathrm{k}} \sum\left(\mathrm{R}^{2}\right)-\mathrm{CF}$ and the unadjusted population sum of squares $\mathrm{SS}_{\mathrm{P}}=\frac{1}{\mathrm{r}} \sum\left(\mathrm{P}^{2}\right)-\mathrm{CF}$.

4. The sum of squares for blocks, adjusted for population differences is

$\mathrm{SS}_{\mathrm{B}}=\frac{\sum\left(\mathrm{C}^{2}\right)}{\mathrm{kr}(\mathrm{r}-1)}-\frac{\sum \mathrm{R}_{\mathrm{c}}^{2}}{\mathrm{k}^{2} \mathrm{r}(\mathrm{r}-1)}$

5. The sum of squares for intra-block residual is found by subtraction.

6. Mean squares are calculated in the normal way, MS $=\mathrm{SS} / \mathrm{df}$ and the weighting factor, $\mu$, used to compute the adjusted population totals is given by

$\mu=\frac{M S_{B}-M S_{E}}{k(r-1) M S_{B}}$

If $\mu$ is negative (because $M S_{B}<M_{E}$ ) it is taken as zero and no adjustments are made to the population totals. The analys is may then be completed as if the design had been randomized complete blocks. The same action may be taken if $\mathrm{MS}_{B}$ is greater than $\mathrm{MS}_{E}$ but not statistically significantly so.

7. The block corrections are given by $\mu \mathrm{C}$. The adjusted population totals are found by adding to the unadjusted totals the net sum of the $\mu C$ values for each block in which a population appears. Unadjusted and adjusted populations means are given simply by dividing the corresponding totals by $r$.

8. The sum of square between adjusted population means is

$$
\mathrm{SS}_{\mathrm{P}}(\operatorname{adj})=\mathrm{SS}_{\mathrm{P}}-\mathrm{k}(\mathrm{r}-1) \mu\left\{\mathrm{SS}_{\mathrm{B}}(\operatorname{unadj})\left[\frac{r}{(r-1)(1+k \mu)}\right]-\mathrm{SS}_{B}\right\}
$$

in which the unadjusted sum of squares for blocks within replications, $\mathrm{SS}_{B}($ unadj $)=\Sigma\left(\mathrm{B}^{2}\right) / \mathrm{k}-\Sigma\left(\mathrm{R}^{2}\right) / \mathrm{k}^{2}$. The mean square for adjusted population means is tested against the intra-block residual mean square.

Alternatively an approximate test of population differences can be made by comparing the mean square for unadjusted population means ${ }^{M S}{ }_{P}$, with 
the "randomized complete block" residual mean square:-

$\mathrm{MS}_{\mathrm{E}}(\mathrm{RCB})=\frac{\mathrm{SS}_{\mathrm{B}}+\mathrm{SS}_{\mathrm{E}}}{(\mathrm{r}-1)\left(\mathrm{k}^{2}-1\right)}$

9. The standard error of a population difference varies according to whether the populations to be compared appear together in the same block or not. In practice there is not much difference and standard errors based on the effective residual variance:-

$\mathrm{MS}_{\mathrm{E}(\mathrm{L})}=\mathrm{MS}_{\mathrm{E}}\left(1+\frac{\mathrm{rk \mu}}{\mathrm{k}+1}\right)$

may be used. From this the standard errors of a population mean and difference are, respectively:-

$$
s_{(\text {mean })}=\left(\frac{{ }_{E S}(L)}{r}\right) ; s_{(\text {diff })}=\sqrt{\left(\frac{2 M S_{E(L)}}{r}\right)}
$$

10. The efficiency of the lattice design may be judged by comparing the effective residual variance, $M S_{E(L)}$ (given in 9 above), with the corresponding randomized complete block residual mean square, ${ }_{\mathrm{MS}}(\mathrm{RCB})$ given in 8 above). The efficiency, or relative accuracy, is Eff $=M_{E(R C B)} \div M S_{E(L)}$ and is usually expressed as a percentage.

(d) Other designs

The analyses of variance for other designs, such as latin squares, split-plot designs and various types of factorial design, are described in detail in several text books, for instance Snedecor and Cochran (1967), Cochran and Cox (1957) and Pearce (1965). Statforms, which provide an easy format to follow for computing the analysis of variance for these designs, similar to Appendices $9.1,9.3$ and 9.4 have been produced by Dawkins (1968).

\section{(e) Missing values in the analysis of variance}

Missing data may arise for a variety of reasons:- zero survival, incorrect laying down of a trial, incorrect treatment of one or more plots, errors in assessment or in recording data or loss of data. Most experimental designs incorporate a degree of orthogonality or of balance among the sources of variation and the existence of missing values upsets this. Consequently the analysis of data with missing values can be tedious. For the most commonly used designs there exist formulae for estimating missing values; these estimates are intended not so much to predict what the missing plot value would have been, but rather to complete the data and so give an analysis unbiassed by the existence of missing values.

For a single missing value in a randomized block design the formula is

$y=\frac{b B+p P-G}{(b-1)(p-1)}$ 
where there are $b$ blocks and $P$ populations, $B$ and $P$ are the totals for the block and population in which the missing value occurs and $G$ is the grand total. B, P and $G$ are calculated exclusive of the missing plot itself.

In a partially balanced lattice design, of the type discussed in 4.221 , the formula for a single missing value is

$y=\frac{(r-1) k^{2} p-r R+G-r k C+k C^{\prime}}{(r-1)(k-1)(r k-k-1)}$

in which there are $r$ replicates and $k^{2}$ populations, $R$ and $P$ are the totals for the replicate and population in which the missing value occurs and $G$ is the grand total. $C$ is the $C$ value (see Section $6.133 \mathrm{c}$ ) for the block with the missing observation and $\mathrm{C}^{-}$is the total of all the $\mathrm{C}$ values for all blocks which contain the population with the missing value (Cochran and Cox, 1957). All totals are calculated exclusive of the missing plot.

Missing value formulae for other designs are given by Cochran and Cox (1957), and by Snedecor and Cochran (1967). Note that since there is no orthogonality or balance in a fully randomized design missing values are not estimated but simply omitted.

If there are two or more missing values in a set of data they must be estimated iteratively as follows:-

(i) Insert reasonable guessed values for all but one of the missing plots.

(ii) Use the appropriate formula to estimate the remaining value.

(iii) Repeat the process for each missing plot in turn, substituting estimated for guessed values.

(iv) Repeat the whole sequence (steps (i)-(iii)) until successive estimates of each missing value barely differ.

Where there are many missing values the iterative process is very lengthy and therefore impractical except on an electronic computer. If a computer and program are not available and there are many missing values it may be necessary to analyse the experiment as a fully randomized design.

The total and residual degrees of freedom in the analysis of variance should each be reduced by one for every missing value.

\subsection{Comparison of means, tests of differences}

The first and simplest way to compare population mean values is to rank them in order of magnitude. In this way patterns of variation and grouping of populations may readily present themselves. If an F-test reveals statistically significant variation among the populations, however, it is desirable to identify the exact source(s) of that variation. It is also frequently necessary to test the differences between 
individual populations or groups of populations. Several methods exist for these purposes and are described briefly below.

\section{(a) Least significant difference ( $t$ test)}

The difference between two populations may be inferred statistically significant if it exceeds the least significant difference LSD $=t x s_{(\text {diff) }}$, i.e. the standard error of the population difference multiplied by the tabulated value of Student's $t$ for the appropriate residual degrees of freedom and for the level of significance (generally $p=5 \%$ or $1 \%$ ) at which the test is made. Tables of $t$ are given in most text books and sets of statistical tables. The test may also be used for comparing groups of populations: a provenance trial may for example include seed sources in two or more identifiable "regions". In this case the stanuard error of the difference between the means of two groups is

$s_{(\operatorname{diff})}=\sqrt{ }\left(\frac{{ }_{\mathrm{MS}}}{\mathrm{r}}\left(\frac{1}{\mathrm{n}_{1}}+\frac{1}{\mathrm{n}_{2}}\right)\right)$

in which $n_{1}$ and $n_{2}$ are the numbers of populations in each group.

The test, though simple, is only valid for population comparisons selected before inspection of the data (Cochran and Cox, 1957, pp. 73-4) and should not be used in a multiple comparison of ranked population means. Special tests have been devised for this situation and the two most commonly used are described below.

Individual comparisons that were not anticipated before the data were collected can be tested using a method due to Scheffe. An example of the computations involved is given in Appendix 9.2.

(b) Studentized range test (Tukey) and Duncan's multiple range test

Tests of differences between populations which have been ranked in order of magnitude of their mean values, or tests of differences which are selected because they are large or extreme, should be made using a method such as the Studentized range test or Duncan's multiple range test which have been designed for the purpose. With either test the procedure is as follows : -

1. Rank the population means in order of magnitude.

2. To test the difference among a sequence of $k$ mean values compute the critical difference:$c={ }_{v}^{k} Q_{p} \times s_{(m e a n)}$

in which ${ }_{v}^{k} Q_{p}$ is the tabulated value of the studentized range or of Duncan's test statistic for a range of $k$ means, $v$ residual degrees of freedom and significance level p. Note that the standard error of a population mean is used, not that of a difference.

3. The test is usually applied sequentially to identify groups of mean values which do not differ significantly. Such groups may be identified 
by a bar spanning them, $\underline{e} \cdot \underline{g}$.

\begin{tabular}{lllll}
20.3 & 19.6 & 16.4 & 15.8 & 15.1 \\
\hline
\end{tabular}

It should be noted that the two tests give slightly different critical differences and can therefore give different inferences. This is because the tests vary a little in the protection they give against wrongly inferring significant differences, for a given level of significance. In practice there is little to choose between the tests.

Tables of the Studentized range are given in Snedecor and Cochran (1967) in Pearson and Hartley (1966) and in Rohlf and Sokal (1969). Tables of Duncan's multiple range test criterion were published by Duncan (1955).

\subsection{Components of variance}

Each mean square in the analysis of variance is made up of one or more of the components due to the different identifiable sources of variation. Thus for example, the mean squares in a randomized complete blocks analysis are theoretically composed as follows:-

\begin{tabular}{|l|c|c|}
\hline Source of variation & $\mathrm{df}$ & $\begin{array}{c}\text { Expected Mean Square } \\
\mathrm{E}(\mathrm{MS})\end{array}$ \\
\hline Replicates & $\mathrm{b}-1$ & $\mathbf{s}_{\mathrm{E}}^{2}+\mathrm{p} \mathrm{s}_{\mathrm{B}}^{2}$ \\
Populations & $\mathrm{p}-1$ & $\mathrm{~s}_{\mathrm{E}}^{2} \mathrm{~b} \mathrm{~s}_{\mathrm{P}}^{2}$ \\
Residual & $(\mathrm{b}-1)(\mathrm{p}-1)$ & 2 \\
\hline
\end{tabular}
$s_{\mathrm{p}}^{2}$ is the component for population differences and $\mathrm{s}_{\mathrm{E}}^{2}$ is the residual variance component due to component for variation between replicates. Estimates of $s_{\mathrm{E}}, s_{\mathrm{B}}^{2}$ and $s_{\mathrm{P}}^{2}$ can be computed by comparing expected mean squares with calculated values. Thus, in the randomized block analysis, estimates are:-

$s_{B}^{2}=\left(M S_{B}-M S_{E}\right) / p ; s_{P}^{2}=\left(M S_{P}-M_{E}\right) / b$ and $s_{E}^{2}=M S_{E}$

It sometimes happens that negative estimates of $s_{\mathrm{B}}^{2}$ or $s_{\mathrm{P}}^{2}$ are obtained becuase, say, $\mathrm{MS}_{\mathrm{P}}$ is less than $\mathrm{MS}_{\mathrm{E}}$. since the components are variances they cannot, in theory, be negative and various procedures exist for dealing with such a situation. The simplest, though not entirely accurate, is to treat negative components as zero.

Variance components for a fully randomized design and for a square lattice are given below:-

(a) Fully randomized design

\begin{tabular}{|l|c|c|}
\hline Source of variation & $\mathrm{df}$ & $\mathrm{E}(\mathrm{MS})$ \\
\hline Populations & $\mathrm{P}-1$ & $\mathrm{~s}_{\mathrm{E}}^{2}+\frac{\mathrm{N}}{\mathrm{P}} \mathrm{s}_{\mathrm{P}}^{2}$ \\
Residual & $\mathrm{N}-\mathrm{P}$ & $\mathrm{s}_{\mathrm{E}}^{2}$ \\
\hline
\end{tabular}




\begin{tabular}{|l|c|c|}
\hline Source of variation & $\mathrm{df}$ & \multicolumn{1}{|c|}{$\mathrm{E}(\mathrm{MS})$} \\
\hline Replications & $\mathrm{r}-1$ & $\mathrm{~s}_{\mathrm{E}}^{2}+\mathrm{k} \mathrm{s}_{\mathrm{B}}^{2}+\mathrm{k}^{2} \mathrm{~s}_{\mathrm{R}}^{2}$ \\
Populations (adjusted) & $\mathrm{k}^{2}-1$ & $\mathrm{~s}_{\mathrm{E}}^{2}+\mathrm{r} \mathrm{s}_{\mathrm{P}}^{2}$ \\
Blocks in reps (adjusted) & $\mathrm{r}(\mathrm{k}-1)$ & $\mathrm{s}_{\mathrm{E}}^{2}+\mathrm{k} \mathrm{s}_{\mathrm{B}}^{2}$ \\
Intra-block residual & $(\mathrm{r}-1)(\mathrm{rk}-\mathrm{k}-1)$ & $\mathrm{s}_{\mathrm{E}}^{2}$ \\
\hline
\end{tabular}

Note that the adjusted population mean square is used for estimating $s_{\mathrm{P}}^{2}$. An alternative estimate could be derived by computing the appropriate randomized complete block analysis, $\underline{i}$.e. by comparing the unadjusted population mean square with the randomized block residual mean square.

The principal use for variance components in tree improvement studies is in estimating genetic parameters within populations and for predicting genetic gain through selection. Heritability, or experimental repeatability, may be calculated as $\mathrm{h}^{2}=$ (genetic variance) $\div$ (genetic + environmental variance). For the designs considered in detail here, estimates of $\mathrm{h}^{2}$ are as follows:-

(i) Fully randomized: $h^{2}=s_{P}^{2} /\left(s_{P}^{2}+\frac{p}{N} s_{E}^{2}\right)$

(ii) Randomized complete blocks: $h^{2}=s_{P}^{2} /\left(s_{P}^{2}+s_{E}^{2} / b\right)$

(iii) Square lattice: $h^{2}=s_{P}^{2} /\left(s_{P}^{2}+s_{E}^{2} / r\right)$.

Typically, estimated values of $h^{2}$ vary considerably for different measured traits; desirable values are about 0.5 or greater (the maximum possible value is 1.0 , of course) $/ \underline{1}$.

The calculation and use of variance components in sampling strategies are discussed in Section 6.224.

\subsection{Analysis of covariance}

Covariance analysis is an extension of the analysis of variance in which observed plot values are adjusted according to their correlation with initial values or with inherent site variability. Thus growth in some plots may be good partly because of high soil fertility; in this case assessment of some aspect of fertility may be used as a covariate to adjust the analysis of variance. (The effects of site variability should, of course, be controlled as far as possible by good experimental design; covariance analysis provides a means of reducing residual variation which cannot be controlled by design alone.) The theory behind the analysis of covariance is, strictly, only applicable if the covariate is independent of population differences, as in the example just described. The method may also be used to adjust, say, fifth year growth data according to variation in initial field height, in order to investigate, specifically,

/1 Care must be used in the treatment and application of heritability values and, normally, they are not computed for provenance studies (but see Nanson, 1970). 
growth in the field. In this sort of analysis the standard significance tests should not be made as they may be seriously in error (Cochran and Cox, 1957); moreover the results of the analysis must be interpreted with extreme care. If adjustment makes little difference to ultimate population differences it indicates that initial growth differences between populations do not have much practical importance. If initial growth is, on the other hand, closely related to ultimate performance the adjustment by the analysis of covariance may obscure important population differences; a provenance may ultimately perform better than its rivals precisely because it grows well initially.

Computations for the analysis of covariance for simple designs are described by Snedecor and Cochran (1967) and by Cochran and Cox (1957). A Statform for the calculation of a randomized block analysis of covariance is given by Dawkins (1975). For square lattice designs the analysis is described by Cox, Eckhardt and Cochran (1940).

\subsection{Correlation and regression}

The simple correlation coefficient $r$ measures the extent to which two measured traits $X$ and $Y$ vary linearly together. That is, if $n$ values of $X$ and $Y$ are plotted on a graph, or scatter diagram, the value of $r$ indicates the closeness of the scatter to a straight line. The correlation coefficient is estimated as

$$
r=\frac{S P_{x y}}{\sqrt{S S_{x} \times / S S_{y}}}
$$

where the sums of squares of deviations of $X$ and $Y$ are

$$
S S_{x}=\sum_{i=1}^{n}\left(x^{2}\right)-\frac{1}{n}\left(\sum_{i=1}^{n} X\right)^{2} ; S S_{y}=\sum_{i=1}^{n}(Y)^{2}-\frac{1}{n}\left(\sum_{i=1}^{n} Y\right)^{2}
$$

and the sum of deviation cross products is

$$
S P_{x y}=\sum_{i=1}^{n}(X Y)-\frac{1}{n} \sum_{i=1}^{n} X \sum_{i=1}^{n} Y
$$

Estimates of $r$ may vary from -1 to +1 ; the extreme values indicate respectively perfect negative or positive correlation, $\underline{i}$.e. all points on a straight line sloping downwards ( $Y$ decreasing as $X$ increases) or upwards ( $\mathrm{X}$ and $\mathrm{Y}$ increasing together). Estimates of $\mathrm{r}=0$ imply a random scatter or no relationship at all between values of the two traits. In practice estimates usually fall between 0.1 and \pm 1 , indicating some relationship and some random variation; the statistical significance of a correlation coefficient can be determined from a table such as that given by Fisher and Yates (1963).

The form of a relationship between two traits may be further investigated by means of simple regression analysis. The straight line regression line $\mathrm{Y}=\mathrm{a}+\mathrm{bX}$ can be estimated and used to predict and explain variation in $Y$ according to variation in $X$. Estimates of the regression coefficients are 


$$
b=\frac{S_{x y}}{S S_{x}} \text { and } a=\bar{Y}-b \bar{X}
$$

where $\mathrm{SP}_{\mathrm{xy}}$ and $\mathrm{SS}_{\mathrm{x}}$ are calculated as described above and the estimate of the constant term $a$ is derived from the mean values, $\bar{Y}$ and $\bar{X}$, of the variables and the estimated value of $b$.

Normally it is the slope coefficient b which is of greatest interest in regression analysis; estimates may take any value depending on the scales of measurement of $X$ and $Y$. Positive values of $b$ indicate $X$ and $Y$ increasing together, negative values imply an inverse relationship while a zero, or near-zero, estimate means there is no linear relationship between the variables. The statistical significance of $b$ can be tested by $a$ ' $t$ ' test, comparing the ratio $b / s$ with tabulated values of student's $t$. The standard error of $b, b_{b}$ is estimated by

$$
s_{b}=\frac{s s_{y}-b^{2} s s_{x}}{(n-2) s s_{x}}
$$

The "goodness of fit" of the regression line is given by the square of the correlation coefficient. $r^{2}$ is known as the coefficient of determination and indicates the proportion of variation in $\mathrm{Y}$ which is explained by a linear function of the explanatoryvariable $X$. Desirable values of $r^{2}$ vary according to circumstances but typically a value of under about 0.6 is of little use for explanation or prediction of $Y$. The $r^{2}$ statistic is frequently expressed as a percentage.

A poor regression may be a consequence of trying to fit a straight line to a nonlinear relationship. The probable form of a relationship should be investigated initially by means of a simple scatter diagram; strong but nonlinear relationships may then be fitted by suitably transforming the $Y$ or $X$ values, or both. It is impossible to give hard and fast rules for transforming regression data but frequently the squares, square roots or logarithms of variables are used to linearize a curvilinear relationship. The regression of $Y$ and $\log X$ may be estimated if, for example, variation of $\mathrm{Y}$ with $\mathrm{X}$ was less pronounced at higher values of $\mathrm{X}$ than at lower values. Alternatively a quadratic regression may be fitted:-

$$
\mathrm{Y}=\mathrm{a}+\mathrm{b}_{1} \mathrm{X}+\mathrm{b}_{2} \mathrm{x}^{2}
$$

in which the effect of the linear component $b_{1}$ is modified by the quadratic component, $b_{2}$. In the example just quoted estimates of $b_{1}$ and $b_{2}$ would be opposite in sign.

Quadratic regression is a special case of multiple regression:-

$$
\mathrm{Y}=\mathrm{a}+\mathrm{b}_{1} \mathrm{X}_{1}+\mathrm{b}_{2} \mathrm{X}_{2}+\ldots \ldots \ldots \ldots \ldots \ldots \mathrm{b}_{\mathrm{m}} \mathrm{X}_{\mathrm{m}}
$$

where variation in $\mathrm{Y}$ is explained by the combined effects of $\mathrm{m}$ separate $X$ variables. In general estimation of the regression coefficients and their standard errors is a very lengthy operation and is best done on a computer. The relatively simple calculations for two $X$ variables are described in Snedecor and Cochran (1967) and elsewhere. Interpretation 
of multiple regression equations is a straightforward extension of that for the simple regression case; the effect of each variable may be examined by comparing its b coefficient and the corresponding standard error; the overall goodness of $f$ it is measured by the coefficient of multiple determination $R$ (conventionally, a capital ' $R$ ' in the multiple regression situation). Strictly the $X$ variables should be mutually independent though this can rarely be achieved in practice. If the correlation between two or more $X$ variables exceeds that between the X's and $Y$ the regression coefficients $b$ will be poorly estimated with large standard errors. The set of explanatory variables should never include two or more $X^{\prime} s$ which are exactly related to each other (e.g. rain, sumer rain and total rain) as the calculations will either fail completely or give nonsensical results.

\subsection{Series of experiments: genotype-environment interactions}

Species and provenance trials are often planted on several sites. Within a single country the range of experimental sites may include very widely different environmental conditions and it is likely that the relative performance of the populations tested will vary from site to site. For example, one provenance may grow well on a wet site and poorly on a dry site, compared with other provenances. Another provenance may adapt well to the dry site while a third may grow relatively well on both sites. Genotype-environment interaction is the extent to which a genotypic effect, measured by the average response over all sites of a population, and an environmental effect are not additive. Environmental effects may be measured by the average response of all populations at each site, or by a control population, or they may be expressed as functions of site conditions such as rainfall, temperature and soil quality.

The magnitude of a genotype-environment interaction may be estimated by means of an analysis of variance. If $p$ populations are represented at $s$ sites in randomized complete block experiments, each with b blocks, the analysis may be summarized as follows:-

\begin{tabular}{|l|l|l|l|l|}
\hline Source of variation & \multicolumn{1}{|c|}{$\mathrm{df}$} & $\mathrm{SS}$ & $\mathrm{MS}$ & $\begin{array}{c}\text { Variance } \\
\text { ratio }\end{array}$ \\
\hline Between sites & $\mathrm{s}-1$ & $\mathrm{sS}$ & $\mathrm{MS}_{\mathrm{S}}$ & $\mathrm{MS}_{\mathrm{S}} / \mathrm{MS}_{\mathrm{B}}$ \\
Blocks in sites & $\mathrm{s}(\mathrm{b}-1)$ & $\mathrm{SS}_{\mathrm{B}}$ & $\mathrm{MS}_{\mathrm{B}}$ & $\mathrm{MS}_{\mathrm{B}} / \mathrm{MS}_{\mathrm{R}}$ \\
Between populations & $\mathrm{p}-1$ & $\mathrm{SS}_{\mathrm{P}}$ & $\mathrm{MS}_{\mathrm{P}}$ & $\mathrm{MS}_{\mathrm{P}} / \mathrm{MS}_{\mathrm{R}}$ \\
Site x population interaction & $(\mathrm{s}-1)(\mathrm{p}-1)$ & $\mathrm{SS}_{\mathrm{SP}}$ & $\mathrm{MS}_{\mathrm{SP}}$ & $\mathrm{MS}_{\mathrm{SP}} / \mathrm{MS}_{\mathrm{R}}$ \\
Residua1 & $\mathrm{s}(\mathrm{b}-1)(\mathrm{p}-1)$ & $\mathrm{SS}_{\mathrm{R}}$ & $\mathrm{MS}_{\mathrm{R}}$ & \\
\hline TOTAL & $\mathrm{sbp}-1$ & $\mathrm{SS}_{\mathrm{T}}$ & & \\
\hline
\end{tabular}

Calculation of the sums of squares of deviations is straightforward. If raw data ( $p$ lot values) are $y$, then block totals $B_{i j}=\sum_{k=1}^{p} Y_{i j k}$ 
and site totals $s_{i}=\sum_{j=1}^{b} \sum_{k=1}^{p} Y_{i j k}$. Let population totals at each site $G_{i k}=\sum_{i=1}^{b} Y_{i j k}$, so overall population totals $P_{k}=\sum_{i=1}^{s}\left(G_{i k}\right)$.

The sums of squares follow:-

$$
\begin{aligned}
& \text { Correction factor, } \mathrm{CF}=\left(\sum \mathrm{y}\right)^{2} / \mathrm{sbp} \\
& \text { Total SS, } \mathrm{SS}_{\mathrm{T}}=\sum\left(\mathrm{y}^{2}\right)-\mathrm{CF} \\
& \text { Site SS, } \mathrm{SS}_{\mathrm{S}}=\frac{1}{\mathrm{bp}} \sum\left(\mathrm{S}^{2}\right)-\mathrm{CF} \\
& \text { Block SS, } \mathrm{SS}_{\mathrm{B}}=\frac{1}{\mathrm{P}} \sum\left(\mathrm{B}^{2}\right)-\frac{1}{\mathrm{bp}} \Sigma\left(\mathrm{s}^{2}\right) \\
& \text { Population SS, } \mathrm{SS}_{\mathrm{P}}=\frac{1}{\mathrm{~s}} \Sigma\left(\mathrm{P}^{2}\right)-\mathrm{CF} \\
& \text { Site x Population SS, SS } \mathrm{SP}=\frac{1}{\mathrm{~b}} \Sigma\left(\mathrm{G}^{2}\right)-\mathrm{CF}-\mathrm{SS}_{\mathrm{S}}-\mathrm{SS}_{\mathrm{P}}
\end{aligned}
$$

The residual sum of squares is found by subtraction and the mean squares are estimated by dividing each sum of squares by the corresponding degrees of freedom. Variance ratio estimation is indicated in the table.

Analysis of variance of series of experiments is more complex for designs such as lattices, or where experimental design or size varies from site to site. A simplified analysis using population means (adjusted, if appropriate) at each site is summarized below:-

\begin{tabular}{|l|c|l|l|}
\hline Source of variation & $\mathrm{df}$ & $\mathrm{SS}$ & $\mathrm{MS}$ \\
\hline Between sites & $\mathrm{s}-1$ & $\mathrm{SS}_{\mathrm{S}}$ & ${ }^{\mathrm{MS}} \mathrm{S}$ \\
Between populations & $\mathrm{p}-1$ & $\mathrm{SS}_{\mathrm{P}}$ & ${ }_{\mathrm{MS}}{ }_{\mathrm{P}}$ \\
Residual (site x pop. interaction) & $(\mathrm{s}-1)(\mathrm{p}-1)$ & $\mathrm{SS}_{\mathrm{SP}}$ & ${ }^{\mathrm{MS}} \mathrm{SP}$ \\
\hline TOTAL & $\mathrm{sp}-1$ & $\mathrm{SS}_{\mathrm{T}}$ & \\
\hline
\end{tabular}

The total sum of squares is estimated by $\mathrm{SS}_{\mathrm{T}}=\sum\left(\mathrm{y}^{2}\right)-\mathrm{CF}$ where the correction factor $\mathrm{CF}=\frac{1}{\mathrm{~S}}\left(\sum \mathrm{y}\right)^{2}$. Sums of squares for sites and for populations are, respectively,

$$
\mathrm{SS}_{\mathrm{S}}=\frac{1}{\mathrm{P}} \Sigma\left(\mathrm{S}^{2}\right) \text { and } \mathrm{SS}_{\mathrm{P}}=\frac{1}{8} \Sigma\left(\mathrm{P}^{2}\right) \text { where } \mathrm{S} \text { and } \mathrm{P} \text { are site and popu- }
$$
lation totals. The residual sum of squares, which provides an estimate of site-population interaction, is found by subtraction. An approximate test of overall site and population effects can be made by calculating the variance ratios $\mathrm{MS}_{\mathrm{S}} / \mathrm{MS}_{\mathrm{SP}}$ and $\mathrm{MS}_{\mathrm{P}} / \mathrm{MS}_{\mathrm{SP}}$; the validity of the test depends on the magnitude of non-estimated variation between blocks and between plots.

It is sometimes useful to relate the performance of each population to variation in the environment between sites. This can be done by simple regression analysis of $y=a+b x$ where $y=$ mean performance of the population at each site, $x=$ effect of environment at each site and $a$ and $b$ are the regression constant term and slope coefficients. If $x$ is measured by the overall site mean performance it is a simple matter to describe a 
population in terms of its slope coefficient. A slope b=1 implies typical or average improvement in performance with improved environment; such a population has average stability. An estimate of b significantly greater than unity implies a population in which the response to environmental variation is greater than average; such a population may only be suited to good sites. Slope coefficients less than one are associated with populations which are relatively unaffected by site environment (í.e. stable populations). Populations which are to be planted on a wide variety of site types should have a low slope coefficient combined with a high overall mean response.

Regression of population performance on environmental variation may be used to explain the site-population interaction term in the analysis of variance of data from series of experiments. The technique, known as joint regression analysis, has been described by various writers, including Freeman and Perkins (1971), Mergen, Burley and Furnival (1974).

\subsection{Multivariate analysis}

The combined variation in several observed traits may be investigated by principal component analysis (PCA). This is an exploratory method which summarizes the total variation by means of a set of mutually independent linear transformations of the $m$ original, correlated variables, $y_{i}$. The coefficients $a_{1 i}$ of the first principal component

$$
a_{11} y_{1}+a_{12} y_{2}+\ldots \ldots \ldots \ldots \ldots \ldots \ldots a_{1 m} y_{m}
$$

are chosen so as to maximize the variance of the component, $\underline{i} \cdot \underline{\text { e. }}$ to account for as much as possible of the total variation. The second principal component is fitted so as to account for as much as possible of the remaining variation and to be independent of (uncorrelated with) the first component. Subsequent components are estimated in turn to be independent of all previous components and to explain as much as possible of variation unexplained by those other components. Mathematically the principal components are given by the latent vectors of the correlation matrix of the original variables (or of their covariance matrix - see below). Variances of the components are given by the corresponding latent roots and are often expressed as a percentage of total variation. In general $\mathrm{m}$ components are required to account for all the variation in $\mathrm{m}$ variables but it is generally found that a relatively small number of components will account for most $(75 \%-90 \%)$ of the original variation. It may be much easier to interpret variation in, say, two to four principal components than that in ten or a dozen correlated traits. The number of observations (populations) $n$ should exceed the number of variables $m$. If $n \leqslant m$ only $n-1$ components can be estimated, and interpretation of these may be misleading.

In addition to summarizing variation in observed traits the components may provide evidence of the underlying structure of variation. This is done by consideration of the relative magnitudes of the component coefficients $a_{k i}$. These are commonly presented in one of the three ways:as correlations (loadings) between the $k^{\text {th }}$ component and the $i^{\text {th }}$ variable; as coefficients of standardized components (i.e. scaled to give each component unit variance); or scaled so that the largest coefficient in each 
component has a value of +1 . In each case a large coefficient implies the component is relatively closely associated with variation in the corresponding variable; typically each component represents (is composed of) combined variation in one or more of the original traits. If, for example, two of those traits were tree height (H) and diameter (D) a component with large positive loadings on $\mathrm{H}$ and $\mathrm{D}$ might be interpreted as representing variation in overall vigour. Another component, with large positive loading on $H$ and large negative loading on $D$ could be interpreted as variation in stem form.

Since the largest principal components are chosen to maximize variation among the observations (populations) it is often useful to plot the individual population values of the components as a means of identifying overall differences and similarities between the populations. Twodimensional graphs of the first and second, first and third, and second and third components are usually sufficient for this purpose. The component values are estimated by inserting the relevant population values $y_{i}$ of the $m$ variables into the component transformation

$$
a_{k 1} y_{1}+a_{k 2} y_{2} \ldots \ldots \ldots \ldots \ldots+a_{k m} y_{m} \cdot \text { In practice standardized }
$$

values of the original traits should be used as principal component analysis is usually based on standardized data, i.e. the original traits are scaled to zero mean and unit variance. Components derived from untransformed data will differ according to the relative scales of measurement of the original traits. Components derived from standardized or from unstandardized data are sometimes described as being based on correlations or on covariances, respectively.

Principal component analysis, in common with other multivariate techniques, cannot easily be accomplished on a desk calculator, except for a trivial number of variables. An electronic computer is essential and for this reason no guide to computation is given here. An elementary guide to the calculations is given by Pearce (1965); more details of this and other multivariate techniques are described by Seal (1964). 
6.2 Sample design for research in natural stands or plantations

H.L. Wright and P.G. Adlard

\subsection{Introduction}

The need to sample is obvious since one cannot always measure the entire population; good measurements on $10 \%$ of the population may well be more reliable than bad measurements on $100 \%$ of the population. The basic concept in sampling is the population which may be defined as any set of individuals (or objects) having some common observable characteristic. The term population may refer either to the individuals measured or to the measurements themselves. For example one may have a 10 ha forest in which there is a population of individual trees; the diameters of all the trees constitute another population. Alternatively the population could consist of 10 plots of 1 ha or 100 plots of 0.1 ha, etc.

A population is characterized by certain constants, e.g. the mean and the variance of the mean, which are known as parameters. The object of sampling is to estimate these parameters.

The principal stages involved in the planning and execution of a sample survey are:-

(a) Objectives. A clear statement is required since it is easy to lose sight of the objectives when planning a large survey.

(b) Definition of the population to be sampled.

(c) Data to be collected. Ensure that all the data are relevant and avoid the tendency to collect unnecessary data.

(d) Methods of measurement. Prescribe the measurement procedures.

(e) Degree of precision required. This affects the size of the sample.

(f) Selection of sample. This involves the choice of sampling unit, the division of the population into sampling units, the choice of sample size and the design.

(g) Organization of field work.

(h) Analys is and summary of the data.

(i) Results and conclusions.

\subsection{Sample design}

A useful reference is Elementary Forest Sampling by Freese, 1962) whilst a more theoretical reference is Sampling Techniques (Cochran, 1965).

The design of a sample is the way in which the sampling units are chosen from the population. Sampling designs may be broadly classified as shown below:- 


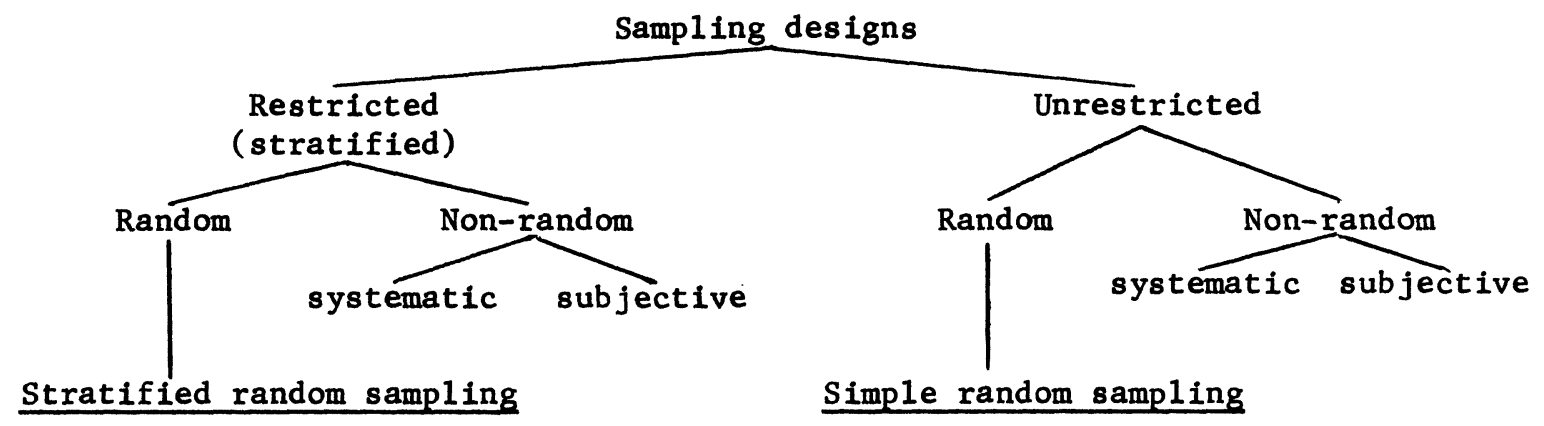

\subsection{Simple random sampling}

This method is the basis of most sampling designs. Given a population divided into $\mathrm{N}$ sampling units (individual trees, 0.1 ha plots, $1 \mathrm{~m}^{2}$ quadrats, etc.) simple random sampling is a method of selecting $n$ units out of the $\mathrm{N}$ in such a way that every possible combination of $n$ units has an equal chance of being selected. The selection of any given unit should be completely independent of the selection of all the other units.

In practice a simple random sample is drawn unit by unit. Every unit in the population is numbered from 1 to $\mathrm{N}$ and a series of random numbers is then selected from a table of random numbers. The units bearing these numbers constitute the sample.

The units may be selected with replacement or without replacement; in the latter case, which is most common, a unit is allowed to appear in the sample only once.

The calculations are simple and straightforward:-

Mean: $\quad \bar{x}=\frac{\sum x_{i}}{n}$

where the $x_{i}$ are the $n$ individual measurements.

Variance: a measure of the dispersion of the individual unit values about the mean value.

$$
s_{x}^{2}=\frac{\left(x_{i}-\bar{x}\right)^{2}}{n-1}=\frac{\sum x_{i}^{2}-\frac{\sum\left(x_{i}\right)^{2}}{n}}{n-1}
$$

Standard deviation: the square root of the variance.

$$
s_{x}=\sqrt{s_{x}^{2}}
$$

Variance of the mean:

$$
s \frac{2}{x}=\frac{s_{x}^{2}}{n}
$$

Standard error of the mean:

$$
s_{\bar{x}}=\frac{s x}{\sqrt{N}}
$$


Coefficient of variation: the ratio of the standard deviation to the mean $\mathrm{CV}=\frac{\mathrm{s}}{\overline{\mathrm{x}}}$, usually expressed as a percentage. It puts the measure of variability on a relative basis since the coefficient is independent of the scale or size of the mean value.

Confidence 1imits: Some idea of the precision of the estimated mean is usually required. This is provided by confidence limits giving the range in which the true value is expected to fall with a given level of probability.

$\mathrm{x} \pm \mathrm{ts}-$

where $t$ is the value of Student's $t$ at the chosen probability level and for the appropriate degrees of freedom $(n-1)$.

Sampling error: The efficiency of a sample is often measured by the sampling error percent

$$
\frac{100 \cdot \mathrm{ts}_{\overline{\mathrm{x}}}}{\overline{\mathrm{x}}}
$$

All the above formulae assume that the population is normally distributed. In the case of sampling without replacement, the variance of the mean is adjusted by a factor known as the finite population factor

$$
(1-n / N)
$$

This may be ignored when the sampling fraction $(n / N)$ is less than 0.05 or $5 \%$.

Sample size: Provided that an estimate of the population standard deviation or coefficient of variation is available, the sample size to achieve a given precision may be calculated using:

$$
n=\frac{t^{2} s^{2}}{E^{2}} \quad \text { or } n=\frac{t^{2} \mathrm{CV}^{2}}{P E^{2}}
$$

where $t$ is Student's $t$, with $(n-1)$ degrees of freedom, $s$ is the standard deviation, $\mathrm{E}$ is the desired error, $\mathrm{CV}$ is the coefficient of variation percent and $\mathrm{PE}$ is the desired percentage error. If $\mathrm{n} / \mathrm{N}$ is likely to be large and sampling is without replacement the estimate of $n$ must be adjusted.

$$
\mathrm{n}_{\mathrm{a}}=\frac{\mathrm{n}}{1+\mathrm{n} / \mathrm{N}}
$$

Simple random sampling is a very simple method which provides unbiased estimates with calculable precision. It is most useful for initial surveys or for sampling from small completely enumerated populations. The irregular 
distribution of the sample units is a marked disadvantage when it is desired to construct maps from the results of the sample, in addition to obtaining estimates, for example in soil surveys, surveys of natural regeneration or vegetation, etc.

\subsection{Stratified random sampling}

In this method the population is divided into a number of divisions or strata (analogous to blocks). Within these strata the samples are taken at random using simple random sampling.

The strata are chosen in such a way that the variability of the sampling units within a stratum is less than the variability between strata. This improves the precision of the estimates provided that stratification has been successful. Effective stratification therefore depends on some prior knowledge of the population. For example in an inventory of a large forest the total area could be divided into strata of similar species and ageclass, and a number of sample plots measured at random points within each stratum.

Stratification may be administratively desirable since estimates of the mean are required for each subdivision. The strata may be the same size or of differing size, and, as for blocks, need not be contiguous.

In the analysis the means and variances for the individual strata are first calculated and then these estimates are combined to give estimates of the population mean and standard error as in the following procedure: -

(i) Estimate mean for each block.

(ii) Estimate total for each block by multiplying the block mean by the block size.

(iii) Sum the block totals to obtain estimate of overall total.

(iv) Divide overall total by by total size to obtain estimate of overall mean.

For the variance of the overall mean the same procedure is followed except that the block variances are multiplied by the square of the block size and the total variance divided by the square of the total size. The formulae given above for simple random samples are used to compute the block variances. The finite population correction should be applied to the block variances.

Sampling error and confidence limits are calculated as above but the degrees of freedom depend on the homogeneity of the block variances. If these are homogenous the total degrees of freedom can be taken as the sum of the block degrees of freedom. Otherwise the total degrees of freedom may be considerably reduced (see Cochran, 1953, section 5.4). 


\section{(a) Allocation of sampling units}

Given a total sample size of $n$ units, how are these units allocated to the different strata? There are basically two methods:-

\section{(a) Proportional allocation}

The sample units are allocated in proportion to the size of the stratum: in a stratum having $2 / 5$ of the total number of units in the population one would allocate $2 / 5$ of the $n$ sample units. The number of sample units in the $i^{\text {th }}$ stratum $\left(n_{i}\right)$ is given by

$$
n_{i}=\frac{\left(N_{i}\right)}{(N)} n
$$

where $N_{i}$ is the total number of units in the stratum and $N$ is the total number in the population.

The sampling fraction is thus the same in each stratum.

\section{(b) Optimum allocation}

In this method an attempt is made to obtain the smallest standard error (for the population mean) possible with a total sample size of $n$. The allocation is given by

$$
n_{i}=\frac{\left(N_{i} s_{i}\right)}{\left(\sum N_{i} s_{i}\right)} n
$$

where $s_{i}$ are estimates of the within stratum standard deviations.

Allocation is thus on the basis of the within stratum variability and requires prior knowledge of this variability which may not be available. As a refinement, if sampling costs vary from one stratum to another, a cost factor may be introduced to give an allocation providing the most information per unit of money.

\section{(b) Sample size}

An estimate of the total sample size ( $n$ ) needed in a stratified random sample may be obtained from the following formulae:-

for proportional allocation

$$
n=\frac{N \sum N_{i} s_{i}{ }^{2}}{N^{2} E^{2}+\sum N_{i} s_{i}^{2}}
$$

for optimum allocation

$$
n=\frac{\left(\sum N_{i} s_{i}\right)^{2}}{N^{2} E^{2}+\sum N_{i} s_{i}^{2}}
$$


where $E$ is the desired standard error of the population mean. When the sampling fractions $\left(n_{i} / N_{i}\right)$ are likely to be very small for all strata or when sampling will be with replacement, the second term of the dengminators of the above formulae $\left(\mathrm{N}_{i} \mathrm{~s}_{i}{ }^{2}\right)$ may be omitted leaving only $\mathrm{N}^{2} \mathrm{E}^{2}$.

\section{(c) Advantages and disadvantages}

Stratified random sampling is one of the most useful methods available for estimating population characteristics. It is extremely flexible since the number of samples in any stratum can be adjusted according to the variability of the stratum, or according to the value of that stratum to the object of the survey. Hence a minimum of work is required in those strata of little importance. The method provides separate estimates of the mean and variance of each stratum which may be an important part of the objects of the survey.

For a given sampling fraction the method often provides more precise estimates of the population parameters than would be provided by a simple random sample of the same size.

A disadvantage is that the strata sizes must be known and that some prior knowledge of the population is essential for efficient stratification. Computation, of the precision of the estimates can involve considerable work if the strata sizes and variances are very different.

\subsection{Systematic sampling}

In systematic sampling the units are not selected at random but according to a pre-determined pattern. Often the only element of randomness is the starting point of the pattern. The most common pattern, occurring in the field, is a grid having the sample units located at the intersection of rectangular co-ordinates. Another example is the selection of every $n^{\text {th }}$ unit from a list of $N$ units.

Systematic sampling has been widely applied in forestry for two main reasons. Firstly, the location of sample units in the field is often easier and cheaper and, secondly, due to an intuitive feeling that a sample spread over the entire population will bemore representative, and hence more precise, than a random sample. The first reason is often true and, in addition, a systematic layout by covering the whole area, assists in mapping or in demarcating boundaries. The second reason may be true; howeve:, due to the non-random element the precision can only be calculated approximately (the common procedure being to use random sampling formulae, although many formulae applicable to special circumstances have been developed).

There is a danger of systematic bias if the sampling interval coincides with some periodic variation in the population. Such a periodic trend may be caused by geographical, geological, biological features, or by human activities. For example if the sampling interval coincides with alternating ridges and valleys a biased result will occur if the variable(s) being measured are affected. This is illustrated below:- 


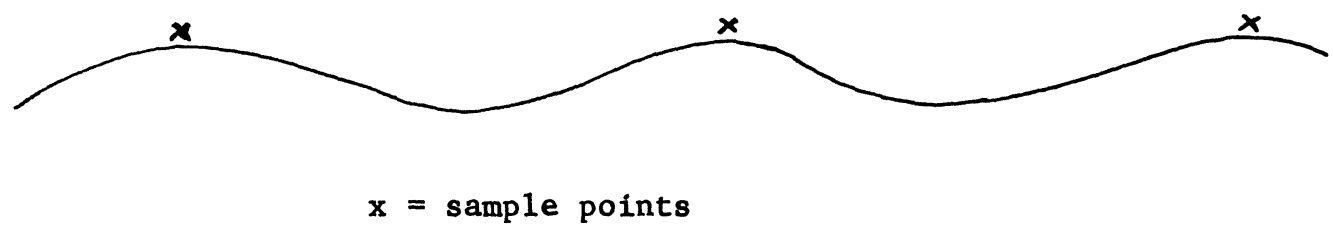

Systematic sampling is thus a useful objective method of sampling provided periodicities are unlikely to occur. From a practical point of view the regular spacing of the units at predetermined intervals may save time and money in forests where access and transport are major problems. Experience has shown that in a few cases the results from systematic sampling will be very misleading, but that most of them will give estimates having a precision as good as, or slightly better than, that shown by random sampling formulae.

\subsection{Multi-stage sampling (sub-sampling)}

If it is difficult or expensive to locate a sample unit but relatively easy or cheap to measure the unit, it would seem logical to measure several units at or near each location. For example, in a survey of fungal attack on the leaves of trees in a provenance trial with large plots it would be possible to take a random sample of 50 leaves from each provenance in the trial. However, this would necessitate counting and identifying all the leaves which is a virtually impossible job; a more logical method would first be to select five trees at random in each plot and then to select ten leaves on each tree. One is now concerned only with the leaves on each of the ten selected trees. This is known as a two-stage sample; the first stage is the selection of the primary units - the trees, whilst the second stage is the taking of a sub-sample of secondary units - the leaves - from each of the chosen primary units. The selection of the samples should be at random in each stage. This type of sampling is illustrated in Figure 6.1 .

Replication

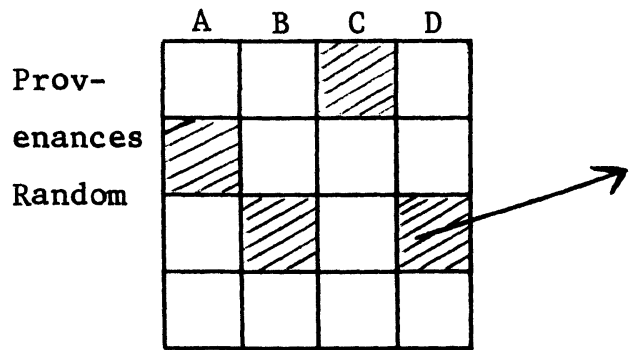

primary units

(e..g. provenance plots)

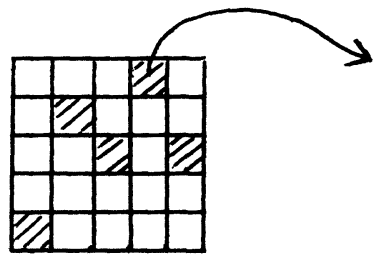

secondary units

(e.g. trees)

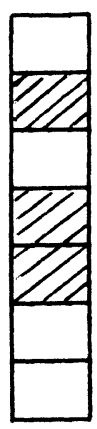

tertiary units (e.g. leaves)

Figure 6.1 Multi-stage sampling

An advantage of this method of sampling is that it gives the sampler greater scope since he can choose the sizes of the samples in each stage. An important result of the analysis is an estimate of the variation in the different stages. In the example given above one can estimate the component 
of variance between trees and the component between leaves within trees. This information enables one to plan the size of the samples required. A general property of multi-stage samples is that the same standard error of the sample mean can be attained by using various combinations of sample sizes. Which combination is best will depend on the objectives of the sampler. In order to determine sample size it is usually necessary to carry out a small preliminary sample unless information is available on the variance components.

For a fixed number of sample observations, multi-stage sampling is usually less precise than simple random sampling. The advantage of the method is that by reducing the cost per observation it allows one to obtain the desired precision at a lower cost. Usually the precision and cost both increase as the number of primaries is increased and the number of secondaries decreased. Computation of the analysis is not difficult particularly if the units in any stage are the same size. The analysis of the sample data can be presented as an analysis of variance. In sampling for wood properties a multi-stage design is frequently used with, for example, a sites as the first stage, b trees per site as the second stage and $c$ samples per tree as the third stage. This would give the following ana'ysis of variance:-

\begin{tabular}{|l|c|c|c|}
\hline \multicolumn{1}{|c|}{ Variation due to } & $\begin{array}{c}\text { Degrees } \\
\text { of freedom }\end{array}$ & $\begin{array}{c}\text { Mean } \\
\text { square }\end{array}$ & $\begin{array}{c}\text { Variance } \\
\text { components }\end{array}$ \\
\hline Between sites & $a-1$ & $\mathrm{MS}_{1}$ & $\mathrm{~s}_{\mathrm{c}}^{2}+\mathrm{cs} \mathrm{b}_{\mathrm{b}}^{2}+\mathrm{cbs} \mathrm{s}_{\mathrm{a}}^{2}$ \\
Between trees within a site & $\mathrm{a}(\mathrm{b}-1)$ & $\mathrm{MS}_{2}$ & $\mathrm{~s}_{\mathrm{c}}^{2}+\mathrm{cs} \mathrm{b}_{\mathrm{b}}$ \\
Between samples within a tree & $\mathrm{ab}(\mathrm{c}-1)$ & $\mathrm{MS}_{3}$ & $\mathrm{~s}_{\mathrm{c}}^{2}$ \\
\hline TOTAL & $\mathrm{abc}-1$ & & \\
\hline
\end{tabular}

The confidence limits for the overall mean $(\bar{x})$ are easy to compute since:-

$$
s_{\frac{2}{x}}=\frac{M S_{1}}{a b c}
$$

with (a - 1) degrees of freedom. Estimation of the variance components is straightforward and enables one to predict the variance of the sample mean for differing values of $a, b$ and $c$, since:-

$$
s_{x}^{2}=\frac{s_{a}^{2}}{a}+\frac{s_{b}^{2}}{a b}+\frac{s_{c}^{2}}{a b c}
$$

\subsection{Sampling for proportions}

It is often necessary to estimate the proportion or percentage of units in a population which possess some characteristic or fall into some defined class (e.g. alive or dead; infected or healthy; germinated or ungerminated). Data of this type are likely to follow the binomial distribution and therefore require slightly different statistical techniques. 


\subsection{Simple random sampling for proportions}

If a simple random sample is taken from a population then the estimate of the proportion, $p$, having the specified characteristics is

$$
\mathrm{p}=\frac{\text { number having the characteristic }}{\text { number observed }}
$$

The estimated standard error of $p$ is

$$
s_{p}=\frac{p(1-p)}{(n-1)}\left(1-\frac{n}{N}\right)
$$

where $\mathrm{n}$ is the number of units in the sample, and $\mathrm{N}$ is the total number of units in the population. If $\mathrm{N}$ is very large relative to $\mathrm{n}$ then the finite population correction can be ignored.

For certain sample sizes $(n=10,15,20,30,50,100,250,1000)$ confidence limits can be read directly from tables given in Freese (1962) and Snedecor and Cochran (1967). These tables can also be used to estimate the sample size needed to obtain a given confidence interval.

A normal approximation to the 95 percent confidence limits can be computed as

$$
p \pm\left[s_{p}+1 / 2 n\right]
$$

where $t$ is Student's $t$ for $(n-1)$ degrees of freedom and at the 95 percent level. This approximation is only valid under certain conditions given in the table below:-

\begin{tabular}{c} 
Value of $\mathrm{p}$ \\
\hline 0.5 \\
0.4 \\
0.3 \\
0.2 \\
0.1 \\
0.05
\end{tabular}
np must be greater or equal to

If it is likely that a sample of over 100 will be needed sample size can be estimated by

$$
n=\frac{4 p\left(\frac{1}{2}-p\right)}{E^{2}}
$$

for 95 percent confidence

where $\mathrm{E}$ is the precision with which $\mathrm{p}$ is to be estimated (expressed in the same form as $p$, either percent or decimal).

A value of $p$ of 0.5 will give the maximum value of $n$; consequently if one has no idea of the value of $p$ one should use a value as close to 0.5 as might reasonably occur. 


\subsection{Cluster sampling for proportions}

In sampling for proportions the cost of selecting and locating an individual is normally very high relative to the cost of determining whether or not the individual has a certain characteristic. In cluster sampling a group of individuals becomes the unit of observation and the unit value is the proportion of individuals in the group having the specified characteristic.

For example, the germination percent of a batch of seed might be estimated by the use of clusters. The advantage of clusters is that some of the hazards of germination tests may be avoided. If all the seeds are in a single dish any mishaps (fungal attack or drying out) may affect the entire test. It is normal to place a fixed number of seeds (100 or 200) in each of several dishes. Another example is the selection of plots of trees rather than individual trees when assessing for survival or disease infection.

If the clusters are large enough (over 100 individuals per cluster) and approximately the same size the procedures used in Section 6.221 for simple random sampling of measurement data can be used for computing means and standard errors. The value of $n$ is thus the number of clusters and not the number of individuals within a cluster.

If the clusters are small (less than 100 individuals per cluster) or some of the observed percentages are greater than 80 or less than 20 , it is wise to transform the percentages before computing means and standard errors. The appropriate transformation is the arcsin square root percent, tables of which occur in most statistical text books.

Any of the designs given in Section 6.22 may be used for cluster sampling of proportions. In place of individuals the clusters become the units of which the population is composed. Stratified random sampling could be used to estimate the germination percent of a seed lot made up of seed from several sources. The sources become the strata, each of which is sampled by two or more randomly selected clusters of 100 seeds.

\subsection{Sampling of count variables}

Data such as the number of cankers on a tree, the number of bends on the bole of a tree, and similar count variables having no fixed upper limit can cause problems when they are analysed since they tend to follow non-normal distributions. This is especially true with small counts and those with numerous zeros.

This problem is best overcome by defining the sample units so that most of the counts are large and to take samples of 30 units or more. The normal distribution methods can then be used for estimating the mean and standard error.

\subsection{Classification data}

The observations in a sample may be classified according to two or more characteristics. A tree can be classified as to its position in the 
canopy and at the same time as to whether it is diseased or not. One could classify by straightness of stem and site. Given such a two-way (or even three-way) classification one may wish to know whether the classification of an individual by one system is independent of its classification by the other. In other words is the straightness of stem dependent on the site.

The hypothesis that two or more systems of classification are independent is tested using the chi-square statistic. Details of the test and tables of chi-square are given in most text books on statistics.

\subsection{Shape and size of sampling units}

In finite populations the choice of the shape and size of the units is usually determined by the nature of the population. For example, in estimating the mean height of trees in a compartment, each tree is a unit which may or may not be included in the sample. Similarly in sampling from a batch of seeds, the individual seed is the sampling unit. In infinite populations, however, the shape and size of the units can profoundly affect the efficiency of the survey. The optimum size and shape should give the highest precision at the lowest cost.

\subsection{Shape}

The shape is frequently dictated by practical considerations of the difficulty of demarcating and laying out the unit. As far as possible the shape of the units should correspond to some naturally occurring shape, for example, a line of transplants or a group of trees rather than to an artificial unit imposed on the population. A unit which gives a short outside edge is preferable to one which gives a long outside edge since it reduces the number of edge trees. Thus, a circular field plot is preferable to a square one, and a square one is preferable to a rectangular one.

\subsection{Size}

In general, the statistical efficiency of a sample is increased by decreasing the size of the sampling units and increasing the number of units measured. The smaller size of the units may, however, increase the cost of the sample by making the measurements more difficult and by increasing the travelling time and other work. In addition a very small unit may increase the area of the plot which is subject to edge effects. Units of 1 square metre are commonly used for surveys of numbers or heights of seedlings, or ground vegetation, whilst units of $0.02-0.1$ ha are commonly used for surveys of numbers, or volumes, of trees in established plantations. A good general rule is to use the smallest sampling unit which does not introduce severe practical problems.

\subsection{Grouth plots}

Information on the growth and yield of a species is essential once it is known that the species can be established. Much of this information will come from species trials which are left to grow on until rotation age. However, it is usually the case that a promising species is planted on a 
large scale before any final assessments of yield are available. It is important that this type of planting is monitored for growth and yield. Too often large areas of plantations have been established, usually with exotic species, with no planning for the collection of the necessary data on growth, with the result that the research worker and the manager do not have the information that they need.

Two important points are the use of exotics and the fact that the plantations may cover large areas embracing a wide range of site conditions. The use of exotics means that virtually no information will exist concerning the long-term performance of that species. The optimum silvicultural treatment for specific end results will not be known. Where the species has been or is to be planted on a large scale there may be very different site conditions from one plantation to another resulting in very different rates of growth.

Growth plots may be temporary or permanent; that is measured once only or re-measured after an interval of time. Temporary plots are essentially an inventory tool and unless analysed in conjunction with increment borings or stem analyses have a very different purpose from that of permanent growth plots. known on:-

Permanent growth plots provide the most reliable source of information

(a) how forest stands are changing with time

(b) how stands vary with site and treatment

(c) the relations between stand parameters (e.g. stand heights, basal area, commercial volume, etc.) and of individual tree parameters (height, density, form, wood quality and value, etc.) with stand density.

Permanent growth plots in industrial scale plantings should be located in random positions and if possible installed before the time of first thinning or other silvicultural treatment. It is possible that the plots may form part of a continuous inventory organised by the forest management branch. A "Guide to the Management of Permanent Sample Plots" is given in Appendix 10. This is intended for continuous forest inventory work but the details on location of plots, laying-out, recording etc. are equally applicable to permanent plots used for sampling extensive plantations.

For long term plots where the effects of specific silvicultural treatments on stand and tree parameters are studied in designed experiments, Appendix 10 is also applicable as regards laying out, recording and assessing the plots. 
Programmes for the study of wood quality in relation to afforestation schemes need to be developed systematically, by stages as follows:-

(1) A review of existing forest resources.

(2) A survey of present demand and projected future demand for wood.

(3) An evaluation of the extent to which the current and the projected demand can be met by management of existing forest resources, in terms of:-

(a) quantity of wood,

(b) quality requirements of the wood using industries.

(4) A plan to make the best use of existing resources to meet both current requirements and anticipated future demand.

(5) An assessment of the necessity for afforestation to increase the production of wood to meet anticipated future demand, in terms of: -

(a) quantity

(b) quality.

(6) A plan for the research required to investigate wood quality in afforestation schemes, and the possibilities for substantial improvement in quality, including:-

(a) selection of the species and provenances most suitable for afforestation and to meet the anticipated demand, both in terms of quality and quantity

(b) appropriate silvicultural management to obtain the maximum yields of wood of an acceptable quality,

(c) tree breeding to improve both yields and quality.

(7) A development plan for afforestation, in which the possibilities for improvement of wood quality are taken into account.

This Section is concerned largely with some of the procedures that are required in Stage 6 of this programme.

6.31 A plan for the research required to investigate wood quality and the possibilities of substantial improvement in wood quality in afforestation schemes

The term quality has meaning only in relation to a specified end use. Because wood has many different uses, an assessment of the wood quality of a species or provenance, or even of an individual tree, may involve the consideration of a large number of physical and mechanical properties and other characteristics.

Knowledge of the following wood properties is desirable, so that the full potential for the utilization of a species or provenance can be assessed: -

(1) colour and general appearance

(2) density

(3) ring pattern and structure

(4) strength (normally strength in bending, axial compression, hardness, shear and cleavage, although information on impact bending, tension and lateral compression strength may be required for some species and for certain applications) 
(5) grain inclination

(6) shrinkage (radial, tangential, and longitudinal)

(7) behaviour in seasoning (air drying and kiln drying)

(8) natural durability

(9) permeability to preservative treatment

(10) wood working (sawing, planing, boring, turning, screw and nail holding and resistance to splitting when nailed)

(11) glueing

(12) peeling on a lathe

(13) slicing

(14) pulp and paper making characteristics.

It is also desirable to know something of the chemical composition of the substances in the wood, including the content of cellulose, lignans, gums, resins and tannins.

Some wood properties are associated or interdependent, e.g. most strength properties, except cleavage, have a relationship with density, and natural durability is connected with the type and amount of chemical substances deposited in the cells. Similarly, seasoning properties are related to the shrinkage in different directions in a piece of wood and to the angle of grain in relation to the length.

Although such interrelationships are recognised, they have rarely been defined with sufficient precision to be able to predict, very accurately and consistently, one particular property from a knowledge of others. In general, the properties and use characteristics of a wood can be defined satisfactorily only by direct tests but, since such tests are costly, they can be applied only on a limited scale. Moreover, they usually involve destructive sampling and are impractical for such purposes as, for example, the assessment of the wood quality of candidate trees in a breeding programme.

\subsection{The use of key index features as indicators of wood quality}

Certain wood features are good general indicators of wood properties and uses. Wood density is outstanding in this respect since it not only influences strength but also shrinkage, seasoning, woodworking, glueing, slicing, peeling and paper making.

The key features are those which are either closely related to other properties and can, therefore, be used as indicators, or in which a small amount of variation influences considerably the usefulness of the wood for certain end uses. These include:-

(1) colour and appearance

(2) ring width and structure (proportion of latewood, proportions of different tissues, reaction wood)

(3) wood density

(4) grain angle

(5) shrinkage

(6) cell dimensions. 


\subsection{Direct testing of wood properties and use characteristics}

The measurement of these key features will usually give a good indication of the possible uses for the timber but it should be recognized that density, for example, is only moderately well correlated with some strength properties, the relationship of shrinkage with seasoning is not very precisely defined, and woodworking characteristics are often difficult to predict from other properties. Consequently, there are situations, at various stages of species and provenance research, where direct testing of certain wood properties must be applied.

This may be necessary, in the first instance, for the evaluation of some provenances in the natural range for which little or no information on wood characteristics is at present available. Some direct testing may also be required at an early stage in research programmes, to establish more definitely the relationships between structural features or properties and use characteristics on which the use of wood structure as an index of wood quality depends. This may be necessary, for example, to set the standards by which elite trees are selected for a breeding programme, i. to establish the relationships between end use properties and wood structure features from which standing trees can be compared by small scale non-destructive sampling. In afforestation schemes, laboratory and semi-commercial scale direct testing may also be required at a later stage to give reliable information, particularly with exotic species, on which to base decisions for investment in processing equipment.

Where it is desirable to compare wood features or properties with other tree parameters such as height, diameter, volume, stem and crown characteristics, it is necessary to record these features carefully when sampling the trees. Standard techniques for doing this are given in the suggested procedures for sampling and for recording field data (Special Appendix 1 , not included in this Manual but obtainable separately from the Commonwealth Forestry Institute, Oxford).

6.34 Suggested procedures for the evaluation of wood quality in relation to species and provenance research

Usually it is neither necessary nor desirable to attempt a full range of wood quality tests on a tree species or provenance at the start of a trial programme, and even when little is known about a species it is best to carry out tests in a series of stages. These can be identified as follows:-

(1) Pilot tests.

(2) General utilization tests.

(3) Tests designed to study variation in properties, to derive information for purposes of silvicultural management, and in particular to assess the effect of heritable factors (e.g. provenance, progeny) and of site conditions.

(4) Specialized tests e.g. to establish relationships between key index features and other features, properties and use characteristics.

The circumstances in which the various stages of wood tests are 
applicable in species and provenance research are discussed below and the general requirements for such tests are given in outline. Detailed procedures for field sampling, laboratory examination of samples, analysis of data and evaluation of results are given in the Special Appendices (obtainable separately from the Commonwealth Forestry Institute, Oxford) and in the references cited.

It should be remembered in all tests, and in particular when examining plantation grown material, that valid comparisons can be made only between trees of the same age using comparable parts of the tree.

\subsection{Pilot tests}

These are limited or exploratory tests, designed to give information quickly on the properties of a species, as a guide to the simple utilization of wood, sawn or in the round. They are particularly applicable to the study of an unknown species just coming on to the market from a natural forest but would also be useful for an introduced species about which nothing is known of the wood properties of trees grown in that country.

\section{Suggested test procedures}

(i) Sampling systems

No attempt is made to obtain a statistically valid sample, although enough material should be collected to cover the main range of sites and tree sizes. Often these are found in arboreta and other small introductory plantations. A convenient way to collect samples is to select some quarter sawn and some flat sawn timber from sawmills in each locality.

(ii) Laboratory measurements

The samples of timbers for pilot tests should be used to examine the key wood features and also possibly to obtain some values for the main strength properties such as static bending, axial compression and hardness. Whenever possible, tests should be conducted according to the standard procedures for general utilization tests described below.

(iii) Analysis of test results

Mean values for density, strength and shrinkage will give a good general idea of the physical and mechanical properties of the species, particularly if samples are large enough to calculate standard errors of the means, and these tests can normally be carried out within a few weeks. Where sufficient field plots exist, samples may be taken from different varieties or provenances of a species.

\subsection{General utilization tests}

These are tests designed to obtain average values for wood properties, and to ascertain the extent of variation in properties to be expected from the range of major sites on which the species is growing, without 
attempting to study in detail within-tree variation or to relate properties to site characteristics. The objective is to get as reliable an average value for the property as is possible for each site, within the limitations of time and money available to carry out the test. A modified form of the sampling strategy suggested by Pearson (1969) is recommended here.

\section{Suggested test procedures}

\section{(i) Sampling systems}

Tests should be planned so that statistical analysis of the results can be carried out. It is suggested that the following sampling system should be used in the first instance for density, strength properties, and shrinkage, until sufficient information is available to determine an optimum sampling strategy with an acceptable degree of precision:-

(a) $n$ sites (depending on the number of identifiable sites)

(b) 5 to 10 trees per site chosen at random

(c) 1 log per tree chosen at random from the merchantable bole of the tree and of sufficient size to give, when sawn up, the pieces required for all tests. (Logs of at least $4.2 \mathrm{~m}$ in length are required, and if air-drying and woodworking tests are to be carried out it will usually be necessary to take a second $\log$ from each tree)

(d) 4 samples to be taken for density and strength tests per radial board at $0.2,0.4,0.6$ and 0.8 of the distance from pith to bark.

The sample may be stratified over different varieties or provenances; further, such samples could be selected in well designed field provenance trials (see Chapters 4 and 5). A suggested procedure for sampling and for recording field data in general utilization tests of wood properties is given in Special Appendix 1.

\section{(ii) Laboratory measurements}

A general utilization test may include the full range of wood properties 1 isted in Section 6.31 but very often a preliminary test is carried out in which physical properties, mechanical properties and wood working characteristics only are examined in the first instance. It is emphasized that the range of tests and the procedures recommended here are not intended to constitute a comprehensive system for testing wood properties and use characteristics. The pur pose is to obtain information to an extent that will define the general usefulness of the species and to indicate the desirability of further testing of the wood for particular purposes.

Procedures that are applicable in general utilization tests are described in outline in Special Appendix 2 and in greater detail in the following references. Lavers (1969), BSI (1957), Pearson and Stevens (1929), Redding (1958) and Knight (1956).

\section{(iii) Analyses of test results}

Some species show considerable within-tree variation in properties, 
particularly from the pith outwards. Consequently, a value calculated just from the mean of values of test pieces taken from the pith outwards may give a very misleading estimate of the "average" values for such important properties as density and strength. The results from the tests carried out at the $0.2 \ldots .0 .8$ radius positions should therefore be weighted according to the quantity of wood represented by each position, i.e. the 0.2 radius position should be multiplied by 1 , the 0.4 by 4 , the 0.6 by 9 , and the 0.8 by 16 and the total divided by 30 (the sum of $1,4,9$ and 16). This gives a weighted mean property for each tree.

By an analysis of variance of the test data, the amount of variation due to sites, to trees within sites, and positions within trees can be determined. Standard errors of the mean and confidence limits can be calculated and, by using variance components, alternative sampling techniques can be derived to achieve the required precision for estimation of the mean, if the initial sample is shown to be inadequate. (The $95 \%$ lower confidence limit of the mean for strength properties is frequently used by engineers requiring timber for structural purposes.)

6.343 Tests designed to study variation in properties, particularly in relation to the effects of genetic factors and of site conditions

The variation in some important properties such as density, cell dimensions, strength, shrinkage and grain angle is often large within individual trees and between the average values for different trees of the same species or provenance. If a study of key index features, or a general utilization test as outlined above, shows that there is considerable variation in wood characteristics and properties, it may be necessary, in connection with species and provenance trials, to carry out further investigations, with one or more of the following objectives:-

(1) To estimate the range of variation in structural features and the corresponding variation of properties and use characteristics of the species.

(2) To describe and to define within-tree patterns of variation in wood structure, and the extent to which within-tree patterns vary in different trees, as an indication of the relative importance of within-tree, between-tree and between-provenance variability in wood properties.

(3) To determine the influence on wood structure and on patterns of structural development, of age, rate of growth, height, diameter, stem form, crown size and shape, and of their interactions.

(4) To examine the relationships of structural features, measured from small scale sampling in the lower bole, with estimates of whole tree values for these features. The purpose is to see if between-tree differences in wood quality, and the influence of such factors as age, rate of growth, height, diameter, stem form, crown size and shape and their interactions, can be estimated satisfactorily by comparison of limited numbers of samples of small size taken at convenient heights.

(5) To compare the extent and patterns of variation in structural features of trees from different sites, for the purpose of estimating the influence on wood quality of site factors, including biotic, climatic and edaphic factors, and silvicultural treatments. 
(6) To make estimates of the extent of between-tree variability in structural features that is due to genetic differences, as a guide to the possibility of selecting and breeding from trees of superior wood quality.

\section{(a) The effects of genetic factors and of environmental conditions on wood quality}

In species and provenance research, the reasons for investigation of variation in wood structure are commonly concerned with tree improvement, either by selection of the most suitable species for each site, or by control of silvicultural conditions, or by selection and breeding. A replicated provenance trial is designed primarily to separate genetic and environmental (replicate) effects at the population level.

Further study, either of genetic or of environmental effects, on wood quality may be warranted:-

(i) where there are two or more recognized varieties of a species,

(ii) where a species has a wide geographical distribution in its natural range, or occupies a wide range of site types,

(iii) where a species has been established successfully as an exotic over a wide range of geographical locations and site types,

(iv) where considerable variation in growth rate has been found between trees from the same location or between mean values for growth rates at different localities.

Several tropical pine species, especially Pinus caribaea, provide examples of these requirements.

From an analysis of variance of the data from pilot tests or from general utilization tests, of the type described in Sections 6.341 and 6.342 , it will usually be apparent if the between-site or between-plot differences that could be associated with environmental conditions or treatments, or the between-tree (within-plot) differences that are 1ikely to be associated with genetic factors, are of such an order that further investigation should be considered.

\section{(b) The relative importance of genetic and environmental factors}

The overall extent of the variation in wood properties of a species or provenance can be determined fairly accurately from pilot tests and general utilization tests, and will give a general indication of the possibilities for improvement of wood quality. The relative importance of the genetic factors and of the environmental factors in determining this variation should also be known, so that the correct emphasis can be given to site selection, silvicultural treatments, or breeding in framing a silvicultural policy for tree improvement. This information, however, can be obtained with certainty only from carefully designed clonal and progeny trials since part of the between-tree variation in any characteristic may be due to micro-environmental differences.

Because of the complex interactions between the genetically controlled growth characteristics of a tree and the biotic, climatic and edaphic con- 
ditions of the place where it grows, determination of the precise effects and relative importance of either the heritable or the environmental group of factors on tree growth and wood quality is an extremely difficult matter. Clonal and progeny trials for this purpose will require considerable facilities in the field and in the laboratory and substantial deployment of skilled manpower. However, at the population level, comparisons are more straightforward provided that population means are calculated from sufficient numbers of trees to characterize all the variation present.

It should be recognized that, whilst for some characteristics examination of juvenile properties may give a satisfactory indication of later performance, a valid assessment of the variation in wood properties may not be obtainable until trial plots are at least beyond the "juvenile wood" stage. Furthermore, since analysis of the results of such trials gives an assessment of the relative importance of genetics and environment only for the particular population or individual genotypes and sites examined, they may not be applicable in other situations. Again, although the overall extent of variation in growth in wood properties attributable to the effect of sites can be defined in this way, it may be extremely difficult or impossible to identify and to isolate the separate effects of the various factors involved, including some major features of climate and soil. Techniques of multiple regression analysis are as applicable for wood properties as for growth (Section 6.137).

Where differences in growth and in wood properties between varieties or provenances of the same species are known to be considerable, the management decision likely to be of greatest urgency is to select the variety that will give the highest yield of wood of acceptable quality in each of the sites available for planting. In these circumstances priority should be given to trials of the varieties and provenances that are most promising (Sections 1.2 and 5. ), and for which seed is available (Section 2.3 ), conducted over a wide range of potential sites. International provenance trials, of which several have recently been established in the tropics, will, in due course, provide material suitable for making a good assessment of the overall effects of the interaction of different seed origins and of site conditions using genotype-environment interaction analysis (Section 6.138).

(c) Relationships between wood properties in the juvenile stage and mature whole tree values

It may be possible to establish relationships between the values for some features and properties of wood in the juvenile stage and the wood formed later or with values for mature whole trees. This would enable an earlier comparison of the performance of different species, provenances and progenies or an earlier study of site effects and management treatments.

To investigate relationships between juvenile wood and outer wood requires the detailed examination of within-tree variation of a large number of trees. The cost will be considerable, and it should be recognized that for some properties it may not be possible to derive sufficiently precise predictive equations. 
If, however, satisfactory relationships of this sort could be established, there would be a substantial saving of time, e.g. in choosing provenances and in selective breeding programmes. It is therefore suggested, when projects for the examination of within-tree patterns of variation are considered, that the sampling plan and laboratory procedures should be designed to give the maximum amount of information for all purposes for which a knowledge of this feature of growth and wood structure is important, including the prospects for future improvement as well as for immediate utilization.

(d) Inter-relationships between structural features, properties and use characteristics of wood

If it can be demonstrated that there are close and definable relationships between some of the structural features or simple physical properties of wood, that are easily measured, and the more complex properties or end use characteristics, which usually need to be assessed by laborious and costly direct testing, then the investigation of patterns of variation in wood, and of the factors that influence them, will be greatly simplified. In particular, it would be very advantageous if the wood structure and physical properties, to be examined in such projects, can be restricted to some of the key features listed in Section 6.32, that can be measured accurately and rapidly on small specimens, and often with non-destructive sampling. It should then be possible to plan for an adequate scale of sampling and at the same time to keep laboratory work and analysis at an attainable level.

\section{Suggested test procedures}

In general, forest managers will be concerned to know the effects of genetic factors or of environmental conditions on the following:-

(1) the extent and pattern of within-tree variation in wood properties

(2) whole tree mean values for wood properties

(3) mean values per hectare.

The procedures described here are designed accordingly.

\section{(i) Sampling}

It is neither possible nor advisable to attempt to prescribe a standard sampling system that can be guaranteed to meet all requirements. The preliminary sampling scheme put forward here has given satisfactory results when applied to a range of species and locations. It has the dual merit that it is not often likely to lead to excessive over-sampling and it provides a basis from which a further sampling scheme can be derived to obtain a stated degree of precision with minimum effort.

If the intention is to estimate the range of variation in a wood property, or to compare different provenances, whether in exotic plantations or in natural forest, then samples should be taken from all the major site types that can be defined for each provenance or origin. Information to be recorded for the description of the environmental conditions (climate, soil, topography and vegetation) at a site, is given 
in Special Appendix 1, and Chapter 3.

For each species or provenance in each site type, a minimum of two plots should be selected, so that an estimate can be made of the extent of the variation in growth and wood properties occurring within what is considered to be a distinguishable unit of land (see also Section 3.2). This information on between-plot variation may give some indication of the uniformity of the site type, or of the reaction of the species to comparatively small changes in site conditions. Suggestions for the selection and demarcation of sample plots at each site type are given in Special Appendix 1.

From each plot, a minimum of five randomly chosen trees should be sampled, and whenever possible ten trees should be taken. If the species is very variable, it may be advisable to take a much larger sample, but this should not be done until a preliminary sample of five or ten trees has been examined and an optimum sampling strategy determined. When the first sample is taken, a plot should be permanently demarcated, of adequate size so that a larger sample can be obtained later, if necessary. For all trees sampled, a record should be made of the exact location of the tree, of the plot in which it occurs, and of its size and form, according to the procedure given in Special Appendix 1.

Where there is considerable variation within trial plots or larger plantations of a given species or provenance in the age or size or form of trees, it may be advisable to stratify the sample by age classes, diameter classes or crown classes according to the situation.

In the preliminary sampling of any species or provenance, whole trees should be taken so that billets or discs can be removed at frequent fixed intervals of the height, for example at $10 \%$ intervals or at fixed growth internodes, as described in Special Appendix 1. For subsequent sampling, it may be possible to reduce the number of billets taken within trees, and four positions have been found to be adequate to describe variation with height in most species - for example at $5 \%, 15 \%, 35 \%$ and $65 \%$ of total height. In some circumstances, and where satisfactory relationships have been established previously between small samples and whole tree values, it may be possible to carry out the greater part of any further sampling from borings taken in the lower bole.

Investigations carried out at the Commonwealth Forestry Institute, Oxford, especially with several fast growing tropical species, have shown that variation around the circumference is usually small, compared with other sources of variation (e. $g$. distance from the pith or between-tree differences), except in trees with fluted boles or with considerable development of reaction wood. Two radial positions, preferably taken at random, or where this is not possible a complete diameter, will usually constitute a satisfactory preliminary sampling system, at any height level in the tree.

In choosing a system for sampling to examine within-tree variation, it should be recognised that variation from the centre of the stem to the outside is normally greater than from the bottom to the top of the tree. 
It is therefore advisable, in the first instance, to sample more intensively along the radius than axially. The choice of sampling interval will depend upon the type of test undertaken, the size of specimen required for examination and the accuracy that is expected. Density, for example, when measured by optical scanning of radiographs, can be recorded at intervals of $5 \mu$ or less, but a very satisfactory estimate, of both within-ring variation and mean values for whole rings, can be obtained at measurement intervals of $100 \mu$ to $200 \mu$ or more. For fibre length and other fibre dimensions, sampling at every second or fourth annual ring may be required to establish the general trends from pith outwards, but measurement at much wider intervals may be satisfactory when general patterns are known.

\section{(ii) Laboratory measurements}

Direct test procedures to determine values for wood properties and use characteristics in the investigation of within-tree and between-tree variation will usually be similar to those proposed for the standard general utilization tests described in Special Appendix 2.

For the use of the key index features, 1isted in Section 6.32 there are, as yet, no recognized standard test procedures, although this approach to the examination of variation in wood quality is now used frequently by research laboratories. Some of the methods developed at the Commonwealth Forestry Institute, Oxford, are described briefly below and in more detail in Special Appendix 3. They are suggested as a basis from which some standard procedures could probably be derived.

\section{Procedure for measurement of ring width and structure}

Ring width can be measured easily by smoothing the axial surface of wood specimens, taken in the radial direction, and traversing them with a linear measuring microscope. The simplest forms of such microscopes are read from linear and vernier scales, a slow and tedious process, liable to lead to inaccuracies. Systems to record the output of linear measuring microscopes in digital form, with a paper tape output, are easily constructed, at a reasonable cost, and can be operated very quickly with great accuracy. (See Special Appendix 3 for further information.)

The dimensions of major structural divisions within rings, such as early wood and late wood, ring porous zones and the occurrence of reaction wood, can be recorded in the same way as for whole ring widths.

\section{Procedure for measurement of density}

Density is often the most important of the key index features, and is usually measured from the oven dry weight and green volume of specimens, or from the weight and volume at a fixed moisture content, e.g. $12 \%$. If information is required on the density of wood at various moisture contents, then the volumetric shrinkage with changes in moisture content must also be known. 
For many purposes, and where specimens of a suitable size and shape are available, values for density can be calculated accurately and quickly by the usual methods of gravimetric and volume measurement, e.g. from whole increment cores, or sections of cores, or from strength test specimens machined to a standard size. If, however, information is required on the variation in density from ring to ring or within annual rings, which may be of importance in some applications, including pulping, it is usually advisable to use one of the scanning methods that have been recently developed. An example of the sort of information than can be recorded by the system of X-radiography and densitometric scanning of radiographs, used at the Commonwealth Forestry Institute, Oxford, is given in Special Appendix 3 and in Hughes and Sardinha (1975) and Plumptre (1976).

To obtain accurate values for the mean density of whole trees is not easy, because it is difficult to measure the volumes of large pieces of wood of irregular shape (but see Carter, 1974). In fact, a sampling system of the type described above, designed to investigate within-tree patterns of variation, will give a very precise estimate of whole tree values, with less effort than is required for actual whole tree measurements and probably without much statistical bias. Since bias is also likely to be similar in all trees, this system should give very accurate comparisons of the whole tree mean values for the density of different trees, which is what is usually required.

\section{Procedure for measurement of grain angle}

The angle of deviation of the grain in trees is usually measured either on discs or on borings. It is essential, for the measurement of grain angle, to mark a datum on the disc or boring to show the alignment of the axis of the tree, or alternatively to record the actual deviation from the axis under the bark, preferably when the tree is standing. An instrument for the purpose can be constructed from a low power or a telescopic objective and a goniometer eyepiece.

In the laboratory, changes in the grain angle of wood specimens (borings or discs) from the pith outwards, can be easily recorded with a simple low-power microscope, fitted with an angled tube and a goniometer eyepiece can be geared to a digitiser, so that the angles measured can be recorded directly on punch tape by data logger systems of the sort described in Special Appendix 3.

\section{Procedure for measurement of shrinkage and movement}

In normal wood, the shrinkage that occurs in drying from the green state to about $12 \%$ moisture content is small, but radial shrinkage of up to about $8 \%$, tangential shrinkage of up to about $13 \%$ and total volumetric shrinkage of the order of $20 \%$ is not uncommon. Shrinkage or swelling of wood in use (movement), caused by alteration of its equilibrium moisture content and corresponding to variation in the relative humidity of the atmosphere, may be of the order of up to $4.5 \%$ for the combined tangential and radial movement. For the purpose of comparison, movement is often calculated for a fixed range of relative humidities, e.g. from $60 \%$ to $90 \% \mathrm{RH}$ at $25^{\circ} \mathrm{C}$. Because shrinkage or swelling is determined in part by the amount of cell wall material, the change of dimension in either the 
radial or tangential plane may vary considerably in different parts of the same tree, e.g. from pith to bark on the same radius. The detailed measurement of within-tree patterns of shrinkage and swelling is, therefore, likely to be a laborious process, and is rarely attempted. A mean value for radial and for tangential shrinkage will give some indication of likely behaviour in drying, particularly if the overall range of shrinkage values and some mean tree values are also known.

Values for shrinkage of this sort are usually calculated from caliper measurements of the $2 \mathrm{~cm} \times 2 \mathrm{~cm} \times 6 \mathrm{~cm}$ standard compression strength test pieces or from pieces of a similar size. If specimens are taken in the manner recommended for the general utilization tests, a good estimate will be obtained of mean values and the range of values for shrinkage.

This information may be adequate for many purposes, but it seems likely that much greater use could be made of shrinkage as a key index feature, if we had adequate techniques for measurement of patterns of variation in shrinkage and for determining the relationships between shrinkage and other use characteristics of wood.

Further suggestions for the measurement of shrinkage are discussed in the next section under Special Tests.

\section{Procedure for measurement of cell dimensions}

With species that are likely to be used for paper making, measurement of the lengths and cross-sectional dimensions of cells may be required (usually only the tracheids or fibres are measured).

To obtain cell length, wood specimens are macerated, and the images of tracheids or fibres separated in this way are then projected, at a suitable magnification, on a translucent screen or on a papered surface. Measurement is commonly done with map measurers reading in centimetres. These can be modified to give an electrical pulse for every $1 \mathrm{~cm}$ or $.5 \mathrm{~cm}$ of traverse of the image, so that with a suitable interface the output can be recorded through a data logger system on punch tape.

Cell cross-sectional dimensions can be measured on macerated material but there are problems with this method in distinguishing between radial and tangential dimensions, and it is rather slow. An alternative method is to take measurements on transverse surfaces of wood specimens that have been polished on a belt sander, using a belt with a fine grade (no. 400) of silicon carbide. A few minutes only are required to produce a smooth and evenly polished surface, with no apparent distortion, that is suitable for examination by an incident light microscope and measurement by a screw micrometer eyepiece, at magnification of $\times 300$ to $\times 500$ (Hughes and Lantican, 1973). Screw micrometer eyepieces suitable for such measurements can be fitted with optical digitisers and interfaced to data logging systems.

\section{(iii) Analysis and presentation of data and results}

Here again it is neither possible nor advisable to attempt to prescribe a standard method for the presentation and analysis of data and 
results from the various tests that may be required to ascertain the effects of genetic factors and environmental conditions on wood quality. Some techniques that are likely to be widely applicable are described briefly here and are explained in greater detail in the references cited.

\section{Within-tree patterns of variation}

If the within-tree sampling system put forward above and in Special Appendix 1 is adopted, a useful description of within-tree variation can be obtained by regression analyses. These will show the way in which a particular property, e.g. density, changes with age, with distance from the pith, or with height above ground (see e.g. Burley, 1970; Burley and Andrew, 1970; Andrew and Burley, 1972; Burley, Hughes, Franklin et a1., 1973; Burley, Hughes, Templeman et al., 1973; Plumptre, 1976.

This approach is of value to establish the general trends of withintree variation of wood characteristics for a species or provenance, to provide a standard against which:-

(a) trends associated with particular origins or locations can be examined to make comparisons of the overall effects of, $\underline{e} . \mathrm{g}$. different combinations of site factors or of different silvicultural treatments,

(b) patterns of variation of individual trees (e.g. candidate "plus trees" in selection programmes) can be examined, in relation to the general trend, to identify those that have the most desirable features, such as a small juvenile core, a great degree of uniformity or high mean values for wood properties.

As an aid to the comparison of the pattern of variation in different trees, computer programs have been developed at the Commonwealth Forestry Institute, Oxford, with which information on the variation in wood properties from pith to bark is presented graphically, on a reduced scale, so that all the height levels sampled can be shown together (see example in Special Appendix 3). Recently, this technique has been further developed to present profile or contour diagrams of the whole within-tree patterns of variation of wood characteristics from which trees can be compared easily by visual inspection.

\section{Whole tree mean values}

It should be recognized that the average value of all the samples taken from a tree may differ considerably from the mean whole tree value. Consequently, if whole tree values are required, an appropriate withintree sampling system must be used that can be correctly weighted in subsequent calculations. A valid system of weighting (such as by proportion of total volume or cross-sectional area must be also used to obtain a good estimate.

\section{Mean values per hectare}

If the system adopted for selecting the trees to be measured provides a representative sample of adequate size of the population, then the calculation of mean values on land area basis is a straightforward 
matter. It should be recognized, however, that estimates of mean whole tree values, if calculated from a series of within-tree samples, may be biassed although very precise. Consequently, when comparing different sites or different treatments at the same site, it is advisable to use the same sampling method throughout. When calculating mean values per hectare from sample tree values, an appropriate system of weighting must be used to take account of the difference in size and volume of sample trees and of the size and volume distribution of trees in that particular crop.

\subsection{Specialized tests}

Situations will arise where investigations of wood properties are required of a sort not covered in the standard pilot and general utilization tests already described. There is little point in attempting to anticipate and to list all such circumstances, and a few cases only are given here, by way of illustration, where it seems likely that special studies may be required.

For example, some species of low average density may have strength properties that are marginal for structural purposes. It may then be advisable to investigate the specific strength (that is the strength/ weight ratio) of wood in different parts of the bole, and also the mean tree values of this factor for different trees from the same site. If there is considerable within-tree and between-tree variation in specific strength, it may be possible to increase the overall average strength by appropriate silvicultural treatment or by selection and breeding. This is not a fanciful notion - there is already evidence of considerable between-tree and within-tree variation in the specific strength of some species.

Another situation which would seem to warrant special investigation is the marked degrade in drying shown by some species, e.g. some eucalypts, particularly when fast grown - a very serious and limiting factor in their use. An investigation of the patterns of occurrence of growth stresses and of shrinkage, and an attempt to identify associated growth factors, would be a formidable task - but an undertaking that might repay the effort and expense.

It is suggested that, if problems arise which seem to require special studies, these should be referred to IUFRO (International Union of Forestry Research Organizations) and brought to the attention of the appropriate research organizations thoughout the world. In this way it should be possible to form alliances of research workers with similar interests, who are prepared to exchange ideas, information, techniques and materials.

\section{FOOTNOTE:}

Owing to the size of the appendices to this section they are not included in the Manual. They are, however, available, on request, from The Commonwealth Forestry Institute, South Parks Road, Oxford, England.

The Special Appendices concerned are:-

Special Appendix 1. Standard methods for recording information when collecting samples for wood quality tests.

Special Appendix 2. Laboratory procedures for generaly utilization tests.

Special Appendix 3. The wood structure index approach used at the Commonwealth Forestry Institute, Oxford, for the evaluation of wood quality. 
Andrew, I.A. and Burley, J. (1972). Variation of wood quality of Pinus merkusii Jungh. and de Vriese; five trees of Burma provenance grown in Zambia. Rhod. J. agric. Res. 10, 183-202.

Andrew, I.A. and Burley, J. (1973). Data processing for international provenance research. In Tropical provenance and progeny research and international cooperation, eds. J. Burley and D.G. Nikles. Commonw. For. Inst., Oxford. Pp. 353-6.

Ashton, P.S. (1976). An approach to the study of breeding systems, population structure and taxonomy of tropical trees. In Tropical trees: variation, breeding and conservation, eds. J. Burley and B.T. Styles. Academic Press, London. Pp. 35-42.

Astle, W.L., Webster, R. and Lawrance, C.J. (1969). Land classification for management planning in the Luangwa Valley of Zambia. J. appl. Eco1. 6, 143-69.

Barber, H.N. (1955). Gene substitutions in eucalypts. Evolution 9, $1-14$.

Barber, H.N. (1965). Selection in natural populations. Heredity 20, 551-72.

Barner, H. (1971). Procurement of Douglas fir seed for provenance research. Pap. 15th IUFRO congress, Gainesville. Pp. 9.

Barner, H. (1974a). Classification of sources for procurement of forest reproductive material. In Report on the FAO/DANIDA training course on forest tree improvement, Kenya, 1973. FAO/DEN/TF112, Rome. Pp. 110-38.

Barner, H. (1974b). Exploration, collection and evaluation of forest reproductive material. In Report on the FAO/DANIDA training course on forest tree improvement, Kenya, 1973. FA0/DEN/TF112, Rome. Pp. 139-50.

Barrett, R.L. and Mullin, L. (1968). A review of introductions of forest trees in Rhodesia. Rhod. Bu1l. For. Res. No. 1. Pp. 227.

Bawa, K.S. (1974). Breeding systems of tree species of a lowland tropical community. Evolution 28, 85-92.

Bawden, M.G. (1965). A reconnaisance of the land resources of eastern Bechuanaland. J. appl. Ecol. 2, 357-65.

Bawden, M.G. and Carroll, D.M. (1968). The land resources of Lesotho. Land Resources Division, Directorate of Overseas Surveys, Land Resources Study, No. 3. Pp. 89. 
Bawden, M.G., Carroll, D.M. and Tuley, P. (1972). The land resources of North East Nigeria. Vol. 3. The land systems. Land Resources Division, Overseas Development Administration. Land Resources Study, No. 9. Pp. 466.

Bennett, E. (1970). Tactics of plant exploration. In Genetic resources in plants - their exploration and conservation, eds. 0.H. Frankel and E. Bennett. IBP Handbook No. 11, Blackwe11, Oxford. Pp. 157-79.

Bouvarel, P. (1970). The conservation of gene resources of forest trees. In Genetic resources in plants - their exploration and conservation, eds. 0.H. Frankel and E. Bennett. IBP Handbook No. 11, Blackwe11, Oxford. Pp. 523-9.

Bradshaw, A.D. (1952). Populations of Agrostis tenuis resistant to lead and zinc poisoning. Nature $169,1098$.

B.S.I. (1957). Methods of testing small clear specimens of timber. British Standard No. 373. Pp. 35 .

Burley, J. (1970). Variation in wood properties of Pinus kesiya Royle ex Gordon (syn. $\underline{P}$. khasya Royle; $\underline{\mathrm{P}}$. insularis Endlicher) : eighteen trees of Burma provenance grown in Zambia. Wood Sci. and Technol. $41,255-66$.

Burley, J. (1973). Generalised analysis of provenance experiments. In Tropical provenance and progeny research and international cooperation, eds. J. Burley and D.G. Nikles. Commonw. For. Inst., Oxford. Pp. 388-91.

Burley, J. and Andrew, I.A. (1970). Variation in wood properties of Pinus kesiya Royle ex Gordon (syn. $\underline{P}$. khasya Royle; $\underline{P}$. insularis Endlicher) : six trees of Assam provenance grown in Zambia. Wood Sci. and Technol. 4, 195-212.

Burley, J. and Turnbul1, J.W. (1970). Proposed standard procedures for the FAO/FRI/CFI international provenance trial of Pinus kesiya Royle ex Gordon (syn. $\underline{P}$. insularis Endlicher; $\underline{P}$. khasya $\overline{\text { Royle) }}$. Mimeographed, Commonw. For. Inst., Oxford. $\mathrm{Pp}-25$.

Burley, J., Hughes, J.F., Templeman, H.J. and Gourlay, I.D. (1973). Summary report on variation in density of Pinus occidentalis from Cuba. In Selection and breeding to improve some tropical conifers, eds. J. Burley and D.G. Nikles. Commonw. For. Inst., Oxford. Pp. 552-60.

Burley, J., Hughes, J.F., Franklin, P. and Gourlay, I.D. (1973). Summary report on variation in density and tracheid length of ten trees of Pinus caribaea var. hondurensis Barr. and Golf. from Jamaica. In Selection and breeding to improve some tropical conifers, eds. J. Burley and D.G. Nikles. Commonw. For. Inst., Oxford and Dept. For., Queensland. Vol. 2. Pp. 72-85. 
Burley, J. and Lever, K.G. (1973). The application of biochemical methods in tropical provenance research. In Tropical provenance and progeny research and international cooperation, eds. J. Burley and D.G. Nikles. Commonw. For. Inst., Oxford. Pp. 336-43.

Burley, J., Andrew, I.A. and Palmer, J.R. (1974). Information collection, storage and retrieval in forestry. Pap. 10th Commonw. For. Conf., 0xford. Pp. 39.

Burley, J., Andrew, I.A. and Templeman, H. (1973). INTFORPROV - computer based data banks for international tropical provenance experiments. In Tropical provenance and progeny research and international cooperation, eds. J. Burley and D.G. Nikles. Commonw. For. Inst., 0xford. Pp. 357-65.

Callaham, R.Z. (1964). Provenance research: investigation of genetic diversity associated with geography. Unasylva 18, 40-50.

Carter, D.T. (1974). The determination of saturated volumes of large timber specimens in basic density work using a standard single pan balance of small maximum capacity. Commonw. For. Rev. 53, 16-18.

Christian, C.S. and Stewart, G.A. (1952). General report on the survey of the Katherine-Darwin Region, 1946. Commonwealth Scientific and Industrial Research Organisation, Melbourne. Land Research Series, No. 1. Pp. 156 .

Clausen, J., Keck, D.D. and Hiesey, W.M. (1940). Experimental studies on the nature of species. I. The effects of varied environments on western N. American plants. Publs. Carnegie Instn. 520. Pp. 452.

Cochran, W.G. (1953). Sampling techniques. Wiley, New York. Pp. 330 .

Cochran, W.G. and Cox, G.M. (1957). Experimental designs. (2nd. ed.) Wiley, New York. Pp. 611

Coile, T.S. (1952). Soil and the growth of forests. Advances in Agronomy 4, 329-98.

Conkle, M.T. (1963). The determination of experimental plot size and shape in loblolly and slash pine. Tech. Rept. 17, School of Forestry, N.C. State College, Raleigh, U.S.A. Pp. 51.

Cox, D.R. (1958). Planning of experiments. Wiley, New York. Pp. 308.

Cox, G.M., Eckhardt, R.C. and Cochran, W.G. (1940). The analysis of lattice and triple lattice experiments in corn varietal tests. Research Bulletin 281, Iowa State College of Agriculture. Pp. 66.

Creech, J.L. (1970). Tactics of exploration and collection. In Genetic resources in plants - their exploration and conservation, eds. 0.H. Frankel and E. Bennett. IBP Handbook No. 11, Blackwe11, Oxford. Pp. 221-9. 
Dansereau, P. (1951). Description and recording of vegetation on a structural basis. Ecology 32, 172-229.

Dawkins, H.C. (1968). Statforms. Formats for elementary statistical calculation. Commonw. For. Inst., Oxford, Inst. Pap. No. 41. Pp. 26.

Dawkins, H.C. (1975). Statforms: 'Pro-formas' for the guidance of statistical calculations. Edward Arnold, London, U.K. Pp. 37.

Darwin, C. (1859). On the origin of species by means of natural selection. John Murray, London. Pp. 415.

Duncan, D.B. (1955). Multiple range and multiple F tests. Biometrics $11,1-42$.

F.A.0. (1968). A guide to tree species trials in tropical America. Rept. WS $/ 72017$, For. and For. Indust. Div., FAO, Rome. Pp. 80 .

F.A.0. (1969). Report of the first session of the FAO panel of experts on forest gene resources, FAO. Rome. Pp. 44.

F.A.0. (1972). Report of the second session of the FAO panel of experts on forest gene resources, FAO. Rome. Pp. 68.

F.A.0. (1974). Report of the third session of the FAO panel of experts on forest gene resources, FAO. Rome. Pp. 90.

F.A.0. (1975). Forest tree seed directory, FAO. Rome. Pp. 283.

Fisher, R.A. and Yates, F. (1963). Statistical tables for biological, agricultural and medical research ( 6 th ed.). Oliver and Boyd, Edinburgh and London. Pp. 146.

Fletcher, A.M., Howell, R.S. and Faulkner, R. (1968). Problems associated with the layout of progeny tests in Great Britain, with special reference to a recent plot size experiment. For. Comm. Stats. Sect. Pap. No. 143. Pp. 8. (Mimeo).

Fosberg, F.R. (1958). On the possibility of a rational general classification of humid tropical vegetation. In Proc. UNESCO Symp. on Humid Tropics Vegetation, Indonesia. Pp. 34-59.

Franklin, E.C. (1971). Statistical validity of single tree plots in forest genetics research. Silvae Genet. 20, 73-5.

Freeman, G.H. and Perkins, J.M. (1971). Environmental and genotypeenvironmental components of variability. VIII. Relations between genotypes grown in different environments and measures of these environments. Heredity 27, 15-23.

Freese, F. (1962). Elementary forest sampling. U.S.D.A. Agric. Handbk. No. 232. Pp. 91. 
Gilmour, J.S.L. and Gregor, J.W. (1939). Demes: a suggested new terminology. Nature 144, 333-4.

Granhof, J.J. (1973). The "blotting paper method" - a standard nursery technique for provenance trials? In Tropical provenance and progeny research and international cooperation, eds. J. Burley and D.G. Nikles. Commonw. For. Inst., Oxford. Pp. 374-7.

Green, J.W. (1968). Tables of random permutations. For. Timber Bur., Canberra, Australia. Bul1. No. 44. Pp. 161.

Gregor, J.W. (1939). Experimental taxonomy IV. New Phytol. 38, 293-322.

Hans, A.S. (1972). Development of an instrument for assessment of stem straightness. Commonw. For. Rev. $51,336-45$.

Hanse11, J.R.F. and Wall, J.R.D. (1974). Land resources of the British Solomon Islands Protectorate. Vol. 2. Guadalcanal and the Florida Islands. Land Resources Division, Ministry of Overseas Development. Land Resources Study No. 18. Pp. 221

Holdridge, E.R. (1967). Life zone ecology. Rev. ed. Tropical Science Centre, Costa Rica. Pp. 206.

Holmes, G.D. and Buszewicz, G. (1958). The storage of seed of temperate forest tree species. For. Abstr. 19, 313-22; 455-76.

Hughes, J.F. and Sardinha, R.M.A. (1975). The application of optical densitometry in the study of wood structure and properties. J. Microscopy 104, 91-103.

Huxley, J.S. (1939). Clines: an auxiliary taxonomic principle. Nature $142,219-20$.

International Code of Botanical Nomenclature. (1972). International Association of Plant Taxonomy. Oosthoek's Uitgeversmaatschappij N.V., Utrecht, Netherlands. Pp. 426.

Jackson, D.S. (1962). Parameters of site for certain growth components of slash pine (Pinus elliottii Engelm.). Sch. For., Duke Univ. Bul1. No. 16. Pp. 118 .

Jeffrey, C. (1973). Biological nomenclature. Edward Arnold, London. Pp. 69.

Jenkin, R.N. (1973). Forest soil surveying with special reference to the selection of sites for forest plantations. Fed. Dept. For. Res., Nigeria, Technical Note No. 26. Pp. 36.

Jones, J.R. (1969). Review and comparison of site evaluation methods. U.S.D.A. For. Serv. Res. Pap. No. RM-51. Pp. 27. 
Jones, N. and Burley, J. (1973). Seed certification, provenance nomenclature and genetic history in forestry. Silvae Genet. 22, 53-8.

Jones, N. and Burley, J. (1975). Notes on tropical and semi-tropical species other than eucalyptus species and teak. In Seed orchards, ed. R. Faulkner. For. Comm. Bul1. 54, 131-4.

Jong, K. and Lethbridge, A. (1967). Cytological studies on the Dipterocarpaceae. I. Chromosome numbers of certain Malaysian genera. Notes R. bot. Gdn. Edinb. 27, 175-84.

Kemp, R.H. et al. (1972). International co-operation in the exploration, conservation and development of tropical and subtropical forest gene resources. Pap. 7 th World For. Congr., Argentina. Pp. 15.

Knight, R.A.G. (1956). Revised manual for conducting trials of timbers for plywood manufacture. FPRL, Princes Risborough, Misc. Pap. Pp. 14.

Küchler, A.W. (1949). A physiognomic classification of vegetation. Ann. Ass. Am. Geogr. 39, 201-10.

Langlet, 0. (1934). Om variationen hos tallen Pinus sylvestris och dess

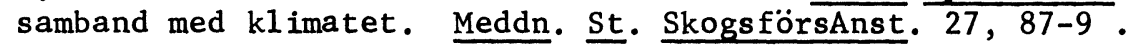

Lantican, C.B. and Hughes, J.F. (1973). A rapid method for specimen preparation and for measurement of cell cross-sectional dimensions. IAWA Bu11. 4, 11-18.

Larsen, E. (1969). Seed procurement for research. In Proc. 2nd World Consult. For. Tree Breed., Vo1. 2 (Paper: 949-578/3), FAO, Rome.

Lavers, G.M. (1969). The strength properties of timbers (Metric edition). FPRL, Princes Risborough, For. Prod. Bul1. No. 50 (rev.). Pp. 62.

Ledig, F.T. (1974). An analysis of methods for the selection of trees from wild stands. For. Sci. 20, 2-16.

Leenhouts, P.W. (1968). A guide to the practice of herbarium taxonomy. Reg. Veg. 58,60 .

Leuchars, D. (1965). The planning and practice of trials of exotic species. E. Afr. agric. For. J. 31, 83-90.

Lever, K.G. and Burley, J. (1974). The application of biochemical methods in forestry. Pap. 10th Commonw. For. Conf., Oxford. Pp. 20.

Lines, R. (1965). Provenance and the supply of forest tree seed. Q. J1 For. $59,7-15$. 
Lines, R. (1967). Standardization of methods for provenance research and testing. Report of the IUFRO Section 22 Working Group Meeting at Pont-a-Mousson, September 6th-9th, 1965. In Proc. 14th IUFRO Congr., Munich. Pp. 672-714.

Magini, E. (1962). Forest seed handling, equipment and procedures. II - Seed treatments, storage, testing and transport. Unasylva 16, 20-35.

Matthews, J.D. (1964). Seed production and certification. Unasylva 18, 104-18.

Mayr, E.M. (1963). Animal species and evolution. Harvard Univ. Press, Cambridge, Mass. Pp. 797.

McCulloch, J.S.G. (1965). Tables for the rapid computation of the Penman estimate of evaporation. E. Afr. agric. For. J. 30, 286-95.

Mehra, P.N. (1972). Cytogenetical evolution of hardwoods. Nucleus 15, 64-83.

Mergen, F. (1963). Ecotypic variation in Pinus strobus. Ecology 44, 716-27.

Mergen, F., Burley, J. and Furnival, G.M. (1974). Provenance-temperature interactions in four coniferous species. Silvae Genet. 23, 200-10.

Morandini, R. (1961). Forest seed handling equipment and procedures. I - Seed production, collection and extraction. Unasylva 15, 185-99.

Moreau, R.E. (1938). Climatic classification from the standpoint of East African biology. J. Ecol. 26, 467-96.

Murdoch, G., Webster, R. and Lawrance, C.J. (1971). Atlas of land systems in Swaziland. Soil Sci. Lab., Dept. Agric. Sci., Univ. Oxford. $\mathrm{Pp}$. 49 .

Nanson, A. (1970). Héritabilité et le gain d'origine génétique dans quelques types d'expériences. Silvae Genet. 19, 113-21.

Nelder, J.A. (1962). New kinds of systematic designs for spacing experiments. Biometrics 18, 283-307.

O.E.C.D. (1974). OECD scheme for the control of forest reproductive material moving in international trade. $\overline{\mathrm{OE}} \overline{\mathrm{D}, \mathrm{Par}} \overline{\mathrm{ts}}$.

011ier, C.D. et al. (1969). Terrain classification and data storage: land systems of Uganda (M.E.X.E. Rept. No. 959). Soil Sci. Lab., Dept. Agric., Univ. of Oxford. Pp. 234.

Pearce, S.C. (1965). Biological statistics : an introduction. McGrawHill, New York. Pp. 212. 
Pearson, E.S. and Hartley, H.0. (1966). Biometrika tables for statisticians. Volume 1. Cambridge University Press. Pp. 264.

Pearson, R.G. (1969). Sampling of timber for evaluation of species properties. CSIRO Div. For. Prod. Australia, For. Prod. Tech. Note No. 5. Pp. 31 .

Pearson, R.S. and Stevens, W.C. (1929). The measurement of distortion on converted timber. FPRL, Princes Risborough, Misc. Pap. No. 5. Pp. 11 .

Penman, H.L. (1963). Vegetation and hydrology. Tech. Commun., Bureau of Soils, Harpenden, 53. Pp. 124.

Pereira, H.C. and Hosegood, P.H. (1962). Comparative water-use of softwood plantations and bamboo forest. J. Soil Sci. 13, 299-313.

Pereira, H.C., Dagg, M. and Hosegood, P.H. (1962). The water balance of the cultivated and control catchments. E. Afr. agric. For. J. (special issue) $27,118-22$.

Plumptre, R.A. (1976). Some techniques used in the study of wood density. Pap. 16th IUFRO Congr., 0slo. Pp. 6.

Pryor, L.D. (1963). Provenance in tree improvement with particular reference to Eucalyptus. In Proc. World Consultn. For. Genet. Tree Impr., Vol. I (Paper 3/2). Pp. 6. FAO. Rome.

Ra1ston, C.W. (1964). Evaluation of forest site productivity. Internat. Rev. For. Res. 1, 171-201.

Redding, L.W. (1958). The resistance of various timbers to impregnation. FPRL, Princes Risborough, Misc. Pap. Pp. 46.

Rennie, P.J. (1963). Methods of assessing site capacity. Commonw. For. Rev. 42, 306-17.

Rijks, D.A., Owen, W.G. and Hanna, L.W. (1970). Potential evaporation in Uganda. Government Printer, Entebbe.

Rohlf, F.J. and Sokal, R.R. (1969). Statistical tables. W.H. Freeman and Co., San Francisco. Pp. 253.

Scott, R.M., Webster, R. and Lawrance, C.J. (1970). Atlas of the land systems of western Kenya (M.E.X.E. Rept. No. 1112). Soil Sci. Lab., Dept. Agric., Univ. Oxford. Pp. 363.

Seal, D.T. et al. (1965). Collection of cones from standing trees. Rev. ed. For. Comm. For. Rec. No. 39. H.M.S.0., London. Pp. 39.

Sea1, H.L. (1964). Multivariate statistical analysis for biologists. Methuen, London. Pp. 209. 
Snedecor, G.W. and Cochran, W.G. (1967). Statistical methods. Iowa State Univ. Press. Pp. 593.

Soest, J. van, Ayral, P., Schober, R. and Hummel, F.C. (1959). Recommendations on the standardization of symbols in forest mensuration. Internat. Union For. Res. Organ., London. Pp. 32.

Styles, B.T. and Vosa, C.G. (1971). Chromosome numbers in the Meliaceae. Taxon 20, 485-99.

Theron, K. (1973). Report on initial climatic classification of East Africa. Unpublished Report, Commonwealth Forestry Institute, Oxford.

Thornthwaite, C.W. and Mather, J.R. (1957). Instructions and tables for computing potential evapotranspiration and the water balance. C1imatology 10, 185-311.

Toleman, R.D.L. and Pyatt, D.G. (1974). Site classification as an aid to silviculture in the Forestry Commission of Great Britain. Pap. 10th Commonw. For. Conf. 20 pp.

Turesson, G. (1922). The species and variety as ecological units. Hereditas $3,100-13 ; 211-350$.

Turnbul1, J.W. (1975). Assessment of seed crops and the timing of seed collections. In Report on the FAO/DANIDA training course on forest seed collection and handling, Vol. 2. FOR:TF-RAS 11 (DEN), FAO Rome. Pp. 79-94.

U.S. Dept. Agric. (1951). Soil survey manual. U.S.D.A. Handbk. No. 18. Pp. 503.

Valentine, D.H. and Löve, A. (1958). Taxonomic and biosystematic categories. Brittonia 10, 153-66.

Vyskot, M. (1963). Standardisation of methods in provenance research and testing. Pap. FAO World Consultn. For. Genet. Tree Impr., Stockholm. No. FAO/FORGEN 63/-3/5. Pp. 7.

Wa11, J.R.D. and Hansell, J.R.F. (1974). Land resources of the British Solomon Islands Protectorate. Vol. 3. Malaita and Ulawa. Land Resources Division, Ministry of Overseas Development. Land Resources Study No. 18. Pp. 125 .

Wang, B.S.P. (1973). Collection, processing and storing tree seed for research use. In Proc. IUFRO Int. Symp. Seed Processing, Bergen, Norway, 1973. Royal Coll. For., Stockholm, Sweden. Vol. 1, Pap. 17. Pp. 12.

Wang, B.S.P. (1974). Tree seed storage. Publ. Canad. For. Serv. No. 1335. $32 \mathrm{pp}$.

Woodhead, T. (1968). Studies of potential evaporation in Tanzania. E. Afr. Agric. For. Res. Org., Nairobi. Pp. 60 . 
Wright, J.W. (1962). Genetics of forest tree improvement. F.A.O. For. and For. Prod. Stud. No. 16, Rome. Pp. 399.

Yates, F. (1939). The recovery of inter-block information in variety trials arranged in three dimensional lattices. Ann. Eugen. 9, 136-56. 
Appendix 1. Glossary and symbols.

Appendix 1.1. Glossary of some terms used in the Manual (Wherever possible standard IUFRO, FAO or SAF definitions are given)

Aneuploid. An individual or cell having one, two or a few whole chromosomes more or less than the basic number of the species in question.

Biometry (Biometrics). The application of statistics to biological problems.

Biosystematics (Biosystematy). Taxonomic studies involving morphology, cytogenetics, ecology and phytogeography.

Block. A set of items or experimental units under treatment or observation, which have been grouped to minimize environmental effects or initial differences between items or units in respect of the variables being studied, $\underline{e} . \underline{g}$. a set of contiguous experimental plots. It is not necessary for all treatments to appear in one block but if they do Block is synonymous with Replication; specially arranged subsets form Incomplete Blocks.

Design. A method of arranging sample or experimental units to minimize the effects of uncontrolled variation caused by natural factors, e.g. soil fertility, and to make it possible to estimate the magnitude of such effects in relation to those due to variations in treatment.

Exotic. Commonly used to refer to a plant introduced from a foreign country. However, strictly it should refer to a plant grown anywhere outside its natural range (synonymous with Non-native)

Flower. Strictly an angiospermous reproductive structure bearing pistils, stamens or both, and usually sepals and petals. The socalled flower of conifers is the male or female Strobilus before and during pollination.

Genetics. Genetics is the basic science dealing with causes of resemblances and differences among organisms related by descent. It takes into account the effects of genes and the environment. When the basic knowledge of genetics is applied to breeding trees, the effort is preferably referred to as forest tree breeding or forest tree improvement.

Genotype. (1) An individual's hereditary constitution, with or without phenotypic expression of the one or more characters it underlies. Also the gene classification of this constitution expressed in a formula. The genotype is determined chiefly from performance of progeny and other relatives. It interacts with the environment to produce the phenotype. (2) Individual(s) characterized by a certain genic constitution.

Genotype-environment interaction. The failure of entries to maintain the same relative ranks and level of differences when tested in different environments. The tests are planted at more than one location or under more than one cultural condition. 
Heritability. Degree to which a character is influenced by heredity as compared to environment. Narrow-sense heritability is the fraction of total variation that is contributed by additive effects of genes, i.e. it is the ratio of additive genotype variance to phenotypic variance. Broad-sense heritability, applicable to vegetatively propagated species, includes also non-additive effects. High heritability indicates that individual phenotypes are indicative of their genotypes. If calculated from parent-progeny data it estimates the degree of resemblance between parent and progeny.

Indigenous. Native to a specified area; not introduced.

Non-native. See Exotic.

Origin. For an indigenous stand of trees the origin is the place in which the trees are growing; for a non-indigenous stand the origin is the place from which the seed or plants were originally introduced.

Phenotype. An organisms as observed, $\underline{i}$.e. as judged by its visually perceptible characters resulting from the interaction of its Genotype with the environment. Similar phenotypes do not necessarily breed alike.

Plot. Usually the smallest experimental unit in a replicated comparative experiment, $\mathrm{e} \cdot \mathrm{g}$. One replication of one provenance in a trial of many provenances with several replications.

Polyploid. An individual or a cell having three (triploid), four (tetraploid), five (pentaploid) or more complete sets of chromosomes instead of two as in diploids.

Population. Genetically, a group of similar individuals related by descent and so delimited in range by environment or endogenous factors as to be considered a unit. In cross-bred organisms the population is often defined as the interbreeding group.

Progeny test. Evaluation of parents by comparing the performance of their offspring. Accuracy is usually gained because several to many offspring per parent are evaluated under more controlled conditions than exist for the parent.

Provenance. The place in which any stand of trees is growing. The stand may be indigenous or non-indigenous.

Replication. Verb: Applying a treatment or set of treatments more than once to increase the precision of comparisons and to provide an assessment of the variability among experimental units treated alike.

Noun: One complete set of all experimental units in a comparative experiment (see also Block and Design).

Sample. A part of a Population, consisting of one or more sampling units selected and examined as representative of the whole.

Seed Source. See Provenance and Origin. 
Selection. Often synonymous with artificial selection, which is the choice by the breeder of individuals for propagation from a larger population. Artificial selection may be for one or more desired characteristics. It may be based on the tree itself (phenotypic), or on the tree's progeny or other relatives (genotypic). Refers also to the tree selected.

Species. One or more populations, the individuals of which can interbreed, but which in nature cannot exchange genes with members belonging to other species. The main category of taxonomic classification.

Stand. A community of trees possessing sufficient uniformity of composition, constitution, age, spatial arrangement or condition, to be distinguishable from adjacent communities, so forming a silvicultural or management entity.

Strobilus. See Flower.

Taxonomy. Classification of organisms, including identification and nomenclature, according to a natural (chiefly morphological) system that seeks to depict evolvement.

\section{The terminology of policy and planning}

Confusion often arises because the terms "objectives", "policy", "strategy", etc. tend to be used to mean different things in different places - not infrequently in different places in a single document. As far as possible in the present study the terms are used to mean the following:-

Objectives are formulated by governments and therefore based mainly upon political considerations.

Goals and targets interpret the political objectives in physical and quantitative terms.

Policies or strategies indicate how the objectives, and the consequent goals and targets, are to be achieved and are formulated by the executive agency, $\underline{e} . \underline{\text {. }}$. the forest service.

Policy instruments and measures are the tools, such as legislation and taxes, employed to pursue the policies.

Programmes, projects and activities are the concrete elements which translate the plans for achieving the objectives into actual development.

The traditional government "forest policy" is therefore usually a statement of "objectives" as defined here. 


\section{Appendix 1.2. A selection of symbols for forest mensuration}

Note. Lower case symbols refer to individual tree values; upper case symbols refer to crop values per unit area.

\section{Principal symbols}

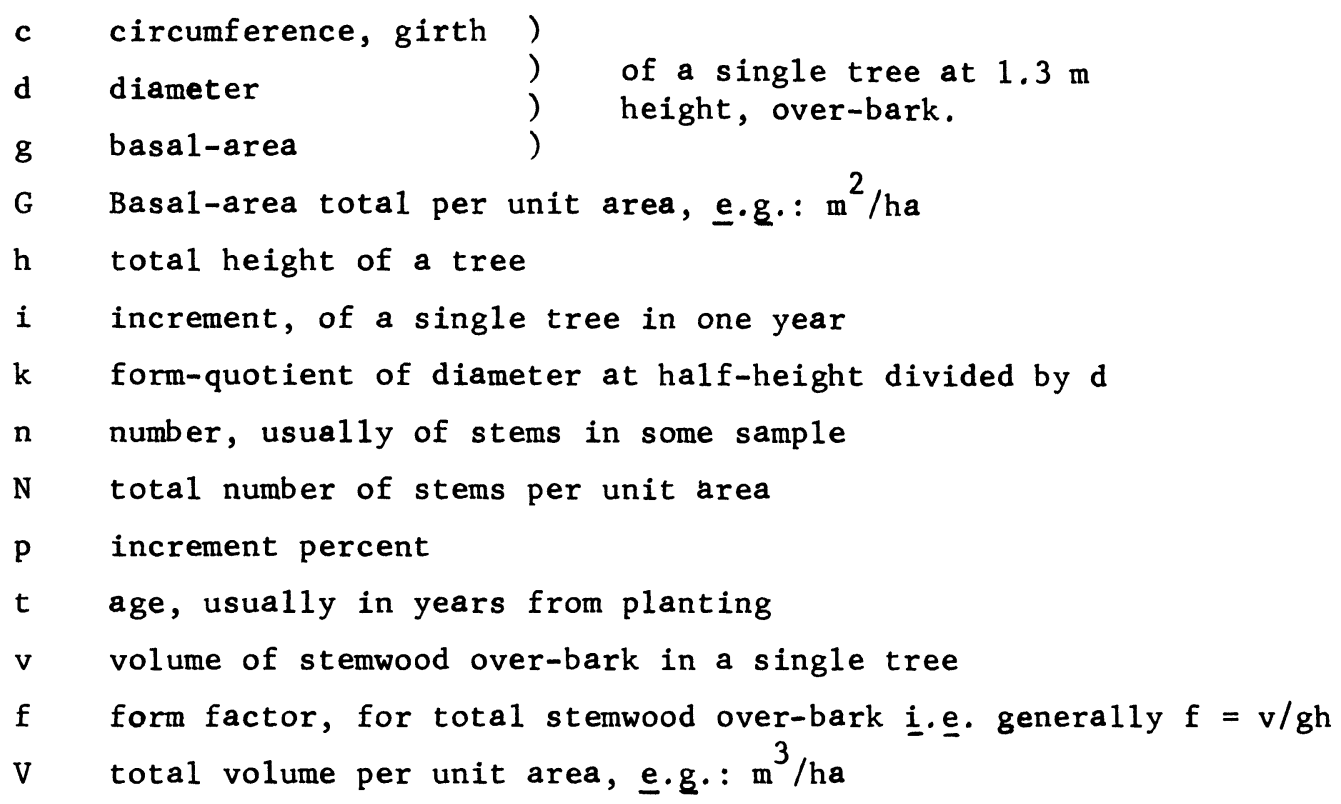

A11 the above may be qualified by subscripts or other modifications, of which the more useful recommendations are:-

\section{Qualified symbols}

$\overline{\mathrm{d}}$ arithmetic mean diameter of a stand, $\underline{1} . \underline{\mathrm{e}}$. (d) $/ \mathrm{n}$

$\mathrm{dg}$ diameter of the tree of mean basal-area

$\bar{g}$ basal-area of the tree of mean basal-area in a stand, $\underline{i} \cdot \underline{e} \cdot \mathrm{G} / \mathrm{n}$

$\bar{h}$ arithmetic mean height of a stand, $\underline{i} \cdot \underline{e}$. $(h) / n$

hg height of the tree of mean basal-area

$h \bar{d}$ height of the tree of arithmetic mean diameter

$h_{\text {dom }}$ "top-height", mean height of the one hundred thickest trees per hectare $i_{d}$ increment in diameter of a single tree in one year

$v_{7}$ volume of stem-wood (of a single tree) exceeding $7 \mathrm{~cm}$ diameter over-bark $\underline{i} \cdot \underline{\text { e. }}$ volume to a $7 \mathrm{~cm}$ diameter top

$v_{b}$ total above-ground volume of a tree, all branchwood included 


\section{Appendix 2. Seed collection}

Appendix 2.1. Annotated list of equipment which may be needed for collection of seed, site information and herbarium specimens

\section{A. Seed collection}

Seed containers (field). Sacks and bags (can be re-used).

Seed containers (despatch). Cotton bags, canvas sacks (despatched with seed).

Tree markers, e..g. plastic tape.

Climbing equipment. Foot spurs or tree bicycles or ladders. Safety belt, safety ropes, safety helmets, tool lines.

Seed cutters, e.g. cone hooks, cone rakes, pruning shears, secateurs (hand).

Plastic sheeting (heavy gauge) for protection when storing fruits, extracting seed, etc.

Binoculars for studying tree crowns, fruit development, etc.

Walkie-talkie radios (special permission may be needed).

Insecticidal and fungicidal powders for seed protection (use with care). Axe, saw, machete, knife.

Rope, string, labels, felt marking pens.

B. Site description

Notebook, description forms.

Maps (outline copies also for plotting).

Compass

Altimeter

Meteorological equipment (hygrometer, max/min thermometer).

Soil survey equipment (auger, colour charts, pH test kit).

Tree measuring equipment (altimeter, diameter tapes, bark gauge, etc.)

Camera and equipment (wide angle lens).

Tape recorder (battery powered).

Spade

\section{Specimen collection}

Botanical presses (may be made locally).

Drying papers (local newspapers will do).

Plastic bags

Specimen bottles

Preservative fluid

Increment borer (for wood samples).

Carpenter's brace and auger (for resin samples).

Insulated container (…g. ice box)

Hand lens

Insecticide spray (for herbarium material).

ALSO Medical supplies, camping equipment, vehicles and equipment as appropriate 
Appendix 2.2 Examples of seed collection data sheets

A. Commonwealth Forestry Institute, Oxford

Species: Pinus oocarpa Schiede

Country: Nicaragua

Seed No: K31

Store No: $1 / 71$

Department: Nueva Segovia

District: Dipilto

Latitude: $13^{\circ} 42^{\prime} \mathrm{N}$

Site: E1 Junquillo

Situation: At the western end and on the southern slopes of the Cordillera de Dipilto, which forms the northern boundary of Nicaragua at this point. The stands, which lie about $5 \mathrm{kms}$ north of Macuelizo and $8 \mathrm{kms}$ west of Dipilto, form part of an area of about 150,000 hectares of generally open pine forest which stretches more than $70 \mathrm{kms}$ roughly east-west along the cordillera and extends northwards to the pine forests of Honduras. Rainfall increases progressively eastwards and in this direction the lower slopes below 800 metres elevation are increasingly occupied by $\underline{P}$. caribaea and the higher slopes above 1,500 metres by $\underline{P}$. pseudostrobus and $\bar{b}$ roadleaf forest. At the western end, in the Macuelizo area, the lower slopes and valleys support only a dry thorn scrub and the only pine species is $\underline{P}$. oocarpa which in fact forms most of the pine forest throughout the cordillera.

Soil: Very freely-draining sandy or gravelly soil with abundant quartz, derived in situ from decaying granitic rocks, outcropping extensively on the steeper slopes and ridges. Erosion is active. The soils are generally very shallow, except in hollows or valleys, and have very little humus content. pH 5.7 .

Climate: Mean annual rainfall at Macuelizo ( $5 \mathrm{~km} \mathrm{~S}$ ) is $904 \mathrm{~mm}$ (35 ins) with the following distribution:-

$\begin{array}{cccccccccccc}\mathrm{J} & \mathrm{F} & \mathrm{M} & \mathrm{A} & \mathrm{M} & \mathrm{J} & \mathrm{J} & \mathrm{A} & \mathrm{S} & 0 & \mathrm{~N} & \mathrm{D} \\ 4 & 1 & 21 & 16 & 113 & 180 & 110 & 85 & 149 & 163 & 49 & 13\end{array}$

No temperature data are available from Macuelizo but at 0 cotal, about $20 \mathrm{kms}$ east and 400 metres below the level of E1 Junquillo mean monthly maximum temperatures in the dry season range from $28^{\circ} \mathrm{C}$ to $32^{\circ} \mathrm{C}$.

Description of stand: Very open pine forest on steep slopes ( $20^{\circ}$ to $35^{\circ}$ ) with only thin grass cover including Andropogon sp. and Pennisetum sp. A few Quercus sp. occur in some moister valleys. The largest pines are over 30 metres (100 ft) high and up to $80 \mathrm{cms}$ d.b.h. Pine regeneration is generally sparse although good in patches and the area shows evidence of frequent fierce fires over a long period of years. Growth rings are confused and difficult to interpret but growth appears to be slow, with 3 to 5 rings per $\mathrm{cm}$.

Seed bearers: B.H. diam (cms): 40 to 60 Height (m): 25 to 30 Branching: $70^{\circ}$ to $80^{\circ}$ Boles: Straight, cylindrical, sound.

Methods of collection: From selected trees in felling sites (36 trees)

Date of collection: January, 1971, 
Appendix 2.2 Examples of seed collection data sheets

B. Danish/FAO Tree Seed Centre, Humlebaek

SEED COLLECTION DATA - DAN/FAO FOREST TREE SEED CENTRE

DAN/FAO NO:

\section{Botanical Name:}

Provisional No:

Vernacular Name:

Provenance:

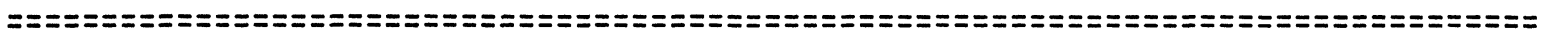

L

0

C

A

$\mathrm{L}$

I

Latitude:

Longitude:

Elevation:

Map Ref:

Detailed Location:

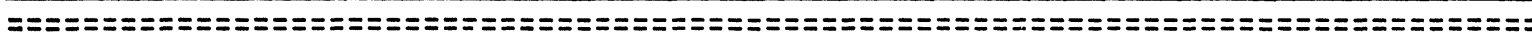

Soil Type:

Slope:

Aspect:

Drainage:

Ann. rainfall:

Monthly rainfall distrb.:

Nearest Weather Station:

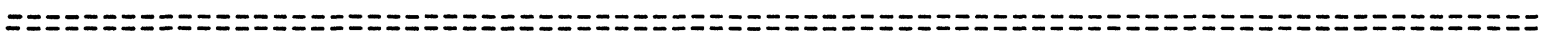

Assocation:

$\mathrm{S}$

$\mathrm{T}$

A

$\mathrm{N}$

D

\begin{tabular}{ll}
\hline Density: open.... patchy.... dense.... & Establishment \\
\hline Height: & Age: \\
\hline Diameter: & Stem Form: \\
\hline State of Stand: & \\
\hline
\end{tabular}

\section{Remarks :}

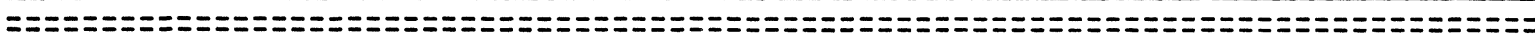

Method: Date collected:

Number of Trees:

Quantity of seed/cones: Spacing of Trees:

Condition of seed/cones:

Possibility of commercial coll.:

\section{Remarks :}

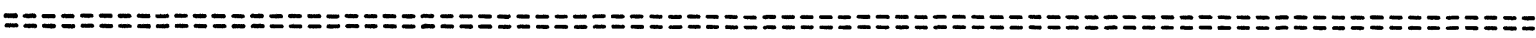

Extraction Method:

Treatment:

Yield per unit volume:

Germination:

Remarks :

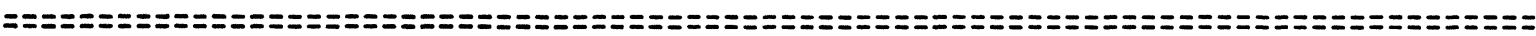




\section{Appendix 3. Site assessment methods}

\section{Appendix 3.1. Example of a land system description (Astle et al., 1969) \\ (The permission of Blackwell Scientific Publication, Ltd., to reproduce this page is gratefully acknowledged)}

A variable nearly flat landscape in which rivers branch into a number of winding channels separating broad interfluves of old alluvium. Sinuous strips of distinct soil and vegetation mark the positions of former channels. Relief a few metres only.

Land

Channel. Sinding, irregular in plan; $5-50 \mathrm{~m}$ wide with setitly sloping banks and flat or concave floor.

2 Cl.ay interfluye. Flat, extensive.

3 Sand strip. Flat; ribbon-like in plan and usually c. $500 \mathrm{~m}$ wide; with large ternite mound (4 m high).

4 SAND INTER FLUVE. Flat; irregular in plan with diffuse boundaries to facet $3 ; 1-5 \mathrm{~km}$ across.

5 SOLONETZ INTERFLUVE

(a) Level to very gently sloping (up to $1^{\circ}$ ) slightly convex; extensive $(1-7 \mathrm{~km}$ across); with numerous low mounds $(30 \mathrm{~cm}$ high and $5 \mathrm{n}$ across).

(b) As (a) above but without mounds, occurring as small pockets (c. 5 ha) within (a).
Soils, materials and hydrology

Variable, more or less stratified, sand to sandy clay loam, usually mottled. Water-logged for all or part of the year. In any one channel water flow varies greatly from year to year depending on the position of the river feeding it.

Dark mottled cracking clays or silty clays (vertisols), frequently calcareous and sodium influenced. Waterlogged during wet season and occasionally under water for a few days.

Stratified coarse and fine sand with water-worn pebbles at depth. Freely drained.

Stratified coarse and fine sand ociasionally with small rounded pebbles. Freely drained.

$10-15 \mathrm{~cm}$ of greyish-brown sand over very hard compact alkaline sandy loam or sandy clay loam, calcareous (Solonetz)

0-3 cm of greyish-brown sand over compact alkaline sandy clay loam, calcarcous (Solonetz). Drainage impeded.

\section{Vegetation}

Single tree wide strip along the banks, Adina microcephala, Diospyros mespiliformis, Trichilia emetica, Khaya nyasica. Grassland in the channel, Oryza/Echinochlva spp.

Mopane woodland with short Echinochloa grass cover. On wetter sites trees absent and watergrass (Oryza) associations present.

Woodland savanna, Erythrophloeum africanum, Terminalia sericea, Cassia abbreviata and Pseudoluchnostylis maprouneifolia, with a good grass cover of Digitaria gayana, Andropogon amplectens, Hyporrhenia eylesii, Tristachya superba. tens, Hyparrhenia eylesii, Tristachsa superba.
Combretum shrubs and Mopane occur on the mounds.

Deciduous thicket, Combretum obovatum, Diospyros senensis, D. quiloensis, Holarrhena febrifuga Markhamia acuminata, Tricalysia allenii.

Tall Mopane woodland with occasional shrubs, Combretum obovatum, $C$. elueagnoides and Holarrherka, mainly confined to the mounds, and short grass cover of Setaria pallidefusca, and Panicum hippothrix.

Scrub Mopane and sparse cover of unnual grasses, Sporobolus, Eragrostis and Chloris spp.

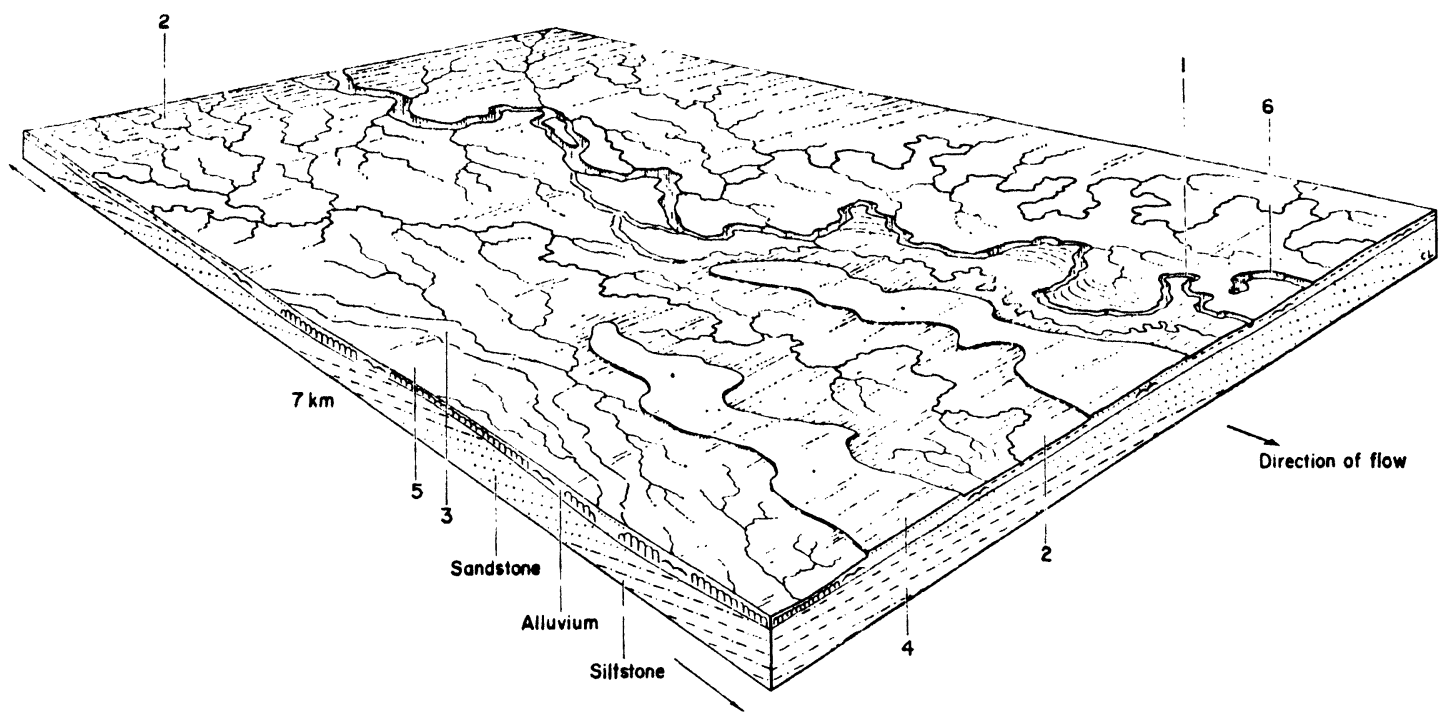

Fic. 2. Land system 2, distributary alluvial area. 
Appendix 3.2. Kuchler's (1949) scheme of vegetation description

Kuchler himself describes his scheme as a physiognomic classification of vegetation. This is not strictly true as in his scheme it is possible to have many different descriptions, all of which will have the same general appearance and thus constitute one physiognomic class. It is more correct to regard the system as a means of vegetation description. The following categories are used:-

\section{CAPITAL LETTERS}

Woody vegetation:-

B : evergreen broadleaf

D : deciduous broadleaf

$E$ : evergreen needleleaf (coniferous)

$\mathrm{N}$ : deciduous needleleaf (coniferous)

0 : without leaves

Herbaceous vegetation:-

G : graminoids

$\mathrm{H}$ : forbs

$\mathrm{L}$ : 1ichens and mosses

SMALL LETTERS:-

Group I : Height:-

$t$ : tall, minimum height of trees : $25 \mathrm{~m}$ minimum height of herbaceous

m : medium tal1; trees: $10-25 \mathrm{~m}$

herbaceous plants: $0.5-2 \mathrm{~m}$

1 : low; maximum height of trees: $10 \mathrm{~m}$ maximum height of herbaceous plants: $0.5 \mathrm{~m}$

8 : shrubs, minimum height: $1 \mathrm{~m}$

z : dwarf shrubs; maximum height: $1 \mathrm{~m}$

Group II : Density:-

c : continuous growth

i : plants usually do not touch

$\mathrm{p}$ : woody plants scattered singly or in groves herbaceous plants in disconnected patches

$r$ : rare, yet conspicuous

b : barren; vegetation largely or entirely absent

Group III : Special features:-

e : epiphytes 
j : lianas

$\mathrm{k}$ : succulents

$\mathrm{q}:$ cushion plants

$\mathrm{n}$ : palms

$\mathrm{v}$ : bamboos

$w$ : aquatic vegetation

$y$ : tree ferns and tuft plants

Using this system a larch stand with semi-deciduous ericaceous shrubs and a continuous ground cover of sedges resting on Sphagnum moss, is denoted by Nmi. Dzi. Glc. Lc. the use of periods to separate the synusiae is important to ensure clarity. Each segment of the formula should contain one capital letter and one letter each of groups I and II, with the exception of group III features when they occur.

This method is preferable to one involving fixed classes. Allowance is made for all possible combinations without the inevitable confusion that would arise if an attempt was made to pre-define those combinations.

In this Chapter the system is presented in tabulated checklist form to facilitate application in the field. Provision is also made for adding information on the floristic composition of each synusia, which should be regarded as a necessary option if the required expertise is available.

This presentation will accommodate the derivation of classifications using one or more of items 1 to 4 previously described in the 1 ist of data categories. Separate notes on items 5 to 7 , i.e. dynamics, habitat, and history, can be included if it is possible to make the necessary deductions. 
Appendix 3.2 Form 1

VEGETATION DESCRIPTION

Woody Vegetation

\begin{tabular}{|c|c|c|c|c|c|}
\hline . & $\begin{array}{c}\text { B } \\
\text { evergreen } \\
\text { broadleaf }\end{array}$ & $\begin{array}{c}\text { D } \\
\text { deciduous } \\
\text { broadleaf }\end{array}$ & $\begin{array}{c}E \\
\text { evergreen } \\
\text { needleleaf } \\
\text { (conifer- } \\
\text { ous) }\end{array}$ & $\begin{array}{c}\mathrm{N} \\
\text { deciduous } \\
\text { needleleaf } \\
\text { (conifer- } \\
\text { ous) }\end{array}$ & $\begin{array}{c}0 \\
\text { without } \\
\text { leaves }\end{array}$ \\
\hline \multicolumn{6}{|l|}{ Height:- } \\
\hline \multicolumn{6}{|l|}{$\begin{array}{l}\mathrm{t}: \text { tal1; } \\
\text { minimum ht. } 25 \mathrm{~m}\end{array}$} \\
\hline \multicolumn{6}{|l|}{$\begin{array}{l}\text { m: medium } \\
10-25 \mathrm{~m}\end{array}$} \\
\hline \multicolumn{6}{|l|}{$\begin{array}{l}\text { 1: low } \\
\text { max. ht. } 10 \mathrm{~m}\end{array}$} \\
\hline \multicolumn{6}{|l|}{ s: min. ht. $1 \mathrm{~m}$} \\
\hline \multicolumn{6}{|l|}{$\mathrm{z}: \max . \mathrm{ht} .1 \mathrm{~m}$} \\
\hline \multicolumn{6}{|l|}{ Density:- } \\
\hline \multicolumn{6}{|l|}{$\begin{array}{l}\text { c: } \text { continouus } \\
\text { growth }\end{array}$} \\
\hline \multicolumn{6}{|c|}{$\begin{array}{l}\text { 1: plants usually do } \\
\text { not touch }\end{array}$} \\
\hline \multicolumn{6}{|l|}{$\begin{array}{l}\text { p: scattered singly } \\
\text { or in groves }\end{array}$} \\
\hline \multicolumn{6}{|l|}{$\begin{array}{l}r: \text { rare, yet } \\
\text { conspicuous }\end{array}$} \\
\hline Principal species & & & & & \\
\hline
\end{tabular}


Appendix 3.2 Form 2

VEGETATION DESCRIPTION

Herbaceous Vegetation

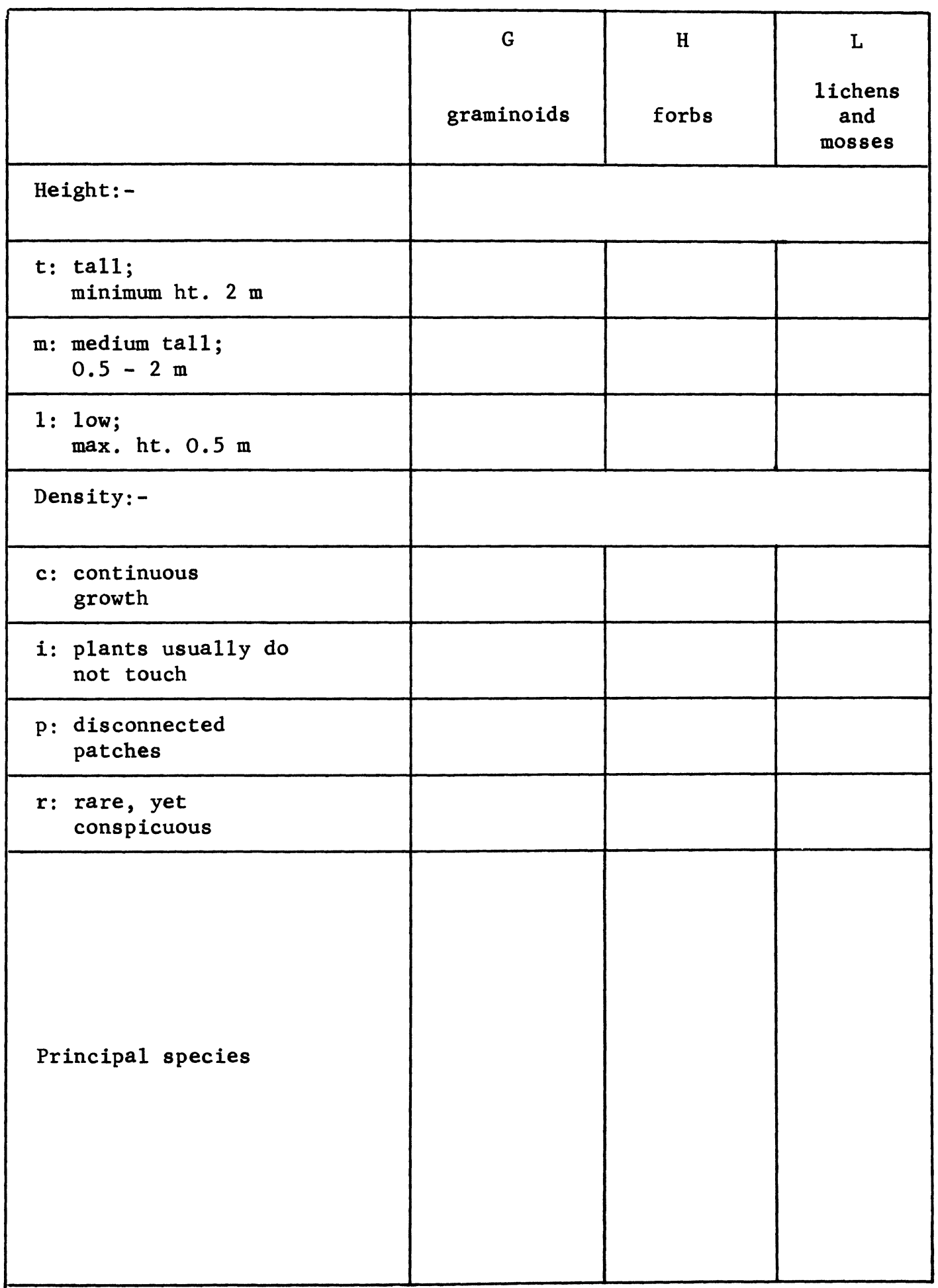


VEGETATION DESCRIPTION

Special Features

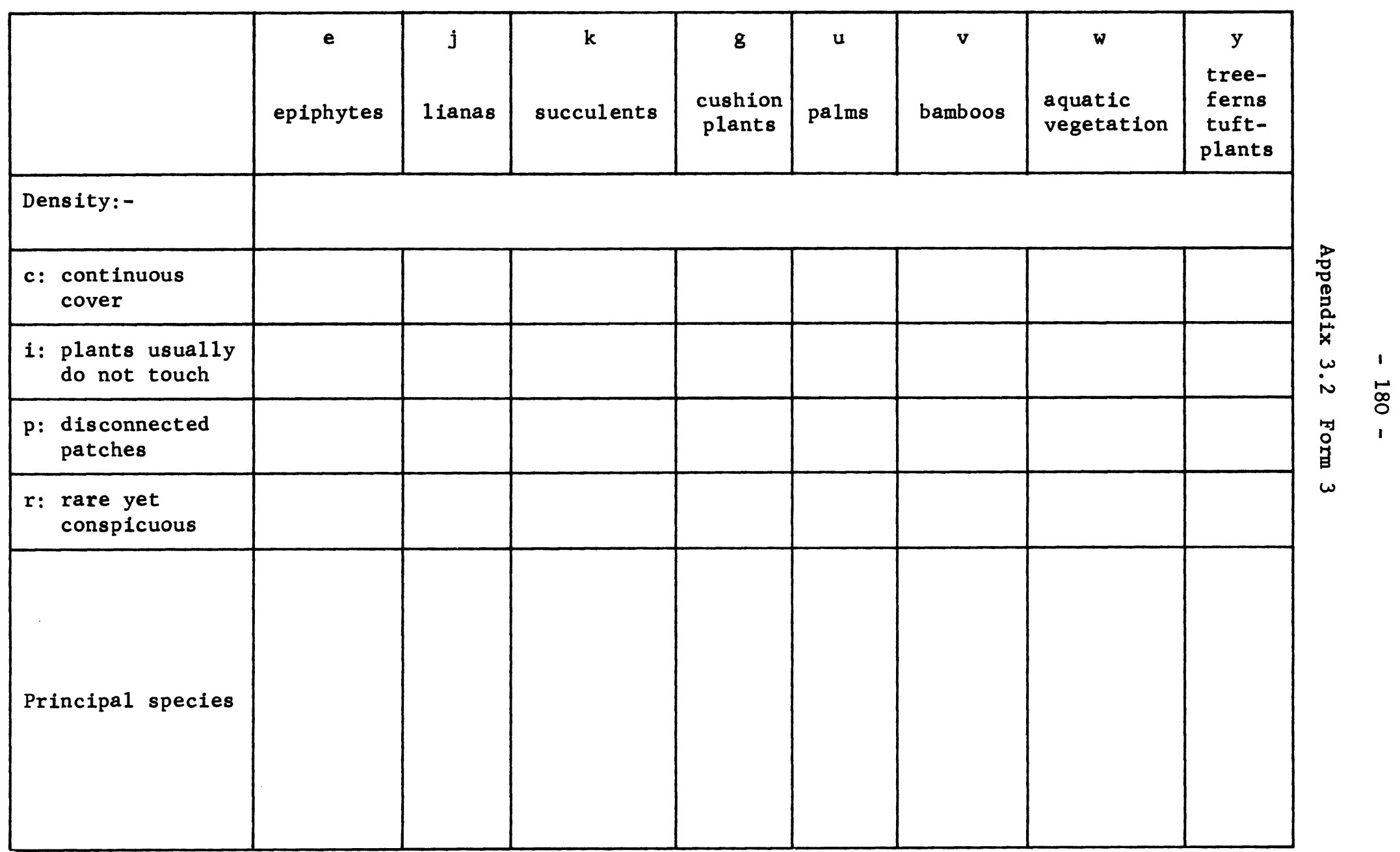


SOIL PROFILE DESCRIPTION (after Jenkin, 1963)

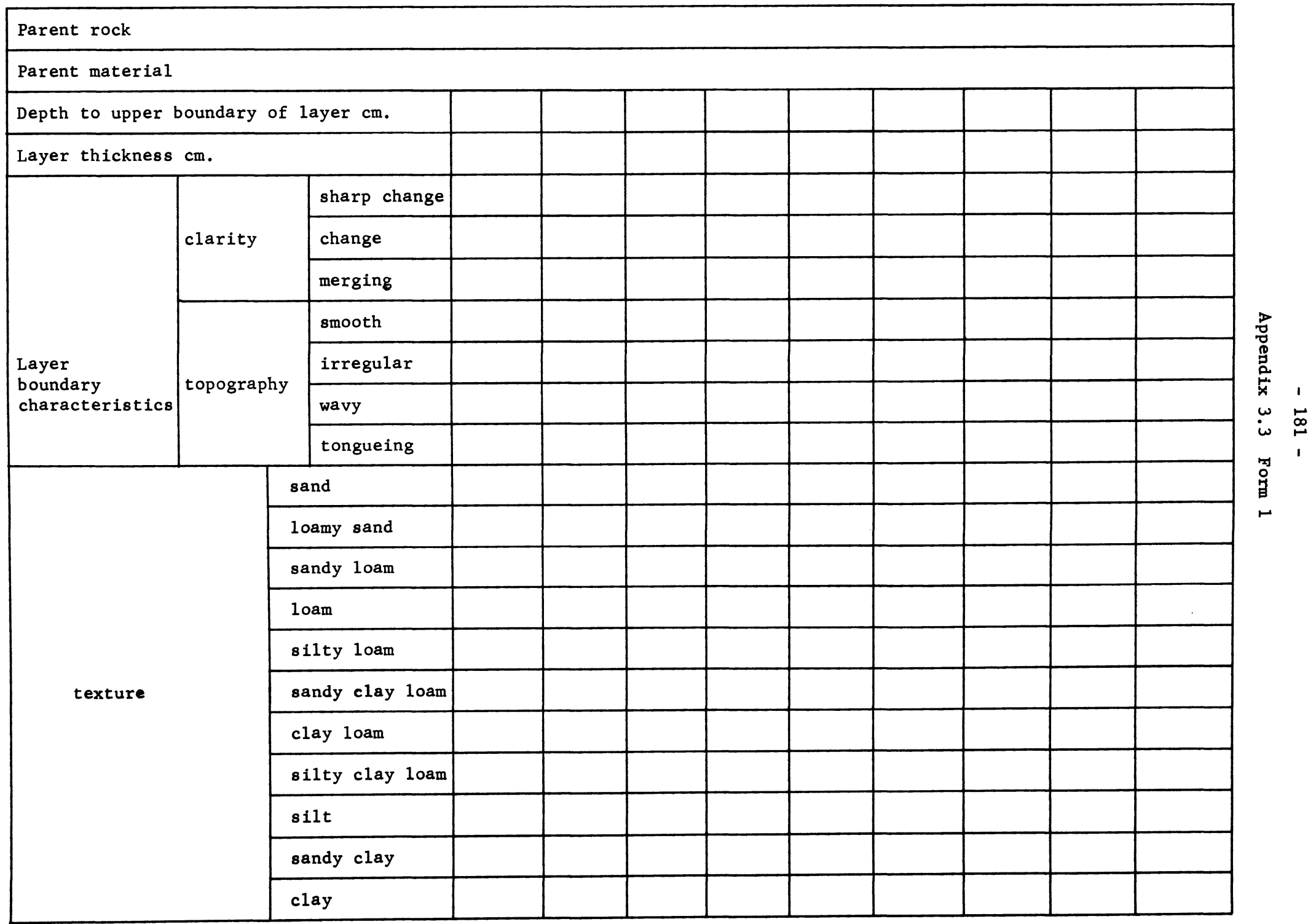


SOIL PROFILE DESCRIPTION

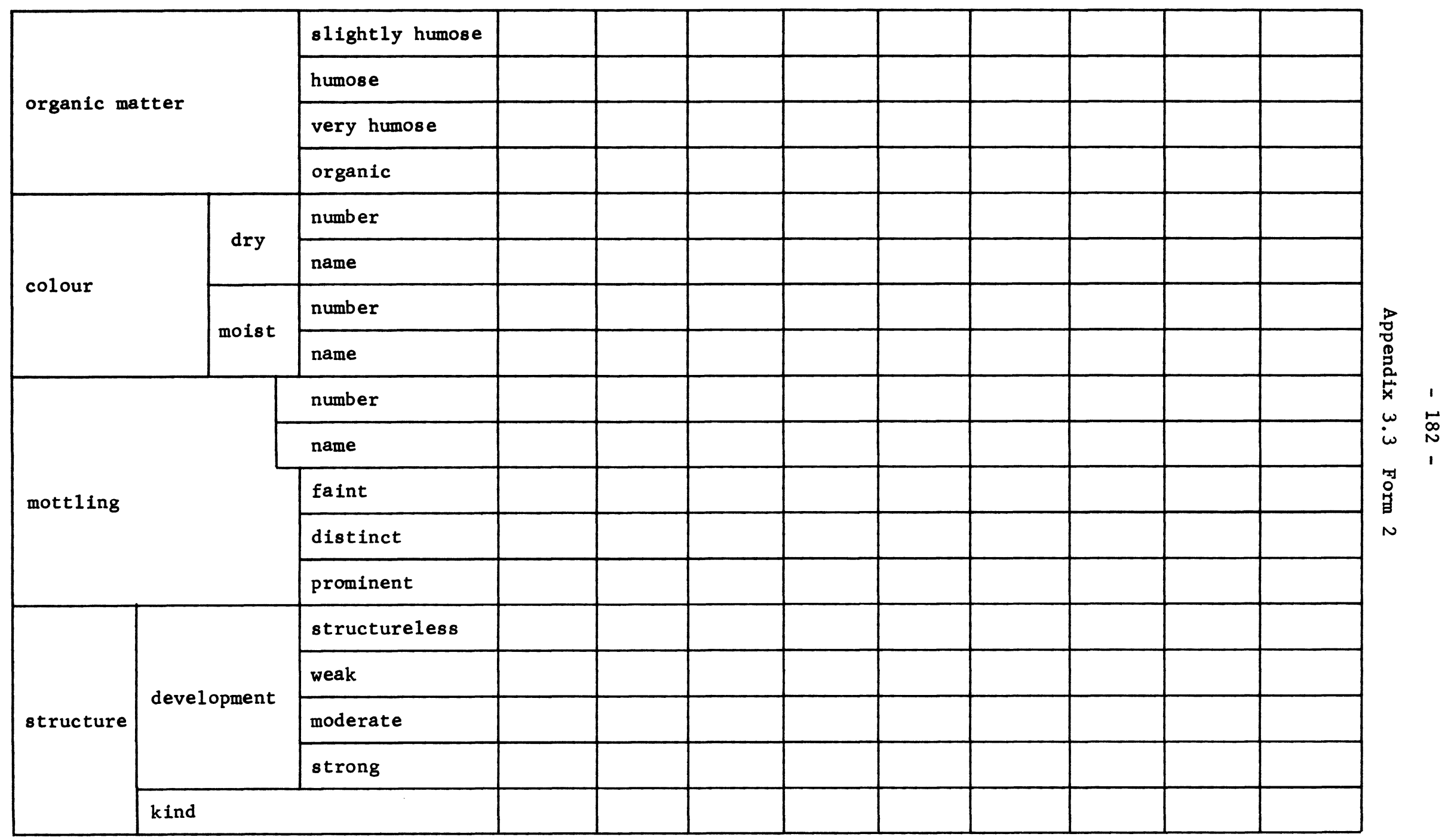


SOIL PROFILE DESCRIPTION

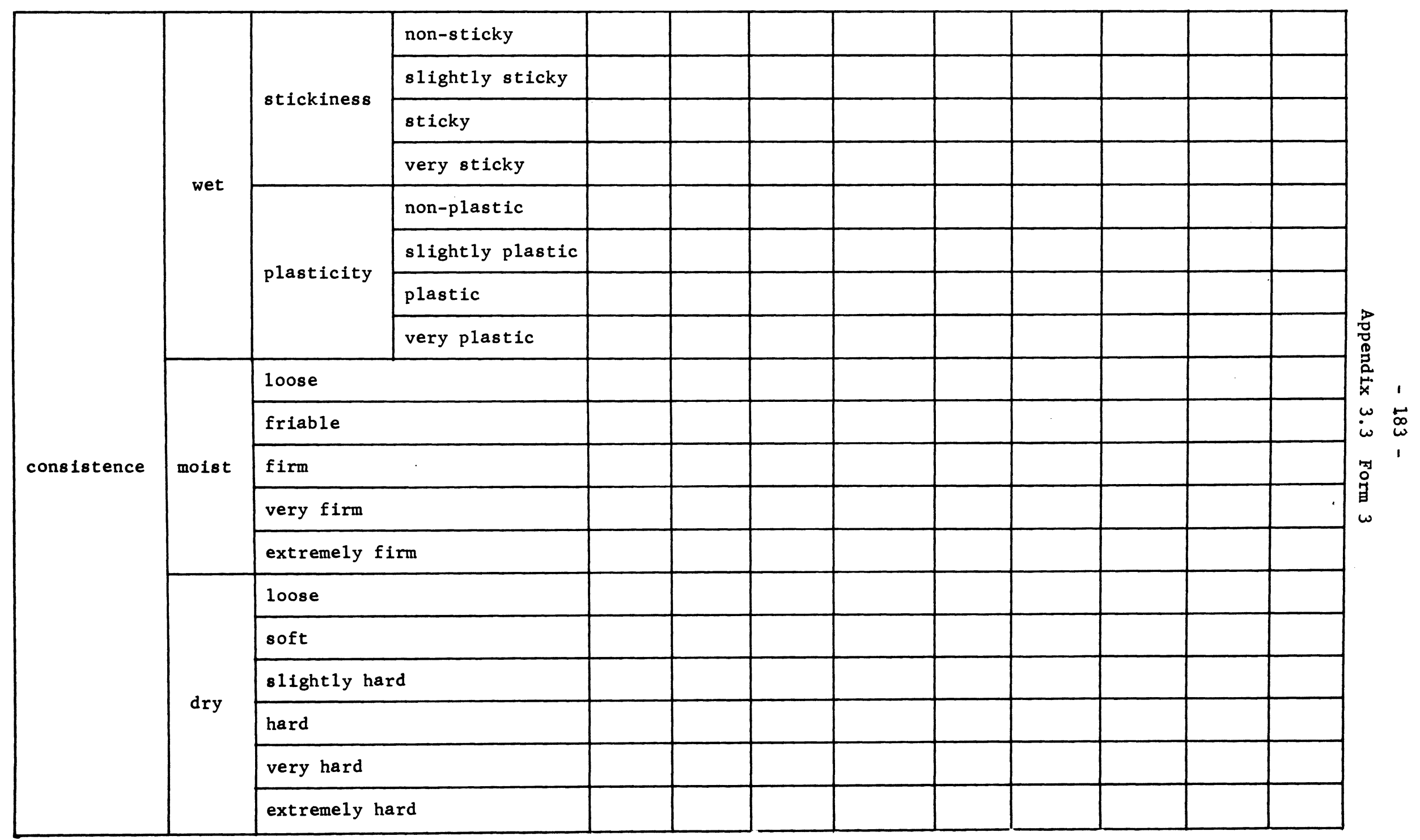


Appendix 3.4 Climatic data and estimation of potential evapotranspiration and the computation of water balances

A. The estimation of potential evapotranspiration by Thornthwaite's method

\section{Data required}

\section{Mean monthly temperatures}

Latitude

\section{Procedure}

There are three stages in the procedure for the estimation of potential evapotranspiration and tables to facilitate the calculation have been published by Thornthwaite and Mather (1957).

1) The first stage is to obtain the Heat Index (I) from Thornthwaite's tables 1 or 2, which give monthly values (1) for this factor corresponding to the mean monthly temperatures in ${ }_{F}$ or ${ }^{\circ}$.

2) The second stage is to determine, from Thornthwaite's tables 3 and 4, the unadjusted daily potential evapotranspiration for the mean monthly temperatures and monthly values of the Heat Index (I) recorded.

3) Finally, the daily values for potential evapotranspiration are adjusted for differences in the mean daylength of each month, from correction factors given in Thornthwaite's tables 6 and 7 .

An example is given in Table 1 of this Appendix.

Table 1

Example of estimation of potential evapotranspiration; Zomba Plateau, Malawi (160 1 latitude)

\begin{tabular}{|r|c|c|c|c|c|}
\hline${ }_{\mathrm{o}_{\mathrm{f}_{\mathrm{h}}}}$ & $\begin{array}{c}\text { Mean monthly } \\
\text { temp. }\left({ }^{\mathrm{C}} \mathrm{C}\right)\end{array}$ & $\begin{array}{c}\text { Monthly (i) and } \\
\text { annual }(\mathrm{I}) \text { index }\end{array}$ & $\begin{array}{c}\text { Unadj. daily } \\
\mathrm{PE}(\mathrm{mm})\end{array}$ & $\begin{array}{c}\text { Correction } \\
\text { factors }\end{array}$ & $\mathrm{PE}(\mathrm{mm})$ \\
\hline $\mathrm{J}$ & 18.1 & 7.01 & 2.4 & 33.6 & 81 \\
$\mathrm{~F}$ & 17.9 & 6.90 & 2.4 & 29.7 & 71 \\
$\mathrm{M}$ & 17.3 & 6.55 & 2.2 & 31.5 & 69 \\
$\mathrm{~A}$ & 16.4 & 6.04 & 2.0 & 29.4 & 59 \\
$\mathrm{M}$ & 14.2 & 4.86 & 1.7 & 29.4 & 50 \\
$\mathrm{~J}$ & 12.7 & 4.10 & 1.4 & 27.9 & 39 \\
$\mathrm{~J}$ & 12.2 & 3.86 & 1.2 & 29.1 & 35 \\
$\mathrm{~A}$ & 14.0 & 4.75 & 1.6 & 30.0 & 48 \\
$\mathrm{~S}$ & 16.5 & 6.10 & 2.0 & 30.0 & 60 \\
$\mathrm{O}$ & 19.2 & 7.67 & 2.6 & 32.1 & 83 \\
$\mathrm{~N}$ & 19.4 & 7.79 & 2.7 & 32.1 & 87 \\
$\mathrm{D}$ & 18.3 & 7.13 & 2.4 & 33.9 & 81 \\
$\mathrm{Y}$ & - & 72.76 & - & - & 763 \\
\hline
\end{tabular}




$$
\begin{aligned}
& E_{0}=\frac{\Delta}{\Delta+Y} \cdot\{\operatorname{Ra}(1-r)(a+b n / N\} \quad \text { (incoming shortwave radiation) } \\
& -\frac{}{\Delta+\mathrm{Y}}\left\{\mathrm{Ta}^{4}\left(0.56-0.092 \mathrm{e}^{\prime}\right)(0.10+0.90 \mathrm{n} / \mathrm{N}\} \text { (outgoing 1ongwave radi- } \begin{array}{l}
\text { ation) } \\
\text { ation }
\end{array}\right. \\
& +\frac{Y}{\Delta+Y} \quad 0.35(1+u / 100)\left(e-e^{\prime}\right) \quad \text { (bulk aerodynamic term) }
\end{aligned}
$$

in which:-

$\mathrm{E}_{\mathrm{O}}=$ potential evaporation in $\mathrm{mm}$ per day

$\Delta=$ increase in saturation vapour pressure per unit increase in temperature

$\mathrm{Y}=$ psychometric constant in $\mathrm{MB}$ per ${ }_{\mathrm{C}}$

$\mathrm{Ra}=$ incoming shortwave radiation in cal./sq. cm/day

$\mathrm{Ta}^{4}=$ black body radiation at mean air temperature

$\mathrm{e}^{\prime}=$ vapour pressure at mean dew point in mb

$\mathrm{n}=$ hours of actual sunshine per day

$\mathrm{N}=$ Hours of possible sunshine per day

$\mathrm{u}=$ run of wind at $2 \mathrm{~m}$ height, in miles per day

$\mathbf{e}_{\mathbf{a}}=$ saturated vapour pressure at mean dry bulb temperature in mb

Programmes are available to carry out the computation (McCulloch, 1965), and this is commonly done on either a monthly or on a 10 day basis. For tropical East Africa the relative magnitude of the three terms are roughly in the ratio $4: 1: 1$.

Unfortunately, very few meteorological stations measure all the variables required for the computation of potential evaporation from open water ( $\left.E_{0}\right)$, and in particular measurements of radiation, a major factor in the computation, are lacking. Because of this situation various techniques have been developed for the estimation of the meteorological parameters not recorded, of which the most useful is based upon the relationships between the amount of cloud, the duration of sunshine and the insolation (Pereira, Dagg and Hosegood, 1962; Woodhead, 1968; Rijks, Owen and Hanna', 1970).

A further complication is that few forest meteorological stations will have the information on cloud and the duration of sunshine, etc., required for these estimations, so that values of $\mathrm{E}_{0}$ for forest areas have to be derived from other stations. Maps showing the monthly values for potential evaporation from open water $\left(E_{o}\right)$ can be compiled from monthly values of $E_{o}$, after taking into account the effect of altitude. However, it should be recognized that because of the restricted distribution of meteorological stations and the fact that potential evaporation from open water can change considerably over short distances particularly in mountainous areas, there may be considerable errors in values obtained by interpolation from maps.

Penman's $E_{0}$ is an estimate of the potential evaporation from open water and to be of practical use it has to be converted to an estimate of actual 
evaporation and transpiration from forest plantations. This requires the measurement of water used at the particular site, as was carried out in East Africa, for example, by Pereira and Hosegood (1962) and Pereira, Dagg and Hosegood (1962). As already indicated, the conversion factor to obtain an estimate of actual evapotranspiration may vary considerably from site to site and also at different times during the year, particularly at sites with shallow soils and long dry seasons. Although it is generally recognized that a more accurate estimation of evapotranspiration is likely to be obtained than with alternative methods, the work involved in determining conversion factors, together with a lack of meteorological data, will limit at present the application of Penman's method for forestry research in the majority of tropical countries. Because of these difficulties the computation of the water balance by Thornthwaite's method is described here which requires only the climatic data likely to be available at most forest stations.

\section{B. The computation of the water balance according to Thornthwaite's method}

The term water balance as used here refers to the differences between the intake of water at a site from precipitation and the outflow by evaporation, transpiration and run off.

The water balance can be obtained on a daily, weekly or monthly basis. For the purpose of tree species and provenance research, a calculation on a monthly basis is suggested, because in most countries, the information will be available to do this on the territorial or regional scale that is required if it is to be of practical use. Computation on a monthly basis will result in underestimation of water deficiencies or surpluses, but this is unlikely to cause serious mistakes in the comparison of conditions at different sites.

In the computation of a water balance there are six main items - the potential evapotranspiration ( $P E)$, the precipitation $(P)$, the soil moisture storage (ST), the moisture deficit (D) and the moisture surplus (S).

Calculation of the balance is done as shown in the example given in Table 2 in this Appendix.

\section{Starting point}

For "wet" stations, that is where annual precipitation ( $P$ ) exceeds the annual potential evapotranspiration ( $P E)$, the easiest procedure is to go back to the end of the previous wet season, where soil moisture storage (ST) has reached its maximum estimated value for the site, and to work from that point onwards to determine the value for soil moisture storage (ST) for January of the year for which the water balance is required.

If rainfall data is not available for the previous year, and also for "dry" stations where soil moisture storage (ST) does not normally reach its potential maximum value, methods have been described by Thornthwaite and Mather (1957) to obtain a value for soil moisture storage (ST) with which to start the computation of the water balance.

\section{Line 1}

The potential evapotranspiration ( $\mathrm{PE})$, calculated as explained in 
previous sections, is entered on the first line in mm for each month separately.

\section{Line 2}

The precipitation $(P)$ is entered on the second line in $\mathrm{mm}$ for each month separately.

Line 3

The monthly values for potential evapotranspiration ( $P E$ ) are then subtracted from the corresponding monthly values for precipitation $(P)$ and the result $(P-P E)$ is entered on the third line. A negative value for ( $P-P E)$ shows the amount by which the precipitation fails to meet the potential need for water in that month. A positive value for $(P-P E)$ indicates the amount of excess water from precipitation that is available for recharging soil moisture or that will be lost as run off.

In tropical countries positive and negative values for ( $P$ - PE) usually occur in sets of months corresponding to the wet and dry seasons.

\section{Line 4}

If the soil moisture storage (ST) had reached its maximum estimated value at the end of the wet season ( $300 \mathrm{~mm}$ in this example), the first negative value for precipitation minus potential evapotranspiration ( $P-P E)$ is entered as potential water loss (PWL) for that month on the fourth line.

If the soil moisture storage (ST) had not reached its maximum estimated value in the month preceding the occurrence of a negative value of $(P-P E)$, the estimated value for soil storage (ST) in that month is converted to potential water loss (PWL) from tables provided by Thornthwaite and Mather (1957) and this converted value is included in the accumulative total of potential water loss ( $\mathrm{PWL}$ ) for subsequent months.

\section{Line 5}

Where soil moisture storage (ST) has reached its maximum estimated value in the previous wet season ( $300 \mathrm{~mm}$ in the example), the first monthly value for potential water loss (PWL - $27 \mathrm{~mm}$ for the month of May in the example) is converted to an equivalent value for soil moisture storage (274 $\mathrm{mm}$ for soil of a maximum capacity of $300 \mathrm{~mm}$ in the example) from tables provided by Thornthwaite and Mather (1957) and is entered on line 5.

If precipitation minus potential evaporation $(P-P E)$ for the next month is positive ( $+9 \mathrm{~mm}$ for the month of June in the example), this value is added to the soil moisture storage for the previous month and entered on line $4(274+9=283 \mathrm{~mm}$ for the month of June).

If the following month (July in the example) has a negative value for precipitation minus potential evapotranspiration $(P-P E)$, and soil storage (ST) is still not at its maximum value $(300 \mathrm{~mm})$, the value for soil moisture storage of the previous month (283 mm for the month of June) is converted to 
an equivalent value for potential water loss (PWL) from Thornthwaite's tables and entered in line 4 (ST of $283 \mathrm{~mm}=$ PWL of $-17 \mathrm{~mm}$, entered for the month of June). It is then added to the current negative value for precipitation minus potential evapotranspiration $(P-P E)$ and entered on line 5 as potential water loss ( $-17 \mathrm{~mm}$ for PWL in June plus $-40 \mathrm{~mm}$ for $(P-P E)$ in July $=-57$ PWL for Ju1y).

As long as negative values for precipitation minus potential evapotranspiration ( $P$ - PE) occur these are accumulated in the monthly entries for potential water loss (PWL) on line 4, and the corresponding values for soil moisture storage (ST) are ascertained from Thornthwaite's tables and entered on line 5 (for all the months August to October in the example).

For the first month in which the value for precipitation minus evapotranspiration $(P-P E)$ is positive ( 331 for October in the example), this value is added to soil moisture storage of the previous month and entered on line 5 .

If, however, this value exceeds the maximum soil moisture storage capacity $(300 \mathrm{~mm})$, then the maximum value only is entered on 1 ine 5 and the excess ( $199 \mathrm{~mm}$ in the example) is entered as surplus ( $S$ ) on line 9. As long as the value for precipitation minus evapotranspiration $(P-P E)$ is positive the maximum value for soil moisture storage (ST) is entered on line 5 (to the month of December in the example).

\section{Line 6}

The difference to the value for soil moisture storage (ST) when compared with the value for the previous month is entered on the sixth line ( $300 \mathrm{~mm}-74 \mathrm{~mm}=-26 \mathrm{~mm}$ for the month of May in the example).

Negative values for these differences in soil moisture storage are required for computation of actual evapotranspiration ( $A E$ ) for the months with negative values for precipitation minus potential evapotranspiration $(P-P E)$.

\section{Line 7}

Actual evapotranspiration ( $A E)$, which is entered on the seventh 1 ine, is equal to potential evapotranspiration $(\mathrm{PE})$ for months with positive values for precipitation minus potential evapotranspiration ( $P$ - $P E$ ) and also when precipitation is equal to potential evapotranspiration ( $P=P E$ ) (the months January to April, June, November and December in the example).

For the dry months, in which potential evapotranspiration exceeds precipitation ( $P E>P$ ), the actual evapotranspiration ( $A E$ ) is equal to precipitation ( $P$ ) plus the change in soil moisture storage ( $\Delta \mathrm{ST}$ ), but disregarding its sign (it is therefore $44 \mathrm{~mm}+26 \mathrm{~mm}=70 \mathrm{~mm}$ for the month of May, and $16 \mathrm{~mm}+35 \mathrm{~mm}=51 \mathrm{~mm}$ for the month of July in the example).

\section{Line 8}

For months in which actual evapotranspiration is equal to potential 
evapotranspiration $(\mathrm{AE}=\mathrm{PE}$ ) there is no deficit (D) and zero is entered on the eighth line.

If the actual evapotranspiration $(\mathrm{AE})<\mathrm{PE}$, the difference is entered on line 8 as a deficit (D) for that month $(71 \mathrm{~mm}-70 \mathrm{~mm}=1 \mathrm{~mm}$ for May and $56 \mathrm{~mm}$ - $51 \mathrm{~mm}=5 \mathrm{~mm}$ for July in the example).

\section{Table 2}

Example of computation of water balance (Thornthwaite); Ukaguru, Tanzania Months

\begin{tabular}{|c|c|c|c|c|c|c|c|c|c|c|c|c|c|}
\hline & $\mathrm{J}$ & F & M & A & M & $\mathrm{J}$ & $\mathrm{J}$ & A & S & 0 & $\mathrm{~N}$ & $\mathrm{D}$ & $\mathrm{Y}$ \\
\hline \multicolumn{6}{|c|}{ Station: Ukaguru } & & & & & & & & \\
\hline $\mathrm{PE}$ & 91 & 86 & 90 & 81 & 71 & 60 & 56 & 59 & 66 & 75 & 86 & 88 & 909 \\
\hline $\mathbf{P}$ & 203 & 152 & 209 & 266 & 44 & 69 & 16 & 17 & 47 & 20 & 417 & 144 & 1604 \\
\hline $\mathrm{P}-\mathrm{PE}$ & +112 & +66 & +119 & +185 & -27 & +9 & -40 & -42 & -19 & -55 & +331 & +56 & +695 \\
\hline Acc. PWL & & & & & -27 & $(-17)$ & -57 & $-99 \mid$ & -118 & -173 & & & \\
\hline ST & 300 & 300 & 300 & 300 & 274 & 283 & 248 & 215 & 202 & 168 & 300 & 300 & \\
\hline ST & 0 & 0 & 0 & 0 & -26 & +9 & -35 & $-33 \mid$ & -13 & -34 & +132 & 0 & \\
\hline $\mathrm{AE}$ & 91 & 86 & 90 & 81 & 70 & 60 & 51 & 50 & 60 & 54 & 86 & 88 & 867 \\
\hline D & 0 & 0 & 0 & 0 & 1 & 0 & 5 & 9 & 6 & 21 & 0 & 0 & 42 \\
\hline S & 112 & 66 & 119 & 185 & 0 & 0 & 0 & o & 0 & 0 & 199 & 56 & 737 \\
\hline
\end{tabular}


Appendix 4. A selection of methods for determining the number of replications required

Determination of the number of replications required in a trial using a RCB design.

The number of replications needed may differ markedly for different parameters, and it is therefore essential to select the most important parameter for calculation. A rule of thumb in the planning of the analysis of variance is never to have less than 10 degrees of freedom for residual error, and preferably about 15 or more. Thus with four species or provenances one would need about 6 or more replications, thus

\begin{tabular}{|c|c|c|c|c|c|c|c|c|}
\hline Anovar 1 & $\begin{array}{l}\frac{\text { No }}{4} \\
6\end{array}$ & $\begin{array}{l}\text { Source } \\
\text { Species } \\
\text { Replications } \\
\text { Residual }\end{array}$ & 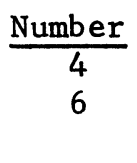 & $\begin{array}{l}\text { d.f. } \\
3 \\
5 \\
15\end{array}$ & 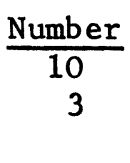 & $\begin{array}{l}\frac{\text { d.f. }}{9} \\
2 \\
18\end{array}$ & 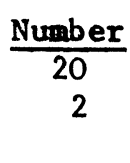 & $\begin{array}{c}\text { d.f. } \\
19 \\
1 \\
19\end{array}$ \\
\hline & 24 & Tota1 & 23 & & 30 & & 40 & \\
\hline
\end{tabular}

The effect of increasing the number of species or provenances is to decrease the number of replications required, thus

\begin{tabular}{|c|c|c|c|c|c|c|}
\hline Anovar 2 . & $\begin{array}{r}\frac{\text { No }}{10} \\
3\end{array}$ & $\begin{array}{l}\text { Source } \\
\text { Species } \\
\text { Replications } \\
\text { Residual }\end{array}$ & $\begin{array}{r}\text { d.f. } \\
9 \\
2 \\
18\end{array}$ & $\frac{\text { No. }}{20}$ & $\begin{array}{l}\text { Source } \\
\text { Species } \\
\text { Replications } \\
\text { Residual }\end{array}$ & $\begin{array}{r}\text { d.f. } \\
19 \\
1 \\
19\end{array}$ \\
\hline & 30 & Total & 29 & 40 & Total & 39 \\
\hline
\end{tabular}

This rule of thumb is based on the fact that table "F" values change comparatively little for a residual 12 degrees of freedom and above.

The number of replications required to achieve a certain probability of obtaining a significant result can also be calculated, (see Cochran and Cox, 1957, page 17). This gives a different number of replications from the rule of thumb above, because it takes account both of the variability of the material, and the size of difference that the experiment is required to detect. Clearly, if the coefficient of variation percent is greater than the percentage difference it is desired to detect, no amount of replication can enable this to be done. The ratio of the variation (standard error or coefficient of variation CV\%) to the difference required (actual value, or percentage difference) must therefore be less than 1 . The following procedures may be used.

\section{Estimation of variation}

An estimate of the standard deviation (SD) of the plot mean values is required which may be available from other trials as the square root of the residual mean square in the analysis of variance. If there is no previous information, 9 estimate can be derived from the expected range of the treatment means within a single block:-

$$
S D=f \times \text { range }
$$

" $f$ " is a factor dependent on the number of treatments, and obtained from the following table. 


\begin{tabular}{|cr|cc|}
\hline No. of treatments & $\mathbf{f}$ & No. of treatments & $\mathbf{f}$ \\
\hline 2 & 0.886 & 9 & 0.337 \\
3 & 0.591 & 10 & 0.325 \\
4 & 0.486 & 12 & 0.307 \\
5 & 0.430 & 14 & 0.294 \\
6 & 0.395 & 16 & 0.283 \\
7 & 0.370 & 18 & 0.275 \\
8 & 0.351 & 20 & 0.268 \\
\hline
\end{tabular}

Having obtained an estimated standard deviation it is necessary to decide the difference (D) to be detected using the same units (or percentages) for both values.

The ratio SD/D is then calculated. From the following table is obtained the number of replications required for a difference of the magnitude chosen to be detected as significant at the $p=0.05$ leve1. This gives a probability of $95 \%$ of the result being significant. For other levels of probability, or degrees of significance, Cochran and Cox (1966) and/or a professional biometrician should be consulted.

\begin{tabular}{|c|c|c|c|}
\hline \multirow{2}{*}{ Ratio SD/D } & \multicolumn{3}{|c|}{ Number of treatments } \\
\cline { 2 - 3 } & \multicolumn{4}{|c|}{4} & 12 & 20 \\
\hline 0.2 & \multicolumn{4}{|c|}{ Number of } & replications \\
\hline 0.3 & 2 & 2 & 2 \\
0.4 & 3 & 3 & 2 \\
0.5 & 5 & 4 & 4 \\
0.6 & 7 & 6 & 6 \\
0.7 & 9 & 8 & 8 \\
0.8 & 12 & 11 & 10 \\
& 15 & 13 & 13 \\
\hline
\end{tabular}

It will be noted that the number of replicates required varies very little with the number of treatments, but considerably with the SD/D ratio. This illustrates the extreme importance of keeping the unexplained variation in the experiment to a minimum, by making all non experimental factors as uniform as possible. This method is of little value if the ratio $\mathrm{SD} / \mathrm{D}$ is greater than 0.5 because it suggests impracticably high numbers of replications. A modified direct formula for estimating the number of replications required $(r)$ is given by

$$
r=\frac{t^{2} \times \text { range }}{8 \times L^{2} D^{2}}
$$

where LSD is least significant difference.

Determination of the number of replications required in a fully randomised trial

The number of replications for a completely randomised trial can be obtained from the table below. This table is similar to the one above in its probability and significance levels. 


\begin{tabular}{|c|c|c|}
\hline \multirow{3}{*}{ Ratio SD/D } & \multicolumn{2}{|c|}{ Number of treatments } \\
\hline & 4 & $12+$ \\
\hline & \multicolumn{2}{|c|}{ Number of replications } \\
\hline 0.2 & 2 & 2 \\
\hline 0.3 & 3 & 2 \\
\hline 0.4 & 4 & 3 \\
\hline 0.5 & 5 & 5 \\
\hline 0.6 & 7 & 6 \\
\hline 0.7 & 9 & 8 \\
\hline 0.8 & 11 & 11 \\
\hline 0.9 & 13 & 13 \\
\hline
\end{tabular}

In this it is assumed that there are equal numbers of replications per treatment. It will be noted that fewer replications are needed for a given SD/D ratio and number of treatments than for an RCB design. This is because of the larger number of degrees of freedom available in a completely randomised design. However this advantage could be offset by an increase in the expected standard deviation. 
Appendix 5. Plans for lattices with 12,16,20,25,30 and 36 treat ments

Table (a). 12 treatments: $3 \times 4$ rectangular lattice

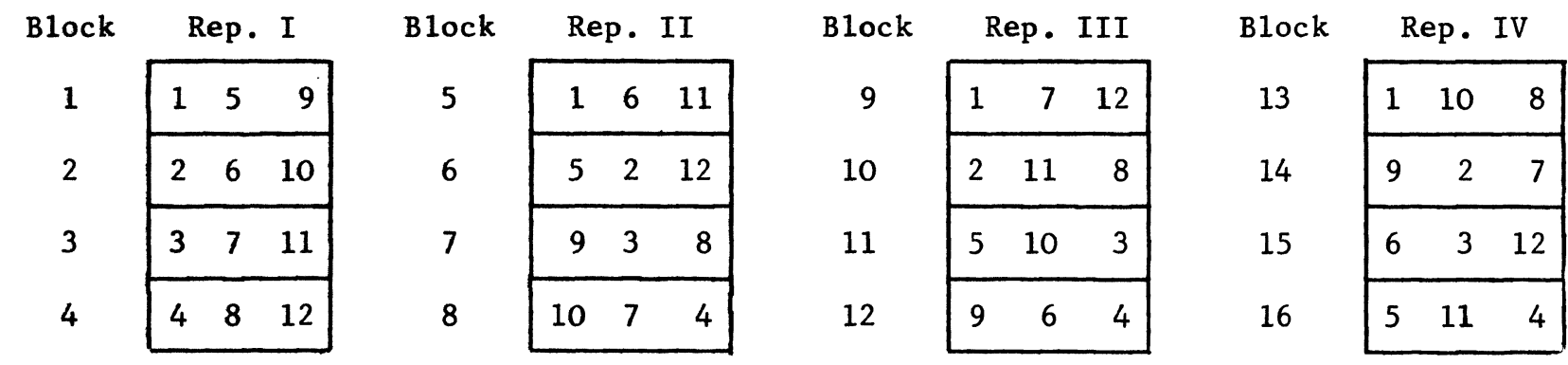

Table (b). 16 treatments: balanced $4 \times 4$ square lattice

\begin{tabular}{|c|c|c|c|c|}
\hline Block & \multicolumn{4}{|c|}{ Rep. I } \\
\hline 1 & 1 & 2 & 3 & 4 \\
\hline 2 & 5 & 6 & 7 & 8 \\
\hline 3 & 9 & 10 & 11 & 12 \\
\hline 4 & 13 & 14 & 15 & 16 \\
\hline
\end{tabular}

\begin{tabular}{|c|c|c|c|c|}
\hline & 1 & 5 & 9 & 13 \\
\hline & 2 & 6 & 10 & 14 \\
\hline & 3 & 7 & 11 & 15 \\
\hline & 4 & 8 & 12 & 16 \\
\hline
\end{tabular}

\begin{tabular}{|c|c|c|c|c|}
\hline 9 & 1 & 6 & 11 & 16 \\
\hline 10 & 5 & 2 & 15 & 12 \\
\hline 11 & 9 & 14 & 3 & 8 \\
\hline 12 & 13 & 10 & 7 & 4 \\
\hline
\end{tabular}

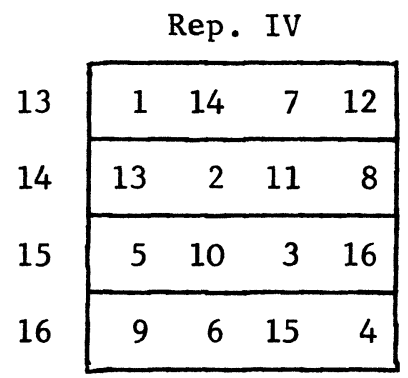

\begin{tabular}{|c|c|c|c|c|}
\hline 17 & 1 & 10 & 15 & 8 \\
\hline 18 & 9 & 2 & 7 & 16 \\
\hline 19 & 13 & 6 & 3 & 12 \\
\hline 20 & 5 & 14 & 11 & 4 \\
\hline
\end{tabular}

Table (c) 20 treatments: $4 \times 5$ rectangular lattice Rep. I Rep. II

\begin{tabular}{l|llll|}
1 & 1 & 6 & 11 & 16 \\
\cline { 2 - 4 } 2 & 2 & 7 & 12 & 17 \\
\cline { 2 - 4 } 3 & 3 & 8 & 13 & 18 \\
\cline { 2 - 4 } 4 & 4 & 9 & 14 & 19 \\
\cline { 2 - 4 } 5 & 5 & 10 & 15 & 20 \\
\hline
\end{tabular}

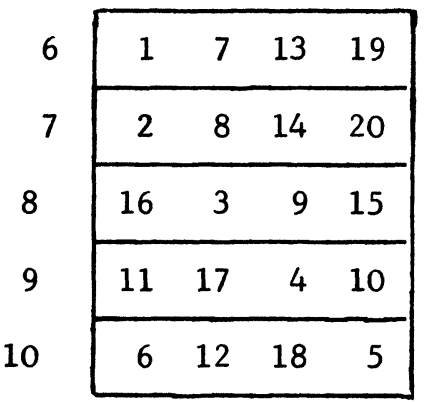

\begin{tabular}{|c|c|c|c|}
\hline 1 & 12 & 9 & 20 \\
\hline 16 & 2 & 13 & 10 \\
\hline 6 & 17 & 3 & 14 \\
\hline 7 & 18 & 4 & 15 \\
\hline 11 & 8 & 19 & 5 \\
\hline
\end{tabular}


Table (c). (Cont.)

Rep. IV

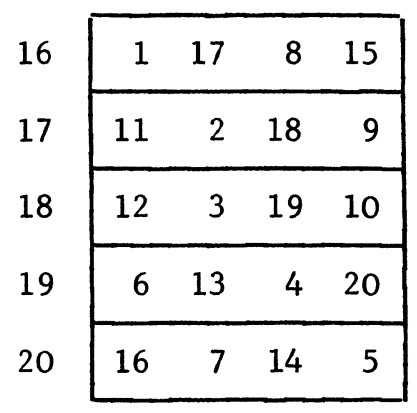

Rep. V

\begin{tabular}{|c|c|c|c|c|}
\hline 21 & 1 & 18 & 14 & 10 \\
\hline & 6 & 2 & 19 & 15 \\
\hline & 11 & 7 & 3 & 20 \\
\hline & 16 & 12 & 8 & 4 \\
\hline & 17 & 13 & 9 & 5 \\
\hline
\end{tabular}

Table (d). 25 treatments: $5 \times 5$ balanced lattice

Rep. I

\begin{tabular}{l|rrrrr|}
1 & 1 & 2 & 3 & 4 & 5 \\
\cline { 2 - 6 } 2 & 6 & 7 & 8 & 9 & 10 \\
\cline { 2 - 6 } 3 & 11 & 12 & 13 & 14 & 15 \\
\cline { 2 - 6 } & 16 & 17 & 18 & 19 & 20 \\
\hline 5 & 21 & 22 & 23 & 24 & 25 \\
\hline
\end{tabular}

Rep IV

\begin{tabular}{l|rrrrr|}
\cline { 2 - 5 } 16 & 1 & 12 & 23 & 9 & 20 \\
\cline { 2 - 5 } 17 & 16 & 2 & 13 & 24 & 10 \\
\cline { 2 - 5 } 18 & 6 & 17 & 3 & 14 & 25 \\
\cline { 2 - 5 } 19 & 21 & 7 & 18 & 4 & 15 \\
\cline { 2 - 5 } 20 & 11 & 22 & 8 & 19 & 5 \\
\hline
\end{tabular}

Rep. II

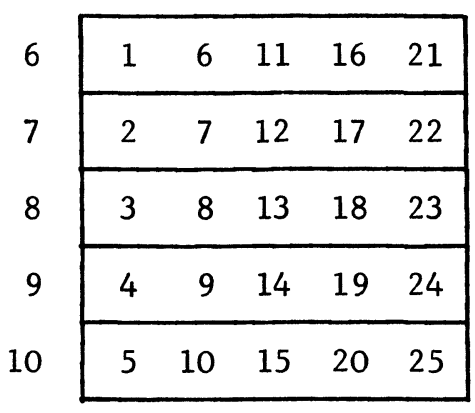

Rep. V

\begin{tabular}{l|rrrrr|}
21 & 1 & 17 & 8 & 24 & 15 \\
\cline { 2 - 6 } 22 & 11 & 2 & 18 & 9 & 25 \\
\cline { 2 - 5 } 23 & 21 & 12 & 3 & 19 & 10 \\
\cline { 2 - 5 } 24 & 6 & 22 & 13 & 4 & 20 \\
\cline { 2 - 5 } 25 & 16 & 7 & 23 & 14 & 5 \\
\hline
\end{tabular}

Rep. III

\begin{tabular}{l|rrrrr|}
\cline { 2 - 5 } 11 & 1 & 7 & 13 & 19 & 25 \\
\cline { 2 - 6 } 12 & 21 & 2 & 8 & 14 & 20 \\
\cline { 2 - 6 } 13 & 16 & 22 & 3 & 9 & 15 \\
\cline { 2 - 5 } 14 & 11 & 17 & 23 & 4 & 10 \\
\hline 15 & 6 & 12 & 18 & 24 & 5 \\
\hline
\end{tabular}

Rep. VI

\begin{tabular}{l|rrrrr|}
\cline { 2 - 5 } 26 & 1 & 22 & 18 & 14 & 10 \\
\cline { 2 - 5 } 27 & 6 & 2 & 23 & 19 & 15 \\
\cline { 2 - 5 } 28 & 11 & 7 & 3 & 24 & 20 \\
\cline { 2 - 5 } 30 & 16 & 12 & 8 & 4 & 25 \\
\cline { 2 - 5 } & 21 & 17 & 13 & 9 & 5 \\
\hline
\end{tabular}

Table (e) 30 treatments: $5 \times 6$ rectangular 1attice

Rep. I

\begin{tabular}{|c|c|c|c|c|}
\hline 1 & 2 & 3 & 4 & 5 \\
\hline 6 & 7 & 8 & 9 & 10 \\
\hline 11 & 12 & 13 & 14 & 15 \\
\hline 16 & 17 & 18 & 19 & 20 \\
\hline 21 & 22 & 23 & 24 & 25 \\
\hline 26 & 27 & 28 & 29 & 30 \\
\hline
\end{tabular}

Rep. II

\begin{tabular}{|c|c|c|c|c|}
\hline 1 & 12 & 17 & 22 & 27 \\
\hline 2 & 7 & 18 & 23 & 28 \\
\hline 3 & 8 & 13 & 24 & 29 \\
\hline 4 & 9 & 14 & 19 & 30 \\
\hline 5 & 10 & 15 & 20 & 25 \\
\hline 6 & 11 & 16 & 21 & 26 \\
\hline
\end{tabular}

Rep. III

\begin{tabular}{l|lllll|}
\cline { 2 - 5 } 13 & 1 & 7 & 13 & 19 & 25 \\
\cline { 2 - 5 } 15 & 2 & 8 & 14 & 20 & 26 \\
\cline { 2 - 5 } 16 & 3 & 9 & 15 & 21 & 27 \\
\cline { 2 - 5 } 17 & 10 & 16 & 22 & 28 \\
\hline 18 & 11 & 17 & 23 & 29 \\
\hline & 6 & 12 & 18 & 24 & 30 \\
\hline
\end{tabular}


Table (f). 36 treatments: $6 \times 6$ square lattice

\begin{tabular}{l|rrrrrr|}
\multicolumn{7}{c}{ Rep. I } \\
\cline { 2 - 7 } 1 & 1 & 2 & 3 & 4 & 5 & 6 \\
\hline 3 & 7 & 8 & 9 & 10 & 11 & 12 \\
\hline 4 & 13 & 14 & 15 & 16 & 17 & 18 \\
\hline 5 & 19 & 20 & 21 & 22 & 23 & 24 \\
\hline 5 & 25 & 26 & 27 & 28 & 29 & 30 \\
\hline & 31 & 32 & 33 & 34 & 35 & 36 \\
\hline
\end{tabular}

\begin{tabular}{c|cccccc|}
\multicolumn{1}{c}{ Rep. II } \\
\cline { 2 - 6 } 7 & 1 & 7 & 13 & 19 & 25 & 31 \\
\cline { 2 - 6 } 8 & 2 & 8 & 14 & 20 & 26 & 32 \\
\cline { 2 - 6 } 1 & 3 & 9 & 15 & 21 & 27 & 33 \\
\cline { 2 - 6 } 11 & 4 & 10 & 16 & 22 & 28 & 34 \\
\cline { 2 - 6 } 11 & 5 & 11 & 17 & 23 & 29 & 35 \\
\cline { 2 - 6 } 12 & 6 & 12 & 18 & 24 & 30 & 36 \\
\cline { 2 - 6 } & & & & &
\end{tabular}

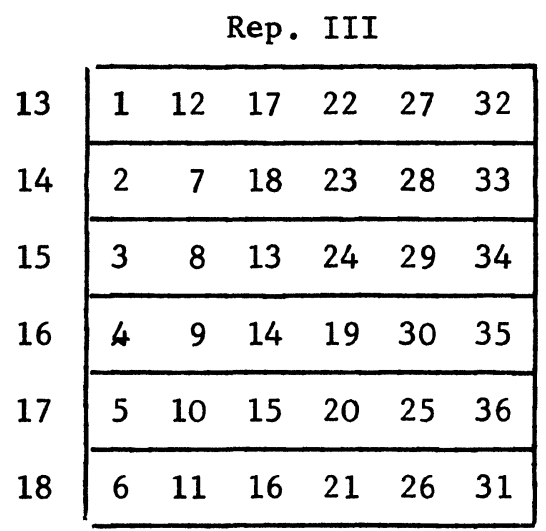

Note that these designs are unrandomized. Before use they should be randomized as detailed in Chapter 4 paragraph 4.221. 
Appendix 6. Determination of sample size

In order to ensure an adequate sample but at the same time to avoid wasted effort, the size of sample should be linked to the variability of the characteristic to be measured. This may be done as follows:-

1. Obtain an estimate of the population standard deviation (S) from previous work, a preliminary sample, or by crude estimation from

$$
\begin{aligned}
S=\frac{R}{4} & \text { where } R=\begin{array}{l}
\text { estimated range from the smallest } \\
\text { to the largest value likely to be } \\
\text { encountered in sampling. }
\end{array}
\end{aligned}
$$

The variation is usually expressed as the percentage coefficient of variation

$$
\begin{array}{ll}
\mathrm{CV}=\frac{100 \mathrm{~S}}{\text { mean }} & \mathrm{N} . \mathrm{B} . \quad \text { The mean is that of the } \\
\text { sample, and may have to be estimated if } \\
\text { no measurements are available. }
\end{array}
$$

2. The desired precision of the mean value must be stated, and is best expressed as $\pm E \%$ at a given level of probability.

3. The sample size $n$ may be determined from

$$
\mathrm{n}=\frac{(\mathrm{t} C \mathrm{CV})^{2}}{(\mathrm{E})^{2}}
$$

Where $t=$ value of $S$ tudent's $t$ for $n$ degrees of freedom and for given level of probability. Because the value of $t$ depends on the value of $n$, the above formula must be solved by trial and error. However, once $n$ becomes greater than $50, t$ may be assumed to have a constant value of 2 at the $95 \%$ level.

$$
\begin{aligned}
\text { e.g. let } C V & =50 \% \\
E & =10 \%
\end{aligned}
$$

assume $\mathrm{n}$ will be around $60 \therefore \mathrm{t}=2$

$$
\mathrm{n}=\frac{(2 \times 50)^{2}}{(10)^{2}}=100
$$

$t$ for $n=100$ is 1.99 instead of the assumed 2, but this will make very little difference, so take $n$ as 100 .

If the sampling fraction $f$ (i.e. the sample size over the total population) is not negligible, i.e. greater than .05 , then $n$ must be reduced according to

$$
\mathrm{n}=\frac{\mathrm{n}}{1+\mathrm{f}}
$$


Appendix 7. Nursery assessment

\section{Appendix 7.1 Nursery sampling technique}

Random sampling for nursery measurements and assessments can easily be done if the plants are in containers or are arranged in rows and columns with a consistent number of plants in each. Sampling may be on the basis of rows or of individual plants each of which can be assigned a number in relation to its position in the column and row.

\section{A. Sampling grid}

In seed beds and in transplant beds without regular spacing, random sampling is greatly facilitated by the use of a wire sampling grid. This should take the form of a rigid but light metal frame of side equal to the width of a standard bed. Equally spaced pairs of wires are used to provide sampling transects across the width of the bed. One wire is run across the frame at right angles to the transects, splitting each into halves. The transect width is conveniently $5 \mathrm{~cm}$ in a 1 metre frame or 2 inches in a 36 inch frame.

A sketch of a 1 metre square grid is given below, together with a table showing the sampling percent achieved by use of various numbers of transects.

\section{Sampling grid}

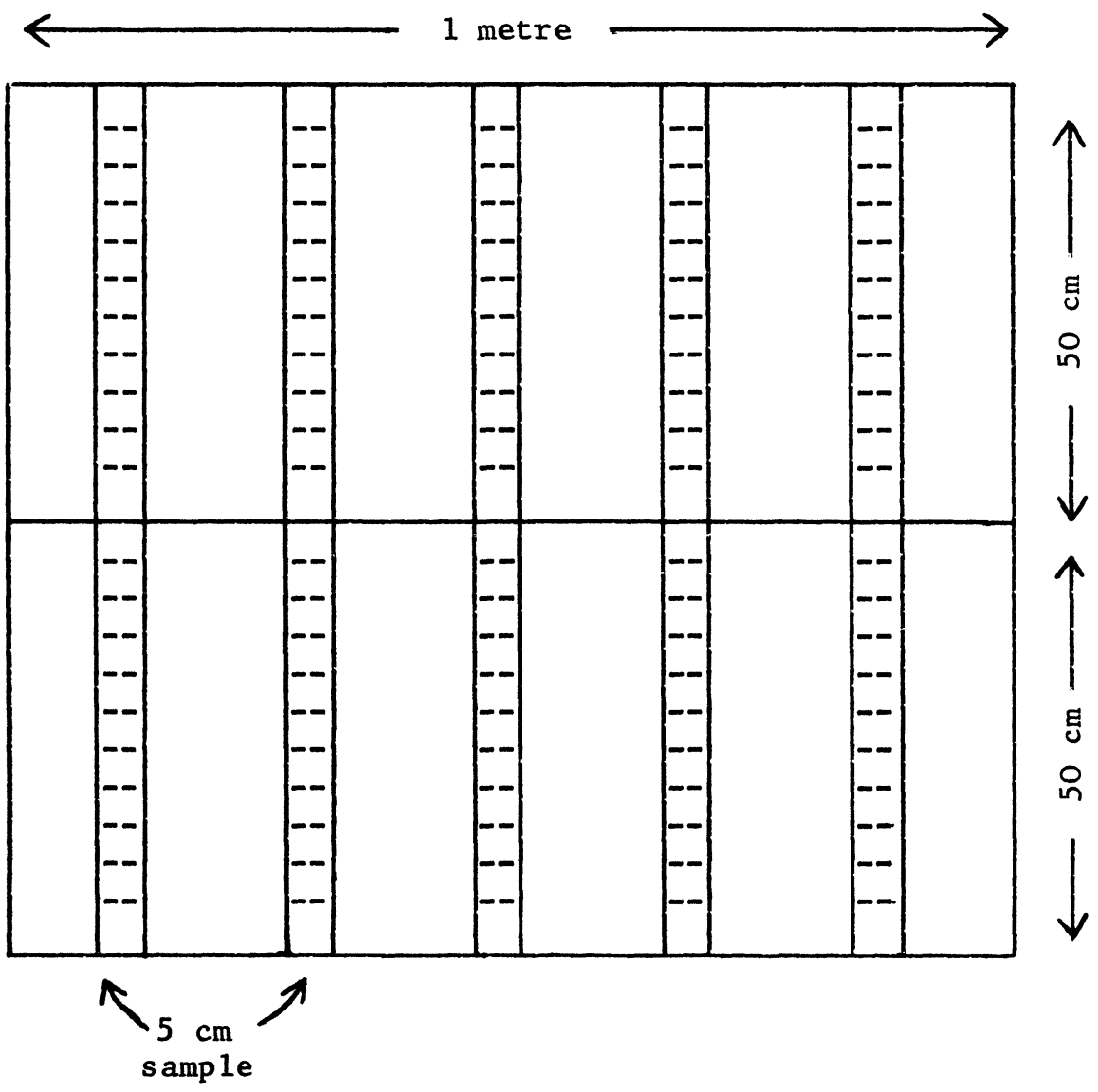


Appendix 7 (Cont.)

No. of grids

1

2

3

4

5
Sample size

$\begin{array}{llr}1 / 20 & \text { or } & 5 \% \\ 1 / 10 & \text { or } 10 \% \\ 1 / 6.6 & \text { or } 15 \% \\ 1 / 5 & \text { or } 20 \% \\ 1 / 4 & \text { or } 25 \%\end{array}$

\section{B. Sampling by area}

If sampling is to be on the basis of a percentage of area of seedbed $(5,10,15,20$ or $25 \%)$, a11 that is necessary is to assess the plants in the number of random transects which give the sampling percentage required.

For example, a $20 \%$ sample is obtained with 4 random transects - see table above. In the case of a $5 \%$ sample, requiring the use of one random transect, the measurement or counts are to be made in two randomly selected half-transects, one on either side of the central dividing line. Whole transects should be used when two or more are called for. 
Appendix 7.2. Characters of use in the assessment of species and provenance trials at the nursery stage. (The suggested sample sizes are based largely on experience with pines and could be modified for other genera especially hardwoods).

$1 . * 1,000$ seed weight. One sample per plot.

2. Seed length. 50 seeds per plot.

3. Seed width. 50 seeds per plot.

4. Seed coat thickness. May be found to correlate with precipitation evaporation ration of natural habitat. 50 seeds per plot.

5.* Germination percentage. Number of germinants expressed as a percentage of seeds sown in each plot.

6.* Rate of germination. Expresses vigour and may be indicative of heterosis. Requires count of germination daily for one month in each plot.

7. Number of cotyledons per seedling. Classify 20 seedlings per plot. (For species other than Dicotyledons)

8. Length of cotyledons. Measure second longest on each of the seedlings classified. (Width and shape may be important in hardwoods).

9. Cotyledon colour. Use plant tissue cards (Munse11 colour charts). Watch particularly for albinos which may die very early. Classify 10 seedlings per plot.

10. Hypocotyl length. Measure 25 seedlings per plot from ground level to top of hypocotyl.

11. Transplant dimensions. Sample 25 transplants per plot for height, diameter and also number of roots and length of longest root. Subsample 5 for dry and fresh weight.

12.* Pre-planting dimensions. Measure height and diameters on 25 plants per plot.

13. Survival in nursery. May be indicative of resistance to disease. Express number produced as percentage of number of seeds sown and number of seeds germinated in each plot.

14. Phenology. Season of flushing, bud formation, dormancy, leaf or branch production.

15. Damage. Biotic/climatic damage (rather than mechanical damage due to poor nursery technique) may be a discriminating characteristic.

* Characters suggested for priority assessment. 
Appendix 8. A selection of record forms

The suggested record forms included here are not the most elaborate that can be devised, but should be regarded as a minimum for adequate record keeping, with flexibility.

8.1A Nursery record form

8.1B Nursery assessment form

8.2 Subsequent history sheet

8.3A Periodic assessment form

8.3B Stem analysis form

8.3C Tree volume form 
- 202 -

8.1A Nursery record form

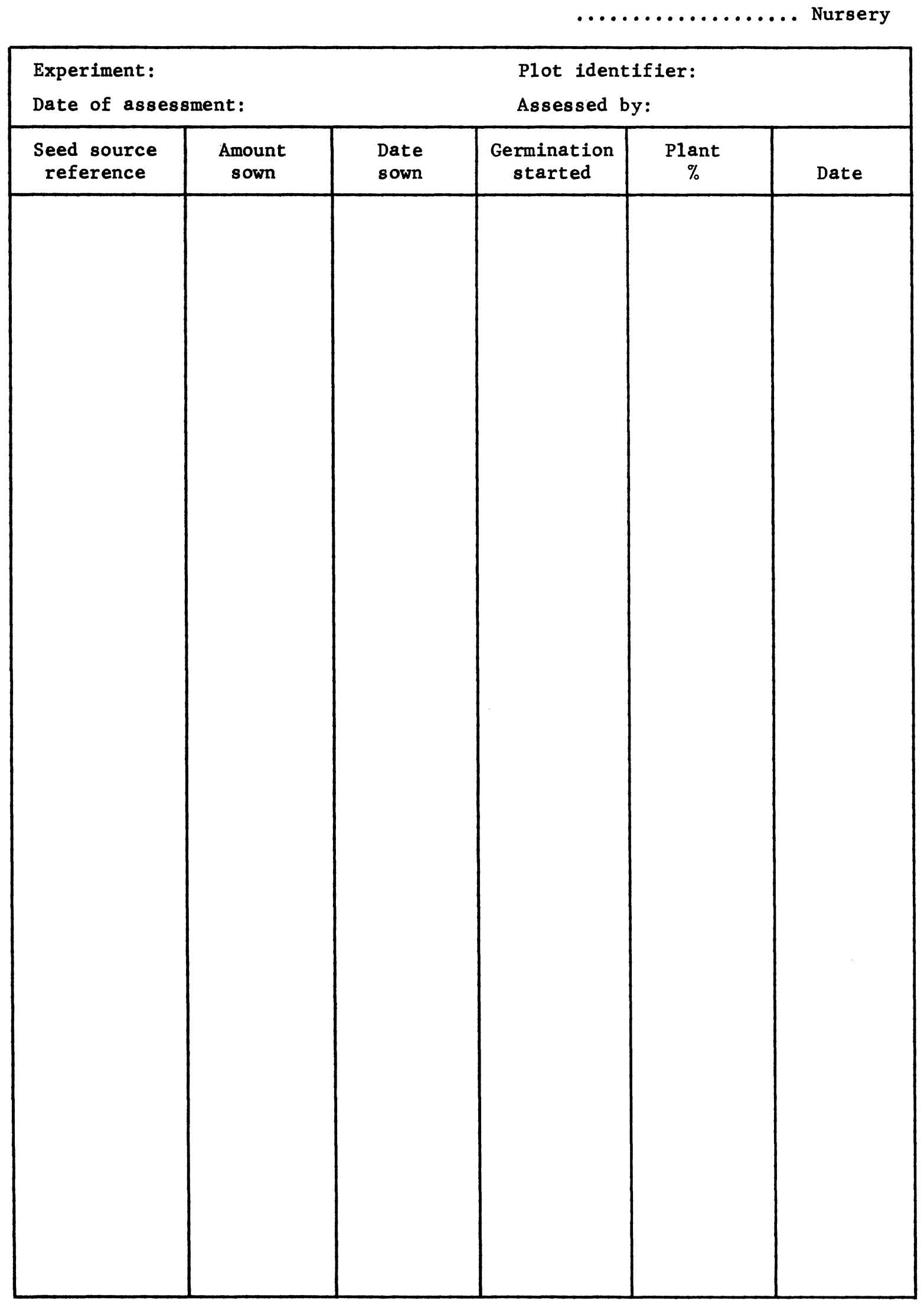


$-203-$

8.1B Nursery assessment form

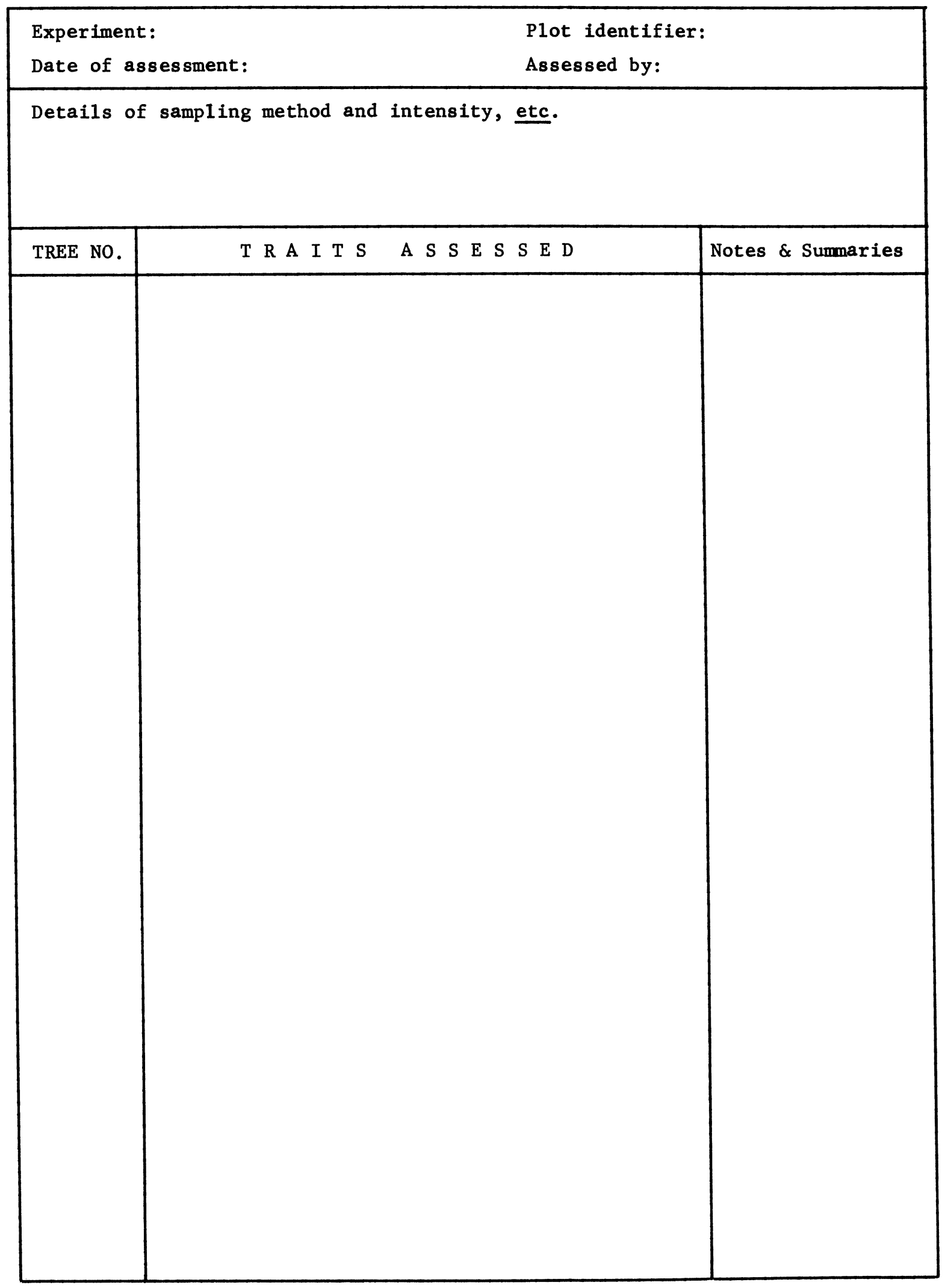


$-204-$

8.2 Subsequent history sheet

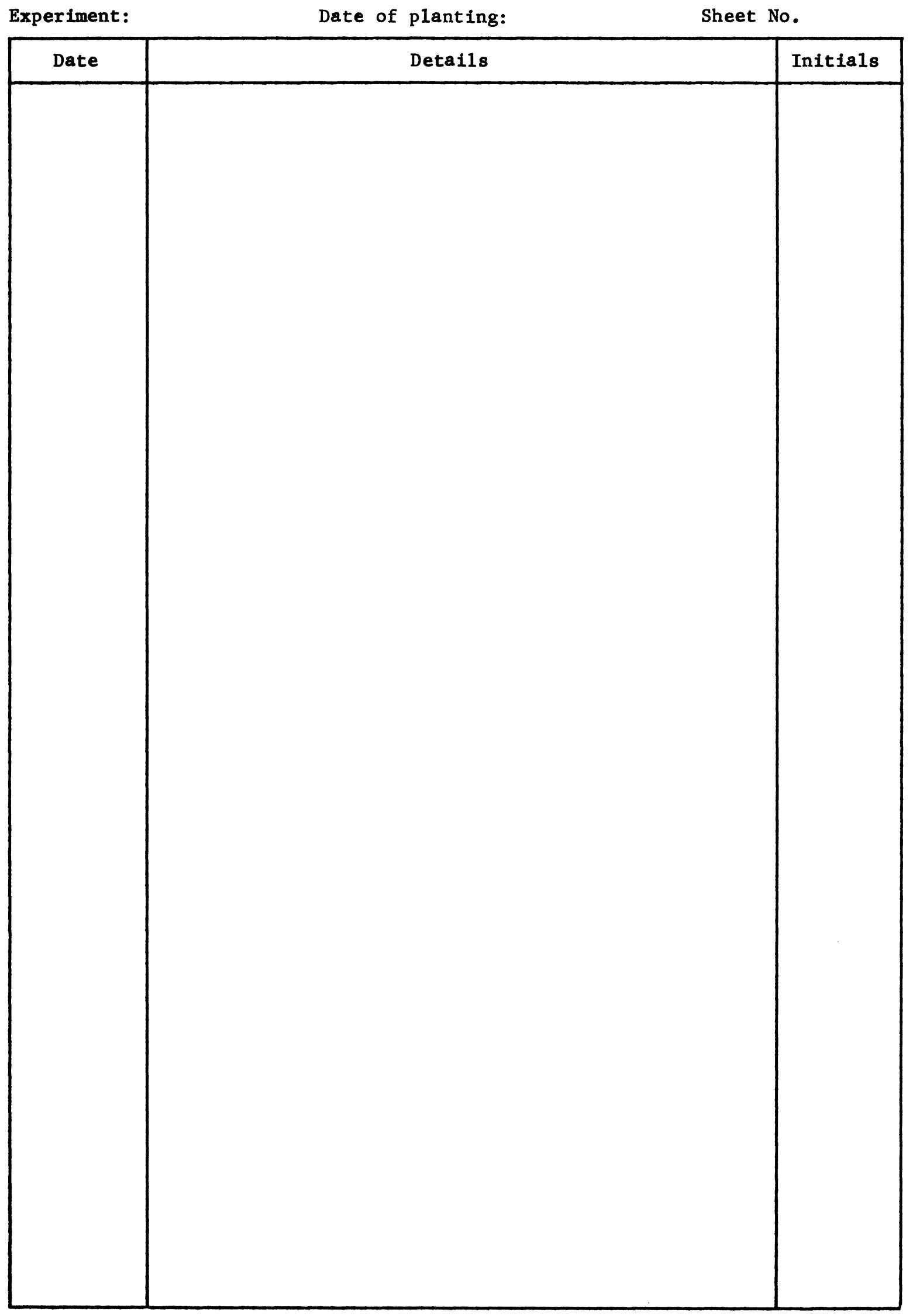




\subsection{A Periodic assessment form}

Experiment:

Date of assessment:
Plot identifier:

Assessed by:

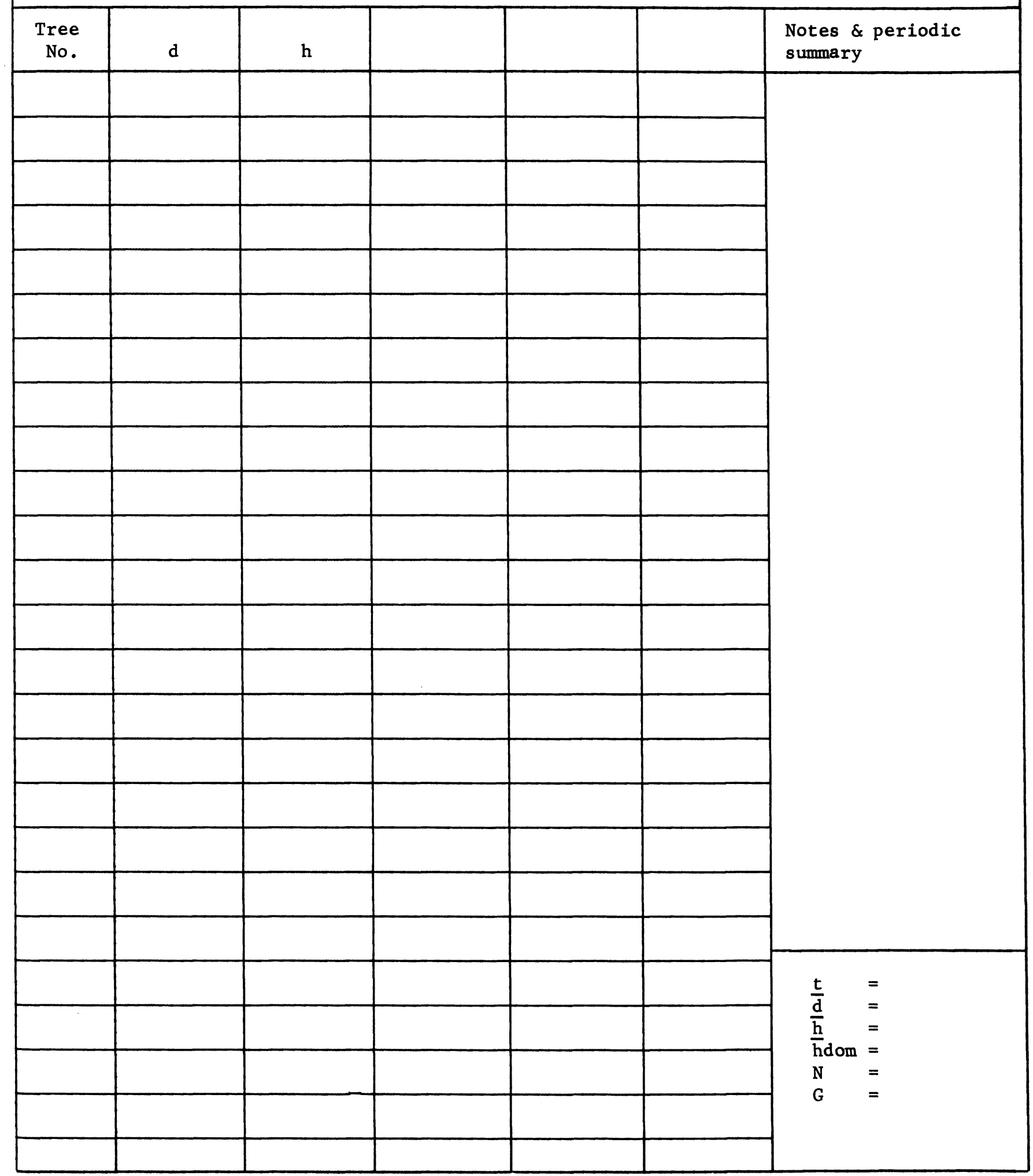




\subsection{B Stem analysis form}

Experiment:

Date of assessment:
Plot identifier:

Assessed by:

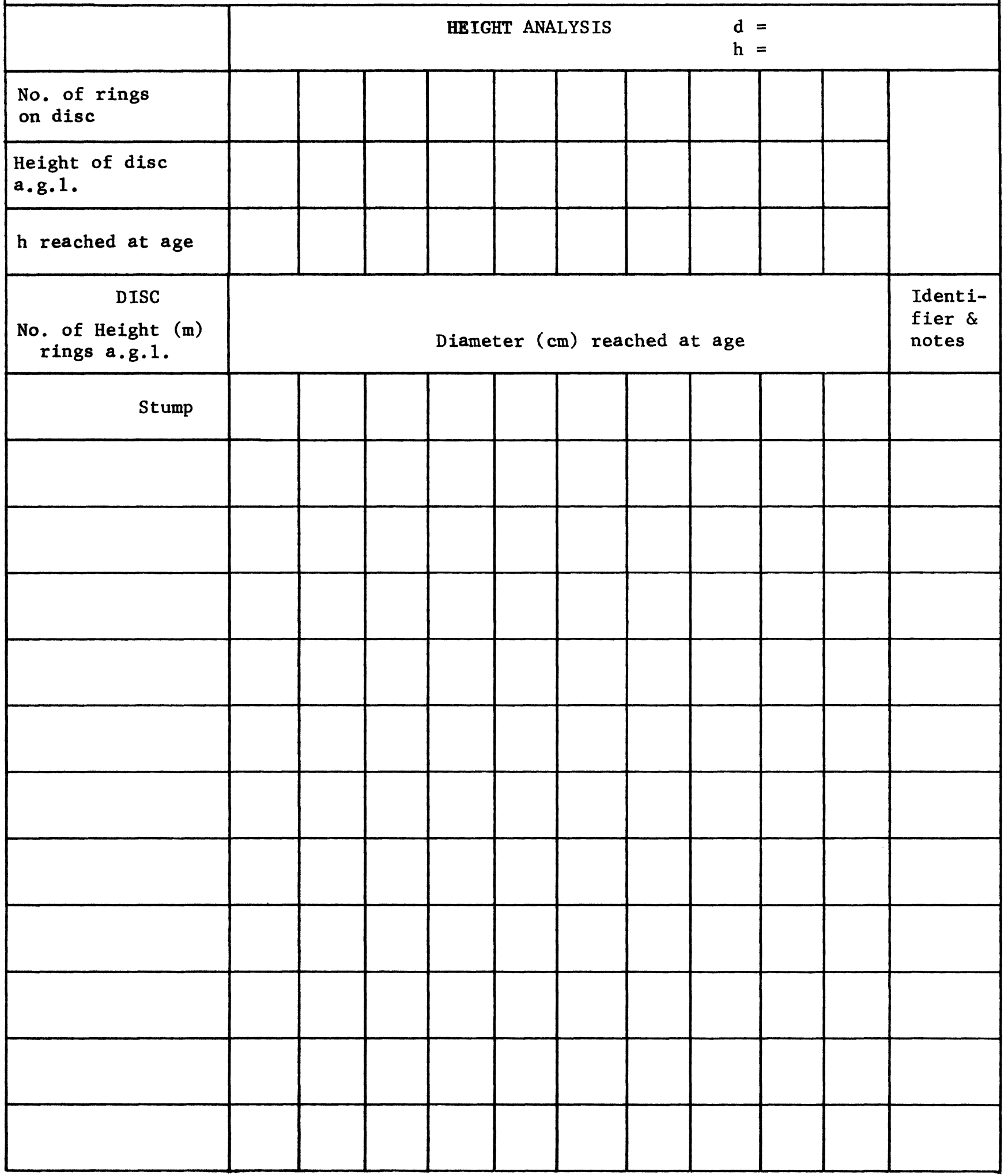




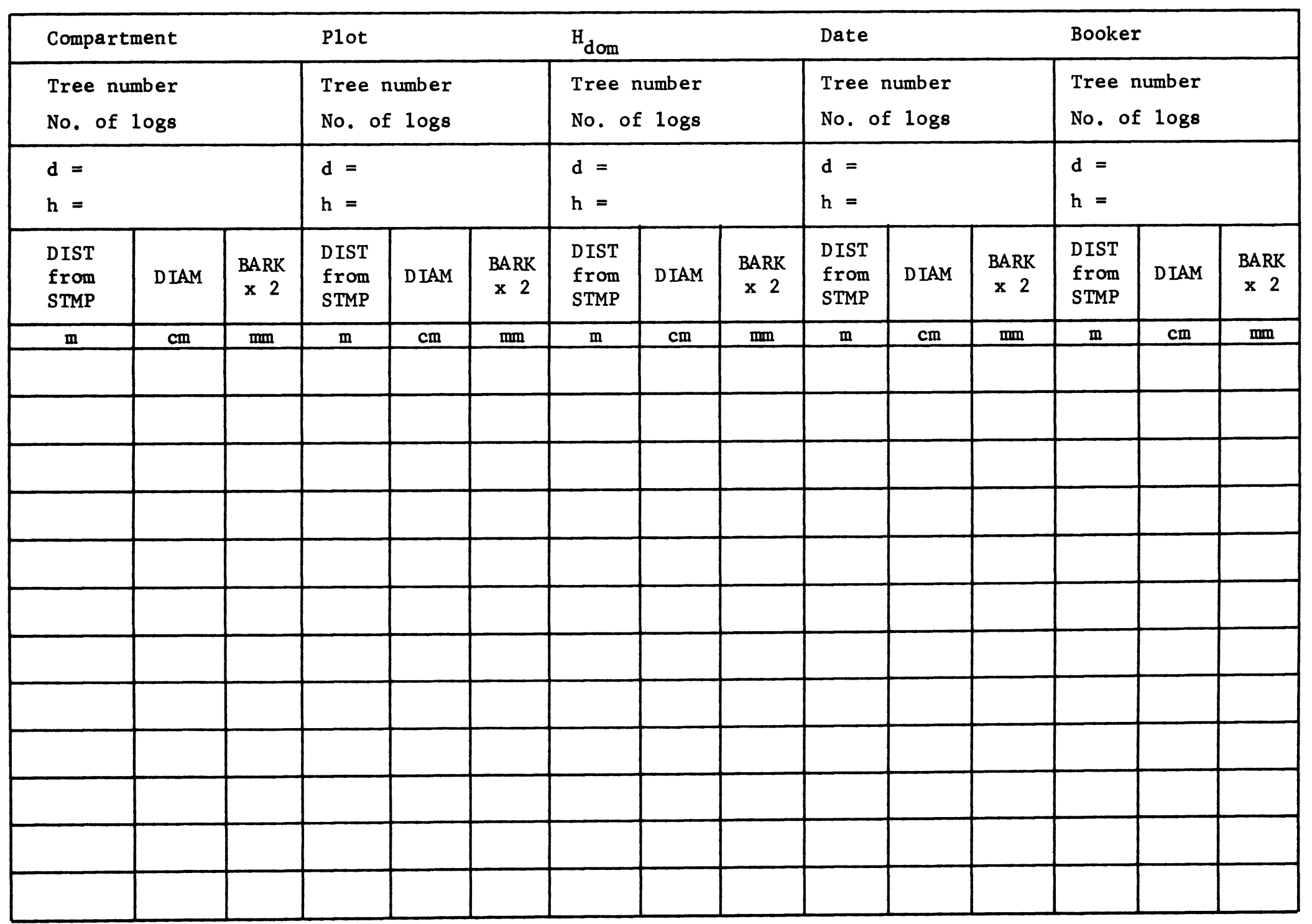


Appendix 9. A selection of analytical formats modified from Dawkins (1968)

9.1 Analysis of variance: single classification (fully random)

9.2 Calculation of contrasts and their confidence limits by Scheffé's method

9.3 Analysis of variance: simple randomised block

9.4 Analysis of variance: latin square (up to $6 \times 6$ ) 


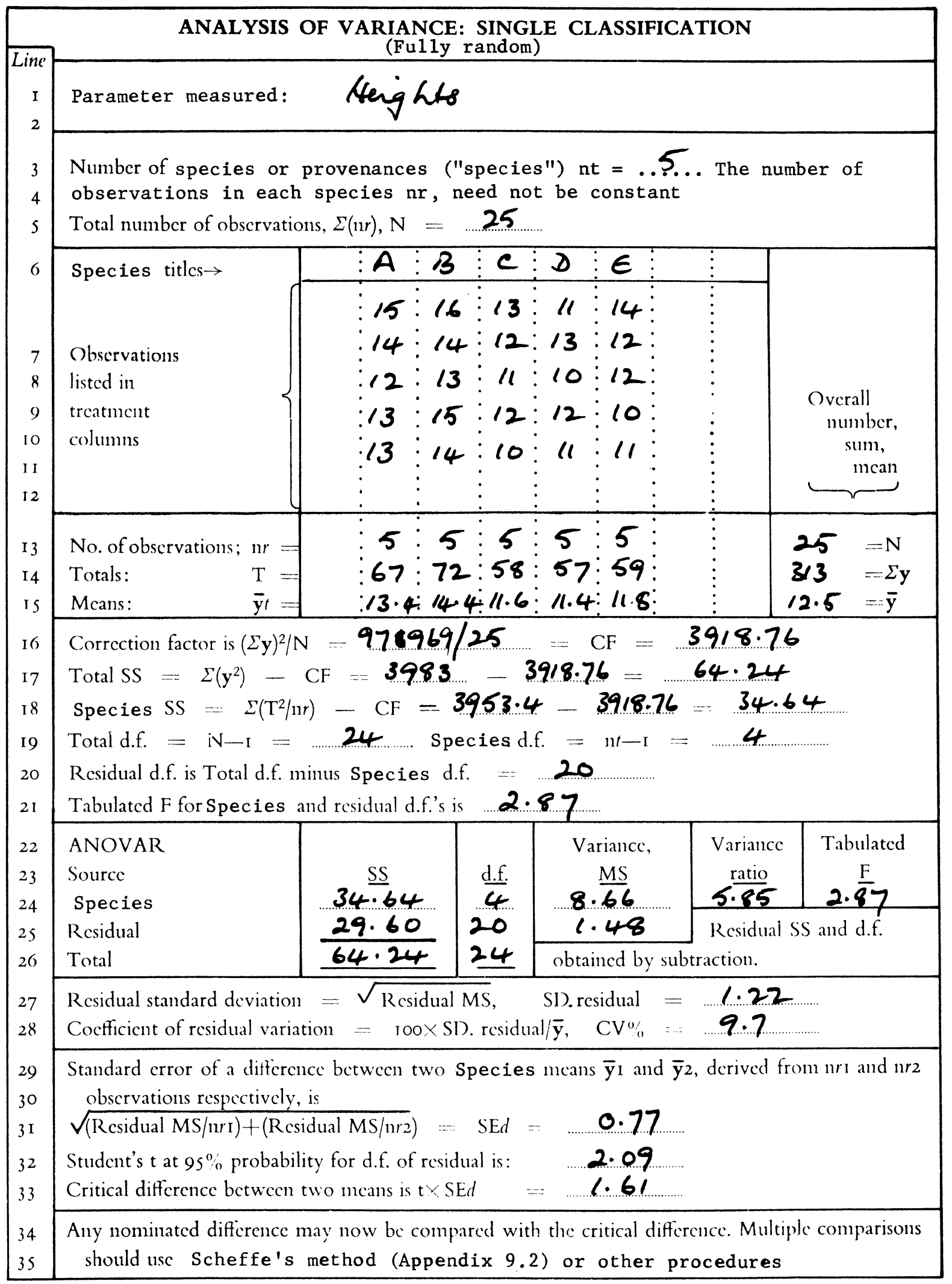


Appendix 9.2

$-210-$

CALCULATION OF CONTRASTS AND THEIR CONFIDENCE LIMITS BY SCHEFFE'S

Line METHOD

Obscrvations, $y$, are of: heights of five species $A, B, C, D, E$

From ANOVAR (from Statform: Appendix 9.2)

\begin{tabular}{|c|c|c|c|c|c|}
\hline Species & $34-\frac{S S}{64}$ & $\frac{\text { d.f. }}{4}$ & $8 \frac{M S}{66}$ & $\frac{\text { V-ratio }}{5.85}$ & $\frac{\text { Table F }}{2.87}$ \\
\hline Residual: & 29.60 & 20 & 1.48 & & \\
\hline
\end{tabular}

Species

$\mathrm{n} r=$ number of replicates of each

Titles | Totals

\begin{tabular}{c|c|c} 
& Totals & \\
$\mathrm{T}$ & $\mathrm{n} r$
\end{tabular}

A

$B$ $\mathrm{T}$

A $\quad 67$

$>$

$E$ 67

72

58

57

59

$\times$ Residual MS,

$\times$ Species d.f.

$\times$ Table F,

Square-rooted,

If $S$ is greater than $|C|$, then confidence limits will span zero and the contrast is not significant at the probability level selected for table F.

Confidence limits $\left\{\begin{array}{l}\text { Upper, } \mathrm{C}+\mathrm{S}= \\ \text { Contrast, } \mathrm{C}= \\ \text { Lower, } \mathrm{C}-\mathrm{S}=\end{array}\right.$

sig.

$\div .74:$

$-.2 \cdot 42:$

$-4 \cdot 10$ aig.

:4.96:

2.70 :

$: .44$
A: $\lambda$ 


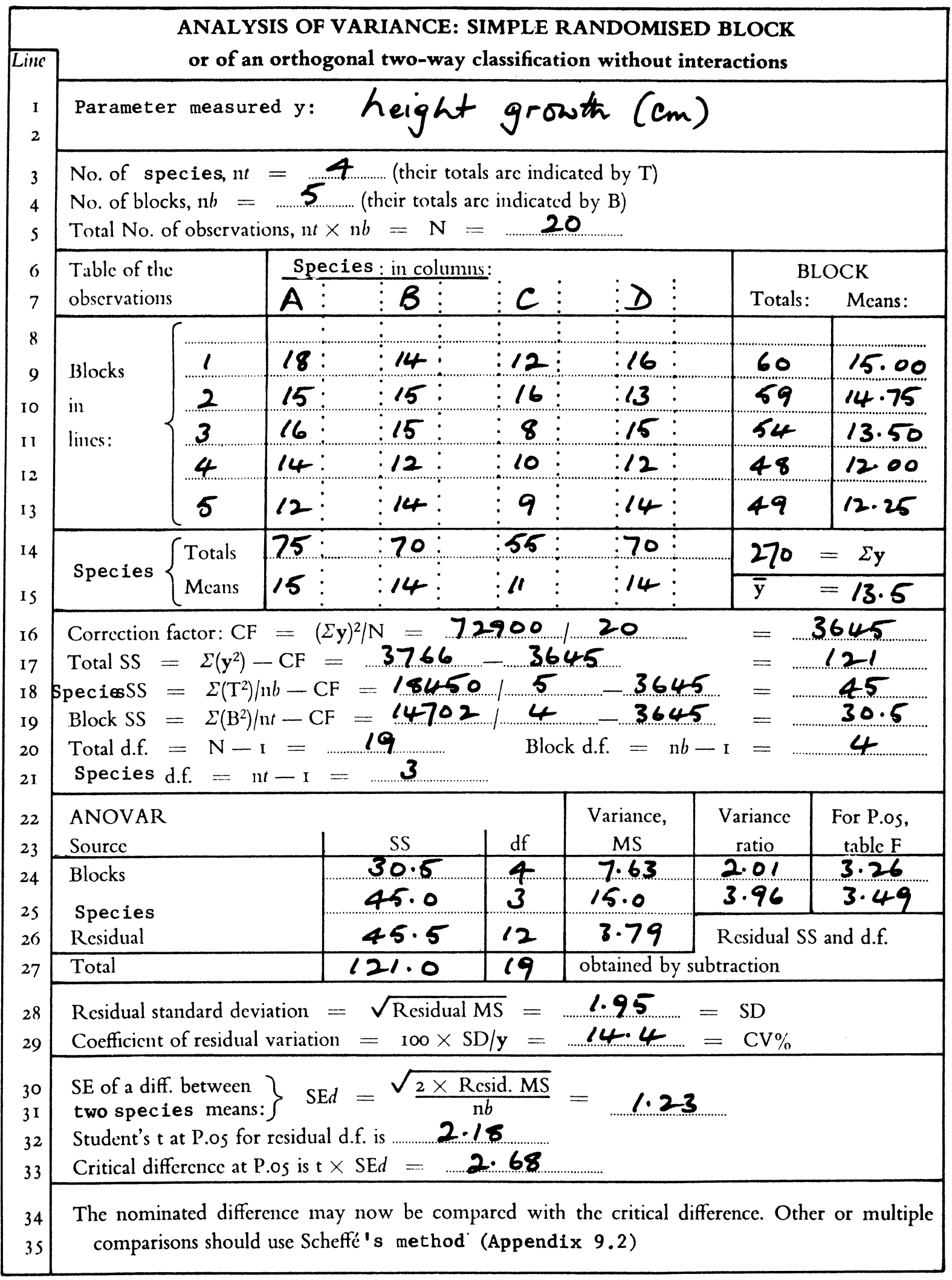




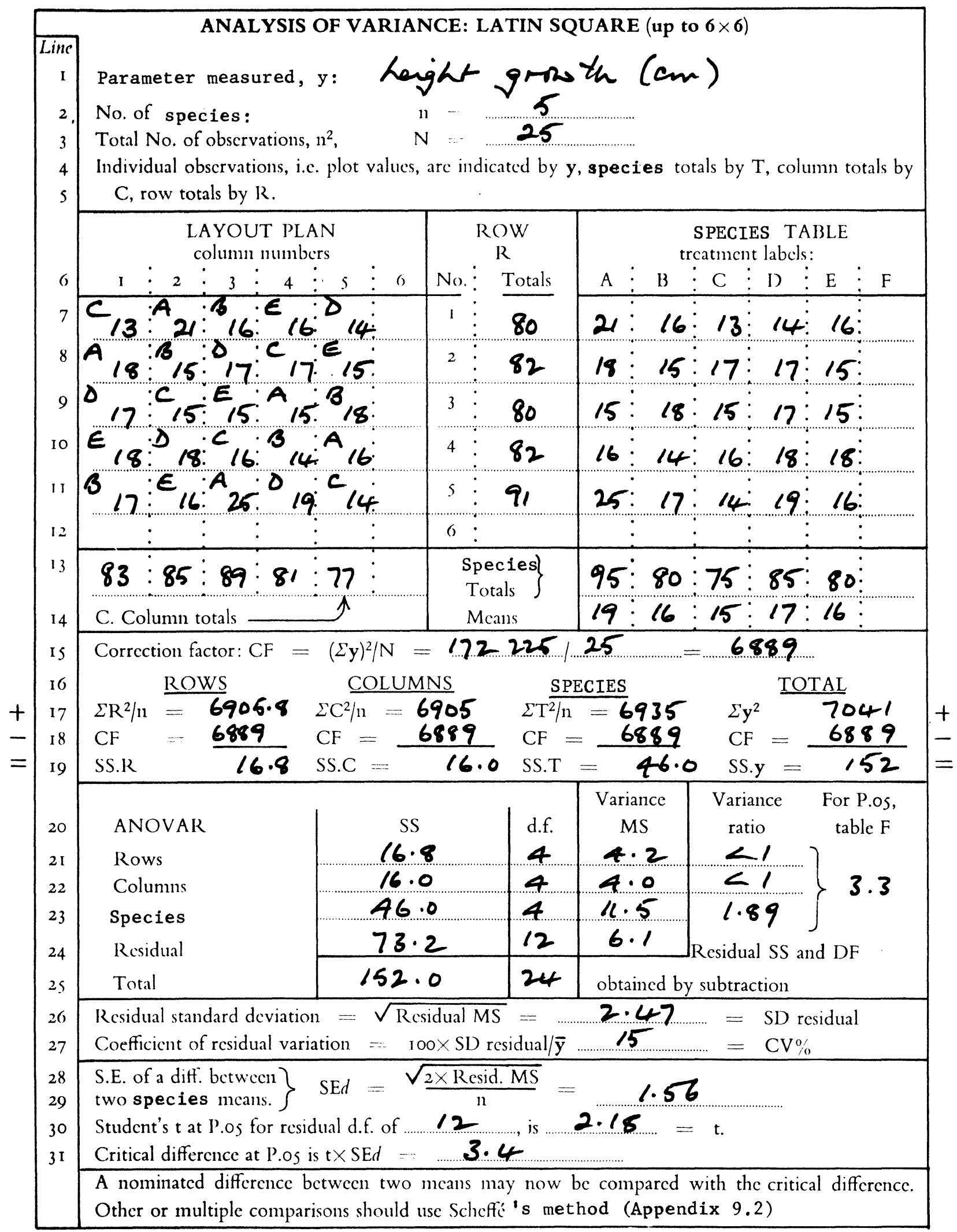


Appendix 10. Sample plot procedure in commercial plantations

Objects of permanent growth or sample plots

A network of permanent sample plots (P.S.P.s) in a continuous forest inventory (C.F.I.) correctly laid out and systematically maintained and assessed provides the forest manager with the most reliable source of information known on:-

(1) how forest stands are changing with time,

(2) how stands vary with site and treatment,

(3) the relations between stand parameters (e.g. stand height, basal area, commercial volume, etc.) and of individual tree parameters (height, diameter, form, wood quality and value, etc.) with stand density.

Continuous forest inventory provides not only the data essential for studies of forest yield leading to the construction of yield models and tables but also the "monitoring system" of the forest.

With a well run C.F.I. the forest manager can detect departures in the growth behaviour of his stands from the expected norm which otherwise may go unnoticed until serious economic consequences are felt or, at worst, until final felling reveals unexpected deviations from estimated yields or quality. The objects of C.F.I. are not to be confused with those of silvicultural experiments, or with growth stock inventories using temporary sample plots. Subjectively sited and carefully designed experimental plots are essential for studying the effects of specific treatments on stand and tree parameters. Results of such research are needed for the proper interpretation of C.F.I. data. Formal experiments and a network of randomly located growth plots are complementary methods for understanding the behaviour and performance of the forest and not alternatives. A temporary sample plot survey is an inventory tool and unless analysed in conjunction with increment borings or stem analyses has a very different purpose from that of growth plots. When applying the results of an analysis of C.F.I. data which will be provided, say, in the form of a yield table or a mathematic growth model, it will be necessary to assess the quality class of a compartment or part of a compartment. A survey using some form of temporary sample plot inventory will then be necessary in order to use the appropriate yield table or growth model for a particular stand. Provided the information from P.S.P.S is sufficient and accurate the type of temporary inventory required for making growth and yield predictions can be very simple and cheap. Where up-to-date aerial photographs are available these may provide the necessary data without additional field work.

\section{Size and numbers of P.S.P.s required}

As a rough guide 50 plots 0.04 ha ( 0.1 acre) in area per stratum (i.e. climatic or topographic unit) regularly distributed throughout all age classes might be suitable for an even-aged plantation species grown on a rotation of 30 or 40 years. With very variable site conditions more plots may be necessary, but less if site conditions are uniform. In 
practice a balance between the ideal number of plots needed to cover varying conditions of site and treatments and the resources available for maintenance and reassessment has to be found. It it more important that the absolute number of plots exceeds a certain minimum (in most cases 30 per stratum/species) rather than that a certain percentage sample is attained.

Strata may be subdivided into secondary units (e.g. site types or administrative units, such as compartments) within which two or more plots may be located. Two-stage sampling of this type will provide statistically valid data for assessing the variability of growth within the secondary units and will ensure that the plots are distributed in an efficient manner throughout the plantations.

\section{Location of plots}

Plots must be randomly located in the office on a 1:10,000 or similar scale map, using a system of coordinates. The plot locations so marked must be strictly positioned in the field as shown on the map.

Where sampling is to be in two stages procede as follows:-

a) use random coordinates to select the secondary units in which plots will be located

b) use two or more pairs of random coordinates to determine the position of the plots within the selected secondary unit.

A plot position should however be rejected if:-

a) it falls within a distance equal to a plot diameter of a stratum or secondary unit boundary (but not if it falls in a gap within the stand)

b) the plot area includes more than one site type (this could only occur in a single stage sampling plan).

c) it fallswithin some predefined distance (say $25 \mathrm{~m}$ ) from a preexisting plot.

In any of the above cases a new plot location selected with a fresh pair of random numbers will be chosen. In no event will the plot merely be

"shifted" subjectively when being sited.

Layout of plots

Two conflicting needs have to be reconciled when-laying out and marking a P.S.P. on the ground:-

(1) the need to mark the position of the plot clearly on the ground and to identify each tree individual 80 that the plot may be refound and every tree identified after a period of up to 4 years

(2) the need to lay out the plot in such a way as to ensure that it receives the same treatment as the surrounding stand 80 that it remains representative of the stand in which it lies. 
It is recommended that: the plot centre be marked with a stout centre stake or concrete pillar and trenches $0.5 \mathrm{~m}$ deep aligned $\mathrm{N}-\mathrm{S}$ and E-W.

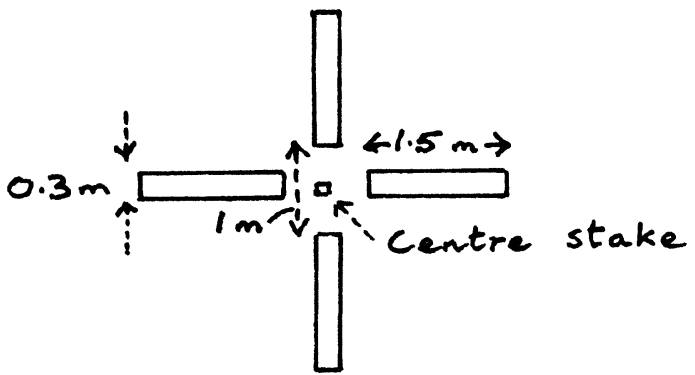

The trenches should not meet in the centre so that an observer may stand directly over the centre on firm ground, if necessary.

Each tree must be marked with an aluminium nail and aluminium or plastic tag with the tree number stamped or painted on it positioned at exactly $1 \mathrm{~m} 80 \mathrm{~cm}$ on the uphill side of the tree, that is $50 \mathrm{~cm}$ above breast height. On no account should nails and tags be inserted at breast height. In areas where nails may be removed by passers-by all tree numbers should be painted in durable paint on the side of the tree facing the plot centre and a ring painted at breast height $(1.3 \mathrm{~m})$. In such cases need ( 1 ) above demands conspicuous marking at the expense of need (2). Therefore clear instructions to the management staff must ensure that plots so marked are treated in the same way as the rest of the stand.

The plot number must be marked with a stamped aluminium tag

a) on the plot centre post

b) on the tree nearest the centre at ground level

c) on a small notice board at the nearest roadside or ccess point

The trees must be numbered systematically so that even if all tree numbers have been removed or rendered indecipherable since the last assessment or inspection the plot can be renumbered without ambiguity. It is recommended that trees be numbered radially in a clockwise direction starting either at "north" or at the nearest tree to the centre. If two or more trees lie exactly on the same radius the tree nearest the centre is numbered first.

\section{$\underline{\text { Records }}$}

Four types of record must be carefully maintained:-

1. Record of plot location $\underline{\mathbf{e}} . \mathrm{g}$. on a 1:10,000 scale map

2. Record of site description

e.g. on Form A1

3. Record of tree positions

e.g. on Form A2

4. Record of periodic assessments of the standing crop 
5. Record of removals such as by thinning, death, theft, windthrow or final felling. In every case except theft detailed records of "felled trees" measurements should be kept. e.g. on Form $C$

\section{Plot location}

This should be marked accurately on a 1:10,000 or similar scale map. Attached to the map will be a schedule of all plot numbers marked on the map listing true bearings and horizontal distances from an identifiable mark at a convenient point of access for each plot. Two copies of each map should be made, one to be kept at the Forest Station and one at the Research or Inventory Headquarters Office.

\section{Site description}

Essential information required for classifying 'site' is listed on Form A1. Ideally these data should be recorded at time of establishment but it may be completed at any time before analysis.

Al1 the other entries should be simple to understand and record except rooting depth. Often this can be referred to as 'not limiting' but in other cases a soil pit must be dug and rooting observed.

The age of the crop when the observation is made must be noted.

\section{Tree positions}

A map of tree positions can be prepared from the data of bearings and distances of individual trees from the plot centre, as recorded on Form A2.

In younger stands only trees lying within the plot boundary will be recorded. In older crops where stem numbers have fallen below 30 per plot (or 750 per ha) competing neighbour trees standing immediately outside the plots should be numbered trees within the plot boundary. These additional trees will be numbered from any convenient number higher than the highest tree number within the plot. Diameters of neighbour trees will be measured at each assessment as if they were within the plot. The object of this record is the provision of measures of growing space for individual trees. It also enables re-numbering to take place if the numbers on the trees are lost.

\section{Periodic assessments of standing crop}

Essential information required here is indicated on Form B. A separate form for recording actual hypsometer measurements, distances and angle of slope should be used in the field. Heights calculated from the field data are then transferred to form $B$. Heights must be measured by two observers from opposite sides of a tree simultaneously. If an error of more than $5 \%$ is found between them the tree is remeasured. Only the average checked height will be recorded on the assessment form. The summary entries are optional and merely for use by local staff if desired. Additional information on the plot as a whole (disease rating, crown closure, etc.) can also be recorded if needed. 


\section{Remova1s}

These should be recorded on a separate form. 'Form $C$ ' is an example indicating the kind of information required. Detailed measurements for deriving total and merchantable volume both over and under bark are required at final felling in which case all the information called for in Form $C$ would be provided. Normally only diameter breast height and total height need be measured on trees removed as thinnings, windthrow, disease, etc. In such cases Form $C$ would be partially completed only. There are theoretical advantages in measuring felled trees at the mid-point of 5 logs each one-fifth the total length of the tree, rather than at the midpoint of $\log$ lengths fixed arbitarily at, say, $3 \mathrm{~m}$. Where the latter practice is customarily used it should be continued provided data for calculating total volume is included (i..e. total length is measured and diameters are taken to a reasonably small 'timber point' so that the volume of the top 'cone' can be calculated accurately). A different form would be needed in such cases. On Form C 'Diameter at $10 \%, 30 \% \ldots .$. ' etc. means the diameter measured at 10, 30\%, etc., of the total length measured from the top. Two lines are required for each tree, one for overbark and the second for underbark. The appropriate indication is made in the column $O B / U B$. 'Total length' should include stump height and would in fact be measured from a breast height or $1 \mathrm{~m} 80 \mathrm{~cm}$ point marked before felling. 'Reason for felling' could be indicated by simple abbreviations such as $T$ for thinning, $F$ for final felling, $D$ for dead, etc.

Form $C$ would be completed by the officer in charge of the station in which the plot lies whenever any accidental removal occurred from a plot. Thinnings and final fellings would be recorded in the same form by either research or management staff as directed by the officer in charge of the P.S.P. programme.

\section{Annua 1 maintenance}

Plots must be inspected at least once a year. Any damage or removals will be recorded and plot marks and tree numbers renewed if necessary.

\section{Assessments}

The interval between assessments will depend on the rate of growth of the species and the work load of the staff responsible. It should be such that removals from the stand can be detected quickly if the plot is not visited annually.

These must be carried out by responsible staff trained and tested in the necessary mensurational techniques. As indicated above two members of the assessment team must record heights independently and remeasurements carried out if the two estimates differ by more $5 \%$. As dominant height (height of the 100 trees of largest diameter per ha) is the most widely used index of site quality this must be assessed with the greatest care. For a plot 0.04 ha in area this means measuring only four trees. Gross inaccuracy in even one measurement can distort the site quality assessment. Therefore special care is needed in assessing dominant heights. Mean height, whenever this is required, should be measured on 10 trees selected systematically from the list of tree numbers, and may include trees already measured 
to assess dominant height. Heights should be measured to the nearest completed metre and diameters to the nearest $0.1 \mathrm{~cm}$. Metric measurements are standard in all scientific work and should of course be used in preference to imperial units.

\section{Maintenance of plots through more than one rotation}

Before final felling the plot centre must be marked in such a way as to ensure its relocation after felling and replanting. Continued observation of a plot through to second and subsequent rotations will be invaluable in yield studies and provide important evidence of fertility changes.

Maximum basal area control plots

There is evidence of the value of maximum basal area as an indication of site potential at a given age and as a standard by which to assess thinning intensity. The establishment of unthinned plots systematically sited in association with selected, or even all, P.S.P.s will provide such information. These control P.S.P.s should be sited on the same contour (í.e. catenary position) as its pair at say a distance of $100 \mathrm{~m}$. Circular plots of $25 \mathrm{~m}$ radius of ca. 0.2 ha area are recommended. This will give an assessment plot of 0.04 ha with radius $11.28 \mathrm{~m}$ plus an adequate surround ca. $13 \mathrm{~m}$ wide. It is desirable to establish these control plots at the same time as its neighbour plot is established before first thinning, but they will still be valuable if established later in older stands any time before the final thinning is due.

The management specifications for control plots are simple:-

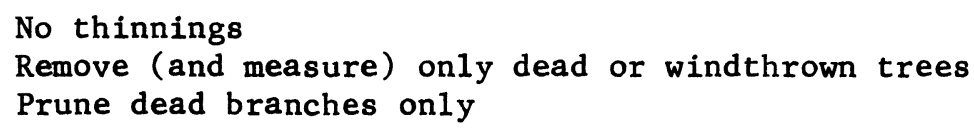

The boundaries of such plots must be conspicuously marked with distinctive coloured paint rings. Individual trees will be numbered in the inner assessment plot in exactly the same way as in a standard plot. 
$\underline{\text { FORM A1 }}$

EXAMPLE OF INFORMATION REQUIRED ON A PSP SITE DESCRIPTION FORM

\begin{tabular}{|c|c|c|c|c|c|c|c|c|c|c|c|c|c|}
\hline \multicolumn{14}{|l|}{ PLOT IDENT IF ICAT ION } \\
\hline \multicolumn{14}{|l|}{$\begin{array}{l}\text { SPECIES } \\
\text { PROVENANCE } \\
\text { SEED LOT NUMBER }\end{array}$} \\
\hline \multicolumn{14}{|l|}{$\begin{array}{l}\text { PLANT ING YEAR } \\
\text { METHOD OF ESTABLISHMENT }\end{array}$} \\
\hline \multicolumn{14}{|l|}{$\begin{array}{l}\text { AREA OF } \text { PLOT } \\
\text { AREA OF } \text { BLOCK/COMPARTMENT }\end{array}$} \\
\hline \multicolumn{14}{|l|}{$\begin{array}{l}\text { MAP REFERENCE } \\
\text { ACCESS POINT } \\
\text { DIRECT IONS FROM ACCESS } \\
\text { POINT }\end{array}$} \\
\hline \multirow{3}{*}{$\begin{array}{l}\text { CL IMATE } \\
\text { MEAN MONTHLY RAINFALL } \\
\text { MAX MONTHLY TEMPERATURE } \\
\text { MIN MONTHLY TEMPERATURE } \\
\text { OTHER INFORMATION } \\
\text { CLIMATIC ZONE } \\
\text { CL IMAT IC INDEX }\end{array}$} & $\mathrm{J}$ & $\bar{F}$ & $\bar{M}$ & $\bar{A}$ & $\bar{M}$ & $\mathrm{~J}$ & $\mathrm{~J}$ & A & $\mathrm{S}$ & 0 & $\mathrm{~N}$ & $\mathrm{D}$ & YEAR \\
\hline & & & & & & & & & & & & & \\
\hline & & & & & & & & & & & & & \\
\hline \multicolumn{14}{|l|}{$\begin{array}{l}\text { ALTITUDE } \\
\text { SLOPE IN DEGREES } \\
\text { NATURE OF SLOPE* } \\
\text { ASPECT } \\
\text { TOPOGRAPH IC POSITION* } \\
\text { EXPOSURE RATING }\end{array}$} \\
\hline \multicolumn{14}{|l|}{$\begin{array}{l}\text { SOIL TYPE } \\
\text { SOIL TEXTURE } \\
\text { ROOTING DEPTH }\end{array}$} \\
\hline \multicolumn{14}{|l|}{$\begin{array}{l}\text { ORIGINAL VEGETATION TYPE } \\
\text { IF KNOWN }\end{array}$} \\
\hline \multicolumn{14}{|l|}{ UNDERSTOREY VEGETATION } \\
\hline \multicolumn{14}{|l|}{ GROUND FLORA } \\
\hline $\begin{array}{l}\text { NOTE ANY PECULARITIES OF } \\
\text { THE SITE THAT COULD BE } \\
\text { INFLUENCING FOREST } \\
\text { GROWTH }\end{array}$ & & & & & & & & & & & & & \\
\hline
\end{tabular}

* See Notes overleaf 


\section{NOTES TO FORM A1}

CL IMATE :

NATURE OF SLOPE:

TOPOGRAPHIC POSITION:

EXPOSURE RATING:
This entry may be common to a group of plots. Indicate in detail on one form only and refer to this plot on the remaining plot forms in the group.

Indicate whether CONCAVE, LINEAR or CONVEX, etc.

Record on a scale of $1-5$ where 1 is ridge, 5 is valley bottom and 3 is mid-slope unaffected by ridge of valley influences. 2 and 4 indicate that ridge or valley effects are marginal respectively. Sketch position of plot in relation to the topography either using rough contour lines

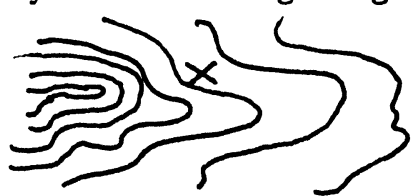

or drawing a profile as if seen in two directions:

(1) in the direction of ASPECT, and

(2) at right angles to the direction of aspect.

e..g. (1)

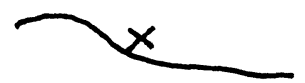

(2)

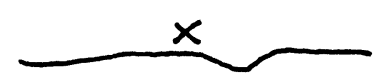

Record on a scale of 1-3 where

1 = exposed site; exposed to winds especially during the establishment phase.

2 = normal; average exposure.

3 = sheltered site. 
DATA REQUIRED FOR PLOTTING TREE POSITIONS

PLOT

DATA RECORDED

RECORDED BY

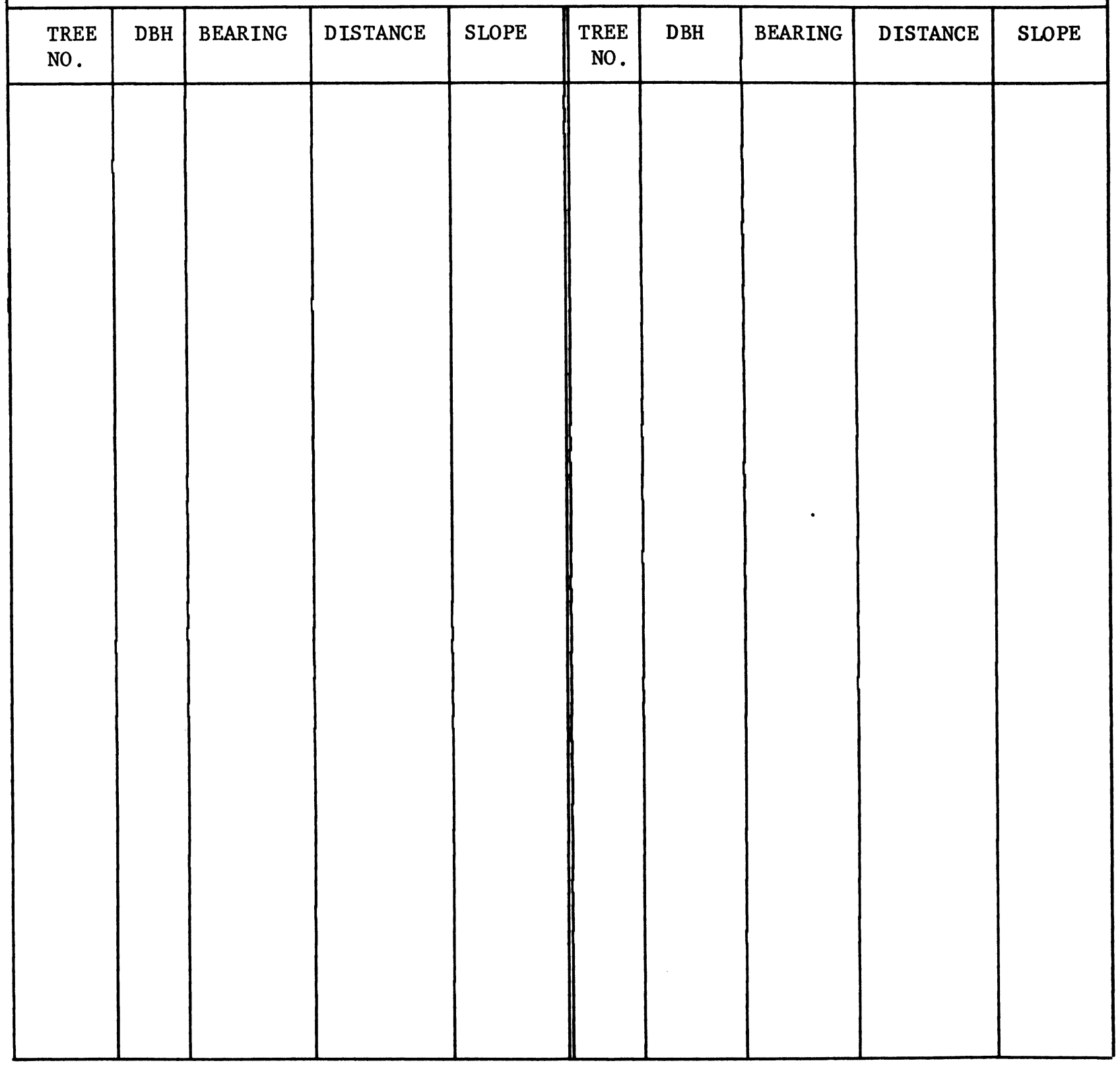

Note that all competing neighbours (i.e. all trees whose crowns are, or will be before next thinning, in contact with any tree within the plot) immediately outside the plots should be included if there are less than 30 living trees within the plot. 
FORM B

EXAMPLE OF THE INFORMATION REQUIRED ON THE PERIODIC ASSESSMENT FORM

MEASUREMENTS ON STANDING TREES

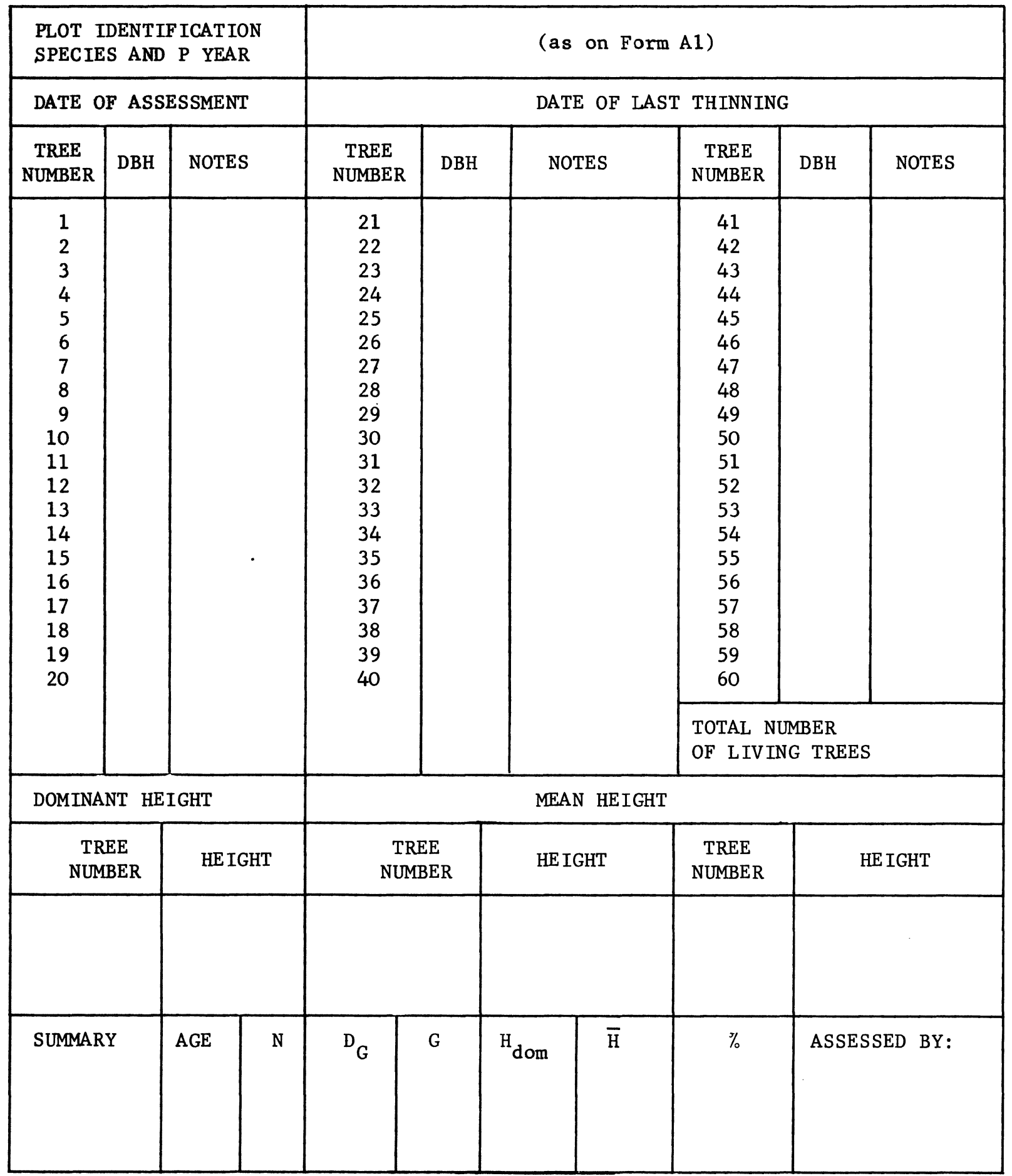

$N=$ stems per ha

$\mathrm{D}_{\mathrm{G}}=$ diameter of mean-basal-area tree or quadratic mean diameter

$\underline{H}^{G}=$ dominant height (‥e. mean height of 100 trees of largest diameter per ha)

$\overrightarrow{\mathrm{H}}^{\mathrm{dom}}=$ mean height (․․… arithmetic mean of all, or a systematic sample of all,

$\% \quad=\quad$ The 'Hart-Becking' spacing index $100 \sqrt{ }\left(\frac{10000}{\mathrm{~N} \times 0.866}\right) / \mathrm{H}_{\mathrm{dom}}$ 
FORM C

EXAMPLE OF RECORD OF REMOVALS FROM A PERMANENT SAMPLE PLOT AND NOTES ON DISEASE PESTS AND ACCIDENTAL DAMAGE

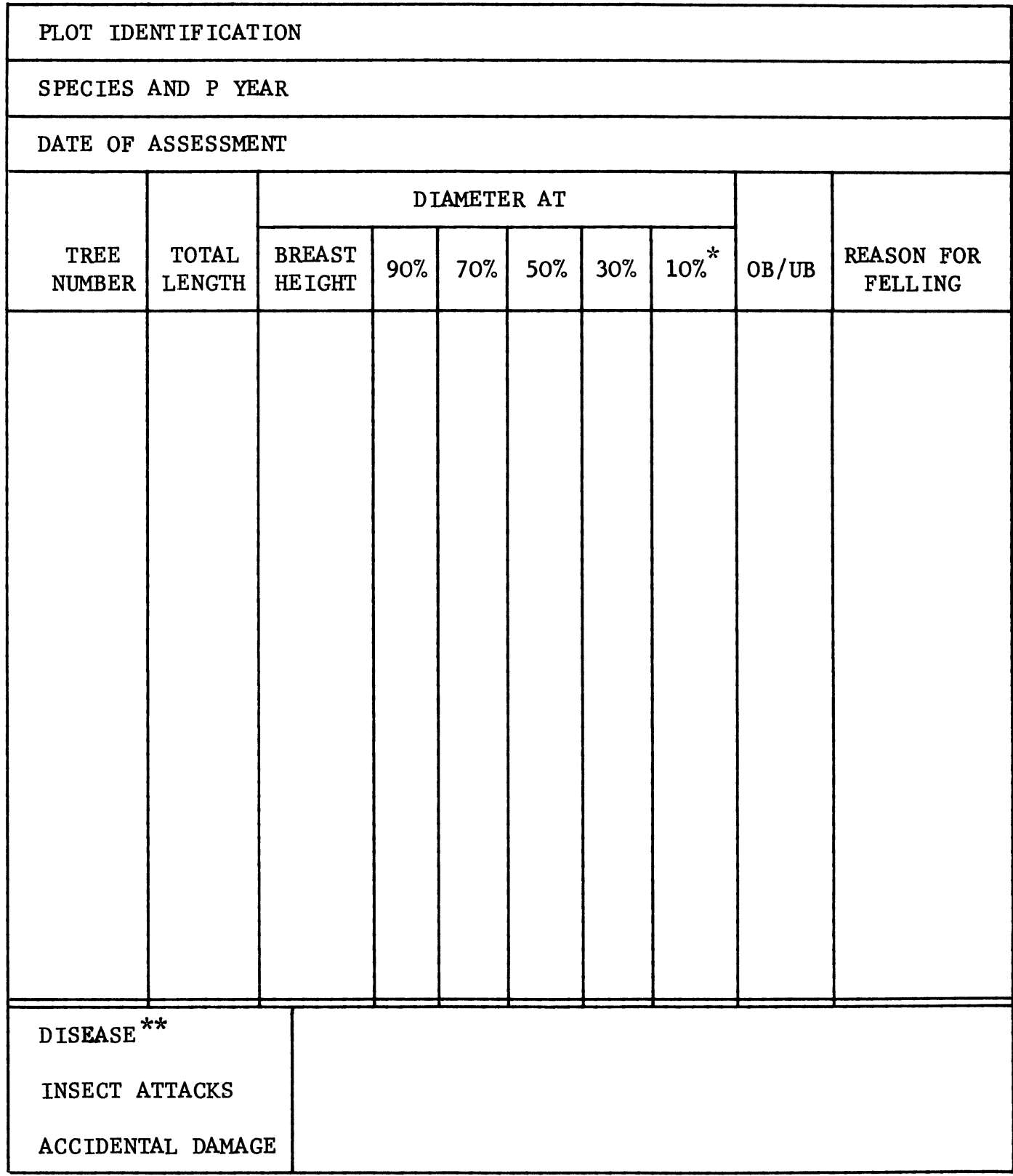

\section{NOTES:}

* Percentages of total length of tree from its top. These may be replaced by fixed lengths of stem.

** If trees have been killed or removed from the plot as a result of disease, pests, windthrow or other damage details will be recorded above whenever possible. Note dates whenever anything unusual occurs. 
Appendix 11. Spacing and stocking tables

(Reproduced from the Commonwealth Forestry Handbook

by kind permission of the Commonwealth Forestry Association)

NUMBER OF PLANTS PER HECTARE FOR VARIOUS SPACINGS IN METRES

\begin{tabular}{|c|c|c|c|c|c|c|c|c|c|c|c|c|c|}
\hline METRES & 1.5 & 2 & 2.5 & 3 & $3 \cdot 5$ & 4 & $4 \cdot 5$ & 5 & 6 & 7 & 8 & 9 & 10 \\
\hline $\begin{array}{l}1 \cdot 5 \\
2 \\
2 \cdot 5 \\
3 \\
3 \cdot 5 \\
4 \\
4 \cdot 5 \\
5 \\
6 \\
7 \\
8 \\
9 \\
10\end{array}$ & $\begin{array}{r}4444 \\
3333 \\
2667 \\
2222 \\
1905 \\
1667 \\
1481 \\
1333 \\
1111 \\
952 \\
833 \\
741 \\
667\end{array}$ & $\begin{array}{r}2500 \\
2000 \\
1667 \\
1429 \\
1250 \\
1111 \\
1000 \\
833 \\
714 \\
625 \\
556 \\
500\end{array}$ & $\begin{array}{r}1600 \\
1333 \\
1143 \\
1000 \\
889 \\
800 \\
667 \\
571 \\
500 \\
444 \\
400\end{array}$ & $\begin{array}{r}1111 \\
952 \\
833 \\
741 \\
667 \\
556 \\
476 \\
417 \\
370 \\
333\end{array}$ & $\begin{array}{l}816 \\
714 \\
635 \\
571 \\
476 \\
408 \\
357 \\
317 \\
286\end{array}$ & $\begin{array}{l}625 \\
556 \\
500 \\
417 \\
357 \\
313 \\
278 \\
250\end{array}$ & $\begin{array}{l}494 \\
444 \\
370 \\
317 \\
278 \\
247 \\
222\end{array}$ & $\begin{array}{l}400 \\
333 \\
286 \\
250 \\
222 \\
200\end{array}$ & $\begin{array}{l}278 \\
238 \\
208 \\
185 \\
167\end{array}$ & $\begin{array}{l}204 \\
179 \\
159 \\
143\end{array}$ & $\begin{array}{l}156 \\
139 \\
125\end{array}$ & $\begin{array}{l}123 \\
111\end{array}$ & 100 \\
\hline
\end{tabular}

NUMBER OF PLANTS PER ACRE AND PER HECTARE FOR VARIOUS SPACINGS IN FEET

\begin{tabular}{|c|c|c|c|c|c|c|c|c|c|c|c|c|}
\hline FEET & 6 & 7 & 8 & 9 & 10 & 12 & 15 & 18 & 20 & 24 & 30 & 33 \\
\hline 6 & $\begin{array}{l}1210 \\
2990\end{array}$ & & & & & & & & & & & \\
\hline \multirow[t]{2}{*}{7} & 1037 & 889 & & & & & & & & & & \\
\hline & 2563 & 2197 & & & & & & & & & & \\
\hline \multirow[t]{2}{*}{8} & 908 & 778 & 681 & & & & & & & & & \\
\hline & 2244 & 1923 & 1683 & & & & & & & & & \\
\hline \multirow[t]{2}{*}{9} & 807 & 691 & 605 & 538 & & & & & & & & \\
\hline & 1994 & 1708 & 1495 & 1329 & & & & & & & & \\
\hline \multirow[t]{2}{*}{10} & 726 & 622 & 545 & 484 & 436 & & & & & & & \\
\hline & 1794 & 1537 & 1347 & 1196 & 1077 & & & & & & & \\
\hline \multirow[t]{2}{*}{12} & 605 & 519 & 454 & 403 & 363 & 303 & & & & & & \\
\hline & 1495 & 1283 & 1122 & 996 & 897 & 749 & & & & & & \\
\hline \multirow[t]{2}{*}{15} & 484 & 415 & 363 & 323 & 296 & 242 & 194 & & & & & \\
\hline & 1196 & 1026 & 897 & 798 & 717 & 598 & 479 & & & & & \\
\hline \multirow[t]{2}{*}{18} & 403 & 346 & 303 & 269 & 242 & 202 & 161 & 134 & & & & \\
\hline & 996 & 855 & 749 & 665 & 598 & 499 & 398 & 331 & & & & \\
\hline \multirow[t]{2}{*}{20} & 363 & 311 & 272 & 242 & 218 & 182 & 145 & 121 & 109 & & & \\
\hline & 897 & 769 & 672 & 598 & 539 & 450 & 358 & 299 & 269 & & & \\
\hline \multirow[t]{2}{*}{24} & 303 & 259 & 227 & 202 & 182 & 151 & 121 & 101 & 91 & 76 & & \\
\hline & 749 & 640 & 561 & 499 & 450 & 373 & 299 & 250 & 225 & 188 & & \\
\hline \multirow[t]{2}{*}{30} & 242 & 207 & 182 & 161 & 145 & 121 & 97 & 81 & 73 & 61 & 48 & \\
\hline & 598 & 512 & $\mathbf{4 5 0}$ & 398 & 358 & 299 & 240 & 200 & 180 & 151 & 119 & \\
\hline \multirow[t]{2}{*}{33} & 220 & 189 & 165 & 147 & 132 & 110 & 88 & 73 & 66 & 55 & 44 & 40 \\
\hline & 544 & 467 & 408 & 363 & 326 & 272 & 217 & 180 & 163 & 136 & 109 & 99 \\
\hline
\end{tabular}


- 225 -

Appendix 11 (cont.)

13. SPACING: SQUARE AND TRIANGLLAR

\begin{tabular}{|c|c|c|c|c|c|}
\hline & & \multicolumn{4}{|c|}{ PLANTS PER UNIT AREA } \\
\hline \multicolumn{2}{|c|}{$\begin{array}{l}\text { UNITS OF } \\
\text { SPACING }\end{array}$} & \multicolumn{2}{|c|}{$\begin{array}{l}\text { SQUARE } \\
\text { SPACING }\end{array}$} & \multicolumn{2}{|c|}{$\begin{array}{l}\text { TRIANGULAR } \\
\text { SPACING }\end{array}$} \\
\hline Feet & Metres & Acre & Hectare & Acre & Hectare \\
\hline 3 & 0.91 & 4840 & 11960 & 5589 & 13810 \\
\hline $3 \cdot 28$ & 1 & 4047 & 10000 & - & 11547 \\
\hline 4 & $1 \cdot 22$ & 2722 & 6727 & $31+4$ & 7768 \\
\hline - & $1 \cdot 50$ & 1798 & 4444 & - & 5132 \\
\hline 5 & $1 \cdot 52$ & 1742 & 4305 & 2012 & 4972 \\
\hline 6 & 1.83 & 1210 & 2990 & 1397 & 3453 \\
\hline - & 2 & 1012 & 2500 & $\bar{x}$ & 2887 \\
\hline 7 & $2 \cdot 13$ & 889 & 2197 & 1026 & 2537 \\
\hline 8 & $2 \cdot 44$ & 681 & 1682 & 786 & 1942 \\
\hline- & $2 \cdot 50$ & 648 & 1600 & - & 1848 \\
\hline 9 & $2 \cdot 74$ & 538 & 1329 & 621 & 1534 \\
\hline- & 3 & 450 & 1111 & $\overline{-1}$ & 1283 \\
\hline 10 & 3.05 & 436 & 1076 & 503 & 1243 \\
\hline- & $3 \cdot 50$ & 330 & 816 & - & 943 \\
\hline 12 & 3.65 & 303 & 747 & 349 & 863 \\
\hline - & 4 & 253 & 625 & - & 72 \\
\hline- & $4 \cdot 50$ & 200 & 494 & - & 570 \\
\hline 15 & 4.57 & 194 & 478 & 224 & 552 \\
\hline & 5 & 162 & 400 & - & 46 \\
\hline 18 & $5 \cdot 48$ & 134 & 332 & 155 & 384 \\
\hline - & $5 \cdot 50$ & 134 & 331 & - & 38 \\
\hline$\overline{-}$ & 6 & 113 & 278 & - & 321 \\
\hline 20 & $6 \cdot 10$ & 109 & 269 & 126 & 311 \\
\hline & 7 & 83 & 204 & - & 236 \\
\hline - & 8 & 63 & 156 & - & 180 \\
\hline - & 9 & 50 & 123 & - & 143 \\
\hline 30 & $9 \cdot 14$ & 48 & 120 & 56 & 138 \\
\hline $32 \cdot 8$ & 10 & 40 & 100 & 47 & 115 \\
\hline \multirow[t]{2}{*}{ ft } & $\mathbf{m}$ & 43560 & 10,000 & $\underline{50298}$ & 11547 \\
\hline & & $\mathrm{ft}^{2}$ & $\mathrm{~m}^{2}$ & $\mathrm{ft}^{2}$ & $\mathrm{~m}^{2}$ \\
\hline
\end{tabular}


Appendix 12. Outline of an experimental plan

$\underline{\text { Title }}$

Heading of the overall programme, or research section Experimental reference number

Title of trial

Year of establishment

Name or title of officer responsible for executing the plan

Description The background to the trial, references to other similar trials in the same programme, reference to overall programme of research.

Objectives Clear statement of objects of the trials, including the type of information expected. The expected duration of the trial and the date for writing up the results should be stated.

Method

1. Location of the trial, details of how to find it, and a location map.

2. The species and provenance involved, with full details of origin.

3. Location of nursery and details of nursery techniques.

4. The design, layout, plot size, size of surrounds, including a site map and plot charts if necessary.

5. Details of any treatments to be superimposed on the basic species/provenance trial.

6. Methods of weeding, thinning, pruning prescribed.

7. Statistical information: degree of accuracy required, and the probability level to be used in testing ( $95 \%$ or $99 \%$ are most usua1).

8. Analysis of variance.

9. Assessments to be made and when.

10. A note on how the results will be analysed.

Resources 1. Details of land requirements, seed requirements and plan requirements from nursery.

2. Specifications of fencing and internal and external demarcation.

3. Organization of machinery if required.

4. Staff requirements, with timetable if possible.

5. Estimates of costs.

Note: The above is a checklist of points to be considered; not all of them may be needed in every trial. 
